Portland State University

PDXScholar

$7-27-2020$

\title{
A Computer Science Academic Vocabulary List
}

David Roesler

Portland State University

Follow this and additional works at: https://pdxscholar.library.pdx.edu/open_access_etds

Part of the Applied Linguistics Commons, and the Computer Sciences Commons Let us know how access to this document benefits you.

\section{Recommended Citation}

Roesler, David, "A Computer Science Academic Vocabulary List" (2020). Dissertations and Theses. Paper 5540.

https://doi.org/10.15760/etd.7414

This Thesis is brought to you for free and open access. It has been accepted for inclusion in Dissertations and Theses by an authorized administrator of PDXScholar. Please contact us if we can make this document more accessible: pdxscholar@pdx.edu. 
A Computer Science Academic Vocabulary List

by

David Roesler

A thesis submitted in partial fulfillment of the requirements for the degree of

Master of Arts

in

Teaching English to Speakers of Other Languages

Thesis Committee:

Lynn Santelmann, Chair

Susan Conrad

Alissa Hartig

Portland State University

2020 


\section{Abstract}

This thesis documents the development the Computer Science Academic Vocabulary List (CSAVL), a pedagogical tool intended for use by English-for-specific-purpose educators and material developers. A 3.5-million-word corpus of academic computer science textbooks and journal articles was developed in order to produce the CSAVL. This study draws on the improved methodologies used in the creation of recent lemmabased word lists such as the Academic Vocabulary List (AVL) (Gardner \& Davies, 2014) and the Medical Academic Vocabulary List (MAVL) (Lei \& Liu, 2016), which take into account the discipline-specific meanings of academic vocabulary. The CSAVL provides specific information for each entry, including part of speech and CS-specific meanings in order to provide users with clues as to how each item is used within the context of academic CS. Based on the comparative analyses performed in this study, the CSAVL was found to be a more efficient tool for reaching an minimal level of academic CS reading comprehension than the widely-used Academic Word List (AWL) (Coxhead, 2000), or the combination of the AWL with the Computer Science Word List (CSWL) (Minshall, 2013). Through coverage tests performed on a variety of corpora, CSAVL was shown to be representative of the written language of academic computer science and focused on the lemmas that are the most relevant to the context of written academic CS. 


\section{Acknowledgements}

I would like to thank Lynn Santelmann, Susan Conrad, Alissa Hartig, and Tanya Sydorenko of the Portland State University Department of Applied Linguistics for their

feedback and support during this project. Their suggestions provided vital guidance in the development of this document. I would also like to thank Errin Beck, Julie Haun, and Alyx Cesar at the Portland State University Intensive English Language Program for providing me with the inspiration for the subject of this thesis. 


\section{Table of Contents}

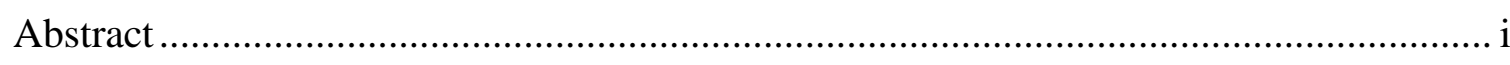

Acknowledgements ................................................................................................ ii

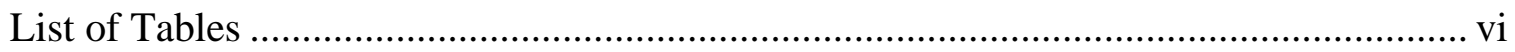

List of Figures ............................................................................................... vii

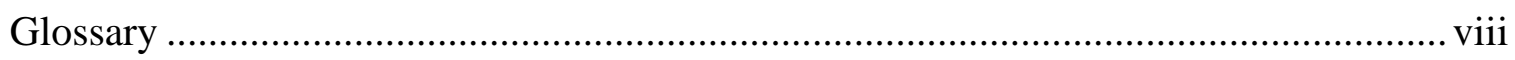

Chapter 1: Introduction....................................................................................... 1

Chapter 2: Literature Review................................................................................. 5

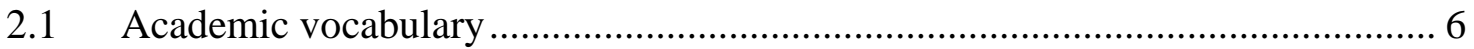

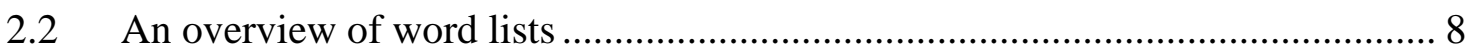

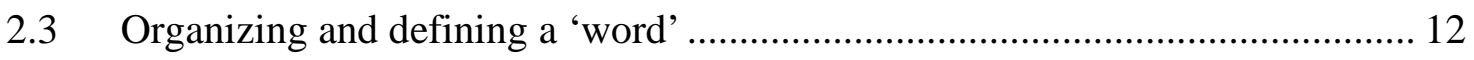

$2.4 \quad$ Lexical coverage and comprehension ............................................................... 15

2.5 Developing a corpus................................................................................... 17

2.6 Creating and evaluating word lists ............................................................... 21

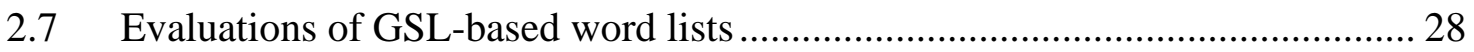

2.8 Evaluations of lemma-based word lists............................................................... 33

2.9 The Computer Science Academic Word List (Minshall, 2013).......................... 37

2.10 Research Purposes of the CSAVL project ......................................................... 40

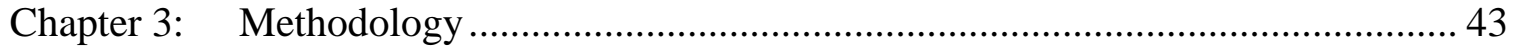

3.1 Corpus design overview .......................................................................... 44

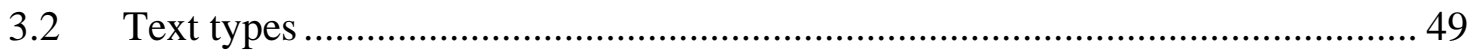

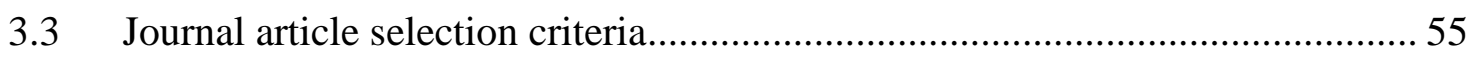

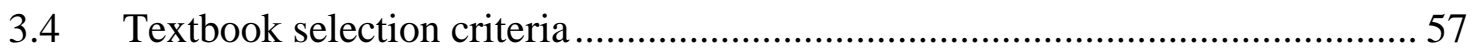

3.5 CSAC1 size and balance .............................................................................. 58

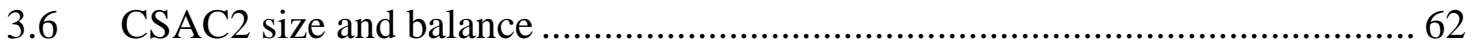

3.7 Data collection \& manual processing............................................................... 66

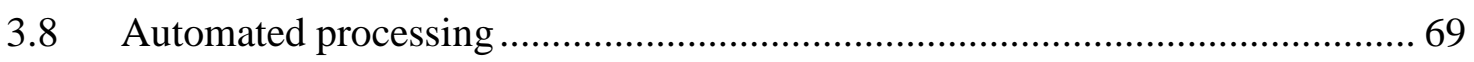

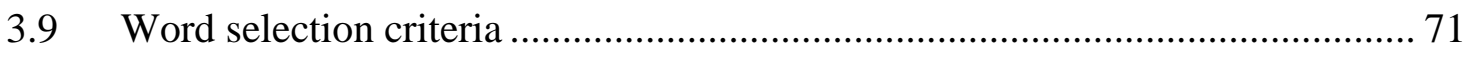


3.10 Tokenization and part of speech tagging................................................... 74

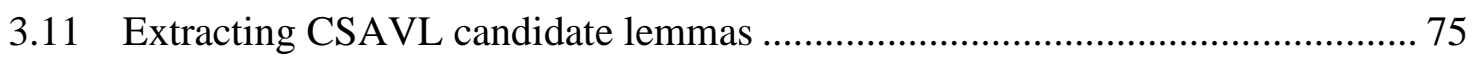

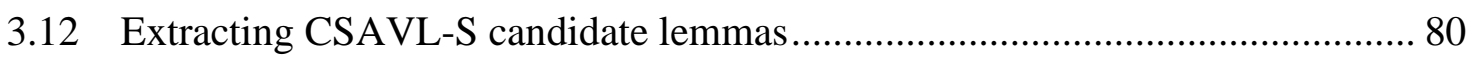

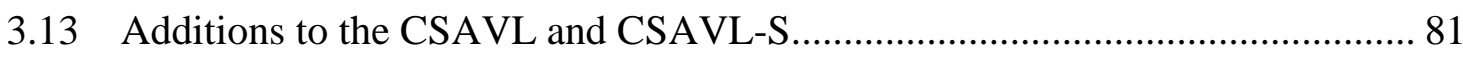

3.14 Removing undesired content from the CSAC2 .......................................... 82

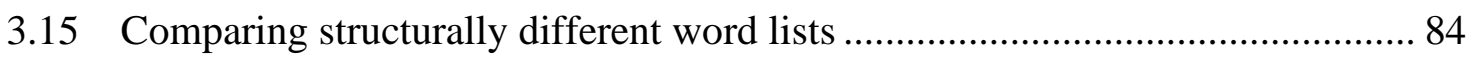

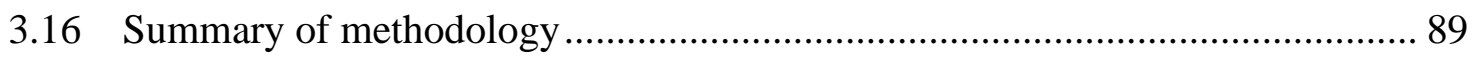

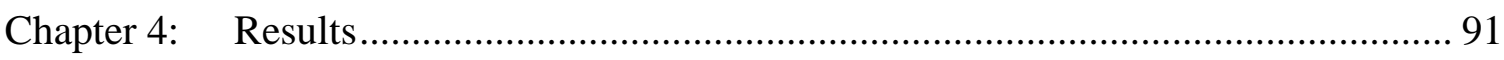

4.1 Academic word list coverage comparisons ................................................. 92

4.2 Supplemental CS word list coverage comparisons ....................................... 97

4.3 General word list coverage comparisons for minimum reading comprehension99

4.4 Coverage across general, academic, and CS corpora................................... 105

4.5 Content comparison with technical dictionaries ......................................... 108

4.6 Word list content comparisons .................................................................. 113

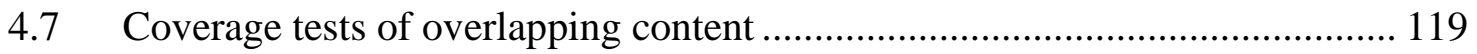

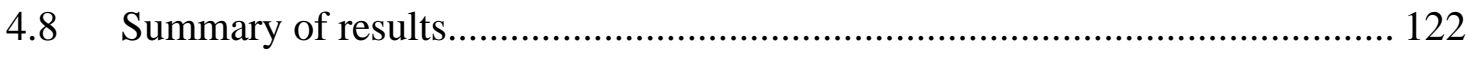

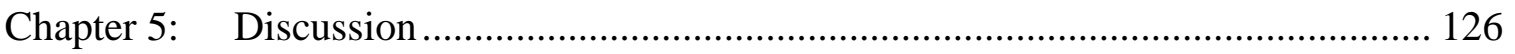

5.1 The coverage and efficiency of the CSAVL and CSAVL-S........................ 127

5.2 Representativeness and technicality of the CSAVL and CSAVL-S ............... 132

5.3 The content of the CSAVL and CSAVL-S ................................................. 134

$5.4 \quad$ Usage of the CSAVL and CSAVL-S ....................................................... 136

5.5 The limitations of the CSAVL project ...................................................... 143

5.6 Possibilities for future corpus and word list research ................................. 145

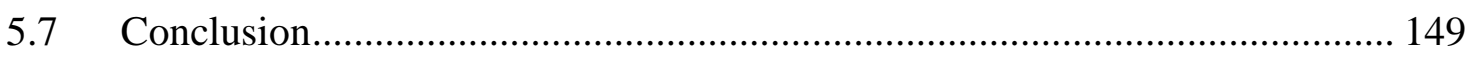

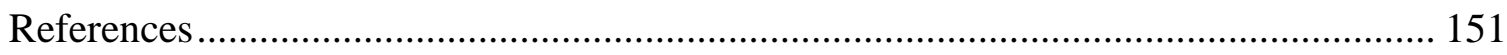

Appendix A: The Computer Science Academic Vocabulary List (CSAVL) ................. 151

Appendix B: The Computer Science Academic Vocabulary Supplemental List (CSAVL-

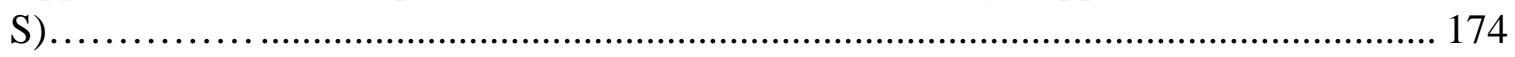

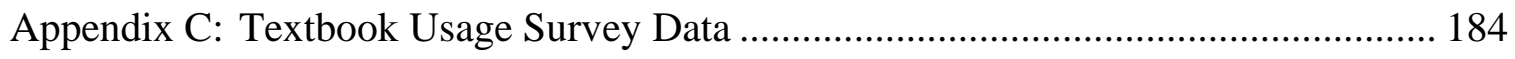

Appendix D: Textbook Usage Counts ..................................................................... 199 
Appendix E: CSAC1 \& CSAC2 Token counts ......................................................... 201

Appendix F: Modified End-of-line Hyphenation Removal Perl Script ........................ 217

Appendix G: ACM Citation Removal Program C++ Code.......................................... 220

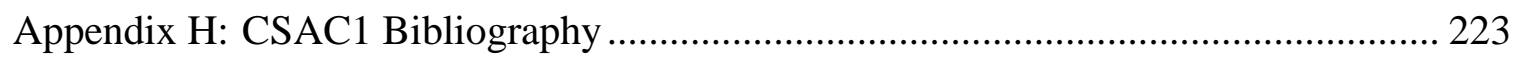

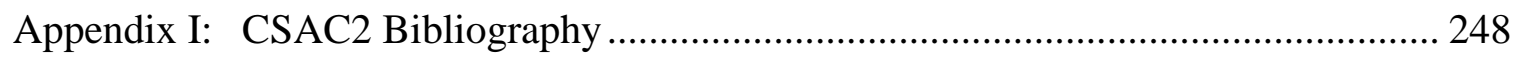




\section{List of Tables}

Table 2.1. Word family levels for “develop” (Bauer \& Nation, 1993) .............................13

Table 2.2 Terms defined here ................................................................................14

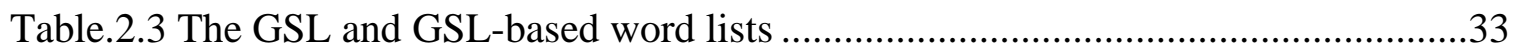

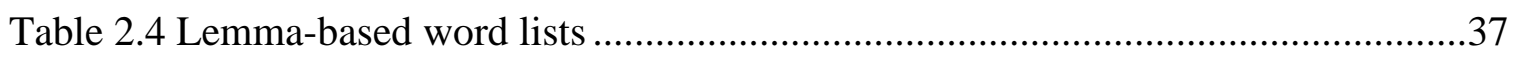

Table 3.1 Planned structure of CSAC1 and CSAC2 2.....................................................46

Table 3.2 Planned sub-corpus structure of CSAC1 and CSAC2 ....................................47

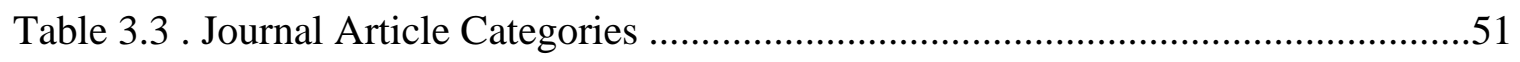

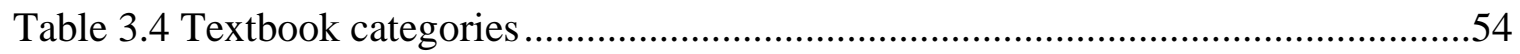

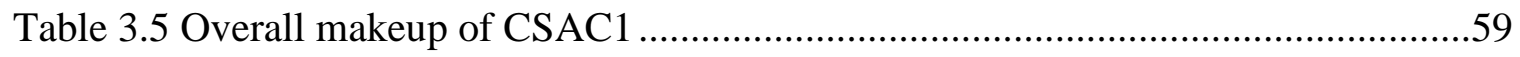

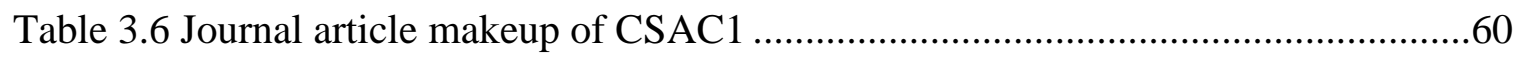

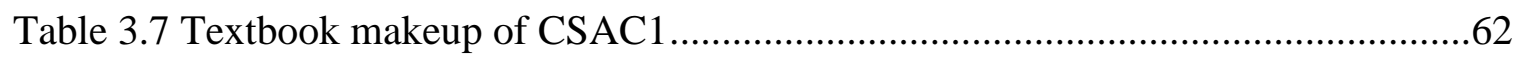

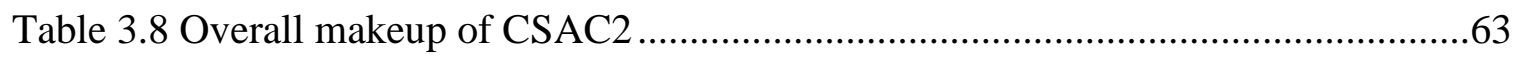

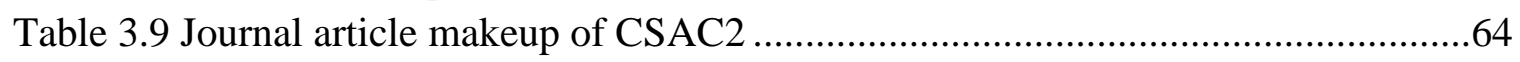

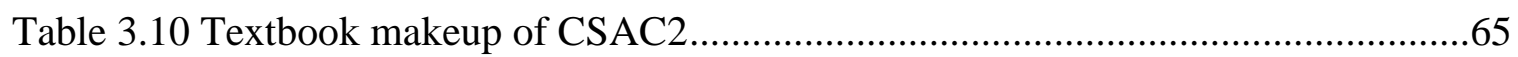

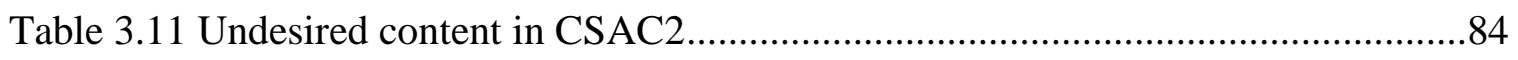

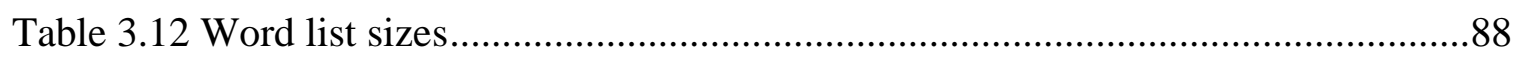

Table 4.1 CSAC2 coverage of the AVL, AWL, and CSAVL .......................................97

Table 4.2 CSAC2 coverage of the AWL, CSWL, CSAVL, and CSAVL-S.....................99

Table 4.3 CSAC2 coverage of general frequency list combinations .............................102

Table 4.4 CSAC2 coverage of NGSL and CSAVL combinations ................................104

Table 4.5 CSAC2 coverage of general, academic, and technical list combinations........105

Table 4.6 Coverage of the CSAVL and CSAVL-S in the BNC academic and non-

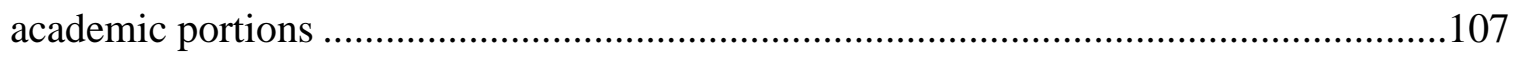

Table 4.7 CSAVL coverage of computer science corpora ...........................................108

Table 4.8 CSAC2 coverage of overlapping and non-overlapping portions of the CSWL

Table 5.1 The 15 most frequent CSAC2 lemmas not covered by the newGSL/CSAVL/CSAVL-S combination..... .131 


\section{List of Figures}

Figure 2.1 An example text with a simulation of $100 \%$, 95\%, and $80 \%$ coverage .......... 15

Figure 2.2 A sampling of the Computer Science Word List (CSWL) (Minshall, 2013).. 40

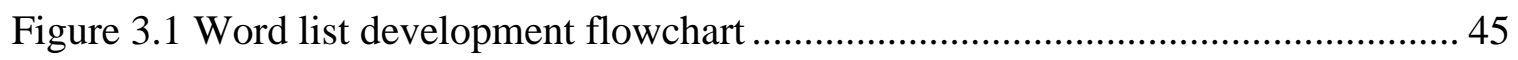

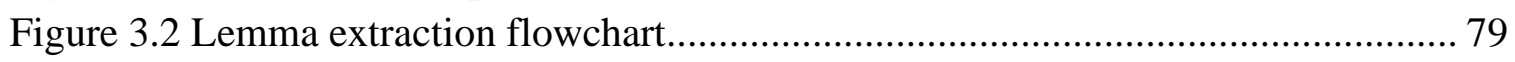

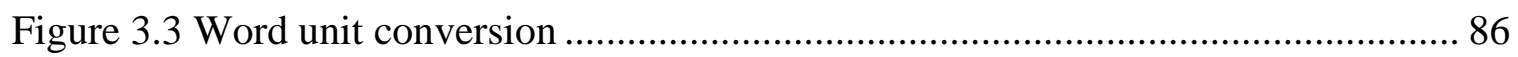

Figure 4.1 Percentages of CSAVL word list lemmas matching technical dictionary entries 111

Figure 4.2 Percentages of CSWL word list lemmas matching technical dictionary entries 113

Figure 4.3 new-GSL lemma overlap with the GSL, AWL, and CSWL ........................ 115

Figure 4.4 CSAVL lemma overlap with the GSL, AWL, and CSWL.......................... 117

Figure 4.5 CSAVL-S lemma overlap with the GSL, AWL, and CSWL ...................... 118 


\section{Glossary}

\begin{tabular}{|c|c|}
\hline Term & Definition \\
\hline ACM & The Association of Computing Machinery \\
\hline AWL & The Academic Word List (Coxhead, 2000) \\
\hline AVL & The Academic Vocabulary List (Gardner \& Davies, 2014) \\
\hline BNC & The British National Corpus \\
\hline CS & Computer science \\
\hline CCS & $\begin{array}{l}\text { The Computing Classification System (used by the ACM to } \\
\text { categorize texts) }\end{array}$ \\
\hline COCA & The Corpus of Contemporary American English (Davies, 2008) \\
\hline CSWL & The Computer Science Word List (Minshall, 2013) \\
\hline CSAC1 & $\begin{array}{l}\text { The Computer Science Academic Corpus } 1 \text { (used to generate the } \\
\text { CSAVL) }\end{array}$ \\
\hline CSAC2 & $\begin{array}{l}\text { The Computer Science Academic Test Corpus } 2 \text { (used to evaluate } \\
\text { the CSAVL) }\end{array}$ \\
\hline CSAVL & The Computer Science Vocabulary List (the subject of this thesis) \\
\hline CSAVL-S & The supplemental list to the Computer Science Vocabulary List \\
\hline DDL & Data-driven learning \\
\hline DII & The Download Immediacy Index (Wan et al., 2010) \\
\hline ELL & English language learner \\
\hline ESP & English for specific purposes \\
\hline ESL & English as a second language \\
\hline EAP & English for academic purposes \\
\hline GSL & The General Service List (West, 1953) \\
\hline IEEE & The Institute of Electrical and Electronics Engineers \\
\hline lemma & Base word, all inflections, single part of speech \\
\hline
\end{tabular}


MAVL The Medical Academic Vocabulary List (Lei \& Liu, 2016)

MAWL The Medical Academic Word List (Wang et al., 2008)

modified lemma Base word, all inflections, part of speech is ignored (Browne, 2014)

new-GSL The New General Service List (Brezina \& Gablasova, 2015)

NGSL The New General Service List (Browne, 2014)

ODOCS The Oxford Dictionary of Computer Science (Butterfield et al., 2016)

ODOM The Concise Oxford Dictionary of Mathematics (Clapham \& Nicholson, 2009)

POS Part of speech

PSU Portland State University

token Word unit used in running counts

type Word unit used to count different forms

UWL The University Word List (Xue \& Nation, 1984)

word family Headword, all inflections, and all derivations (up to level 6), includes homographs 


\section{Chapter 1: Introduction}

In the United States, international students make up a large number of the students enrolled in science and engineering-related disciplines. According to data from the National Foundation for American Policy (2017), international students make up the majority of students enrolled in computer science graduate programs at about 90 percent of American universities. Of these students, many will likely be enrolled in English for Academic Purposes (EAP) programs at their respective institutions, where they will receive ESL instruction in order to prepare them for study in their intended academic discipline. In many university EAP programs, students bound for a wide variety of specific academic disciplines are grouped into courses which are designed around the goal of providing general academic English instruction and can incorporate a one-sizefits-all approach to course content. In order to provide instruction that can meet the needs of the varied goals of students headed for a multitude of disciplines, a set of general academic vocabulary, such as that described by the Academic Word List (AWL) (Coxhead, 2000), is often adopted as a guiding element in curriculum design and course planning.

Word lists such as the AWL attempt to represent academic vocabulary as a whole by identifying frequently used words within corpora of texts drawn from a variety of academic disciplines. Word lists can allow limited resources to be efficiently allocated in course design by focusing learners' attention on the vocabulary that will likely be most 
frequently encountered in academic reading. However, an approach that relies on a general academic vocabulary ignores significant differences in meaning and usage of words as they occur in various disciplines (Hyland \& Tse, 2007; Lam, 2001). This can result in students devoting resources to studying words meanings that may never be encountered in their own discipline, or are used in completely different ways in that discipline. In the discipline of computer science, words such as "bug”, “port”, “tree”, “string”, and “mouse” take on discipline-specific meanings that substantially differ from their general-usage meanings. By only focusing on the general-usage meanings of these context-sensitive words, learners have been found to face additional obstacles when encountering them situated in discipline-specific texts (Lam, 2001). The lack of contextspecificity in general and academic lists such as the GSL and AWL thus limits the claims of efficiency that lead educators to adopt the use of these word lists. Additionally, the efficiency of general academic word lists is limited by the form of how words are defined and organized within these lists. Word lists (like the AWL) that do not differentiate words by parts of speech can prompt learners to study words such as handle in both verb and noun forms, when only one of those usages might be frequent in the learner's intended discipline.

At Portland State University (PSU), where this thesis was written, a “ $2+2$ ” program is offered to international students studying computer science (CS). Students who are enrolled in this program study CS for two years in Changchun, China, and then finish the third and fourth year of their degrees in the United States at PSU. Before arriving in the US, these students receive English as a Second Language (ESL) training that was 
developed by the faculty of PSU's Intensive English Language Program (IELP). At the request of the PSU's IELP, which seeks to develop English for specific purposes (ESP) materials specifically for computer science students, this thesis project was undertaken in order to provide a corpus and word list that can better meet the needs of a group of learners that is preparing for ESL study in the context of academic computer science. In order to achieve the most efficient route to academic computer science reading comprehension for these learners, the inclusion and identification of words with CS discipline-specific meanings is an important step to mitigate issues that may arise from the transfer of general-usage word meanings into CS-specific contexts.

This study attempts to address some of the issues observed in previously-created word lists through the creation of the Computer Science Academic Vocabulary List (CSAVL) (located in Appendix A) and the Computer Science Academic Vocabulary Supplemental List (CSAVL-S) (located in Appendix B), a pair of discipline-specific lists that are informed by the improved methodologies used in the creation of other recent ESP word lists, take advantage of technological advances, and are representative of the written language of the discipline while remaining focused on user efficiency.

This thesis is divided into a total of five chapters. This introductory chapter is followed by Chapter 2, which contains a literature review and a statement of the purposes for this project. Chapter 3 describes the methodology employed in creating the Computer Science Academic Corpus 1 and 2 (CSAC1 and CSAC2) and the Computer Science Academic Vocabulary List (CSAVL). Chapter 4 reports the results of analyses of the 
CSAVL word list, and Chapter 5 concludes the document with a discussion of the results and their possible implications for word list users. 


\section{Chapter 2: Literature Review}

The literature review chapter begins with a review of research on the topic of academic vocabulary, and is followed in Section 2.2 with an overview of existing word lists and their purposes. Section 2.3 contains a summary of the systems which have been used to organize and define a "word" in previous studies. This is followed by a review of research regarding the relationship between lexical coverage and reading comprehension in Section 2.4. The following two sections (2.5 and 2.6) review the considerations that should be made during corpus and word list creation as well as the methods for evaluating word lists. Research which has made evaluations of word lists based on West's General Service List (GSL) (1953) is reviewed in Section 2.7, and research which has evaluated word lists that are organized around the lemma structure appears in Section 2.8. A review of the choices made in the development of a word list based on a similar topic, the Computer Science Word List (CSWL) (Minshall, 2013), are reviewed in Section 2.9, and the chapter concludes with a statement of the purposes of this project in Section 2.10 . 


\subsection{Academic vocabulary}

Academic vocabulary knowledge has been described as being vital to both reading comprehension (Corson, 1997; Jacobs, 2008; Nagy \& Townsend, 2012) and academic success (Goldenberg, 2008). Knowledge of academic vocabulary has also been used to gatekeep access to educational systems through standardized tests such as the TOEFL, GRE, and SAT. As noted by Maylath (1996, 2013), the verbal components of these preuniversity standardized exams can focus heavily on the Graeco-Latin vocabulary of English, a subset of English vocabulary which is removed from the everyday language practices of most speakers of English. Additionally, researchers have found items of academic vocabulary to often be of greater morphological complexity than words common to everyday speech (Nagy \& Anderson, 1984; Nippold, 2007). These two factors (Graeco-Latin origins and morphological complexity) can increase the learning burden for learners of academic vocabulary whose known languages do not share morphological characteristics with English or have little to no Graeco-Latin lexical overlap (Hsia et al., 1995) and increases the need for instruction that focuses specifically on the formalized register of academic English.

The demand for academic English instruction has led to the development of many English for Academic Purposes (EAP) programs in order to prepare learners for the comprehension of the specialized vocabulary and the production of the formal structures that are specific to this variety of English. To address the planning, development, and training needs of academic English instruction focused on vocabulary acquisition, 
numerous authors have produced works detailing the characteristics of academic vocabulary and have proposed approaches to academic vocabulary instruction (Bauman \& Graves, 2010; Beck et al., 2002; Biemiller, 2010; Gardner, 2013; Graves, 2006; Carter, 2012; Nagy \& Townsend, 2012; Nation, 2001, 2008; Zimmerman, 2009). These authors have called for a context-focused approach to vocabulary that encourages both educators and learners to maintain an awareness of how vocabulary items are situated in relation to the topic, the writer, and the known vocabulary within the text. These authors have also called for the explicit instruction of academic vocabulary and have argued for the building of academic vocabulary lists that are discipline- or domain-specific (e.g. academic medical vocabulary or academic engineering vocabulary).

Although context-focused instructional methods and explicit instructional methods might be interpreted as being in opposition to one another, Nation (2001) states that these two methods are instead complementary activities. Research has provided evidence that combinations of context-based and explicit methods, such as presenting words to learners before reading (Jenkins et al.,1984) and defining words that are situated in context (Elley, 1989), increases the amount of vocabulary learning. These forms of academic vocabulary instruction, which are both explicit and context-situated, can be facilitated through the use of discipline-specific academic vocabulary lists. These word lists, which represent the usage of vocabulary in a specific disciplinary context, can complement the reading of authentic texts drawn from that discipline and can be used to prioritize the study of the academic vocabulary items that are most frequently used within that discipline. 


\subsection{An overview of word lists}

A common tool for the study of academic vocabulary is the word list. A word list is typically a list of the most frequently occurring items in a collection of texts (a corpus). Word lists can be useful tools for both the study of specialized vocabulary and for pedagogical applications such as course, assessment, and materials design. Nation (2016) states that there is a cost/benefit principal for language learners which "says that learners should get the best return for their learning effort” (p.5). Following this principle, the most frequently used words in a language should be the first vocabulary focus of a language course. A knowledge of the most frequent words will allow learners to recognize a large portion of the terms included in a text (the 100 most frequent words of English can cover $50 \%$ of a given text), and also begin to make inferences about the meanings of unknown vocabulary items based on their context and use in relationship to the known items. Software tools such as those found on the Compleat Lexical Tutor website (Cobb, n.d.) can allow educators to use high-frequency word lists to identify the words in a text that are likely to be unfamiliar to language learners in order to prepare lessons that focus on these items or to gauge the appropriateness of a text for learners of a known proficiency level.

In a classification of vocabulary types, Nation (2013) distinguished three bands of vocabulary based upon frequency: high-frequency words, mid-frequency words, and lowfrequency words. High-frequency words include all function words such as “the”, “a”, and "in” (for the case of English) and also many content words such as "pleasant”, 
“question”, and “pull”. These items can make up as much as $90 \%$ of a text and are the most likely items to be encountered in general-usage written contexts by language learners. Mid-frequency words (“nod”, “pupil”, “evolution”) cover roughly 9\% of a text. Low-frequency words (“bifurcate”, “eponymous”) cover about 1\% of a text, and include proper nouns and words that may appear only a single time within a text.

In addition to these three frequency bands, Nation also described two types of specialized vocabulary: academic words and technical words. Academic words are defined as those words that typify the register of general academic language and can be found in a variety of academic texts across disciplinary boundaries. The third category, technical words, are those that are closely related to a specific topic area or discipline. These words are found frequently in the topic they are associated with and may be found less frequently outside of that topic. Academic words can make up $9 \%$ of the words in a text, and technical words can make up 20 to 30\%. Words in the academic and technical categories may be found within all three of the frequency bands. A word such as "memory”, which has a discipline-specific meaning in computer science (CS) and a nontechnical meaning outside of CS, could be considered an item of technical vocabulary that could be found in the high-frequency word band.

Word lists that are concerned with describing the most highly frequent lexical items in a general usage context will be referred to here as general word lists. The most wellknown of the English general word lists is The General Service List (GSL) (West, 1953). The GSL, one of the most widely used word lists in English language education, represents the 2000 most frequently used words in general English. The GSL was 
designed with the purpose of selecting a core vocabulary for English as a foreign language instruction (Gilner, 2011) and was created using a combination of objective criteria (word frequency and range in a collection of texts) and subjective criteria (difficulty of learning, necessity, style). The word frequency counts were manually performed in order to create the GSL, and it was not until the late 1960's that computer tools and digital corpora (collections of texts) such as the one-million-word Brown Corpus of American English (Francis \& Kučera, 1967) began to appear.

Lists of academic vocabulary are created in order to aid educators in setting vocabulary goals, selecting texts for instruction, creating learning materials, and designing and planning English for Academic Purposes (EAP) programs. Academic vocabulary lists can also provide guidance for independent learners seeking an efficient path to developing the vocabulary knowledge necessary for reading comprehension (Hu \& Nation, 2000; Laufer, 1989; Laufer \& Ravenhorst-Kalovski, 2010). Academic vocabulary can cause difficulties for learners since these words often appear less frequently than general vocabulary items and can also take on special meanings in the context of an academic discipline (Cohen et al., 1979; Lam, 2001).

A number of academic vocabulary lists were developed in the 1970s, with some using corpus techniques (Campion \& Elley, 1971; Praninskas, 1972) and others making manual counts of textbook annotations made by learners (Ghadessy, 1979; Lynn, 1973). These lists were later combined by Xue and Nation (1984) to create their University Word List (UWL). The UWL was used by learners, teachers, course designers, researchers until a replacement list, the Academic Word List (AWL), was published in 
Coxhead (2000). The AWL was corpus-based and had the defined purpose of aiding students intending to read academic texts while studying at the university level in an English-speaking country. The AWL was intended to serve the purposes of university students from a wide variety of subject majors, and the corpus used to derive the AWL was composed of a careful selection of texts from a variety of academic disciplines. Coxhead's AWL excluded the content of the General Service List (West, 1953), and was designed to be used by learners who had already developed familiarity with the contents of the GSL. The AWL has been widely adopted due to its efficiency and utility in EAP contexts (Coxhead, 2016).

In recent years, researchers have continued to use new methodologies to develop new word lists that either serve as replacements to the GSL (Brezina \& Gablasova, 2015; Browne, 2014) or the AWL (Gardner \& Davies, 2014), or have instead focused on creating lists that describe the academic vocabulary of a specific discipline such as business (Hsu, 2011), medicine (Hsu, 2013; Lei \& Liu, 2016; Quero, 2015; Wang et al., 2008), Science (Coxhead \& Hirsch, 2007), agriculture (Martinez et al., 2009), applied linguistics (Khani \& Tazik, 2013; Vongpumivitch et al., 2009), engineering (Hsu, 2014; Ward, 2009), nursing (Yang, 2015), pharmacology (Fraser, 2007), and computer science (Minshall, 2013). The subject of this thesis, The Computer Science Academic Vocabulary List (CSAVL), continues in this trend of new word lists that are increasingly focused on specific academic disciplines. The CSAVL, however, incorporates an improved methodology that allows it to describe the vocabulary of academic computer science in a more detailed way than any previously-created word list. 
The following sections of this chapter provide additional background information on the considerations and processes that are involved in the development of a word list, beginning with the definition of "word" as an organizational unit.

\subsection{Organizing and defining a 'word'}

In order to understand the guiding principles that lead to the creation of word lists, it is first necessary to examine how different researchers have chosen to define and group words in the past. When counting the number of words within a text, words are often counted in types and tokens. Tokens are used for running word counts, while types are a count of the number of different words in a text. In "The dog sees the cat", there are five tokens, but only four types (the appears twice). Whereas the terms type and token are primarily used for counting, other terms such as lemma, lempos, and word family are used to organize words into groups. In word lists such as the AWL, words are grouped into word families (Bauer \& Nation, 1993). Bauer and Nation developed the word family as an organizational system that classifies related words into a six-level hierarchy that ranks affixes based on productivity, predictability, and regularity. A word family is centered on a headword, and includes all inflected forms of that headword along with all derivations up to level six of Bauer and Nation’s (1993) ranking system. Table 2.1 illustrates the ranking system applied to the headword develop. 
Table 2.1. Word family levels for “develop” (Bauer \& Nation, 1993)

\begin{tabular}{ll}
\hline $\mathbf{2}$ & develop (headword), develops, developed, developing \\
$\mathbf{3}$ & developable, undevelopable, developer(s), underdeveloped \\
$\mathbf{4}$ & development(s), developmental, developmentally \\
$\mathbf{5}$ & developmentwise, semideveloped, antidevelopment \\
$\mathbf{6}$ & redevelop, predevelopment \\
\hline
\end{tabular}

According to Bauer and Nation, "the levels can be viewed as an attempt to set up levels of word transparency”. In their view, learners who have learned rules for inflection will be able to recognize the relationship between all inflected forms (level two). Levels 3-6 include derivational forms that are ranked by their degree of transparency. Lists based on word families make the assumption that if learners recognize a headword, they will also have knowledge of the derivational forms up to level 6 (i.e. learners who recognize develop will also be assumed to be able to infer the meaning of antidevelopment). Although Bauer and Nation did not intend to include homographs within word families, due to limitations of their software in recognizing parts of speech, words like bar (verb) and bar (noun) are grouped into the same word family in word family-based lists.

Additional issues that arise from word lists based around word families are discussed in detail in Section 2.7.

The term lemma is used here to refer to all inflectional forms of a word (the equivalent of level 2 in the word family rankings) and only includes one part of speech (if kick as a noun is included, the verb is not). A lemma can be flagged with its part of speech in order to indicate which forms are included. The lemma “develop_v” would 
include the forms develop, develops, developed, and developing (all inflected verb forms). In a noun phrase such as "the developing industry”, where “developing” is a present participle modifying a noun, it would be classified as an adjective “developing_adj”. This would be a separate lemma from the present continuous use (as in “we are developing a solution”), which would be included in “develop_v”. There is overlap of the forms (spellings) that are grouped in different lemmas (the form “developing” can be found in both the “develop_v” and “developing_adj” lemmas), and in order to classify forms accurately, the adjacent words in the context that they are found must be examined to determine the part of speech. Without the contextual clues provided by adjacent words, it is not possible to accurately determine whether “developing” belongs to “develop_v” or “developing_adj”. For the reader’s reference, the terms defined in this section are listed in Table 2.2. A more detailed discussion of the differences between the lemma and the word family appears in Section 3.14.

Table 2.2 Terms defined here

\begin{tabular}{ll}
\hline token & used in running counts \\
type & used to count different forms \\
word & headword, all inflections, and all derivations (up to level 6), includes \\
family & homographs \\
lemma & base word, all inflections, single part of speech \\
\hline
\end{tabular}




\subsection{Lexical coverage and comprehension}

When evaluating the representativeness of a word list, the term lexical coverage is used to describe the percentage of tokens in a given corpus that correspond with items on the word list. Figure 2.1 provides a simulation of the reading experience for a learner who comprehends $100 \%$ of a text (top image), $95 \%$ of text (center image), or $80 \%$ of a text (bottom image). In an ideal scenario, a word list would be able to provide $100 \%$ coverage of a text, and after studying the contents of that list, a learner would ideally recognize all of the items in the text. However, in practice, word lists usually provide less than full coverage of a text.

When you declare an ordinary variable $\mathrm{x}$ to have the data type int, the $\mathrm{C}++$ compiler allocates a memory cell that can hold an integer. You use the identifier $x$ to refer to this cell. To put the value 5 in the cell, you could write

int $x=5$;

When you an ordinary variable $\mathrm{x}$ to have the data type int, the $\mathrm{C}++$ compiler memory cell that can hold an integer. You use the identifier $x$ to refer to this cell. To put the value 5 in the cell, you could write

int $x=5$;

When you $\quad$ an ordinary $\quad$ type int, the $\mathrm{C}++$ compiler
the cell, you could write
int $\mathrm{x}=5$;

Figure 2.1 An example text with a simulation of coverage of 100\% (top box), 95\% (middle box), and 80\% (bottom box) 
Lists such as the General Service List (GSL) (West, 1953) have been found to cover between $78 \%$ and $92 \%$ of various corpora, with an average of around $82 \%$ coverage (Nation \& Waring, 1997). The lexical threshold is described by Laufer (1989) and Laufer and Ravenhorst-Kalovski (2010) as the amount of known vocabulary required for an “adequate” level of reading comprehension. In Laufer (1989), 95\% lexical threshold represented the portion of vocabulary accurately recognized by those students who achieved the minimum passing grade (55\%) on a reading comprehension examination at the University of Haifa. Laufer found that at 95\% lexical coverage there were significantly more participants with a passing reading comprehension exam score. In a later study, Hu and Nation (2000) found that 90-95\% lexical coverage allowed students to perform minimally, and 98\% was suggested to be a more appropriate threshold for an adequate level of comprehension (where “adequate” is defined as a score of $85 \%$ or higher on a written recall test). In Nation (2001), it was stated that Laufer’s 95\% threshold was likely to be a "standard of minimally acceptable comprehension" (p.147). Laufer and Ravenhorst-Kalovski (2010), revisiting the topic of the lexical threshold twenty years later, made a further clarification: the 95\% threshold was determined to be “adequate” if readers are provided with "some help and guidance” (p.25), and the 98\% threshold was determined to be "adequate" in the sense of a learner having the ability to read independently and pass an Israeli EFL study exemption exam (part of a university entrance test).

For the present project (the development of the Computer Science Academic Vocabulary List), the lexical threshold of 95\% will follow Nation’s (2001) description as 
being the amount of lexical coverage necessary for a minimal level of reading comprehension. Given that a learner who knows the 2000 headwords of the GSL will have an average lexical coverage of $82 \%$ of basic texts, and based on the findings of the lexical threshold studies described above, that learner will requires an additional 13\% lexical coverage in order to reach a minimal level of reading comprehension.

\subsection{Developing a corpus}

An initial stage of word list creation is the selection or creation of a corpus, or body of texts, that information about word frequency and distribution can be drawn from. When evaluating an existing corpus, or designing a new one for the development of a word list, the necessary considerations include representativeness of the target variety (Biber, 1993; Clancy, 2010), corpus size (Biber, 1993; Reppen, 2010; Sinclair, 1991), and the organization and balance of text types within the corpus (Nation \& Sorell, 2016; Sinclair, 2005; Sorell, 2013).

\section{Representativeness}

Linguistic features such as vocabulary vary from one register to the next (Biber, 1989) and the meanings of lexical items can also vary across registers (Hyland \& Tse, 2007). A corpus that is created to describe the vocabulary of a specialized academic discipline should be composed of text that are representative of that discipline. Biber 
(1993) stated that representativeness "refers to the extent to which a sample includes the full range of variability in a population” (p. 243). In order to represent the full range of lexical choices made in a written register such as academic computer science, the selected texts for the corpus should be typical of the register, but at the same time should also be varied enough to include a wide range of text types and topics. For the case of the Computer Science Academic Vocabulary List (CSAVL), texts should be clear examples of academic computer science writing, and include as many text types and subdisciplinary topics as possible (such as programming, hardware, and networking). In addition to including a variety of types and topics, including short texts by a variety of authors can maximize the variety of lexical items within the corpus (Sutarsyah et al., 1994) and minimize the author bias that can result from the idiosyncratic style of a single writer (Atkins et al., 1992; Sinclair, 1991).

When discussing representativeness, Biber (1993) also pointed out the importance of defining the target population of texts. For a corpus that will be used to create an academic word list, the identification of the target population of texts should be informed by the situational characteristics of the educators and learners who will be the users of the word list. Factors relevant to choosing text types for corpus and word list creation are described in Nation and Sorell (2016) as being geographical location, age, and learning situation. Varieties of English found in the US or UK feature lexical differences that should also be a consideration when developing a corpus or word list. The users of the CSAVL will be students studying at American universities and in order to reflect this context, the target text population can be defined as texts currently utilized by computer 
science programs at American universities. Regarding age and learning situation, users of the CSAVL will be adult students in English for Academic Purposes courses who have the intention of studying computer science at an American university. Considering these factors, the types of the target population of texts might include CS textbooks, academic journal articles, or course materials.

\section{Corpus size}

As noted in Reppen (2010), there is no one-size-fits-all solution to the question of corpus size; the size of a corpus should be based upon the intended purpose for that corpus. Biber (1990) argued that a corpus of one million tokens (such as the Brown corpus) is an adequate size for a lexical study attempting to identify rare words, provided that the corpus contains texts that are representative of the full range of the language variety. For the development of an academic word list that focuses the most frequent terms rather than identifying rare words, the minimum corpus size may be smaller (Sinclair, 2005). For the purposes of creating academic word lists, most corpus sizes have varied from 1 million to 6.2 million words (Coxhead, 2000; Khani \& Tazik, 2013; Lei \& Liu, 2016; Wang et al., 2008), whereas one of the largest corpora used for an academic list has been the 120-million-word corpus used to create Gardner and Davies’ (2014) Academic Vocabulary List. For the Computer Science Academic Vocabulary List, a corpus size of 3.5 million words will be used, following the examples of the similarlypurposed Academic Word List (Coxhead, 2000) and the Computer Science Word List (Minshall, 2013). 


\section{Organization and balance}

Corpus organization is also tied to the idea of representativeness, with Biber (1993) arguing that texts should have a hierarchical organization where text categories are clearly defined based on those observed in the target population of texts. For corpora representing a register such as academic English, text categories have been formed based on general academic disciplines such as “Science”, “Arts”, and “Law” (Coxhead, 2000; Gardner \& Davies, 2014). A subsection of a corpus (such as “Arts”) is referred to as a sub-corpus. Forming sub-corpora within the main corpus allows for the use of range and dispersion measures: range being a measure of how many sub-corpora an item appears in (this could tell us that a word appears in “Arts" and "Law”, but not "Science”); and dispersion being a measure of word distribution throughout the corpus (which might tell us that appearances of a word such as "legal" are more clustered in the "Law" sub-corpus than in other areas).

The division of a corpus into sub-corpora should be done with an attention to balance. Nation and Sorell (2016) argue that each sub-corpus should be of equal size; and each sub-corpus should be made up of similar types of texts. If sub-corpora are not of equal size, the range measurement may become distorted: a large sub-corpus will provide more opportunities for low-frequency words to appear, while a small sub-corpus will provide fewer. Additionally, a sub-corpus composed of differing text types may produce unpredictable results when range is measured since different types of academic texts (e.g. textbooks and journal articles) have been found to contain variation in their linguistic 
features (Conrad, 1996) or could represent different modalities (transcriptions of spoken interactions versus written texts).

Following the arguments made regarding corpus organization and balance in Biber (1993) and Nation and Sorell (2016), the 3.5-million-word corpus that will be used to develop the Computer Science Academic Vocabulary List will be divided into equally sized sub-corpora based upon sub-disciplinary categories of academic computer science (such as "Hardware” or "Programming”) and further divided by text type (textbook or journal article).

\subsection{Creating and evaluating word lists}

After choosing or designing a source corpus that is representative, appropriatelysized, and balanced and that reflects the intended purpose of the word list, several critical considerations should be addressed. The first of these considerations is the form of counting and organizational structure that will define a "word" in the list. For academic vocabulary lists such as the Academic Word List (Coxhead, 2000) or the Academic Vocabulary List (Gardner \& Davies, 2014), this has been either the word family or lemma (described in Section 2.3). The second considering is the word selection criteria (such as minimum frequency) which will be used to extract the contents of the word list from the source corpus. The third consideration is the method of list evaluation, which 
will be used to determine the degree to which a list might successfully meet the needs of its intended users.

\section{The unit of counting}

Word lists of general and academic vocabulary have primarily counted words using either the word family (Coxhead, 2000; Wang et. al, 2008; Xue \& Nation, 1984) or the lemma (Gardner \& Davies, 2014; Lei \& Liu, 2016). Since the word family unit includes all parts of speech, all inflectional forms, and most derivations forms (as described in Section 2.3), word lists based on the word family may appear to be "shorter" in length. Nation (2016) suggests there is an approximate ratio of 1:3:6 when converting between word families, lemmas, and types. A single word family (“walk”) may convert to approximately three lemmas (“walk_v”, “walk_n”, “walking_n”, “walking_adj”, “walker_n”) and six types (“walk”, “walks”, “walked”, “walking”, “walker”, “walkers”). When comparing lists based on different units of counting, this proportional conversion relationship should be considered (a detailed discussion of this kind of comparison provided in Section 3.15).

While word families are fairly inclusive groupings of inflections and derivations, the lemma is a more specific unit, and lemma-based lists can provide a more precise description of the frequent items in a corpus (Brezina \& Gablasova, 2015). A word family-based word list (which ignores part of speech) developed using a corpus that contains many instances of "abstract” as an adjective and no instances as a noun, would give list users no indication of this difference. As noted by Gardner (2007), word family- 
based lists also assume that derivational forms are transparent to learners and that learner familiar with items such as "please” will also understanding the meaning of derivational forms such as “unpleasant”. Though lemma-based lists can provide greater specificity in their representation of a register that can result in a heightened level of guidance for list users, when developing a lemma-based word list, additional challenges may be faced by word list creators. Software tools for performing evaluations of word family lists such as AntWordProfiler (Anthony, 2008) are free and easily accessible, but the tools for evaluating a lemma list (with parts of speech identified) may need to be developed by the list maker in order assess a lemma-based list’s performance.

Nation (2016) states that the decision of unit of counting needs to be explicitly described and justified by a word list developer (p.39). If the level-6 word family (as shown in Section 2.3) is chosen as the unit of counting, the researcher should provide evidence that the target learners will be able to infer the meanings of derivational forms. Some word list developers such as Browne (2014) and Dang and Webb (2016) have chosen to base their lists on the lemma unit, but have not distinguished parts of speech (Browne described this as a "modified lemma”). In addition to providing a definition of a “word”, list makers should also clearly define their interpretation of a "lemma” when creating a lemma-based word list since this definition is an important factor to consider when evaluating and comparing word lists with different units of counting (further discussion of the necessary considerations when making list comparisons appear in Section 3.15). 
2. Word selection criteria

When establishing word selection criteria that will be used to extract the word list from the source corpus, word list developers may utilize quantitative measurements (such as frequency, range, and dispersion), more subjective criteria such as lexical sets (days of the week, family relationships, “survival vocabulary”), or a combination of both (Nation, 2016). Quantitative measurements have the advantage of making list development research replicable and allow for the sequencing of words in ranked orders of frequency, which should be of interest to list users seeking to maximize the efficiency of study time by prioritizing the most frequent items (Nation, 2001, 2016). More subjective criteria have the benefit of tailoring a word list toward the needs of the list users and including items relevant to the specific learning situation that might be otherwise excluded by solely quantitative measurements.

Of the quantitative measurements, word frequency has been the most widely used. Frequency refers to the number of times a word appears in the corpus, and is measured based on the chosen unit of counting (word family or lemma). A minimum frequency threshold is often set as a criterion for inclusion the word list. This minimum frequency threshold varies depending on the size of the corpus and the intended purpose of the word list. For the Academic Word List (Coxhead, 2000) this threshold was set to 100 counts of a word family in a corpus size of 3.5 million words based on a frequency analysis of the Brown Corpus (Francis \& Kučera, 1982). The threshold of 100 frequency counts has been also used by a number of academic and specialist vocabulary list studies with 
comparably sized corpora that have followed Coxhead's example (Minshall, 2013; Lei \& Liu, 2016; Wang et al, 2008).

The range measurement is used to count the number of texts or sub-corpora that a word appears in. In the case of an academic corpus divided into disciplinary sub-corpora such as “Science” or "Law”, items that have a high range value appeared in numerous disciplines and might be considered to representative of general academic vocabulary (not specific to a limited number of academic disciplines). In order to use the range criteria, Nation (2016) recommends that all sub-corpora are of similar size and are “internally coherent” (composed of similar types of texts).

Though items may have a low range value, they may still be highly frequent words in a corpus due to their appearances clustering within a limited number of sub-corpora. The measure of dispersion can be used to determine the distribution of a word throughout the corpus. In an academic corpus divided by disciplines, a low dispersion value could indicate that a word is a specialized vocabulary item that used more frequently within a certain discipline. While range and frequency are calculated by counting word occurrences, producing dispersion values involves a formula based on both range and frequency values. There are a number of possible methods for producing a dispersion value; studies of academic and specialist vocabulary such as Gardner and Davies (2014) and Lei and Liu (2016) have chosen Juilliand’s ‘d’, Browne (2014) selected Carroll’s 'D2', and Brezina and Gablasova (2015) chose to use Average Reduced Frequency (ARF) (Savický \& Hlavácová, 2002), which provides a frequency value that takes dispersion into account. Gries (2019) gives a comparison of the methods for producing dispersion 
values. Gries states that the various methods produce values that generally correlate with each other, but may the values may differ depending on the number of sub-corpora that a corpus is divided into. For the creation of the Computer Science Academic Vocabulary List (CSAVL), I selected Juilland's D as the measure of dispersion. I made this decision based on the example of and Lei and Liu (2016), who produced their lemma-based Medical Academic Vocabulary List from a medical corpus with a similar number of subcorpora to the computer science corpus that was used to create the CSAVL.

An additional approach to word selection has been the exclusion of content found in other word lists. For academic word lists that are designed to function as supplements to the General Service List (West, 1953), list makers have excluded GSL items from appearing in their own lists (Coxhead, 2000; Khani \& Tazik, 2013; Wang et al., 2008; Xue \& Nation, 1984). Minshall (2013) excluded both the contents of the GSL and AWL in the development of a specialist vocabulary list for the discipline of computer science. Exclusion of the contents of other word lists can provide a quick method for targeting a certain type of vocabulary, but also creates a relationship of dependency on the list which was used as the exclusion filter (Gardner \& Davies, 2014). The issues arising from dependency on lists such as the GSL are discussed further in Section 2.7.

\section{List evaluation}

Evaluations of a word list can be performed using quantitative measures such as a coverage test (where the percentage of the words in a corpus that match word list items is calculated), or using more qualitative measures such as the "Questions for critiquing a 
word list” provided in Nation (2016) (p. 131). When coverage tests are used to evaluate a word list, the results are compared with the coverage results of another list or lists. For the comparison to be a fair one, Nation (2016) recommends that the lists being compared have a similar purpose; have the same unit of counting, or that conversions of units are performed (e.g. word families are converted to lemmas); and that the difference in the number of words in each list is clearly described. Additionally, coverage tests should be performed using a corpus that was not the source corpus which was used to generate the word list (Miller \& Biber, 2015). When both the coverage percentage and the number of words is known, the efficiency of a list in terms of cost/benefit relationship may be determined. A word list that achieves the same amount of coverage as another list, but with fewer words, could be described as a more efficient list because it will likely result in a shorter time investment for learners. Because the lists that were compared in this study describe a similar range of vocabulary, but are of notably different sizes, normed coverage will be used a metric to determine each list's efficiency in covering a corpus of academic CS texts.

Though a word list may outperform another list in a coverage test, this does not necessarily indicate that it is able to meet the needs of the target users more effectively. To more thoroughly evaluate a list, evaluators can check word lists for the presence of desired sets of target vocabulary words (days of the week, numbers, colors), or use a qualitative approach such as the list of questions for critiquing a word list provided in Nation (2016). Nation's list of questions includes items such as "Was the purpose of the list clearly described?”, “Was the content of the corpus suited to the purpose of the list?”, 
and “Are the weaknesses of the list clearly acknowledged?”, which could provide the framework for a descriptive evaluation of a word list.

\subsection{Evaluations of GSL-based word lists}

In his 2001 book, Nation (who, along with Bauer, created the word family ranking system) cites West’s GSL as being a classic representation of the high-frequency word category. The GSL, one of the most widely-used word lists in English language education, is a list of 2000 headwords that were meant to represent the most frequently used words in the language. West did not clearly define a 'word' when creating the GSL and instead treated each word as "a separate problem” (West, 1953, p. viii), with the original GSL being a list of the forms of each headword that West considered to be the most essential. However, the list is now generally distributed as a list of word family headwords and has informed the creation of other word family-based lists. West's list, created in a pre-computer age and based on a corpus of texts from the early 1900's, has been criticized for its lack of representativeness of contemporary English due to its age and the relatively small corpus it was derived from (Browne, 2014; Gardner \& Davies, 2014; Lei \& Liu, 2016). As noted in Browne (2014), the aging GSL contains numerous items that are likely to be less relevant to $21^{\text {st }}$ century students of English, including nautical and agricultural terms (“vessel”, “mill”, “cultivator”), religious terms (“grace”), and terms affected by technological obsolescence (“telegraph”, “chimney”, “coal”). 
Additionally, the criteria for inclusion in the GSL was based on subjective criteria such as “ease of learning”, “necessity”, and “style”, which led to the inclusion of numerous lowfrequency items that might not be considered representative of a general high-frequency word list. The limitations of the GSL as a pedagogical tool for contemporary learners have led to the appearance of a number of “new” GSL lists in recent years (Brezina \& Gablasova, 2015; Browne, 2014).

Despite the issues with West’s GSL, a number of lists representing more specialized registers of English have used the GSL as a base to which their own word lists are added in order to achieve something closer to the 95\% lexical coverage (Hu \& Nation, 2000; Laufer, 1989; Laufer \& Ravenhorst-Kalovski, 2010) required for minimal comprehension of a text. Two of these GSL-supplementing lists representing academic words are Xue and Nation’s University Word List (UWL) (1984) and Coxhead’s AWL (2000). These researchers excluded the words on the GSL from their academic word lists in order to eliminate general high-frequency words from their lists. Nation and Xue created the UWL in 1984 by combining and editing four existing academic lists. 16 years later, Coxhead published the influential AWL. Coxhead found that in her corpus of academic texts, the GSL achieved 76\% coverage. When comparing the two GSL supplement lists (the UWL and AWL) using the same academic corpus, the 836-headword UWL achieved 9.8\% coverage, whereas her 570-headword AWL achieved 10\% coverage. Coxhead found her AWL to be a more efficient list than the UWL since it achieved similar coverage with far fewer headwords. 
Since the combination of the GSL and AWL achieved only $86 \%$ of the target $95 \%$ suggested for minimal comprehension, other researchers have attempted to bridge this comprehension gap by creating lists which represented discipline-specific technical word lists. These word family-based lists were designed as supplements to the AWL and excluded both the GSL and AWL words in their inclusion criteria. Most relevant to the present study is Minshall’s (2013) 433-headword Computer Science Word List (CSWL), which achieved $6 \%$ coverage in his own corpus of computer science texts. By combining the CSWL with the GSL and AWL, he was able to achieve Laufer's suggested target coverage of 95\% required for minimal comprehension. Although Minshall was able to achieve his target coverage, due to the CSWL's close relationship to the GSL and AWL, his list also inherited all of the problematic issues that came with each of these lists.

Issues regarding the AWL concept of a 'general' academic vocabulary were pointed out by researchers such as Hyland and Tse (2007), who argued instead for disciplinespecific sets of academic vocabulary. In tests of the AWL on their own academic corpus, they found that the coverage of the AWL was not evenly distributed across academic disciplines. In their study, the GSL+AWL provided 88\% coverage in the social sciences, while only providing $78 \%$ coverage in science texts. Of the 570 word families of the AWL, only 36 were relatively evenly distributed across their three categories of academic disciplines. In addition to coverage distribution issues in the AWL, when examining the AWL for the distribution of homographs (e.g. lead as a noun versus lead as a verb) across disciplines, they found that different disciplines "showed clear preferences for particular 
meanings and collocations” (p.244), which points to the need for academic lists to be discipline-specific in order to be as efficient as possible.

For the disciplinary focus of the present study, computer science, Hyland and Tse (2007) reported that the AWL provided excellent lexical coverage of academic CS texts (16\% coverage of CS texts in comparison with $6.2 \%$ of biology texts) (p.248), and similarly positive results were reported by Minshall (2013), who found the AWL to cover roughly $12 \%$ of his academic CS corpus. However, although lexical coverage may provide a convenient quantitative form of measurement, it might not be fully indicative of the relationship between a word list and the language usage in the register that it attempts to describe. Like Hyland and Tse (2007), Guo (2018), in an examination of ELLs studying computer programming, also found that many terms are used with different meanings in the context of CS, including AWL words such as instance as well as highfrequency GSL words such as for and while. These differences in meaning were discussed in an examination of novice programmer misconceptions related to natural language transfer performed by Bonar and Soloway (1985), who found that CS learners, based on natural language meanings, mistakenly assumed while to refer a continuously active test (a condition that is continuously evaluated). However, in typical programming, a while loop is only evaluated once per loop iteration, and this misconception resulted in logical errors in the code produced by these learners.

Lam (2001), in an examination of “sub-technical” vocabulary (words with both CSspecific meanings and general high-frequency meanings), also found that when reading CS texts, learners faced additional challenges which were linked to the transfer of 
general-usage meanings into a CS context, and when learners were provided with a technical dictionary of CS-specific meanings instead of a general-usage dictionary, achieved significantly higher scores in a series of CS-based reading comprehension tasks which specifically focused on these polysemous terms.

The overlap between words with CS-specific meanings and the contents of the GSL and AWL means that a CS vocabulary list which is built upon (and therefore excludes the contents of) the GSL and AWL word lists will be constrained by them and unable to indicate the special meanings found in the language of the academic computer science. As the studies described in this section suggest (Bonar \& Soloway, 1985; Guo, 2018; Lam, 2001), learners who have not examined the discipline-specific meanings of GSL and AWL items may be at a disadvantage and could face additional complications as a result of the transfer of general meanings to discipline-specific contexts. In order to include words with CS-specific meanings (such as “string”, “mouse”, and "volume”), the Computer Science Academic Vocabulary List (CSAVL) will not be built upon the GSL or AWL word lists, but will instead use other criteria to restrict the inclusion of general high-frequency words. Additionally, words with CS-specific meanings will be explicitly marked in the CSAVL for two reasons: 1) so that list users are made aware of differences between the use of these items in a CS context and a general-usage context; and 2) so that list users can easily find the appropriate CS-specific definition for these terms.

The GSL-based word lists that were described in this section are summarized for the reader's reference in Table 2.3. The next section describes lists which have addressed 
issues with the AWL and GSL in their design and which are more closely aligned with the methodology of the present study.

Table.2.3 The GSL and GSL-based word lists

\begin{tabular}{|c|c|c|c|c|}
\hline Word list & $\begin{array}{l}\text { Target form of } \\
\text { written language }\end{array}$ & Size & Supplement to & Coverage \\
\hline GSL (West, 1953) & $\begin{array}{l}\text { General high- } \\
\text { frequency English }\end{array}$ & $\begin{array}{l}\text { 2000 } \\
\text { headwords }\end{array}$ & - & $\begin{array}{l}76.1 \% \\
\text { (Coxhead's } \\
\text { corpus) }\end{array}$ \\
\hline $\begin{array}{l}\text { UWL (Xue \& } \\
\text { Nation, 1984) }\end{array}$ & Academic English & $\begin{array}{l}836 \\
\text { Word family } \\
\text { headwords }\end{array}$ & GSL & $\begin{array}{l}9.8 \% \\
\text { (Coxhead's } \\
\text { corpus) }\end{array}$ \\
\hline $\begin{array}{l}\text { AWL (Coxhead, } \\
\text { 2000) }\end{array}$ & Academic English & $\begin{array}{l}570 \\
\text { Word family } \\
\text { headwords }\end{array}$ & GSL & $\begin{array}{l}10 \% \\
\text { (own corpus) }\end{array}$ \\
\hline $\begin{array}{l}\text { MAWL (Wang et } \\
\text { al., 2008) }\end{array}$ & $\begin{array}{l}\text { Medical academic } \\
\text { English }\end{array}$ & $\begin{array}{l}623 \\
\text { Word family } \\
\text { headwords }\end{array}$ & GSL & $\begin{array}{l}12.24 \% \\
\text { (own corpus) }\end{array}$ \\
\hline $\begin{array}{l}\text { App. Ling. Acad. } \\
\text { Word List (Khani \& } \\
\text { Tazik, 2013) }\end{array}$ & $\begin{array}{l}\text { Applied Linguistics } \\
\text { Academic English }\end{array}$ & $\begin{array}{l}773 \\
\text { types }\end{array}$ & GSL & $\begin{array}{l}12.48 \% \\
\text { (own corpus) }\end{array}$ \\
\hline $\begin{array}{l}\text { CSWL (Minshall, } \\
\text { 2013) }\end{array}$ & $\begin{array}{l}\text { Computer science } \\
\text { academic English }\end{array}$ & $\begin{array}{l}433 \\
\text { Word family } \\
\text { headwords }\end{array}$ & GSL+AWL & $\begin{array}{l}6 \% \\
\text { (own corpus) }\end{array}$ \\
\hline
\end{tabular}

\subsection{Evaluations of lemma-based word lists}

The word list which has the strongest influence on the methodological design of the present study is Gardner and Davies’ (2014) Academic Vocabulary List (AVL). The 
design of the AVL differed from the lists described in the previous section in that 1) the AVL was not designed as a supplement to the GSL and 2) the AVL was based on the organizational unit of the lemma rather than the word family.

Regarding design decision number one, Gardner and Davies argued that the GSL (based on a corpus from the early 1900s) is no longer an accurate reflection of highfrequency English words. They also found that many high frequency words appeared more frequently in academic registers than general ones (words such as exchange), which led them to argue for the inclusion of these kinds of high-frequency words in their own academic word list. For the creation of the Computer Science Academic Vocabulary List, I also adopted this position in order to prevent the full variation of the register represented by the list from being restricted by the GSL. If high-frequency words such as memory take on unique technical meanings within a CS context, they should also be considered for inclusion on a CS academic vocabulary list.

In reference to the second design decision, the present study also adopts the use of the lemma rather than the word family due to a number of problematic aspects of the 6level word family that have been described by numerous researchers (Gardner, 2007; Gardner \& Davies, 2014; Hyland \& Tse, 2007; Wang \& Nation, 2004). A word family can include variations of a headword across parts of speech, so the family headed by proceed will also contain both the verb proceed and the noun proceeds, despite the distinctly different meanings of the two forms. Word family headwords can lead to misrepresentative frequency counts when searching a corpus (proceed as a verb may be a high frequency word, while proceeds as a noun may not, yet the occurrences of proceeds 
as a noun will still be included in the total count). Wang and Nation, in their analysis of Coxhead's word-family based AWL, found that one in ten of the word families in the AWL contained homographs such as proceed. Their findings, along with those of Hyland and Tse, regarding the uneven distribution of homographs across disciplines also suggest that word family-based lists are less efficient for learners since they can prompt them to spend time studying meanings of a headword that may be infrequently used in their intended academic discipline. The move to a lemma-based list mitigates these issues since different parts of speech appear as different list entries (proceed as a verb will appear separately from proceed as a noun).

Gardner (2007) also pointed out an additional problem with 6-level word families: inflectional knowledge comes sooner than derivational knowledge for ELLs. It may be problematic to assume that learners entering EAP programs will have enough derivational knowledge to recognize the relationship of a headword to derivational forms up to level 6 (e.g. the relationship between react and reactor). Furthermore, researchers have pointed out how learners from L1 backgrounds with different morphological systems may face additional challenges when analyzing these derivational forms (Corson, 1997; Ward, 2009). These derivational transparency issues for ELLs can be addressed by lemma-based lists, which group headwords only with inflected forms and items such as react and reactor have separate entries.

Additional support for the development of lemma-based word lists has been provided by researchers who have created lemma-based replacements for the GSL. Browne’s (2014) New General Service List (NGSL) and Brezina and Gablasova’s New- 
GSL (2015) are both lemma-based general word lists that have demonstrated higher coverage with fewer words in comparisons with West's GSL. A lemma-based approach was also taken by Lei and Liu (2016) in the development of their discipline-specific Medical Academic Vocabulary List (MAVL), which provided more coverage than the word family-based MAWL (Wang et al., 2008). When both lists were compared in lemma form, the MAVL not only achieved superior coverage, but did so with far fewer lemmas (the MAWL contained 1751 lemmas compared to the MAVL's 819). When list size is compared along with coverage, it can give an indication of the degree of efficiency for a given list. The findings of the researchers who created the NGSL, New GSL, and MAVL all suggest that, in addition to being more informative and user-friendly than word-family lists, lemma-based lists are also more efficient. The lemma-based word lists described in this section are summarized here for the reader's reference in Table 2.4. 
Table 2.4 Lemma-based word lists

\begin{tabular}{lllll}
\hline Word list & $\begin{array}{l}\text { Target form of written } \\
\text { language }\end{array}$ & Size & Supplement to & Coverage \\
\hline $\begin{array}{l}\text { AVL } \\
\text { (Gardner \& }\end{array}$ & Academic English & 3,000 & - & $13.8 \%$ \\
Davies, 2014) & & lemmas & $\begin{array}{l}\text { (top 570 headwords of } \\
\text { AVL in COCA } \\
\text { academic) }\end{array}$ \\
NGSL & General high-frequency & 2,818 & - & $90 \%$ \\
(Browne, & English & modified & (CEC corpus) \\
2014) & & lemmas* & \\
New GSL & General high-frequency & 2,494 & - & $80.3 \%$ \\
(Brezina and & English & lemmas & (BNC corpus) \\
Gablasova, & & & \\
2015) & & & $19.44 \%$ \\
MAVL (Lei & Medical academic & 819 & (own corpus) \\
\& Liu, 2016) & English & lemmas & \\
\hline
\end{tabular}

* The modified lemma is described in Section 3.15.

COCA: The Corpus of Contemporary American English (Davies, 2008)

CEC: The Cambridge English Corpus

BNC: The British National Corpus

\subsection{The Computer Science Academic Word List (Minshall, 2013)}

Minshall’s (2013) Computer Science Academic Word List (CSWL) was created with a purpose similar to the subject of this thesis (the Computer Science Academic Vocabulary list) in that it attempted to create a pedagogical tool for computer science students enrolled in English for Specific Purposes (ESP) instruction. However, the CSWL was based upon the word family unit and created as a technical supplement to both the General Service List (West, 1993) and the Academic Word List (Coxhead, 
2000). This choice resulted in the CSWL having a number of characteristics that make it substantially different from the project of this thesis (the CSAVL).

The CSWL was derived from a 3.6-million-word corpus designed by Minshall which contained 408 texts and was split into two sub-corpora based on text types: academic journal articles and academic conference proceedings. The two main subcorpora were each split into 10 CS sub-disciplinary categories that were based on the publication classification system of the Association of Computing Machinery (the source of all texts that were included in the corpus).

Minshall set three criteria for inclusion the CSWL: 1) specialized occurrence, 2) range, and 3) frequency. The specialized occurrence criterion was designed to filter general high-frequency and general academic words from the list. To do this, Minshall excluded all word families appearing in the both the GSL and AWL from his list. The second criterion, range, specified that word families had to appear in at least five out of the ten sub-disciplinary categories in the corpus. The third criterion, frequency, specified that word families had to appear at least 80 times to be included in the word list.

When evaluating the CSWL, Minshall found that it achieved 6\% coverage in the source corpus that was used to generate the list, and $4.8 \%$ coverage in a secondary test corpus that included both CS journal articles and textbooks. When combining the CSWL with the GSL and AWL, he was able to achieve his target of 95.11\% coverage of the source corpus, and achieved $94.41 \%$ coverage of the secondary test corpus.

As a result of the exclusion of GSL word families, Minshall prevented highfrequency polysemous GSL words with CS-specific meanings (such as “memory” and 
“class”) from being included in the CSWL. By excluding academic AWL words from the technical CSWL list, Minshall may have disregarded the overlap between the categories of academic vocabulary and technical vocabulary. AWL academic vocabulary items such as "core” and "function” also take on discipline-specific meanings within a CS context and restricting items like these from the CSWL limits it from representing the full range of the register. Additionally, since Minshall based the CSWL on the word family unit, learners are assumed to be able to recognize the relationship of each headword to its derivational forms, which is an assumption that has been challenged by numerous researchers (Brezina \& Gablasova, 2015; Corson, 1997; Dang \& Webb, 2016; Gardner, 2007; Gardner \& Davies, 2014; Ward, 2009).

The CSWL (see Figure 2.2 for a sample) does not specify part of speech for list items and gives no indication of whether or not an item has a discipline-specific meaning. This lack of discipline-specific information reduces list efficiency and may create additional complications (as was observed in Lam, 2001) for learners who may spend time studying parts of speech or meanings of a headword that are infrequently used in academic computer science (“bug” as an insect, "port” as a city with a harbor) and mistakenly assume that those meanings can be applied to a CS context. Additionally, the CSWL provides no frequency rankings for list items. By not including frequency rank information, the CSWL does not fully allow list users to prioritize the study of vocabulary based on frequency of use, which is one of the primary purposes of a word list. 


\begin{tabular}{|llll|}
\hline \multicolumn{4}{|l|}{ Headwords of the CSWL in alphabetical order } \\
\hline accelerate & activate & acyclic & adversary \\
affine & afore & algebra & algorithm \\
align & alphabet & amortise & annotate \\
anomaly & anonymous & arc & architecture \\
arithmetic & array & artifact & asynchronous \\
atom & audio & augment & authentic \\
authorise & automaton & autonomic & auxiliary \\
avail & axis & backup & bandwidth \\
barrier & baseline & batch & battery \\
bayesian & benchmark & binary & binomial \\
bitmap & boolean & bottleneck & breakdown \\
browse & budget & buffer & bug \\
\hline
\end{tabular}

Figure 2.2 A sampling of the Computer Science Word List (CSWL) (Minshall, 2013)

\subsection{Research Purposes of the CSAVL project}

Although there is an existing word family-based computer science word list (Minshall’s 2013 CSWL), there has not been a lemma-based CS word list which attempts to represent the broader academic vocabulary of the discipline, identifies items with CSspecific meanings, identifies parts of speech for list items, or incorporates the technologies of corpus part-of-speech tagging and statistical analysis. In order to develop a word list that can better meet the needs of ESL learners bound for study in the context of academic computer science, I would like to propose the following list of ideal characteristics for the Computer Science Academic Corpus (CSAC): 
(1) The corpus should contain at least 3.5 million tokens and be balanced in size across each sub-corpus. Each sub-corpus should only contain a type of text (e.g. journal articles).

(2) In order to be representative of the discipline, the corpus should include texts which are clear examples of academic computer science writing and includes as many sub-disciplinary topics and authors as possible.

(3) Texts within the corpus should also be as recent in order to be representative of the population of texts that are currently in actively in use within the discipline.

In addition to these corpus characteristics, I propose the following list of ideal characteristics for a discipline-specific computer science academic vocabulary list (CSAVL):

(1) The list should be lemma-based in order to be as representative as possible of the register and maximize efficiency for its users by guiding them toward the specific parts of speech for each item that are most frequently found in academic CS texts.

(2) In order to more fully describe the variation of this register, the list should not exclude items appearing on a general service list, but instead use other criteria to exclude general high-frequency words in order to acknowledge that specialist vocabulary can be found within all frequency bands of vocabulary. 
(3) The list should explicitly identify items that have CS-specific meanings so that learners can focus on the meanings that are most relevant to their chosen discipline.

After creating the Computer Science Academic Corpus and the Computer Science Academic Vocabulary List, the primary purposes of this research project will be as follows:

1. To compare the coverage and content of the CSAVL with the Academic Word List (Coxhead, 2000) and the Minshall (2013) Computer Science Word List (CSWL).

2. To determine the amount of coverage of an academic CS corpus that can be achieved by combining the CSAVL with a lemma-based general high-frequency word list such as the new-GSL (Brezina \& Gablasova, 2015).

3. If the combination of the CSAVL and new-GSL do not reach the 95\% lexical coverage threshold suggested by Laufer $(1989,2010)$ for a minimal level of reading comprehension, to create a supplement to the CSAVL in order to reach this target. 


\section{Chapter 3: Methodology}

The first half of the methodology chapter (Sections 3.1 to 3.7) describes the methods employed during the creation of the Computer Science Academic Corpus 1 (CSAC1) and the test corpus, the Computer Science Academic Corpus 2 (CSAC2). This includes a corpus design overview (Section 3.1), an explanation of the type of texts that were selected (Section 3.2), the criteria that were established to select specific articles and textbooks (Section 3.3 and 3.4), a description of corpus size and balance (Section 3.5 and 3.6), and explanations of the text processing procedures (Sections 3.7 and 3.8).

The second portion of the methodology chapter (from Sections 3.9 to 3.13) focuses on the methods utilized in creating the Computer Science Academic Vocabulary List (CSAVL) and its supplemental list (the CSAVL-S). This second half of the chapter contains the criteria for word list inclusion (Section 3.9), descriptions of the tokenization and tagging process (Section 3.10), the technique used to extract the CSAVL and CSAVL-S lemmas (Sections 3.11 and 3.12), and the method utilized when making additions to the initial lists (Section 3.13). Section 3.14 details the content which was removed from the CSAC2 test corpus before word list coverage tests were performed, and Section 3.15 describes how comparisons between word lists of differing structural organizations were performed. The methodology chapter concludes with a summary in Section 3.16. 


\subsection{Corpus design overview}

In order to develop the Computer Science Academic Vocabulary List (CSAVL), two corpora of academic CS texts were developed: The Computer Science Academic Corpus 1 and 2 (CSAC1 \& CSAC2). Since a word list derived from a given corpus is to some degree a frequency measure of the items in that corpus, it will likely have an unfairly high lexical coverage percentage of that source corpus (Coxhead, 2000; Nation, 2016). Therefore, it is also important to develop a second corpus to make a more valid coverage test that might demonstrate its representativeness of texts across the discipline. This practice was employed by both Coxhead and Minshall, and a similar strategy was developed by Gardner and Davies (2014), who used the British National Corpus (BNC) to make coverage evaluations of their Corpus of Contemporary American English (COCA) -derived AVL. In this study, the larger CSAC1 corpus was used to generate the CSAVL word list and the smaller CSAC2 corpus was used to evaluate the word list (this two-stage process is depicted here in Figure 3.1). 


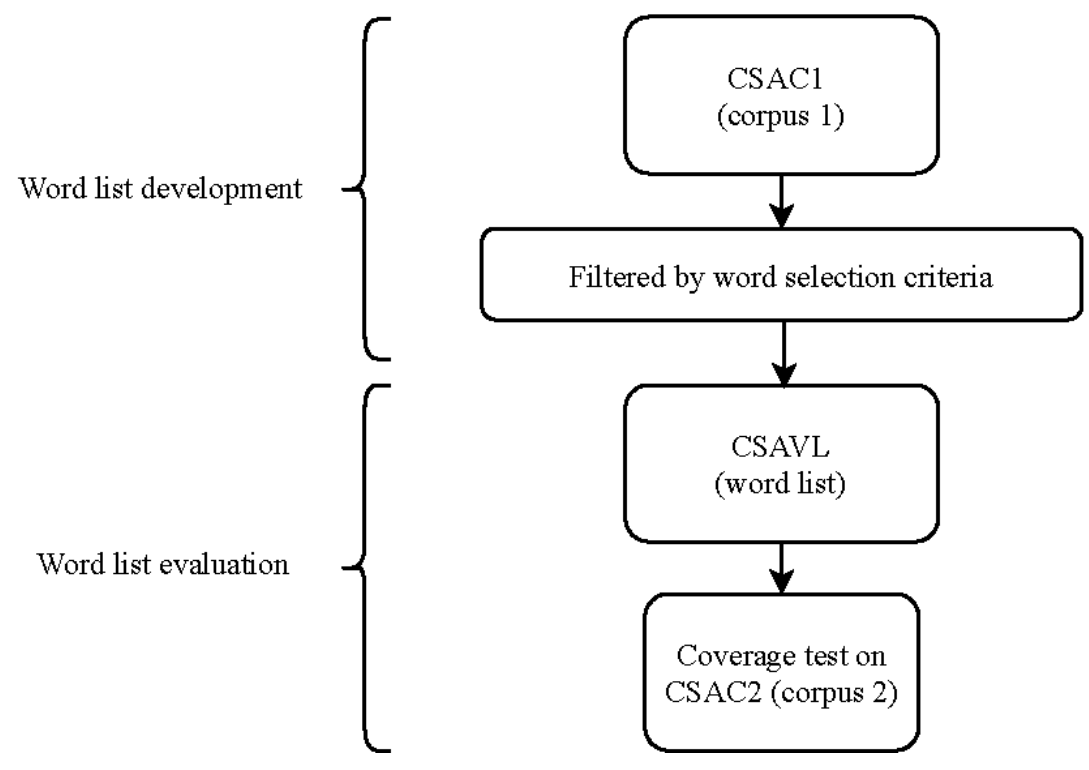

Figure 3.1 Word list development flowchart

The CSAC1 and CSAC2 corpora were developed in the following sequence:

\section{Establishment of corpora sizes}

The CSAC1 and CSAC2 corpora were designed to be as parallel in organization as possible, with the primary difference being the number of tokens that were included in each sub-corpus. For the larger CSAC1, which would be used to derive the CSAVL, a corpus size of 3.5 million tokens was chosen. This size choice matches that of the source corpora used to derive both the Academic Word List (AWL) (Coxhead, 2000) and the Computer Science Word List (CSWL) (Minshall, 2013). The CSAC1 corpus was designed to be comprised of two equal-sized parts, each with a target number of 1.75 million tokens of text. The first 1.75-million-token half was allocated for ACM journal articles, and the second 1.75 million token half for CS textbooks. For the smaller CSAC2 
test corpus, a target size of 700,000 tokens was set. This is similar to the size of the test corpora used by both Coxhead (2000) and Minshall (2013) in their evaluations of their respective word lists. Table 3.1 outlines the planned structure for the CSAC1 source corpus and the CSAC2 test corpus.

Table 3.1 Planned structure of CSAC1 and CSAC2

\begin{tabular}{ll|ll}
\hline & \multicolumn{2}{c}{ CSAC2 } \\
\hline Text type & Target token & Text type & Target token \\
& count & & count \\
\hline Textbooks & $\sim 1,700,000$ & Textbooks & $\sim 350,000$ \\
Journal Articles & $\sim 1,700,000$ & Journal Articles & $\sim 350,000$ \\
\hline CSAC1 Total: & $\sim 3,500,000$ & CSAC2 Total: & $\sim 700,000$ \\
\hline
\end{tabular}

2. Determining the target size and number of sub-corpora

To establish the number of sub-corpora in both CSAC1 and CSAC2, the two text types (journal articles and textbooks) were divided into 10 categories based on computer science sub-disciplines. The method that was used to determine these 10 sub-disciplinary categories was based on Minshall's (2013) method for setting sub-corpora categories for the corpus used to derive the CSWL, and is described in detail in Section 3.2. After the two text types were organized into 10 topical categories, a total of 20 sub-corpora was the result. For the CSAC1, each of the 20 sub-corpora included a target number of 175,000 tokens $\left(1 / 20^{\text {th }}\right.$ of the total corpus size), and for the smaller CSAC2, each of the 20 sub- 
corpora included a target number of 35,000 tokens $\left(1 / 20^{\text {th }}\right.$ of the CSAC2). These planned values are shown below in Table 3.2.

Table 3.2 Planned sub-corpus structure of CSAC1 and CSAC2

\begin{tabular}{ll|ll}
\hline CSAC1 & Tokens & CSAC2 & Tokens \\
\hline $\begin{array}{l}\text { 10 Sub-disciplinary } \\
\text { journal article categories }\end{array}$ & $\sim 175,000$ each & $\begin{array}{l}\text { 10 Sub-disciplinary } \\
\text { journal article categories }\end{array}$ & $\sim 35,000$ each \\
\hline $\begin{array}{llll}\text { Articles Total: } \\
\text { 10 Sub-disciplinary }\end{array}$ & $\sim 17, \mathbf{5 0 , 0 0 0}$ & Articles Total: & $\sim \mathbf{3 5 0 , 0 0 0}$ \\
textbook categories & $\sim \mathbf{1 , 7 5 0 , 0 0 0}$ & Textbooks Total: & $\sim 35,000$ each \\
Textbooks Total: & $\begin{array}{l}\text { 10 Sub-disciplinary } \\
\text { textbook categories }\end{array}$ & $\sim \mathbf{3 5 0 0 0}$ \\
\hline CSAC1 Total: & $\sim \mathbf{3 , 5 0 0 , 0 0 0}$ & CSAC2 Total: & \\
\hline
\end{tabular}

\section{Establishing text selection criteria}

After determining a source for CS journal articles and performing a survey of CS textbooks currently in use in American universities (both of these processes are fully described in Section 3.2 below), three selection criteria were set for choosing the texts that would be included in the CSAC1 and CSAC2 corpora. These criteria were established for the purpose of selecting texts which best represented the population of texts that might be encountered by undergraduate CS students studying in American universities in 2019. For journal articles, the three criteria for selection were topical relevance, recency, and impact and usage. For textbooks the selection criteria were 
topical relevance, institutional influence, and current usage. The definitions and operationalization of these criteria are described in Sections 3.3 and 3.4.

4. Balancing token counts within the sub-corpora

Once the criteria for selection had been applied, an amount of data exceeding the target word counts were collected in order to allow for the removal of texts that were found to be what Sinclair (2005) described as "rogue” texts; texts which stand out as radically different (in size or content) from other texts in the category. To reach the target token counts for each sub-corpus, a mean token count for all of the collected texts in each category of articles/book chapters was determined. Texts which greatly deviated from that mean count were removed when selecting the final texts that would result in the target token count. In the sub-corpora of CSAC2, which had a target token count of 35,000 tokens each, a hypothetical book chapter made up of 60,000 tokens would be excluded for both exceeding the target token count and preventing the inclusion of other texts in that sub-corpus. Of the 24 journal articles that were collected to represent each category, from one to five journal articles were removed due to their size difference from the mean token count for that category (more than 15,000 tokens). Of the 22 textbooks that were collected, I removed chapters from four textbooks due to irregular size (deviation from the mean size of more than 35,000 tokens). I also examined both journal articles and textbook chapters for the presence of "radically different" content (content that was only found a single text and based on my own knowledge of CS, was not 
representative of the discipline) during the processing of the texts, but no noticeably irregular content was found or removed.

\section{Data processing}

After identifying the specific texts that would make up each sub-corpus of the CSAC1 and CSAC2, the final data were processed. This began with a phase of manual processing where images and other non-essential elements of the digital texts were removed (described in Section 3.7) before the automated processing phase, where regular expressions were used and programs were written in order to prepare the text for analysis (described in Section 3.8). Once the data had been fully prepared, tokenization, lemmatization, and part-of-speech tagging could be performed on the corpora (described in Section 3.10).

\subsection{Text types}

Both corpora are collections of academic computer science texts that were selected to represent the kinds of written language an undergraduate CS student, studying at an American university in 2019, might encounter. The vocabulary of the "core” CS classes, required courses that are taken in the initial years of study in a CS program, is represented by the CS textbook portion of the corpora. The core CS classes often utilize a 
textbook and it was found that certain texts were utilized by multiple universities (as described later in this section). The vocabulary of the upper-division CS coursework, which is far more varied in the topics represented and can include independent research projects, is represented by the content of the academic journal article portion of the corpora. These articles were selected based upon recency, impact, and usage in order to represent the vocabulary that students may encounter when engaging in guided or independent research projects.

The determination of the text types and text categories for the Computer Science Academic Corpus (CSAC1 and CSAC2) began by examining the methodology that was used by Minshall (2013) in order to create the corpus which was used to derive the Computer Science Word List (CSWL). When exploring possible text types for inclusion in his corpus, Minshall conducted interviews with university lecturers, who recommended textbooks, journal articles, special interest group newsletters and conference proceedings. Minshall chose not to incorporate textbooks in his corpus since he was concerned that large texts produced by a single author would introduce idiosyncratic author bias into the data. For this project, I have chosen to include textbooks for two reasons. First, textbooks are likely to be frequently encountered by undergraduate CS students, especially in their initial years at universities. Second, the multiple rangebased criteria established for inclusion in the CSAVL should account for author bias, since only words appearing across a range of texts will be included. Newsletters, conference proceedings, course-specific handouts, and other materials were not included 
in order to limit the scope of this project, though they might be valid candidates for inclusion in a larger-scale academic CS corpus.

In order to categorize the texts in the corpus, I followed Minshall's example of organizing journal texts using the Association of Computing Machinery’s (ACM) Computing Classification System (CCS). The ACM and the IEEE Computer Society (Institute of Electrical and Electronics Engineers) maintain two of the largest collections of CS journals in electronic form. The ACM uses their classification system to flag journal articles as being relevant to 11 sub-disciplinary categories. Minshall chose to omit one of these (Applied Computing) since it related to computing in other domains and only made use of the remaining 10 categories (listed here in Table 3.3). Since this system provides a convenient categorization system for classifying texts into sub-disciplines, I also chose to adopt this system and source my journal articles from the ACM's online library.

Table 3.3 . Journal Article Categories

\begin{tabular}{ll}
\hline \multicolumn{1}{c}{ Journal article categories } \\
(based on the ACM Computing classification system) \\
\hline 1. Computer systems organization & 6. Mathematics of computing \\
\begin{tabular}{ll} 
2. Computing methodologies & 7. Networks \\
3. Hardware & 8. Security and privacy \\
4. Human-centered computing & 9. Software and its engineering \\
\hline 5. Information systems & 10. Theory of computation \\
\hline
\end{tabular}
\end{tabular}


Before textbooks categories were determined, I performed a systematic survey of CS textbooks currently used in core CS courses at 21 American universities: the first 20 being the universities that were determined to have the most influential CS departments in the US (as described in the next paragraph); and the $21^{\text {st }}$ being the university where this thesis research was conducted, Portland State University (PSU). A “core” CS course is defined here as a course that is required by the department and is not an elective (program requirements where there were two or more possible course options were considered to be “electives”). Textbooks for electives courses were not chosen for inclusion for two reasons: First, the large number of CS electives found at the universities under examination was beyond the practical scope and resources of this project. Second, the elective courses were found to more closely align with the topics and content of the ACM research articles (hardware, operating systems, security, networks, and information systems) than those of the core course textbooks. This topical alignment of topics between the ACM articles and the elective courses informed the decision to allow the lexicon of the upper-level CS coursework to be exemplified by the content of the journal articles.

The initial survey of textbook usage began with identifying 20 universities to examine. Since influential and esteemed CS departments may be looked to as models when selecting English-language textbooks at universities throughout the U.S., it was decided that this would be the starting point for the survey. Google searches for "best computer science universities” featured the top result of the U.S. News \& World Report rankings (U.S. News \& World Report, 2018). Since this list was generated from surveys 
of department heads and directors of graduate studies in CS, it was decided to also examine a second ranking system, csrankings.org (Berger, 2016), which generated their list based on the number of Google Scholar citations received by faculty at each university. The rankings on the two sites were similar in their top 10 selections and began to diverge beyond that rank. The final 20 universities chosen for the survey were the top 20 scores based on the mean of their usnews.com rank and csrankings.org rank. A listing of these mean ranks can be found here in Appendix C. In addition to this ranked list of 20, the CS textbooks used at Portland State University (the institution at which this research was conducted), were also examined. This addition was made due to the likelihood that the resulting CSAVL list would be used in order to develop CS-specific EAP materials for use in the Intensive English Language program at PSU.

After generating the final list of 21 universities, each CS department website was examined and a listing of the required "core" courses (as earlier defined) at each school was made. The syllabus or material requirements for each of these courses was found on course-related websites and the textbook and other materials were recorded in spreadsheet entries. Appendix C also includes this listing and the corresponding list of approximately 200 textbooks that were found. Based on these data, 10 categories of core CS textbooks were created that were intended to mirror the categories used for the ACM journal articles as closely as possible. Table 3.4 lists the categories used to organized the textbook portions of the CSAC1 and CSAC2 corpora. The categories which I devised specifically for the textbook sub-corpora have been flagged with an asterisk. 
Table 3.4 Textbook categories

\begin{tabular}{ll}
\hline \multicolumn{2}{c}{ Core CS Textbook categories } \\
\hline 1. Computer systems organization & 6. Mathematics of computing \\
\begin{tabular}{ll} 
2. Data structures* & 7. Programming* \\
3. Algorithms* & 8. Operating systems* \\
4. Human-centered computing & 9. Software and its engineering \\
\hline 5. Probability and statistics* & 10. Theory of computation \\
\hline
\end{tabular}
\end{tabular}

The ACM categories that were replaced (computing methodologies, networks, hardware, security \& privacy, and information systems) better corresponded with elective courses and did not reflect the common core courses in most of the CS departments examined. In this list, "Human-centered computing” includes topics such as computing and society and the ethics of computing in order to better fit the types of core CS courses found. Although social or ethical computing courses did not appear in the majority of core curricula, it was still considered an important topic for inclusion because of its appearance in the Portland State University curriculum, where the needs of the English for Academic Purposes (EAP) program helped to motivate the creation of the CSAVL.

After establishing these 10 textbook categories, counts were made of the number of times each textbook was used in the list of examined courses. The usage frequency counts ranged from 13 (out of 20 universities) to single usages for two of the categories (these counts appear here in Appendix D). The two most frequently used (in 2019) textbooks which represented each of the 10 categories were selected for possible 
inclusion in the CSAC corpora. In most cases, a single textbook was enough to meet the target word count for a CSAC1 sub-corpora, and the second textbook was instead used in the CSAC2 test corpus.

\subsection{Journal article selection criteria}

Specific ACM journal articles were selected for inclusion in the CSAC1 and CSAC2 corpora based on three criteria:

\section{Topical relevance}

The first criterion was chosen to ensure that the contents of the articles were representative of their sub-corpora category. In order to meet the first criterion (topical relevance) a journal article’s primary sub-disciplinary ACM CCS tag had to match the sub-corpus it would be included in (i.e. articles included in the 'Hardware' sub-corpus must be tagged with 'Hardware' as the primary ACM CCS tag).

\section{Recency}

The second criterion (recency), was set in order to for the list to represent a quickly evolving and linguistically productive discipline where neologisms were not uncommon. In order to meet this criterion, articles had to be recently published, with a maximum age of 10 years since date of publication. This allowed for the possible inclusion of more newly established lexical items in the corpus such as “crowdsource”. 


\section{Impact and usage}

The third criterion (impact and usage) was established so that articles which were likely to be the most widely read and might the generate the greatest academic interest would be included. To operationalize this criterion, ACM journal articles which had the received the most unique downloads (downloads from unique IP addresses) from the ACM digital library received preference for inclusion.

The choice to use download counts as a criterion for inclusion was informed by research which has examined the relationship between download and citation counts (Schloegl \& Gorraiz, 2011; Watson, 2009), and research which has pointed out the utility of download counts as a metric of impact for recently published work (Wan et al., 2010). Watson, finding a strong positive correlation between total download counts and total number of citations, also found that citation rates lagged behind download counts by several years. This led the researcher to suggest that download statistics could be used as an indicator of eventual citations. Similar findings were produced by Schloegl and Gorraiz (2011), who reported a strong correlation between citations and downloads when absolute values were used. Additionally, Wan et al. (2010) have proposed a download immediacy index (DII) in order to estimate the impact of an article when its citation history is short. This is the case for many of the articles that were included in CSAC1 and CSAC2, which had been recently published and represented topics currently in demand, but whose history had been too short to accrue citation counts. The advanced search 
features of the ACM digital library allowed the three article criteria (primary CCS tag, publication within the last 10 years, and number of unique downloads) to be selected and articles were drawn from the top search results.

\subsection{Textbook selection criteria}

CS textbooks were chosen for inclusion in the CSAC1 and CSAC2 corpora based on the following three criteria:

\section{Topical relevance}

The relevance criterion was chosen to ensure that the contents of the textbooks were representative of their sub-corpora category, which was that of a core CS course that could be found at many American universities. To be included as a representative of a “programming” course, a textbook had to contain contents primarily related to the topic of computer programming and be in use in at least one core CS course that fit the programming sub-corpora category (a core CS course focused on basic programming syntax and concepts).

2. Institutional influence

The institutional influence criterion was set in order to included textbooks which were used in universities that could be influential within the discipline and whose 
textbook selections may be used to inform the textbook selections of other universities across the US. All texts that were considered for inclusion had to be used by one of the “top 20” US computer science programs (the method of identifying these schools was described in Section 3.2).

\section{Current usage}

The third criterion was designed to include texts which were most frequently used by the universities that were examined. This was done with the assumption that the sample of 21 universities may be somewhat representative of the population of all US universities with a CS program. Texts with the highest usage counts were included in the CSAC1 source corpus, and texts with the second or third highest counts were usually included in the CSAC2 test corpus (depending on the target token count requirements for each corpus).

\subsection{CSAC1 size and balance}

Once a collection of texts that met all criteria had been collected, the task of balancing token counts within each corpus and sub-corpus was performed. As noted by Nation (2016), when using the range criterion to extract words from a corpus, each subcorpus should be of the same size in order to prevent distorted results. Since several of the CSAVL word list inclusion criteria relied upon range measures, it was important to 
maintain this token count balance across all sub-corpora. For the CSAC1 corpus that would be used to derive the CSAVL word list, a total of 12 textbooks and 142 journal articles were included, and the resulting token counts after the data collection and processing stages are shown here in Table 3.5. Efforts were made during these stages to keep the final token counts as close as possible to the original token targets of 1.75 million tokens for each text type and 3.5 million tokens for the combined corpus.

Table 3.5 Overall makeup of CSAC1

\begin{tabular}{lll}
\hline Text type & No. of texts & Tokens \\
\hline Textbooks & 12 & $1,773,522$ \\
ACM Journal Articles & 142 & $1,758,934$ \\
CSAC1 Total: & $\mathbf{1 5 4}$ & $\mathbf{3 , 5 3 2 , 4 8 6}$ \\
\hline
\end{tabular}

As was described in Section 3.1, each 1.75-million-word half of CSAC1 was composed of 10 categories. The ACM articles were grouped into 10 categories based on the ACM sub-disciplines, while the textbooks were grouped into the 10 similar categories described in Section 3.2. This resulted in a total of 20 sub-corpora (10 categories of textbooks and 10 categories of articles) with a target figure of 175,000 tokens for each of the 20 sub-corpora.

Minshall (2013), when developing his own academic CS corpus, found that the ACM journal articles that he had collected averaged to approximately 11,000 tokens in length. Using this figure as a guideline, an amount of data that exceeded the target word counts were collected in order to allow for the later removal of any problematic texts. 
This excess quantity of ACM articles (which had been selected using the criteria described in Section 3.3) was then examined for individual token counts. The initial sampling of articles ranged in size from 4,000 to 33,000 tokens. In order to include a more even sampling of authors across each category, the largest and smallest of these articles were removed. In order to accomplish this, the mean token count for all the articles that had been collected for each category was calculated. Only the articles which were closest to the category mean count were selected for inclusion in the corpus. Table 3.6 outlines the final word counts of the journal article portion of CSAC1.

Table 3.6 Journal article makeup of CSAC1

\begin{tabular}{llll}
\hline Text type & No. of texts & Tokens & $\begin{array}{l}\text { Mean token } \\
\text { count per } \\
\text { article }\end{array}$ \\
\hline ACM Journal Articles & & & \\
\hline 1. Computer systems organization & 13 & 176,171 & 13,552 \\
\hline 2. Computing methodologies & 15 & 177,991 & 11,866 \\
\hline 3. Hardware & 15 & 176,957 & 11,797 \\
\hline 4. Human-centered computing & 16 & 176,177 & 11,011 \\
\hline 5. Information systems & 11 & 175,230 & 15,930 \\
\hline 6. Mathematics of computing & 17 & 175,202 & 10,306 \\
\hline 7. Networks & 13 & 175,071 & 13,467 \\
\hline 8. Security and privacy & 12 & 175,253 & 14,604 \\
\hline 9. Software and its engineering & 12 & 175,723 & 14,644 \\
\hline 10. Theory of computation & 18 & 175,159 & 9,731 \\
\hline Articles Total: & $\mathbf{1 4 2}$ & $\mathbf{1 , 7 5 8 , 9 3 4}$ & $\mathbf{1 2 , 3 8 7}$ \\
\hline
\end{tabular}


For the textbook portion of CSAC1, I divided textbooks into chapters in order to reach a final token count as close as possible to the target number of 175,000 tokens for each sub-corpus. The decision to divide textbooks into chapters rather than sample every nth page was made in order to reduce the number of partial sentence fragments into the corpus. This was a concern since the corpus would be part-of-speech tagged and fragments would be likely to reduce the accuracy of the part of speech tagger. Additionally, nth page sampling may have resulted in repeatedly sampling from homework exercises due to the repeated organizational structure of each chapter. This could have distorted the sampling and would have likely been a less optimal method of capturing the full variety of language used throughout the text. When selecting chapters, preference was given to chapters which occurred earlier in each book with the reasoning that earlier chapters of textbooks can represent fundamental concepts that learners may be more likely to encounter than those presented in the latter chapters that build upon those fundamentals. The final token counts for the textbook portion of CSAC1 is listed in Table 3.7. Overall, 12 textbooks were sampled from and 142 full ACM articles were included in the CSAC1. Appendix E contains a more detailed listing of each article and textbook chapter included in the corpus along with token counts for individual texts. 
Table 3.7 Textbook makeup of CSAC1

\begin{tabular}{|c|c|c|c|}
\hline Text type & No. of texts & Tokens & $\begin{array}{l}\text { Mean token } \\
\text { count per } \\
\text { chapter }\end{array}$ \\
\hline \multicolumn{4}{|l|}{ Textbooks } \\
\hline $\begin{array}{l}\text { 1. Computer systems } \\
\text { organization }\end{array}$ & $\begin{array}{l}1 \\
\text { ( } 5 \text { of } 7 \text { chapters) }\end{array}$ & 175,601 & 35,120 \\
\hline 2. Data Structures & $\begin{array}{l}1 \\
\text { (12 of } 12 \text { chapters) }\end{array}$ & 179,794 & 14,983 \\
\hline 3. Algorithms & $\begin{array}{l}1 \\
\text { (19 of } 20 \text { chapters) }\end{array}$ & 174,809 & 9,200 \\
\hline 4. Human-centered computing & $\begin{array}{l}1 \\
\text { (9 of } 10 \text { chapters) }\end{array}$ & 177,316 & 19,702 \\
\hline 5. Probability \& Statistics & $\begin{array}{l}2 \\
\text { ( } 4 \text { of } 4 \text { ) and (3 of } 7 \text { chapters) }\end{array}$ & 178,290 & 25,470 \\
\hline 6. Mathematics of computing & $\begin{array}{l}1 \\
\text { ( } 5 \text { of } 6 \text { chapters) }\end{array}$ & 177,845 & 35,569 \\
\hline 7. Programming & $\begin{array}{l}1 \\
\text { (15 of } 30 \text { chapters) }\end{array}$ & 180,024 & 12,002 \\
\hline 8. Operating Systems & $\begin{array}{l}1 \\
\text { (42 of } 51 \text { chapters) }\end{array}$ & 174,849 & 17,485 \\
\hline 9. Software and its engineering & $\begin{array}{l}1 \\
\text { (17 of } 25 \text { chapters) }\end{array}$ & 180,815 & 10,636 \\
\hline 10. Theory of computation & $\begin{array}{l}2 \\
(10 \text { of } 10) \text { and (2 of } 22) \\
\text { chapters }\end{array}$ & 174,179 & 13,398 \\
\hline Textbooks Total: & 12 (143 chapters) & $1,773,522$ & 15,835 \\
\hline
\end{tabular}

\subsection{CSAC2 size and balance}

The CSAC2 is the test corpus that was used to perform coverage tests during the analysis and evaluation of the CSAVL word list. Its structure mirrors that of the CSAC1 source corpus and the same methods which were described in the previous section (3.5) were used to balance token counts of the sub-corpora of the CSAC2 test corpus. For the 
smaller CSAC2 test corpus, a target size of 700,000 tokens was set. This is similar to the size of the test corpora that were used by both Coxhead and Minshall in evaluations of their respective word lists. The final CSAC2 test corpus contains chapters from 10 CS textbooks and 42 ACM journal articles. Table 3.8 outlines the structure of the 700,000 tokens of the CSAC2.

Table 3.8 Overall makeup of CSAC2

\begin{tabular}{lll}
\hline Text type & No. of texts & Tokens \\
\hline Textbooks & 10 & 356,679 \\
\hline ACM Journal Articles & 42 & 357,557 \\
\hline CSAC2 Total: & $\mathbf{5 2}$ & $\mathbf{7 1 4 , 2 3 6}$ \\
\hline
\end{tabular}

Each of the 10 ACM journal article sub-corpora contains from three to six ACM journal articles that total to approximately 35,000 tokens. The general structure of the journal article portion of CSAC2 is shown in Table 3.9. 
Table 3.9 Journal article makeup of CSAC2

\begin{tabular}{llll}
\hline Text type & No. of texts & Tokens & $\begin{array}{l}\text { Mean token } \\
\text { count per } \\
\text { article }\end{array}$ \\
\hline ACM Journal Articles & & & \\
\hline 1. Computer systems organization & 3 & 36,082 & 12,027 \\
\hline 2. Computing methodologies & 6 & 35,982 & 5,997 \\
\hline 3. Hardware & 5 & 36,802 & 7,360 \\
\hline 4. Human-centered computing & 5 & 35,415 & 7,083 \\
\hline 5. Information systems & 3 & 35,698 & 11,899 \\
\hline 6. Mathematics of computing & 5 & 35,218 & 7,044 \\
\hline 7. Networks & 3 & 35,032 & 11,677 \\
\hline 8. Security and privacy & 3 & 35,755 & 11,918 \\
\hline 9. Software and its engineering & 3 & 35,045 & 12,015 \\
\hline 10. Theory of computation & 6 & 35,528 & 5,921 \\
\hline Articles Total: & $\mathbf{4 2}$ & $\mathbf{3 5 7 , 5 5 7}$ & $\mathbf{1 2 , 3 8 7}$ \\
\hline & & & \\
\hline
\end{tabular}

Each of the 10 textbook sub-corpora contains from two to six chapter-length samplings of a single textbook. Each sub-corpus of the textbook portion of CSAC2 also contains close to 35,000 tokens. The general structure of the textbook portion of CSAC2 is shown in Table 3.10 . 
Table 3.10 Textbook makeup of CSAC2

\begin{tabular}{|c|c|c|c|}
\hline Text type & No. of texts & Tokens & $\begin{array}{l}\text { Mean } \\
\text { chapter token } \\
\text { count }\end{array}$ \\
\hline \multicolumn{4}{|l|}{ Textbooks } \\
\hline $\begin{array}{l}\text { 1. Computer systems } \\
\text { organization }\end{array}$ & $\begin{array}{l}1 \\
\text { (2 of } 8 \text { chapters) }\end{array}$ & 35,044 & 17,552 \\
\hline 2. Data Structures & $\begin{array}{l}1 \\
\text { (3 of } 28 \text { chapters) }\end{array}$ & 35,715 & 11,905 \\
\hline 3. Algorithms & $\begin{array}{l}1 \\
\text { (2 of } 13 \text { chapters) }\end{array}$ & 35,151 & 17,586 \\
\hline 4. Human-centered computing & $\begin{array}{l}1 \\
\text { (2 of } 10 \text { chapters) }\end{array}$ & 35,409 & 17,705 \\
\hline 5. Probability \& Statistics & $\begin{array}{l}1 \\
\text { ( } 2 \text { of } 16 \text { chapters) }\end{array}$ & 37,324 & 18,662 \\
\hline 6. Mathematics of computing & $\begin{array}{l}1 \\
\text { (4 of } 22 \text { chapters) }\end{array}$ & 36,306 & 9,077 \\
\hline 7. Programming & $\begin{array}{l}1 \\
\text { (5 of } 8 \text { chapters) }\end{array}$ & 35,772 & 7,154 \\
\hline 8. Operating Systems & $\begin{array}{l}1 \\
\text { (2 of } 14 \text { chapters) }\end{array}$ & 34,330 & 17,165 \\
\hline 9. Software and its engineering & $\begin{array}{l}1 \\
\text { (6 of } 13 \text { chapters) }\end{array}$ & 34,884 & 5,814 \\
\hline 10. Theory of computation & $\begin{array}{l}1 \\
\text { (2 of } 15) \text { chapters }\end{array}$ & 36,724 & 18,362 \\
\hline Textbooks Total: & 10 (30 chapters) & 356,679 & 11,889 \\
\hline
\end{tabular}

Since the CSAC2 was used to perform evaluative coverage tests of the final word list, all the texts included in the CSAC2 were from different authors than those sampled for the CSAC1. This was done in order to provide as fair a coverage test as possible for the evaluation of the word list and comparisons with other lists. All texts and specific token counts for CSAC2 are listed in the second half of Appendix E. 


\subsection{Data collection \& manual processing}

All of the journal articles and textbooks under consideration were available in electronic forms and were collected in PDF format. Textbooks were divided into separate chapters and preliminary word counts were made in order to make judgments about how many articles or chapters would be necessary to process in order to meet the target token counts for the two corpora. For textbooks and articles with large quantities of visual elements, mathematical formulae, or multi-column formatting, Adobe Acrobat was first used to drag and select elements for removal before converting to .docx format. For other files, PDFs were immediately converted to .docx format and MS Word was used for manual editing in order to take advantage of the wildcard search and replace functions.

Any element that was considered to be non-essential for the comprehension of a text was removed. This included reference lists, appendices, page titles, author's names, copyright information, publication names, acknowledgements sections, and numeric tabular data. Tables of linguistic data were converted to text delimited by tabs. Figure and table descriptions were kept, but repetitive labels that were placed outside of the main body of the text such as "Figure 1.2", "Example 3.2”, and "Lemma 1.4” were removed using wildcard search and replace expressions such as “Fig. ?.?” and “Table ?.?”. Because figure and table descriptions often interrupted sentences from the main text (creating fragmentation which could reduce part-of speech tagger accuracy), these descriptions were moved to a position after the nearest sentence ending. 
Because English reading comprehension can provide vital clues to understanding programming syntax, sections of programming code were not removed. As noted by Guo (2018), in a study of non-native English speakers learning computer programming, English is deeply intertwined with programming languages and found in identifiers, variables, functions, libraries, and class names. C functions such as "getch()” (get character) can be deciphered provided that the reader is familiar with the component words. Although it was not expected that abbreviations such as "getch" would meet the frequency criteria for the CSAVL, appearances of programming keywords such as “delete”, “switch”, “map”, and “null” within code samples contributed to the frequency counts which made them valid candidates for the CSAVL. In addition to naming conventions, instances of code were included since they were often found to be mixed with descriptive comment lines that provided explanations of the purposes of variables and functions, as seen in this example (where comment lines are prefaced with “//”):

// Sorts an array into ascending order.

// Precondition: anArray is an array of num integers and $1<=$ num $<=$ MAX_ARRAY, // where MAX_ARRAY is a global constant that specifies the maximum size of anArray . // Postcondition: anArray[0]<= anArray[1]<= ... <= anArray[num - 1];

// num is unchanged.

sort(anArray, num) 
Mathematical formulas composed of symbols and single- and multi-letter variables presented in isolation were removed, but those integrated into the main text (as in the following example) were kept at this stage in order to allow for human readability (for the concordance line corpus described at the end of this section) and also to prevent the additional loss of POS tagger accuracy.

Suppose $L_{0}$ is $n \times n$, obeys (1.2)-(1.3). Fix any $n \times n$ matrix I: of signs. Suppose that the support set $Q$ of $S_{0}$ is uniformly distributed among all sets of cardinality $m$, and that $\operatorname{sgn}\left(\left[S_{0}\right]_{i j}\right)=I_{i j}$ for all $(i, j) \in Q$. Then, there is a numerical constant c such that with probability at least $1-c n^{-10}$ (over the choice of support of $S_{0}$ ),

Titles, abstracts, and footnotes were kept intact. After the manual editing stage, .docx files were converted to plain .txt files in UTF-8 format. At this point in the processing, an additional backup copy of the corpus was made with the intention that this form of the corpus could be used to generate concordance lines of CS vocabulary usage for the development of data-driven learning (DDL) materials. The additional stages of processing would remove elements that may cause concordance lines to lose comprehensibility and important contextual clues for the purposes of human reading, so this backup was retained as a resource for language educators and students. 


\subsection{Automated processing}

Due to formatting decisions made by the publishers of the texts, all of the ACM journal articles and the majority of the textbook chapters contained a fairly large amount of end-of-line hyphenation. Many of the ACM articles contained 3-4 counts of end-ofline hyphenation per page, with some instances being split lexical items such as “difficult" and others being true hyphenations such as "low- rank". This resulted in the complication, as described in Mikheev (2005), where a regular expression could not be used to remove all instances of “- “ without concatenating true hyphenations and producing results such as “oneprocessor”.

In order to address this issue, a Perl script was found that provided a solution (Micher, 2012) by concatenating each hyphenated split and comparing it against a reference word list file in order to determine whether or not it is a true hyphenation. This script was modified for use on the CSAC texts and supplied with the top 25k frequency bands of the BNC and COCA (from Paul Nation’s Range program) as a dictionary reference text in order to verify instances of end-of-line hyphenation. Additional adjustments were made to the script so that it successfully ignored punctuation that was appended to words (e.g. “low-rank.”). In order for the script to function it was necessary to use regular expressions to first add a whitespace before every end of line ("`r|n”) character in all of the corpus files. The modified version of this Perl script is provided here in Appendix F. Though the script resolved the majority of hyphenation issues, it was unable to deal with hyphenations that were separated by line breaks ("low- In rank”) and 
low-frequency words not included in the dictionary reference, such as “virtual- ization”. These remaining instances were addressed through manual search and replace operations (“- “ replaced with “” or “-“).

It was also found that at some point during file format conversions, likely due to the variety of mathematical symbols appearing throughout the text, instances of "fl” (two characters) in words such as "fluid" had been automatically converted to "fl” (one character). This was resolved by replacing “fl, fi, ff, and ff”” with “fl, fi, ff, and ffi”. After this, all non-Unicode symbols and other undesired symbols were able to be removed with the regular expression “[^-a-z0-9A-Z_,.!?’;:()/—\[\]-'éls]”.

Brackets were kept at this stage since the IEEE standard is to enclose citations within them (e.g. “[Takahashi et al. 2002]”) and the brackets made these citations easily targetable by regular expressions. At this point, however, the citations could not be removed en masse due to an additional complication in which the ACM authors would sometimes use these citations as the object of a verb or preposition ("in [Cohen et al. 2010]”). In order to remove these citations without creating additional sentence fragmentation and reducing POS tagger accuracy, a program was developed in C++ to remove citations not followed by the words “in”, “see”, “at”, “of”, “following”, “as,” and “to". Citations preceded by one of those words were replaced by a dummy author name (“in [Cohen et al. 2010]” became “in Hernandez”) which would be filtered out in later stages along with other proper nouns. The source code for this program is provided here in Appendix G. After the citations had been removed, web page and FTP addresses were removed using this regular expression: 
$($ http $\mid$ ftp $\mid$ https $): / /\left(\left[\backslash w_{-}-\right]+\left(?:\left(?: \backslash .\left[\backslash w_{-}-\right]_{+}\right)+\right)\right)\left(\left[\backslash w_{.}, @ ?^{\wedge}=\% \&: / \sim+\#-\right]^{*}\left[\backslash w @ ?^{\wedge}=\% \& / \sim+\right.\right.$ $\#-])$ ?

Numbers and single letter mathematical variables were removed with the regular expression “ [b-h] | [j-z] | [B-H] | [J-Z] “ (which retained “A/a” and “I/i”). Multi-letter variables were manually removed later from the final word list. Although efforts were made to preserve hyphenation within the corpus, the removal of numbers resulted in the corpus containing hyphenated forms such as “real-time”, but also a large number of split forms such as “-dimensional” and “-bit”. In order to eliminate this inconsistency, all hyphens were removed from the corpus before tokenization.

\subsection{Word selection criteria}

Six word-selection criteria were established for the creation of the CSAVL, based on those set by Lei \& Liu (MAVL), who in turn drew their criteria from the work of Gardner \& Davies (AVL) and Coxhead (AWL). These six criteria were applied to the CSAC1 in order to choose the final set of lemmas that were included in the CSAVL. Section 3.11 provides details on the methods by which these criteria were applied to the data.

1. Minimum Frequency: This criterion requires that all items on the CSAVL occur with an overall minimum frequency of 100 times in the CSAC1 corpus. This was the minimum frequency set by Coxhead for the AWL, which was derived from a corpus of a similar size to the CSAC1 (3.5 million tokens). For the 3,532,486-token CSAC1, this equates to a normed frequency of 0.28 occurrences per ten thousand words. 
2. Frequency Ratio: The frequency of a lemma in CSAC1 must be $150 \%$ that of its frequency in a corpus of general English. This is based on Gardner and Davies' AVL criterion that was used to separate general high-frequency words from academic highfrequency words. In the case of the CSAVL, this allowed frequent CS words such as “memory” to be included, while excluding generally frequent words such as “the”. Following the example of Lei and Liu (2016), the non-academic portion of the BNC was used for this measurement. The normed BNC frequency (per ten thousand words) of each lemma was compared to its normed frequency in the CSAC1.

3. Range ratio: Lemmas must occur with at least $20 \%$ of their expected frequencies in at least half of the 20 sub-corpora. Expected frequency is calculated by dividing overall frequency of a lemma by the number of sub-corpora (20 in the case of the CSAC1). In creating the AVL, Gardner \& Davies required that lemmas occurred with $20 \%$ of expected frequency in 7 of their 9 disciplines (a range ratio of $78 \%$ ). For the AWL, Coxhead required that words appear in at least half of her sub-corpora (50\%). Lei \& Liu adopted Gardner \& Davies' minimum expected frequency requirement $(\geq 20 \%)$ and combined it with Coxhead's range requirement $(\geq 50 \%)$ since their corpus size was closer to the size of the AWL corpus. Lei \& Liu's decision was also adopted for the creation of the CSAVL in order to limit the inclusion of lexical items that are specific to a smaller range of the CS sub-disciplines.

4. Dispersion: Dispersion was used to ensure that lemmas were evenly distributed throughout the corpus. Following Gardner and Davies (2014) and Lei and Liu (2016), Juilland's D was selected as the measure of dispersion. All lemmas must have a 
minimum dispersion value of 0.3 . The ideal target threshold is arbitrary and is a topic of debate (Gries, 2019). Gardner and Davies set this value at 0.8 for the creation of the Academic Vocabulary List (AVL), whereas Lei and Liu chose the value of 0.5 when creating the Medical Academic Vocabulary List (MAVL) from a much smaller corpus than the 120-million-word COCA academic corpus used by Gardner and Davies. The Juilland's D threshold of 0.3 was chosen by Oakes and Farrow (2007) in their examination of the most typical lexical items in a set of seven corpora which represented English usage in seven different countries. Oakes and Farrow noted that the choice of this threshold figure is arbitrary, and arrived at the chosen value of 0.3 after examining their results and making subjective evaluations of the degree that the resulting words could be considered typical of each corpus.

For this project (the CSAVL), Lei and Liu's dispersion threshold level of 0.5 was initially chosen because the CSAC1 was similar in size to the corpora that were used by Lei and Liu to generate the MAVL (2.7 million words). However, the 0.5 threshold was found to be too restrictive a choice for a corpus including a large amount of technical vocabulary. The 0.5 threshold resulted in a shorter word list than that generated by Lei and Liu, and only achieved a limited amount of coverage in a CS corpus. After experimenting with a variety of values, performing coverage tests, and examining the list contents for degree of technicality (analysis of technicality is described in Section 4.7 and discussed again in Section 5.3), a final dispersion threshold of 0.3 was chosen, matching the value selected by Oakes and Farrow (2007). 
5. Discipline measure: A lemma should occur no more than 3 times its expected frequency in any more than three of the sub-corpora. This also follows Lei \& Liu's modifications of Gardner \& Davies’ criterion. This measure prevents lemmas from clustering in a section of the corpus while being infrequent in the remaining sections, which prevents technical items that are primarily used within a limited number of subdisciplines from being included in the list.

6. Special meaning criterion for general high-frequency words: Lemmas identified as being general high-frequency words must also appear in a technical dictionary to be included in the CSAVL. This step ensures that these general high-frequency words take on special meanings within a CS context. General high-frequency words were identified by comparing CSAVL candidate lemmas (separated by part of speech) with Brezina \& Gablasova’s (2015) New General Service List (new-GSL) and finding matching items. Those items then needed to also be found in the Oxford Dictionary of Computer Science (ODOCS) (Butterfield et al., 2016) in order to remain among the CSAVL candidates.

\subsection{Tokenization and part of speech tagging}

In order to apply these criteria and create the word list, the corpora texts were then tokenized, tagged with parts of speech, and lemmatized. For this purpose, Lancsbox (Brezina et al., 2015, 2018), which uses the TreeTagger POS tagset, was used to perform all three tasks. The Lancsbox tools allowed for calculations of frequency, relative 
frequency (per 10k words), and dispersion to be made, all of which were necessary for applying the criteria listed in the previous section. Lists of these statistics were made for the combined CSAC1 and for each of the 20 sub-corpora of the CSAC1.

Since criterion two required a comparison with the non-academic portions of the British National Corpus (BNC), Lancsbox was also used to generate a list of BNC lemmas (separated by part of speech) with their relative frequencies. The BNC, in its publicly available XML format, did not have filenames which described their contents. In order to accomplish the task of removing the academic portions, a special function of WordSmith Tools (Scott, 2016) was used to restore content labels to each of the BNC files. After the academic files had been removed, Lancsbox could then be used to generate the desired statistical output lists. All of the resulting lemma lists (separated by

part of speech) were exported from Lancsbox as tab-delimited text files which were then used as the input data in the analysis stage.

\subsection{Extracting CSAVL candidate lemmas}

After the initial statistics had been generated in Lancsbox, the files were converted from tab-delimited text files to Excel format. In Excel, each statistic was sorted from highest to lowest value and items that were below a frequency of 100 or a dispersion of 0.3 were removed. This step applied criteria one and four (minimum frequency and 
dispersion), reducing the initial 46,977 lemmas found in CSAC1 to a list of 2,721 candidate lemmas.

A set of programs was developed in $\mathrm{C}++$ in order to apply the remaining criteria. The programs read the items listed in the candidate files, evaluated whether or not the desired criteria were met, and then generated text files listing items that met the desired criterion as well those which were rejected. The file listing successful candidates was then used as the input file for the next program in the sequence. The first program in the sequence compared the CSAC1 relative frequency list to that of the BNC, eliminating items that occurred at less than 150\% of their frequency in the BNC (applying Criterion 2). The 970 lemmas removed during this stage included many general high-frequency items such as 'the_other', 'be_v', and ‘of_con', and left a remaining list of 1,751 lemmas.

The second program compared the combined CSAC1 to each of its sub-corpora, applying criteria three and five (range ratio and discipline measure) by removing likely discipline-specific and technical terms. A total of 342 lemmas were removed in this stage, including “granularity_n”, “surveillance_n”, and “accelerometer_n”, and leaving 1,409 remaining lemmas.

The final program applied criterion six (special meaning criterion for general highfrequency words) by searching for the remaining lemmas in the new-GSL and ODOCS and removing any new-GSL lemmas that did not have CS-specific meanings. This phase required that specially formatted versions of both the new-GSL and ODOCS be created (one word per line with related values separated by tab spacing). Additionally, the newGSL (as provided in a supplemental materials link in the 2015 Brezina and Gablasova 
article) part-of-speech tags differed somewhat from the part-of-speech groupings used by Lancsbox, so the new-GSL list was altered using regex replacement so that it could be better compared to the Lancsbox-generated data. "mod” (modal) was replaced with "v" (verb), “avp” (adverbial particle) was replaced with “adv” (adverb), “x” (other) was replaced with “other”, “abr” (abbreviation) was replace with “other”, and “t” (infinitive to) was replaced with “other”.

Because the ODOCS does not specify parts of speech for its entries, part of speech was not considered when comparing the CSAC1 to ODOCS entries. This program also flagged lemmas which were found in the new-GSL and ODOCS when outputting the final list for later reference. In this third phase, 458 lemmas were removed, leaving a remain list of 951. The entire process of candidate lemma extraction is depicted in flowchart form in Figure 3.2.

Once all six criteria had been applied and the remaining candidate items for the CSAVL had been determined, manual edits were made to the remaining items. Multiletter variables and numerals (“dt_n”, “iii_n”), initialisms and acronyms (“ip_n”, “url_n”), and proper nouns and adjectives (“google_n”, “gaussian_adj”), and prefixes (“multi_n”, “quad_n”) were removed from the word list. Items that appeared on the newGSL in alternate spellings (“colour_n”) were also removed. At this point, it was found that TreeTagger had incorrectly tagged many of the verbs used in the imperative mood in textbook exercises (“assume_n”, “compare_n”, “describe_n”). These POS labels were manually corrected in the Lancsbox output files and the entire extraction sequence was restarted using these new adjusted labels. After these manual adjustments and edits 
were made and the list was re-processed, a total of 876 lemmas remained on the preliminary version of the CSAVL. An additional 28 lemmas were also included after early experiments combining the list with the new-GSL (detailed in Section 3.13), resulting in a total of 904 lemmas in the final version of the CSAVL. 


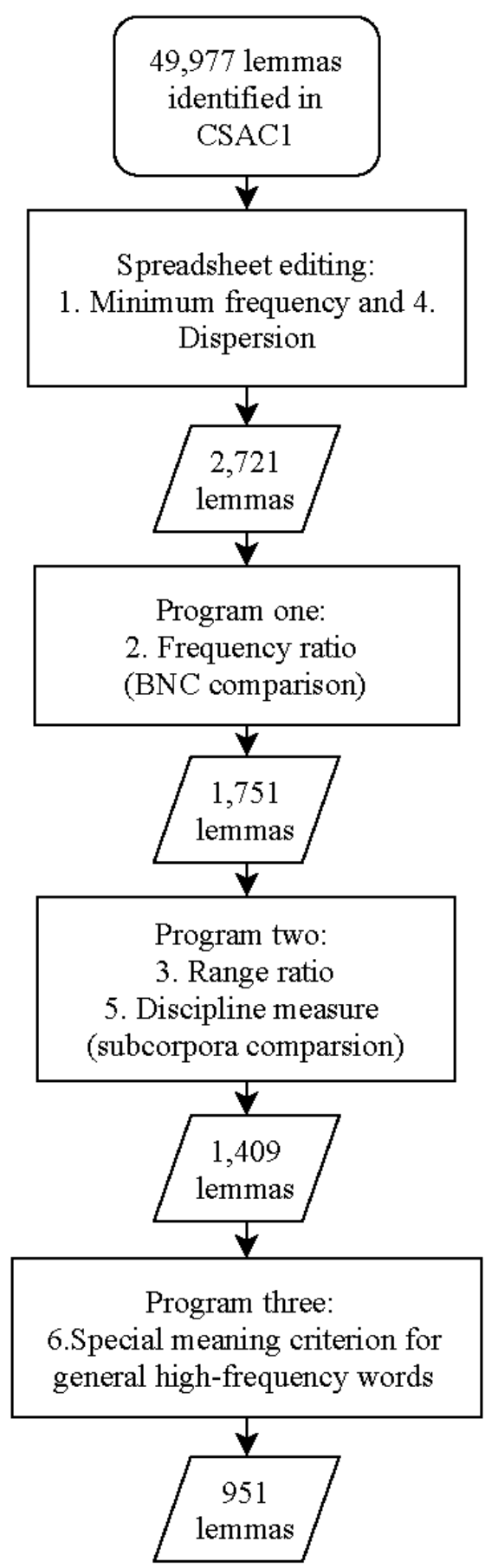

Figure 3.2 Lemma extraction flowchart 


\subsection{Extracting CSAVL-S candidate lemmas}

In addition to the CSAVL academic list, a supplement word list, the CSAVL-S, was created in an attempt to reach the 95\% lexical coverage threshold suggested by Laufer (1989, 2010) for a minimal level of reading comprehension. While the CSAVL was intended to include the most frequently used items of CS academic vocabulary, the CSAVL-S was designed to include technical words that occurred more frequently within CS subdisciplines than they did relative to the discipline as a whole. Nation (2016) described technical vocabulary as occurring only in a subject area or in that subject area with a narrowed technical meaning, and also stated that most technical vocabulary will occur more often in the subject area than outside of it (p. 146). Given this description, sub-disciplinary technical items would likely be clustered in certain sub-corpora of the CSAC1.

A list of candidate lemmas for the CSAVL-S was extracted in the following manner. First, since the CSAVL-S was a supplemental list, items already on the CSAVL were excluded. Next, the criteria which limited the inclusion of sub-disciplinary technical items were either modified or removed: criteria three (range ratio), four (dispersion), and five (discipline measure) were removed, and criterion one (minimum frequency) was lowered from 100 to 60 .

Using the programs described above to only apply criteria two (frequency ratio) and six (special meaning), a preliminary list of 667 CSAVL-S candidate lemmas was produced. In addition to these 667 lemmas, 35 lemmas were added after experiments 
which combined the list with the new-GSL (detailed in Section 3.13). The final version of the CSAVL-S includes a total of 702 lemmas.

\subsection{Additions to the CSAVL and CSAVL-S}

In order to address the second research question regarding the combination of the CSAVL/CSAVL-S lists with a general high-frequency word list, a three-list combination of the new-GSL, CSAVL, and CSAVL-S was made with all overlapping lemmas removed. Through the process of creating and testing this combined word list, a number of lemmas were identified as candidates for expansion of the CSAVL and CSAVL-S. The new-GSL was the first general list chosen for this experiment since, like the CSAVL, it was in organized in a standard lemma form and tagged with parts of speech. Browne's NGSL, also was found to be considerably less efficient than the new-GSL after making comparisons at the lemma level. Additionally, a relationship between the new-GSL and CSAVL had already been established when the new-GSL was used as the general list which limited the inclusion of general frequency items without specific meanings in the CSAVL (Criterion six).

An additional piece of software was developed which removed the overlapping contents of the new-GSL, CSAVL, and CSAVL-S. A program was also developed to perform lemma-based coverage checks by comparing the word lists to the statistical lemma count information generated by Lancsbox. In addition to the coverage statistic, the 
program also produced a list of items not found by the lists which guided the identification of possible expansion lemmas for the CSAVL/CSAVL-S.

Early coverage checks of the combined new-GSL/CSAVL/CSAVL-S on the CSAC2 test corpus showed that numerous lemmas that had been removed by the BNC comparison stage had CS-specific meanings, such as “gate_n”, “pipe_n”, and “printer_n”. These items with CS-specific meanings had been removed by the BNC criterion which had been intended to target general frequency items. For the majority of these lemmas, the Oxford Dictionary of Computer Science (ODOCS) was used to identify items with CS-specific meanings. Several additional items were included based upon my own knowledge of CS. A total of 28 of these lemmas (which were found to satisfy all five other list inclusion criteria) were added to the CSAVL, resulting in a total of 904 lemmas. Another 35 lemmas which were similarly found to have CS-specific meanings such as “crack_v” and "serial_adj” and also met the criteria for inclusion in the CSAVL-S. The inclusion of these items brought the technical list to a total of 702 lemmas.

\subsection{Removing undesired content from the CSAC2}

Before final coverage tests could be performed of the CSAVL and CSAVL-S on the CSAC2 test corpus, the CSAC2 was examined for items which would be ignored in the tests. Numbers and all non-alphabetic characters were removed from the CSAC2 using regular expressions. 
As Nation (2016) argued, proper nouns are not necessarily unique to a single language, carry little meaning beyond a specific referent, and are usually omitted in word counts related to vocabulary lists. Vocabulary list developers (Coxhead, 2000; Konstantakis, 2007; Minshall, 2013) have also omitted proper nouns, acronyms, and abbreviations from their corpus token counts due to these terms often being recognizable across languages (“PC”) and placing a low learning burden on a learner (Konstantakis, 2007). Following this reasoning, proper nouns and adjectives (“Microsoft”, “Bayesian”), acronyms (“BIOS”), and initialisms (“CPU”) were removed from the corpus. Affixes that had been separated by hyphen removal (“multi”), and abbreviations (“stdlib”) were also removed. This was accomplished using files from Paul Nation's Range program (Nation \& Heatley, 2002), basewrd31.txt (a collection of proper nouns), basewrd34.txt (a collection of acronyms and initialisms), and the top 25,000 frequency band items of the BNC/COCA that are included with the program.

AntWordProfiler (Anthony, 2008) was used to compare the CSAC2 to the following combination of six word lists: Nation's BNC/COCA 25k, basewrd31, basewrd34, the NGSL (Browne, 2014), the CSAVL, and the CSAVL-S. The list of items not found by this combination was then manually examined for the remaining proper nouns and other undesired elements listed above. These items were then combined with Nation's basewrd31 and basewrd34 files to produce a CSAC2 master filtering list. A program was written in order to remove all items listed on the filtering list from the CSAC2 before coverage checks were performed. These undesired items were found to comprise a total of 3.99\% of the CSAC2 (shown in Table 3.11). 
Table 3.11 Undesired content in CSAC2

\begin{tabular}{ll}
\hline basewrd31.txt & $1.01 \%$ \\
\hline basewrd34.txt & $0.57 \%$ \\
other undesired & $2.41 \%$ \\
total & $3.99 \%$ \\
\hline
\end{tabular}

This 3.99\% figure was similar to the percentages of similar content found in the corpus used to generate the CSWL (Minshall, 2013) (3.24\%) and the percentage of proper nouns and other "non-word" content found in the two corpora used for the MAVL (Lei and Liu, 2016) (3.67\% and 4.65\%).

After using Lancsbox to identify lemmas in the CSAC2, some inaccuracies in the POS-tagging process were found. Verbs used in the imperative mood were tagged as nouns, and neologisms which were not found in the lemmatizer's dictionary were incorrectly grouped (inflected forms such as “workflow_n” and “workflows_n” were separately listed). These lemmas were manually corrected, and each correction was verified by examining concordance lines in the CSAC2.

\subsection{Comparing structurally different word lists}

In order to evaluate the CSAVL and CSAVL-S word lists, comparisons were made with a number of other academic and general high-frequency word lists. While the CSAVL and CSAVL-S are lemma-based lists, some of the comparison lists were based on different units of counting, such as the word family. As noted in Section 2.6, for these types of comparisons to be as fair as possible, Nation (2016) recommends that 
conversions of units are performed (e.g. word families are converted to lemmas). This section (3.15) describes the methodology that was used to perform these unit conversions and provides additional information about the structural differences between four of the units that have been used to count words: the type, the word family, the lemma, and the modified lemma (used by Browne for the 2014 New General Service List).

Figure 3.3 provides an example that will be used to illustrate the structural differences between these different units of word counting. These first of these counting units, the type, is a word form with a different spelling. When counting Figure 3.3 by types (different spellings) a total of six types can be found (“bank”, "banks”, "banked”, “banking”, “banker”, “bankers”). The word family counting unit contains all inflectional and derivational forms up to level 6 of Bauer and Nation’s (1993) rating system (as described in Section 2.3). Figure 3.3 contains only a single word family headword (“bank”). The lemma counting unit contains all inflectional forms within a single part of speech. When counting Figure 3.3 by lemmas, a total of five lemmas can be found (“bank_v”, “banked_adj”, “bank_n”, “banking_n”, “banker_n”). The modified lemma counting unit contains all inflectional forms, but ignores parts of speech. Figure 3.3 contains two modified lemmas (“bank” and "banker”). Nation (2016) suggests there is an approximate ratio of 1:3:6 when converting between word families, lemmas, and types. This ratio is an approximation, so when converting between word counting units, results may vary. For the case of the set of words making up Figure 3.3, there is a ratio of 1:5:6 when converting between word families, lemmas, and types. 


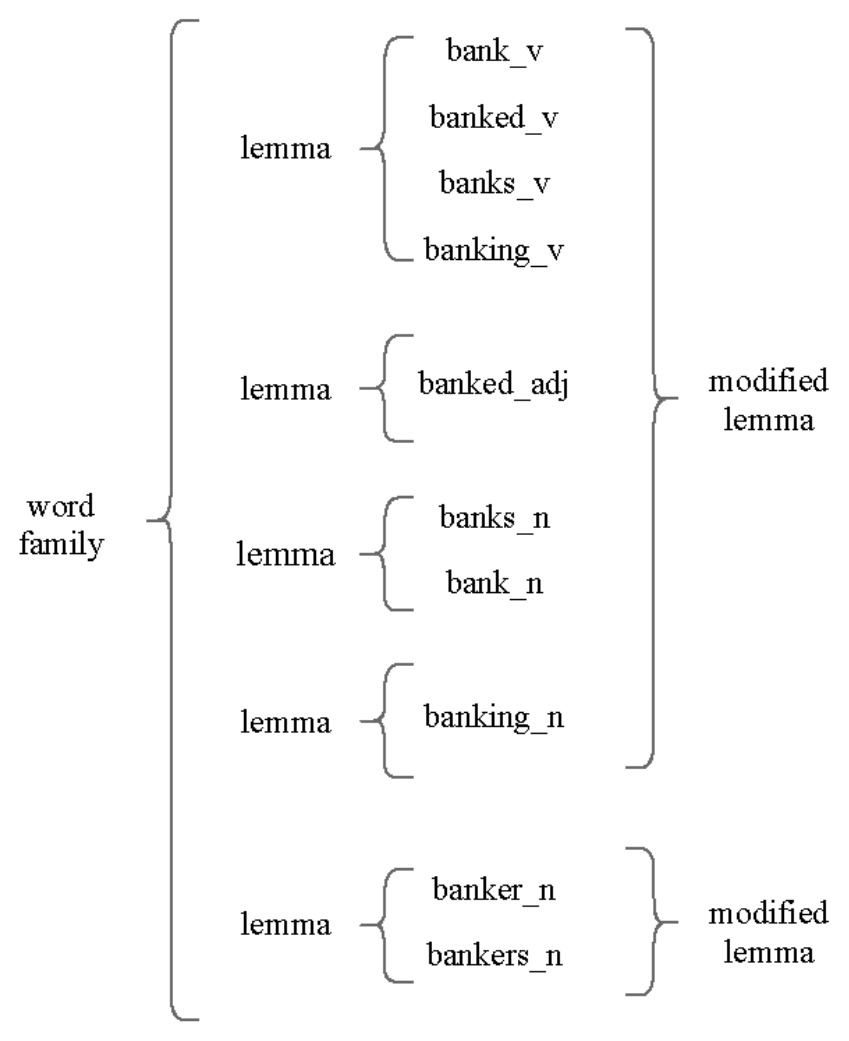

Figure 3.3 Word unit conversion

Nation’s approximate conversion ratio of 1:3:6 between word families, lemmas, and types means that a word family-based list such as the GSL contains approximately three times as many lemmas as it does word families. The version of the GSL that is commonly distributed with software such as Range (Nation \& Heatley, 2002) and AntWordProfiler (Anthony, 2008) contains 2,000 word family headwords, and after I converted this list to lemma form, it contained approximately 5,338 lemmas (shown in Table 3.12). This figure was determined by using Lancsbox (Brezina et al., 2015, 2018) to analyze the GSL in type form, and is likely to be fewer than the actual number of lemmas contained in the GSL since the widely distributed version which was analyzed does not include all 
possible derivations up to level 6 of Bauer and Nation’s (1993) rating system (derivations such as "bankable” are absent from this version). The figure of 2,000 "words” could lead word list users to the impression that the GSL is the "shortest" general high-frequency word list. However, when compared at the lemma level, a general service list such as Brezina and Gablasova's 2,495-lemma new-GSL is less than half the size of West's 5,068-lemma GSL.

To allow for the comparison of the lemma-based CSAVL and CSAVL-S with word family-based lists, type and lemma counts for the word family lists (the GSL, AWL, and CSWL) were produced. To produce these counts, each list was converted from word family headwords to type form using the "Familizer + Lemmatizer" available on the Lextutor (Cobb, n.d.) website. The type forms of the lists were then analyzed with Lancsbox to determine the number of lemmas. As Table 3.12 shows, the CSAVL is also less than half the size of the AWL when comparing by lemmas, and the CSAVL-S is $57 \%$ of the size of the comparable CSWL technical word list. 
Table 3.12 Word list sizes

\begin{tabular}{lrrrr}
\hline word list & types & lemmas & $\begin{array}{r}\text { modified } \\
\text { lemmas }\end{array}$ & families \\
\hline GSL (West, 1953) & 7,822 & 5,338 & $3,623^{*}$ & $\mathbf{2 , 0 0 0}$ \\
AWL (Coxhead, 2000) & 3,082 & 1,926 & & $\mathbf{5 7 0}$ \\
CSWL (Minshall, 2013) & 1,919 & 1,225 & & $\mathbf{4 3 2}$ \\
NGSL (Browne, 2014) & 8,480 & 4,770 & $\mathbf{2 , 8 0 1}$ & \\
new-GSL(Brezina \& Gablasova, 2015) & 5,115 & $\mathbf{2 , 4 9 5}$ & & \\
CSAVL & 1,853 & $\mathbf{9 0 4}$ & & \\
CSAVL-S & 1,398 & $\mathbf{7 0 2}$ & & \\
\hline
\end{tabular}

* Value stated in Browne, 2014

Bolded values indicated the original unit of list organization.

In addition to the comparisons made of the CSAVL and CSAVL-S with word family-based lists such as the AWL and CSWL, comparisons were also made with Browne’s (2014) New General Service List (NGSL), a word list which was based on the modified lemma grouping. As Figure 3.3 illustrates, the modified lemma ignores parts of speech and instead forms word groups based on inflection. The forms "bank”, "banks”, “banked”, and "banking” are grouped together in a single modified lemma, while the derivations "banker” and "bankers” are grouped in another. In order to convert the modified lemma-based NGSL to lemma form, I used the Lancsbox software to analyze the type version of the NGSL that is available as an AntWordProfiler (Anthony, 2008) supplement. It was found that though the NGSL contains only 2,801 modified lemmas, those modified lemmas convert to a total of 4,770 lemmas. When compared at the lemma level, the 2,495-lemma new-GSL (Brezina \& Gablasova, 2015), which provides comparable coverage to Browne’s 4,770-lemma NGSL, again appears to be the more efficient list. 
A list in word family form can be converted to lemma form without distorting the intended contents of the list since the word family is assumed to include all parts of speech for each headword. The word family headword "bank" is assumed to include “bank_v”, “bank_n”, “banking_n”, “banked_adj”, and “banker_n”. However, when converting in the opposite direction (from lemma to word family), a different relationship is found. If one converts "bank_v" to "bank" (word family headword), then new information has been added (“banker”, "bankers”) which no longer reflects the contents of the original lemma-based word list. This results in unfair coverage comparisons between lists of different structure (as noted by Gardner and Davies, 2014). For this reason, the word lists shown in Table 3.12 are not compared at the word family level, and all further comparisons are made at the lemma or type level.

\subsection{Summary of methodology}

In first half of the methodology chapter, I described the steps involved in the creation of the CSAC1 and CSAC2 corpora. This description included the corpora design (Section 3.1), the types of texts that were included (Section 3.2), the criteria for article and textbook selection (Sections 3.3 and 3.4), the balance of sub-corpora (Sections 3.5 and 3.6), and the data collection and processing (Sections 3.7 and 3.8).

In the second half of the chapter, I detailed the process of developing the CSAVL and CSAVL-S word lists. I outlined the criteria for inclusion in the CSAVL (Section 3.9), 
the tokenization and tagging process (Section 3.10), the extraction of lemmas (Sections 3.11 and 3.12), the revisions to the lists (Section 3.13), and the preparation of the CSAC2 test corpus for coverage testing (Section 3.14). This was followed by a description of the methods used to compare lists with different units of counting (Section 3.15). The next chapter presents the results of the comparisons that were made between the CSAVL/CSAVL-S and other word lists with similar purposes. 


\section{Chapter 4: $\quad$ Results}

Following Nation's (2016) recommendation that word lists are compared with other lists that have a similar purpose, the first sections of this chapter (Sections 4.1 to 4.3 ) are coverage test comparisons of word lists with similar intended uses to that of the Computer Science Academic Vocabulary List (CSAVL). These tests were all performed using the Computer Science Academic Corpus 2 (CSAC2), the test corpus that was created for the purposes of evaluating the CSAVL. The first section, Section 4.1, begins the chapter with a comparison of the CSAVL and several other academic word lists. This is followed by a comparison of the Computer Science Academic Vocabulary Supplemental List (CSAVL-S) with Minshall’s (2013) Computer Science Word List (CSWL) in Section 4.2. The chapter is continued in Section 4.3 with a comparison of several general high-frequency word lists that were combined with the CSAVL and CSAVL-S in an attempt to reach the target lexical threshold suggested by Laufer (1989) for a minimal level of reading comprehension.

In the following sections, coverage tests are performed on other corpora and the contents of the CSAVL and CSAVL-S are compared with those of other word lists. Section 4.4 reports the results of the Computer Science Academic Vocabulary List (CSAVL) coverage tests across corpora that were chosen to represent general, academic, and academic computer science English usage. Section 4.5 presents the results of CSAVL content comparisons with the entries of technical dictionaries in order to evaluate the degree of the list's technicality. Section 4.6 contains a comparison of the contents of the 
CSAVL with those of the General Service List (GSL) (West, 1953), Academic Word List (AWL) (Coxhead, 2000), and Computer Science Word List (CSWL) (Minshall, 2013). After identifying the overlapping contents between the GSL, AWL, and CSWL word lists and the New General Service List (new-GSL) (Brezina \& Gablasova, 2015), CSAVL, and CSAVL-S, results of coverage tests of the overlapping and non-overlapping contents are presented in Section 4.7. The chapter is concluded in Section 4.8 with a summary of the results.

\subsection{Academic word list coverage comparisons}

The final version of the Computer Science Academic Vocabulary List (CSAVL) contains a total of 904 lemmas and is listed in Appendix A. The CSAVL supplemental list (the CSAVL-S) contains a total of 702 lemmas and is listed in Appendix B. The lemmas of the CSAVL and CSAVL-S are ranked in frequency order, with the frequency rank number displayed to the left of each entry. Lemmas that have CS-specific meanings have been flagged with “*” to indicate items with matching entries in the Oxford Dictionary of Computer Science (ODOCS). Lemmas with mathematics-specific meanings are marked with “\#” to indicate matching entries in the Oxford Concise Dictionary of Mathematics (ODOM).

Using these final versions of the CSAVL and the CSAVL-S, I performed coverage checks on the Computer Science Academic Corpus 2 (CSAC2), the test corpus that was 
created for the purposes of evaluating the CSAVL. A coverage test determines the percentage of tokens in a given corpus that correspond with the items on a word list.

Coverage tests were performed for two reasons. First, to examine list representativeness: by determining the number of tokens of an academic computer science corpus that is covered by a word list, we can gain some indication of the word list's representativeness of the target population of texts (i.e. to what degree do the contents of a word list represent the contents of frequently-used academic CS texts in American universities in 2019?). Second, to determine list efficiency: by comparing both the coverage percentage and the size of the word list, we can get an indication of the cost/benefit relationship of the list for the target group of users (English language learners studying CS at American universities). A word list that achieves the same amount of coverage as another list, but does so with fewer words, could be described as a more efficient list because it will likely result in a shorter time investment for learners.

Nation (2016) recommends that when comparing word lists, the lists being compared have a similar purpose. For the evaluation of the CSAVL, the most ideal comparison would be one made between the CSAVL and another CS-specific academic word list. However, since the only CS-specific word list that was available, The Computer Science Word List (CSWL) (Minshall, 2013), is a supplemental list to the Academic Word List (AWL) (Coxhead, 2000) and does not attempt to independently describe academic CS vocabulary, the CSAVL will be instead compared to general academic word lists when making single-list comparisons. The general academic word lists that are compared with the CSAVL in this section, The Academic Word List (AWL) (Coxhead, 2000) and the 
Academic Vocabulary list (AVL) (Gardner \& Davies, 2014), might be viewed as the alternative choices for educators and learners who hope to develop reading comprehension of academic computer science texts. For this reason, they were chosen for the comparison in this section.

Though the word lists that are compared in this section all describe academic vocabulary, these lists are not of the same size; the AVL contains 3,015 lemmas, the AWL contains 1,926 lemmas, and the CSAVL contains 904 lemmas. Because of these size differences, normed coverage values (coverage per 100 lemmas and coverage per 100 types) are given to provide an accurate basis of comparison other than the total coverage of the CS corpus. Because the lists being compared in this section describe a similar range of vocabulary, but are of notably different sizes, normed coverage will be used a metric to determine each list's efficiency in covering a corpus of academic CS texts.

The coverage tests performed in this analysis were of two varieties: part of speech (POS)-based and type-based. In a POS-based coverage test, a POS-tagged word list (with items such as "run_v") is compared with a POS-tagged corpus. A POS-based test is necessary in order to provide accurate coverage measures of a lemma-based word list. In a POS-based coverage test, if a corpus were to contain three instances of "run_n”, and a word list only contained "run_v", there would be no coverage (no match). Type-based coverage tests, which are used to perform coverage tests on word family-based lists and modified lemma lists, do not examine parts of speech, and only match types (spellings). In a type-based coverage test, if the word list contains "run” and the corpus contains 
"run”, there is a match, regardless of part of speech. To provide coverage values that are as accurate as possible, all word lists were evaluated based on their unit of counting: all word lists in lemma form were evaluated using POS-based tests, and all word lists in word family or modified lemma forms were evaluated using type-based tests.

POS-based coverage tests were performed using custom software that I developed for this task. This program compared the POS-tagged word lists to the CSAC2 lemma count information generated by Lancsbox (Brezina et al., 2015, 2018) in order to produce a coverage percentage value. In addition to POS-based coverage tests, type-based coverage tests were performed using AntWordProfiler (Anthony, 2008) for the lists that are distributed in type form (word family and modified lemma-based lists). The typebased tests were performed on an untagged version of the CSAC2. As previously described in Section 3.14, the versions of the CSAC2 that were used in both types of coverage tests had been stripped of undesired content (3.99\% of the tokens had been removed).

Type counts of lemma-based lists are reported in this chapter to provide another counting unit for the comparison of word list sizes. These type counts were determined through the following two-step process: First, all lemmas in the word list were expanded to all possible inflectional forms using the "Familizer + Lemmatizer” (Cobb, n.d.) website; second, all inflectional forms that were not originally part of that lemma's POS were removed. For example, if a lemma list were to contain only the item “drink_n”, after automatically converting the list to all inflectional forms using the Familizer + Lemmatizer (software which does not consider part-of-speech information), the result 
would be “drink”, “drinks”, “drinking”, “drank”, and “drunk”. Because the original lemma list only contained “drink_n” as noun, then the results “drinking”, “drank”, and “drunk” would have to be removed in order to accurately represent the noun (“drink_n”) that appeared in the original lemma list.

Table 4.1 shows the results of the coverage tests performed on the CSAC2 using the CSAVL and two of the word lists which focus on academic vocabulary: the lemma-based Academic Vocabulary List (AVL) (Gardner \& Davies, 2014) and the word family-based Academic Word List (AWL) (Coxhead, 2000). The largest of the three lists, Gardner and Davies’ AVL, covered $18.64 \%$ of the CSAC2 corpus. In comparison, the CSAVL, which is less than one third of the size of the AVL, covered $16.06 \%$ of the corpus. Though the AVL exceeded the total coverage of the CSAVL by $2.58 \%$, the large size of the AVL diminishes its efficiency for word list users. When the normed coverage per 100 lemmas values are compared to account for the difference in size, the CSAVL covered $1.78 \%$ of the corpus per 100 lemmas, nearly tripling the efficiency the AVL's $0.62 \%$ coverage per 100 lemmas. In addition to the AVL's limitation in terms of efficiency, the AVL is also limited by its status as a standalone academic vocabulary list. The CSAVL can be supplemented by the CSAVL-S to provide additional coverage of an academic CS corpus while remaining more efficient than the AVL (as shown in Table 4.2).

When comparing the CSAVL with Coxhead's AWL, the CSAVL (which covered $16.06 \%$ of the corpus) provided higher total coverage than the AWL (12.2\%) while containing less than half the number of lemmas. When examining the normed coverage values, it can be seen that the CSAVL's normed coverage of 1.78\% per 100 lemmas 
nearly tripled the $0.63 \%$ coverage per 100 lemmas provided by Coxhead's AWL.

Coxhead's strict exclusion of all GSL content (some of the most highly-frequent words in the English language) from the AWL is a likely factor for its relative low coverage of the CS corpus in this set of tests. Other factors that may have resulted in the difference in coverage between the CSAVL and AWL are discussed in Section 4.6, where the specific contents of AWL and CSAVL are examined in detail.

Table 4.1 CSAC2 coverage of the AVL, AWL, and CSAVL

\begin{tabular}{llcccccc}
\hline word list & $\begin{array}{l}\text { test } \\
\text { format }\end{array}$ & types & lemmas & families & $\begin{array}{r}\text { CSAC2 } \\
\text { coverage }\end{array}$ & $\begin{array}{r}\text { coverage } \\
\text { per 100 } \\
\text { types }\end{array}$ & $\begin{array}{r}\text { coverage } \\
\text { per 100 } \\
\text { lemmas }\end{array}$ \\
& & & & & & & \\
\hline $\begin{array}{l}\text { AVL (Gardner } \\
\text { \& Davies, }\end{array}$ & POS & 5,340 & 3,015 & & $18.64 \%$ & $0.35 \%$ & $0.62 \%$ \\
$\begin{array}{l}\text { 2014) } \\
\text { AWL } \\
\text { (Coxhead, }\end{array}$ & & & & & & \\
$\begin{array}{l}\text { 2000) } \\
\text { CSAVL }\end{array}$ & type & 3,082 & 1,926 & 570 & $12.20 \%$ & $0.40 \%$ & $0.63 \%$ \\
& & & & & & & \\
\end{tabular}

\subsection{Supplemental CS word list coverage comparisons}

This section provides a comparison between two supplemental academic computer science vocabulary lists: the CSAVL-S and the Computer Science Word List (CSWL) (Minshall, 2013). In this section, I demonstrate that the CSAVL-S provides similar normed coverage of an academic CS corpus to the CSWL and that the CSAVL\CSAVL- 
S combination provides more than double the normed coverage of the AWLICSWL combination in a corpus of academic CS texts.

Since the CSWL is a supplemental list to the AWL, and does not attempt to independently describe the vocabulary of academic CS, it was not directly compared to the main CSAVL word list. However, a comparison is also made of the combinations of the supplemental lists with their base lists; the combination of the CSWL and AWL is compared with the combination of the CSAVL and CSAVL-S.

The first two rows of Table 4.2 provide the results of the coverage tests that were performed using the CSAVL-S and CSWL word lists on the Computer Science Academic Corpus 2 (CSAC2). The larger of the two lists, Minshall’s CSWL, provided a total coverage of 5.57\%, whereas the smaller list, the CSAVL-S, provided a total coverage of 3.84\%. When examining the normed coverage values, it can be seen that the CSAVL-S provides slightly higher coverage per 100 lemmas (0.55\% versus the CSWL’s 0.45\%) and equal coverage per 100 types. The similarity in the normed coverage values between the lists suggests that these lists may also be similar in their level of efficiency for users. Given that the normed coverage is similar between the lists, the size difference of the lists $(1,225$ lemmas in the CSWL compared to 702 lemmas in the CSAVL-S) is a likely to be the primary factor in the difference in total coverage. The relationship between the CSWL, CSAVL, and CSAVL-S is further analyzed in Section 4.6 where some of the specific contents of these lists are examined.

The third and fourth rows of Table 4.2 also list the results of the coverage tests that were performed using combinations of the supplemental lists and their base lists. The 
combination of the CSAVL/CSAVL-S covered 19.9\% of the CSAC2, slightly higher than the $17.26 \%$ covered by the AWL/CSWL combination. The CSAVL/CSAVL-S

combination, being smaller in size while providing more coverage of a CS corpus, was found to be the more efficient combination in this comparison. The combined CSAVL/CSAVL-S combination totaled to 1,606 lemmas, nearly half of the total size of the 3,151-lemma AWL/CSWL combination. When examining the normed per 100 lemma coverage of the combinations, the CSAVL/CSAVL-S (1.24\%) more than doubled the normed coverage of the AWL/CSWL (0.55\%).

Table 4.2 CSAC2 coverage of the AWL, CSWL, CSAVL, and CSAVL-S

\begin{tabular}{llcccccc}
\hline word list & $\begin{array}{l}\text { test } \\
\text { format }\end{array}$ & types & lemmas & families & $\begin{array}{r}\text { CSAC2 } \\
\text { coverage }\end{array}$ & $\begin{array}{r}\text { coverage } \\
\text { per 100 } \\
\text { types }\end{array}$ & $\begin{array}{r}\text { coverage } \\
\text { per 100 } \\
\text { lemmas }\end{array}$ \\
\hline $\begin{array}{l}\text { CSWL } \\
\text { (Minshall, } \\
\text { 2013) }\end{array}$ & type & 2,033 & 1,225 & 432 & $5.57 \%$ & $0.27 \%$ & $0.45 \%$ \\
$\begin{array}{l}\text { CSAVL-S } \\
\text { POS }\end{array}$ & 1,398 & 702 & & $3.84 \%$ & $0.27 \%$ & $0.55 \%$ \\
\hline $\begin{array}{l}\text { AWL \& } \\
\text { CSWL }\end{array}$ & type & 4,969 & 3,151 & 1,002 & $17.26 \%$ & $0.35 \%$ & $0.55 \%$ \\
$\begin{array}{l}\text { CSAVL \& } \\
\text { CSAVL-S }\end{array}$ & POS & 3,119 & 1,606 & & & & \\
\end{tabular}

\subsection{General word list coverage comparisons for minimum reading comprehension}


As one of the research purposes of this project (stated in Section 2.10), a primary goal for the development of the CSAVL was to combine the list with a general highfrequency list in an attempt to reach the 95\% coverage threshold suggested by Laufer (1989, 2010) for a minimal level of reading comprehension. This section reports the results of coverage tests that were performed using various combinations of general highfrequency word lists, the CSAVL, and the two supplemental word lists that were compared in the previous section (the CSAVL-S and CSWL). These tests were performed in order to determine whether the CSAVL and CSAVL-S, when combined with a general high-frequency word list, could reach the 95\% coverage threshold necessary to provide a minimal level of reading comprehension of academic CS texts for English language learners. My analysis demonstrates that the CSAVL/CSAVL-S, when combined with a general high-frequency list, is able to reach the 95\% threshold. My analysis also demonstrates that the new-GSL/CSAVL/CSAVL-S combination is able to approach the $95 \%$ threshold using less than half the number of lemmas contained in the GSL/AWL/CSWL.

The first two rows of Table 4.3 list the CSAC2 coverage of two general highfrequency word lists, the General Service List (West, 1953) (GSL) and the New General Service List (Brezina \& Gablasova, 2015) (new-GSL). The new-GSL was found to have higher coverage of the CSAC2 corpus (83.32\%) than the GSL (78.61\%) while containing less than half the number of lemmas.

The third and fourth rows of Table 4.3 list the coverage results of combination of the GSL/AWL and the combination of the new-GSL/CSAVL. When the new-GSL and 
CSAVL were combined and duplicate lemmas were removed, they provided a coverage of $90.88 \%$ of the corpus, very similar to the $90.81 \%$ coverage provided by the GSL/AWL combination. The 3,201-lemma new-GSL/CSAVL combination was the more efficient pairing, providing similar total coverage with less than half the number of lemmas of the 7,264-lemma GSL/AWL combination.

The fifth and sixth rows of Table 4.3 list the results of coverage tests performed with the three-list combinations: the GSL/AWL/CSWL and the new-GSL/CSAVL/CSAVL-S. The three-list combination of the GSL/AWL/CSWL covered 95.49\% of the CSAC2, surpassing Laufer’s (1989, 2010) 95\% lexical threshold for minimal comprehension. This was slightly higher than the coverage results reported by Minshall (2013), who found this combination to cover $95.11 \%$ of his own CS corpus and $94.41 \%$ of his test CS corpus. The three-list combination of the new-GSL/CSAVL/CSAVL-S was found to cover $94.77 \%$ of the CSAC2, achieving coverage within $0.23 \%$ of the lexical threshold. The new-GSL/CSAVL/CSAVL-S combination, totaling 3,918 lemmas, was able to achieve similar coverage to the 8,489-lemma GSL/AWL/CSWL combination with less than half the number of lemmas. 
Table 4.3 CSAC2 coverage of general frequency list combinations

\begin{tabular}{llcccccc}
\hline word list & $\begin{array}{l}\text { test } \\
\text { format }\end{array}$ & types & lemmas & families & $\begin{array}{r}\text { CSAC2 } \\
\text { coverage }\end{array}$ & $\begin{array}{r}\text { coverage } \\
\text { per 100 } \\
\text { types }\end{array}$ & $\begin{array}{r}\text { coverage } \\
\text { per 100 } \\
\text { lemmas }\end{array}$ \\
\hline $\begin{array}{l}\text { GSL (West, } \\
\text { 1953) }\end{array}$ & type & 7,822 & 5,338 & 2,000 & $78.61 \%$ & $1.00 \%$ & $1.47 \%$ \\
$\begin{array}{l}\text { new-GSL } \\
\text { (Brezina \& }\end{array}$ & POS & 5,115 & 2,495 & & $83.32 \%$ & $1.63 \%$ & $3.34 \%$ \\
$\begin{array}{l}\text { Gablasova, } \\
\text { 2015) }\end{array}$ & & & & & & & \\
\hline $\begin{array}{l}\text { GSL \& AWL } \\
\text { type }\end{array}$ & 10,904 & 7,264 & 2,570 & $90.81 \%$ & $0.83 \%$ & $1.25 \%$ \\
$\begin{array}{l}\text { new-GSL \& } \\
\text { CSAVL }\end{array}$ & POS & 6,465 & 3,201 & & $90.88 \%$ & $1.41 \%$ & $2.84 \%$ \\
\hline $\begin{array}{l}\text { GSL, AWL \& } \\
\text { CSWL }\end{array}$ & type & 12,778 & 8,489 & 3,002 & $95.49 \%$ & $0.75 \%$ & $1.12 \%$ \\
$\begin{array}{l}\text { new-GSL, } \\
\text { CSAVL \& } \\
\text { CSAVL-S }\end{array}$ & POS & 7,663 & 3,918 & & $94.77 \%$ & $1.24 \%$ & $2.42 \%$ \\
\hline
\end{tabular}

In order to determine which general service list, when combined with the CSAVL/CSAVL-S, would result in the highest total coverage and greatest efficiency, the CSAVL/CSAVL-S was also combined with the Browne (2014) New General Service List (NGSL). As was described in Section 3.15, the NGSL was originally organized in the counting unit of the modified lemma. The type version of this list, which is available as an AntWordProfiler (Anthony, 2008) supplement, was converted to lemma form using Lancsbox (Brezina et al., 2015, 2018) before combining it with the CSAVL/CSAVL-S and removing all duplicate lemmas.

In order to also test the accuracy of the type-to-lemma conversion procedure that was used in this study, NGSL coverage of CSAC2 was tested using both type-based and POS-based methods. As shown in the first two rows of Table 4.4, The type version of the 
NGSL covered $88.34 \%$ of the CSAC2, while the lemma version covered $87.4 \%$. This difference of $0.94 \%$ between the POS and type test results was likely due to inconsistencies in the Lancsbox part-of-speech tagging system, Treetagger (Schmid, 1994, 1995), which was found to identify items with_ing suffixes as nouns, verbs, or both, with some inconsistency. However, despite the fact that the type lists that were being provided to the tagger were lists of words that were out of sentential context, the tagger still performed well enough to yield POS coverage results that were within $1 \%$ of the type coverage tests. The difference in coverage between these two methods demonstrates that a more accurate tool for converting wordlists into lemma form may be useful to future word list development and comparison.

As the second row of Table 4.4 shows, the NGSL covered $87.4 \%$ of the CSAC2 test corpus. The 4,770-lemma NSGL's coverage of $87.4 \%$ exceeded the $83.32 \%$ achieved by the 2,495-lemma new-GSL (shown in Table 4.3). However, when comparing the two lists by lemma count, the 2,495-lemma new-GSL was the noticeably more efficient word list, providing comparable coverage of a CS corpus while being only 52\% of the size of the 4,770-lemma NGSL.

The fourth row of Table 4.4 shows the results of the coverage test performed with Browne's NGSL combined with the CSAVL and CSAVL-S (with duplicate lemmas removed). This combination covered $94.96 \%$ of the CSAC2, coming within $0.04 \%$ of the lexical threshold. When designing the NGSL, Browne (2014) omitted numbers (“two”), days of the week ("Monday”), and months ("May”) from the main list and instead placed these items on a 174-type NGSL supplemental list. When this supplemental list was 
combined with the NGSL/CSAVL/CSAVL-S, it provided 95.46\% coverage of the CSAC2 (shown on the fifth row of Table 4.4). This demonstrated that the CSAVL/CSAVL-S can be combined with a general list to reach the lexical threshold suggested by Laufer (1989, 2010) for a minimal level of reading comprehension.

Table 4.4 CSAC2 coverage of NGSL and CSAVL combinations

\begin{tabular}{llllrrr}
\hline word list & $\begin{array}{l}\text { test } \\
\text { format }\end{array}$ & types & lemmas & $\begin{array}{r}\text { CSAC2 } \\
\text { coverage }\end{array}$ & $\begin{array}{r}\text { coverage per } \\
100 \text { types }\end{array}$ & $\begin{array}{r}\text { coverage } \\
\text { per 100 } \\
\text { lemmas }\end{array}$
\end{tabular}

\begin{tabular}{lcccccc}
\hline $\begin{array}{l}\text { NGSL (Browne, } \\
\text { 2014) }\end{array}$ & type & 8,480 & 4,770 & $88.34 \%$ & $1.04 \%$ & $1.85 \%$ \\
\hline NGSL & POS & 8,480 & 4,770 & $87.40 \%$ & $1.03 \%$ & $1.83 \%$ \\
\hline NGSL \& CSAVL & POS & 9,318 & 5,195 & $91.13 \%$ & $0.98 \%$ & $1.75 \%$ \\
$\begin{array}{l}\text { NGSL, CSAVL \& } \\
\begin{array}{l}\text { CSAVL-S } \\
\text { NGSL, CSAVL, }\end{array}\end{array}$ & POS & 10,332 & 5,902 & $94.96 \%$ & $0.92 \%$ & $1.61 \%$ \\
$\begin{array}{l}\text { CSAVL-S \& NGSL } \\
\text { supplement }\end{array}$ & & 10,502 & 6,060 & $95.46 \%$ & $0.91 \%$ & $1.58 \%$ \\
\hline
\end{tabular}

Although the four-list combination of the NGSL/CSAVL/CSAVL-S/NGSL supplement was able to reach the 95\% coverage threshold of a CS corpus, when it is compared with the new-GSL/CSAVL/CSAVL-S combination (as shown in rows two and three of Table 4.5), the NGSL combination is notably less efficient than the new-GSL one. Although the 6,060-lemma NGSL combination offers a 0.69\% total coverage increase and surpasses Laufer's lexical threshold, the 3,918-lemma new-GSL combination is able to provide comparable coverage of a CS corpus while containing only $65 \%$ of the total number of lemmas. Furthermore, research has not examined 
whether coverage differences of less than a percent have any impact on minimum reading comprehension. Thus, sacrificing $0.23 \%$ in coverage for a significant reduction in total lemmas is a reasonable trade-off.

Table 4.5 CSAC2 coverage of general, academic, and technical list combinations

\begin{tabular}{|c|c|c|c|c|c|c|}
\hline word list & $\begin{array}{l}\text { test } \\
\text { format }\end{array}$ & types & lemmas & $\begin{array}{r}\text { CSAC2 } \\
\text { coverage }\end{array}$ & $\begin{array}{r}\text { coverage per } \\
100 \text { types }\end{array}$ & $\begin{array}{r}\text { coverage } \\
\text { per } 100 \\
\text { lemmas }\end{array}$ \\
\hline GSL, AWL \& CSWL & type & 12778 & 8,480 & $95.49 \%$ & $0.75 \%$ & $1.12 \%$ \\
\hline $\begin{array}{l}\text { new-GSL, CSAVL \& } \\
\text { CSAVL-S }\end{array}$ & POS & 7663 & 3918 & $94.77 \%$ & $1.24 \%$ & $2.42 \%$ \\
\hline $\begin{array}{l}\text { NGSL, CSAVL, } \\
\text { CSAVL-S \& NGSL } \\
\text { supplement }\end{array}$ & POS & 10502 & 6060 & $95.46 \%$ & $0.91 \%$ & $1.58 \%$ \\
\hline
\end{tabular}

\subsection{Coverage across general, academic, and CS corpora}

After performing coverage tests using a variety of word lists on a single academic computer science corpus (the CSAC2), the CSAVL and CSAVL-S word lists were then tested against a variety of corpora types: a corpus of general English, a general academic corpus, and a second academic computer science corpus. The results of these tests demonstrated that: 1) The CSAVL and CSAVL-S are more representative of academic vocabulary than the vocabulary of general English; and 2) The CSAVL and CSAVL-S are representative of academic CS English usage beyond that found in the corpus from which they were extracted. 
When evaluating the Academic Vocabulary List (AVL), Gardner and Davies (2014) tested the word list on both a general and an academic corpus in order to demonstrate that the AVL contained items that more frequently appeared in academic English than in general usage. Lei and Liu (2016) followed Gardner and Davies’ example, using the British National Corpus (BNC) academic and non-academic sections along with academic medical corpora to provide evidence that their Medical Academic Vocabulary List (MAVL) was an academic word list, and more specifically, a medical academic word list. The same test was used for evaluating the CSAVL and CSAVL-S, using the BNC academic and non-academic sections.

The third column of Table 4.6 shows that the CSAVL covered only $2.96 \%$ of the BNC non-academic portion lemmas, somewhat similar to the $3.69 \%$ reported by Lei and Liu for the MAVL. For the academic portion of the BNC, the CSAVL covered $4.93 \%$, which suggests that the items in the CSAVL appear more often in an academic context than a general one. The CSAVL-S covered $0.62 \%$ of the BNC non-academic, which was comparable to the $0.39 \%$ reported by Minshall when testing the CSWL technical word list on a fiction corpus. This increased to $0.9 \%$ when comparing the CSAVL-S to the academic portion, also suggesting that the list contents are more representative of academic than general vocabulary. 
Table 4.6 Coverage of the CSAVL and CSAVL-S in the BNC academic and nonacademic portions

\begin{tabular}{llrr}
\hline word list & test format & BNC non-academic & BNC academic \\
& & & \\
\hline CSAVL & POS & $2.96 \%$ & $4.93 \%$ \\
CSAVL-S & POS & $0.62 \%$ & $0.90 \%$ \\
new-GSL & POS & $80 \%$ & $83.44 \%$ \\
CSAVL \& CSAVL-S & POS & $3.58 \%$ & $5.83 \%$ \\
new-GSL, CSAVL \& CSAVL-S & POS & $81.74 \%$ & $86.57 \%$ \\
\hline
\end{tabular}

For the task of identifying technical vocabulary, Nation and Chung (2004) suggested four techniques: employing discipline experts to rate word technicality, using contextual clues within the text, use of a technical dictionary, and computer-based corpus approaches. In creating the Computer Science Word List (CSWL), Minshall (2013) adopted the latter two methods when evaluating word technicality. A corpus-based method used by Minshall was the coverage comparison of the word list in the primary corpus with those observed in a secondary test CS corpus. To similarly evaluate the CSAVL, the CSAVL's coverage of the CSAC1 (the corpus from which the list was extracted) and CSAC2 (test corpus) were compared.

Before the coverage tests were performed, the CSAC1 corpus was processed in the same manner used for the CSAC2 (as described in Section 4.3). It was found (as shown in Table 4.7) that the CSAVL covered a similar portion of both the CSAC1 (16.87\%) and CSAC2 (16.06\%), and the CSAVL-S covered $4.17 \%$ of the CSAC1 and $3.84 \%$ of the CSAC2. When combined with the new-GSL, the total coverage of the three lists in the CSAC1 was only .08\% higher than their coverage of the CSAC2. The similarity of these 
results suggests that the CSAVL/CSAVL-S lists are representative of academic CS English usage beyond that found in the corpus from which they were extracted.

Additionally, the higher coverage of the CSAVL/CSAVL-S in CS corpora compared with their coverage of academic and general corpora provides evidence that these lists do include CS-specific technical vocabulary. The technicality of these items is further explored using the technical dictionary method (as suggested by Nation and Chung) in the next section.

Table 4.7 CSAVL coverage of computer science corpora

\begin{tabular}{llrr}
\hline word list & $\begin{array}{l}\text { test } \\
\text { format }\end{array}$ & CSAC1 & CSAC2 \\
& POS & $16.87 \%$ & $16.06 \%$ \\
\hline CSAVL & POS & $4.17 \%$ & $3.84 \%$ \\
CSAVL-S & POS & $82.69 \%$ & $83.32 \%$ \\
new-GSL & POS & $21.04 \%$ & $19.90 \%$ \\
CSAVL \& CSAVL-S & $94.79 \%$ & $94.71 \%$ \\
\hline new-GSL, CSAVL \& CSAVL-S & POS & & \\
\hline
\end{tabular}

\subsection{Content comparison with technical dictionaries}

The CSAVL and CSAVL-S were designed to be used as specialist word lists for the specific academic discipline of computer science. In order to determine whether or not the CSAVL and CSAVL-S can be considered specialist lists containing technical vocabulary, Nation and Chung’s (2004) method of identifying technical vocabulary through comparison with a technical dictionary was employed. This section presents the results of CSAVL and CSAVL-S content comparisons with the entries of technical 
dictionaries in order to evaluate the degree of the lists' technicality. The results demonstrate that the CSAVL and CSAVL-S contain a larger proportion of technical CS items than the Computer Science Word List (Minshall, 2013), a specialist list which was specifically designed to represent CS technical vocabulary.

For the comparison of the CSAVL and CSAVL-S with a technical dictionary, the Oxford Concise Dictionary of Mathematics (ODOM) (2009) was used in addition to the Oxford Dictionary of Computer Science (ODOCS). Numerous mathematical terms such as “multiplication_n”, “finite_adj”, and “coefficient_n” were found in early examinations of the contents of the CSAVL and CSAVL-S. The appearance of these items was not surprising since three of the sub-corpora that make up CSAC1 are explicitly mathematical: Mathematics of Computing (ACM articles), Mathematics of Computing (textbooks), and Probability and Statistics (textbooks). Mathematical content was also found throughout the Algorithms (textbooks), Theory of Computation (ACM articles), and Theory of Computation (textbooks) sub-corpora. For these reasons, a mathematics dictionary was also chosen for this investigation into the technicality of the word list contents. Since the lists were intended for the primary purpose of CS context-specific usage, only the ODOCS was used as part of the word list filtering criteria (described in Section 3.9). However, by also using the ODOM to identify and label mathematical terms on the lists, I hoped to provide list-users with additional contextual clues regarding how these terms were used within the mathematical sub-corpora of the CSAC1.

I developed a program in order to identify the entries of the two dictionaries which matched lemmas on the word lists. Only exact matches were considered, and since the 
word lists contained only single-word items, all multi-word entries in the dictionaries were ignored (the CSAVL lemma "access_v" was considered to be a match with ODOCS entry “access”, but not a match with “access time”). Minshall’s (2014) Computer Science Word List (CSWL) was also examined for ODOCS/ODOM overlap in order to make comparisons with an existing CS wordlist that had already been determined by Minshall to be a technical CS word list (following Chung and Nation’s criteria).

Figures 4.1 and 4.2 show the results of the comparisons between three word lists: the CSAVL, CSAVL-S, and CSWL (Minshall, 2013), and the ODOCS and ODOM technical dictionaries. The three circular pie charts represent the total lemma counts of the CSAVL, CSAVL-S, and CSWL word lists and are divided into lemmas that did/did not appear as entries in the technical dictionaries. The bars to the right of each pie chart represent which technical dictionary the matching lemmas were found in. The left pie chart of Figure 4.1 depicts the 904 lemmas of the CSAVL. Of the 904 total lemmas on the CSAVL word list, 48\% did not appear in the technical dictionaries, 9\% appeared only in the ODOM, 32\% appeared only in the ODOCS, and 11\% appeared in both dictionaries. The right pie chart of Figure 4.1 depicts the 702 lemmas of the CSAVL-S supplemental list. Of the 702 total lemmas on the CSAVL-S, 59\% did not appear in the technical dictionaries, 9\% appeared only in the ODOM, 25\% appeared only in the ODOCS, and 7\% appeared in both dictionaries. The CSAVL-S supplement, which included items that were less frequent and less evenly distributed in the CSAC1 corpus than those found in the main CSAVL list, contained 11\% fewer technical dictionary items than the CSAVL. The finding that the CSAVL list contains more technical dictionary entries than the 
CSAVL-S supplement is not entirely surprising given that the CSAVL is a list of the most frequently used academic CS-specific items by design, and this was also a likely motivation for the selection of the entries which were included in the ODOCS.

\section{CSAVL}

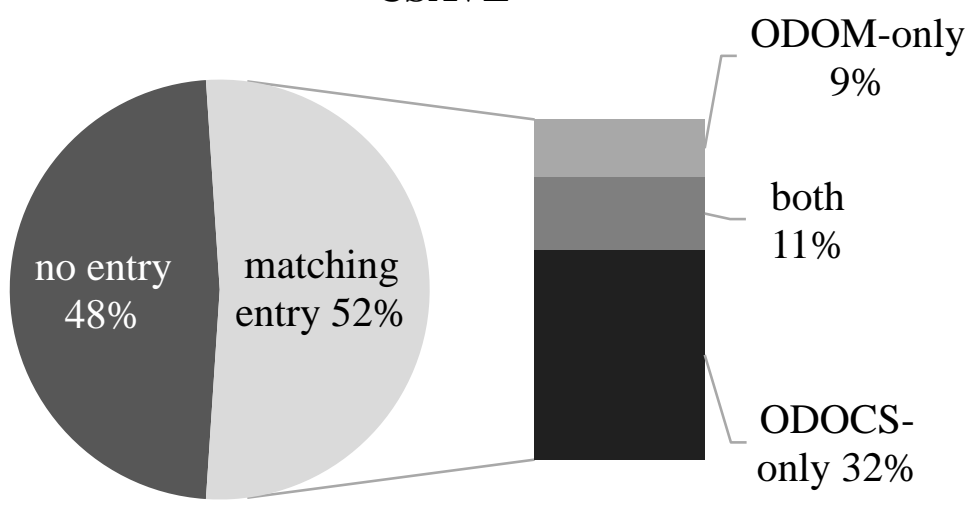

\section{CSAVL-S}

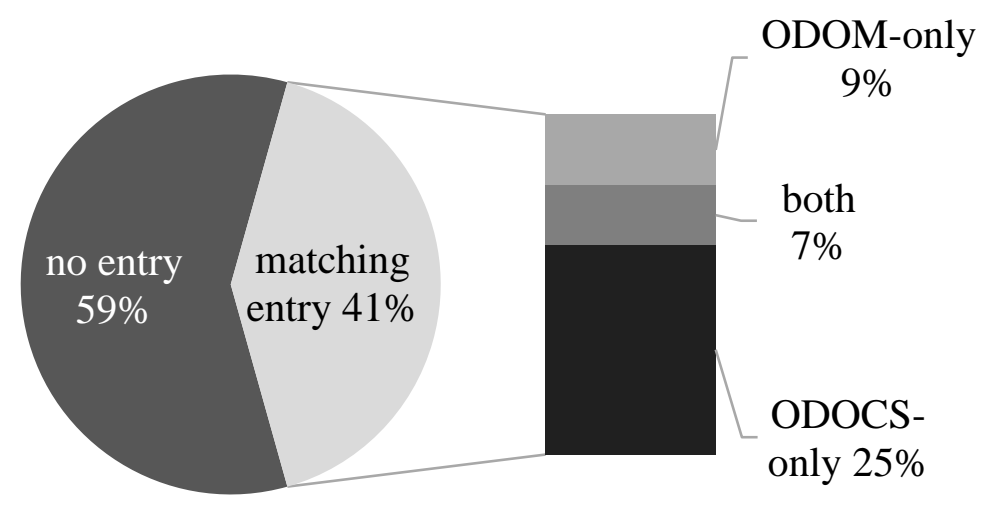

Figure 4.1 Percentages of CSAVL word list lemmas matching technical dictionary entries

Note: Each circular pie chart represents the total lemma count of its respective word list. The bar to the right of each pie chart represents the portion of word list lemmas that matched technical dictionary entries. 
When Minshall (2014) evaluated the technicality of the Computer Science Word List (CSWL) by comparing it with the ODOCS, he found that 229 of the CSWL word family headwords (52.9\%) were ODOCS entries. In order to provide a closer comparison with the CSAVL, the CSWL was converted to lemma form before identifying matching entries in the ODOCS and ODOM. Comparisons of the lists in lemma form to the dictionary entries may be considered a more appropriate form of comparison since the ODOCS and ODOM contain separate entries for items that would be grouped together under the word family system (“address”, “addressing”, “close”, “closed”). As shown in Figure 4.2, it was found that in lemma form, only 26\% of the CSWL lemmas appeared as technical dictionary entries in the ODOCS or ODOM. The results of this comparison suggest that both the CSAVL and CSAVL-S contain a larger proportion of technical CS items than the CSWL, a specialist list which was specifically designed to represent CS technical vocabulary. 


\section{CSWL}

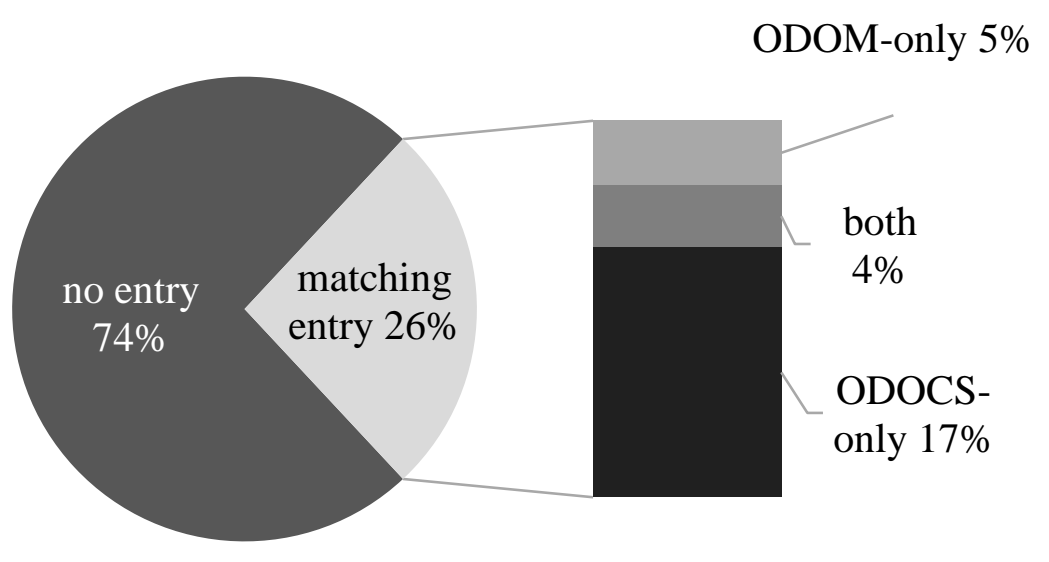

Figure 4.2 Percentages of CSWL word list lemmas matching technical dictionary entries

Note: Each circular pie chart represents the total lemma count of its respective word list. The bar to the right of each pie chart represents the portion of word list lemmas that matched technical dictionary entries.

\subsection{Word list content comparisons}

One of the stated research purposes for this project (listed in Section 2.10) was to compare the contents of the CSAVL and CSAVL-S with that of other similarly-purposed word lists. In this section (4.6), the contents of six word lists (the GSL/AWL/CSWL and the new-GSL/CSAVL/CSAVL-S) are compared for the following purposes: 1) to point out the similarities and differences between the CSAVL/CSAVL-S and previous lists with a similar purpose; 2) to provide a more thorough description of the CSAVL and CSAVL-S; and 3) to gain a better understanding of how lemmas were distributed across 
the general, academic, and supplemental divisions of the new-GSL/CSAVL/CSAVL-S combination.

To examine the similarities and differences between the contents of these word lists, an analysis of overlapping lemmas between lists was conducted. The overlapping items were identified by creating a simple program that output lists of overlapping and nonoverlapping items between word lists. The results of this analysis are depicted in Figures 4.3, 4.4, and 4.5.

Figure 4.3 depicts the 2,495 lemmas of the Brezina and Gablasova’s (2015) newGSL. The new-GSL was the general service list that was used to partially filter highfrequency content from the CSAVL (as described in Section 3.9). Because of this list's close relationship to the CSAVL, it was also included in the analysis of overlapping content that is presented in this section. Figure 4.2 shows that of the 2,495 new-GSL lemmas, 75\% were found to overlap with GSL lemmas, 18\% with AWL lemmas, and 2\% with CSWL lemmas. These results were similar to those reported by Brezina and Gablasova (2015) in their own evaluation of the new-GSL's contents. The GSL overlap is not surprising for a general service list such as the new-GSL. However, the overlap with AWL content reflects the design differences of the new-GSL from West's GSL as well as the changes in the usage of English that have occurred in the time since the creation of the GSL. In their evaluation of the new-GSL, Brezina and Gablasova argued that the AWL content that appeared in the new-GSL (including arguably commonplace items such as “team_n”, “computer_n”, and “job_n”) should have been excluded from an academic word list such as the AWL, and instead placed on a general high-frequency 
word list. Items such as “computer_n” are noticeably absent from West’s (1953) GSL. The absence of high-frequency lemmas representative of contemporary English, such as “Internet_n” and "kid_n”, points toward the need for new general service lists such as the new-GSL or NGSL (Browne, 2014) that might better represent the language of contemporary general high-frequency English. Because of the new-GSL’s representativeness of contemporary high-frequency English, its lemma-based structure, and its ability to provide efficient coverage of a variety English corpora (Brezina \& Gablasova, 2015; Browne, 2014), the new-GSL was selected as the general highfrequency list that was used to create the CSAVL.

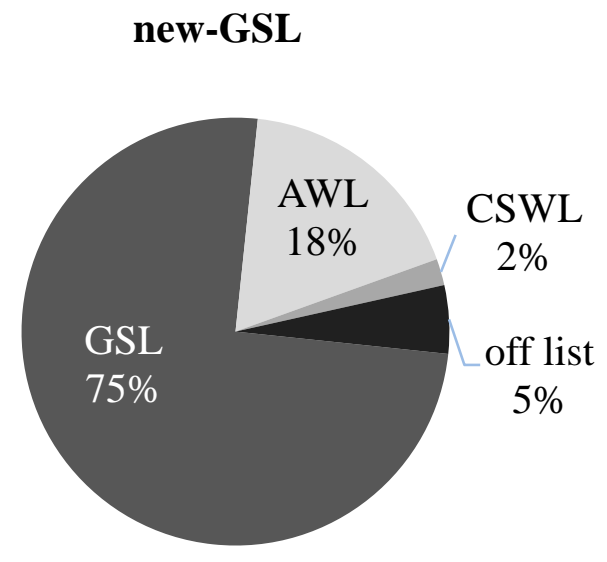

Figure 4.3 new-GSL lemma overlap with the GSL, AWL, and CSWL

Figure 4.4 depicts the 904 lemmas of the CSAVL. Of the 904 lemmas of the CSAVL, 37\% overlapped with GSL lemmas, 37\% with AWL lemmas, and 23\% with CSWL lemmas. The CSAVL overlap with the Academic Word List (AWL) and Computer Science Word List (CSWL) is not surprising for a list that was intended to 
represent the vocabulary of academic computer science. The presence of GSL highfrequency items on the CSAVL can be explained by the CSAVL word selection criteria that allowed for the inclusion of high-frequency items that either: 1) matched entries in a CS technical dictionary; or 2) were used more frequently in computer science contexts than general usage contexts (these word selection criteria are listed in Section 3.9). Of the GSL lemmas that overlapped with the contents of the CSAVL, 199 were lemmas with CS-specific meanings and matching ODOCS entries (such as "server_n" and "tree_n”), and the remaining 135 appeared in the CS corpus that was used to create the CSAVL at a higher relative frequency than in a general English corpus (items such as “efficiency_adj” and "false_adj”). CSAVL items such as "server_n”, which might be considered highly relevant to English learners focusing on CS, were omitted by Minshall (2013) from the CSWL due to the presence of the word family "serve" on the GSL, a design decision which limited the CSWL in its description of the vocabulary of academic CS. By allowing GSL items such as "server_n" and "tree_n" into the CSAVL and explicitly marking these items as having CS-specific meanings, the CSAVL describes CS vocabulary in a more detailed and context-specific way than the AWL or CSWL. The 3\% of the CSAVL lemmas that did not overlap with any GSL/AWL/CSWL contents included technical ODOCS items, such as "website_n" and "pop_v" (to "pop” a stack of data is to remove the top item), as well as mathematical ODOM items such as "subtract_v". 


\section{CSAVL}

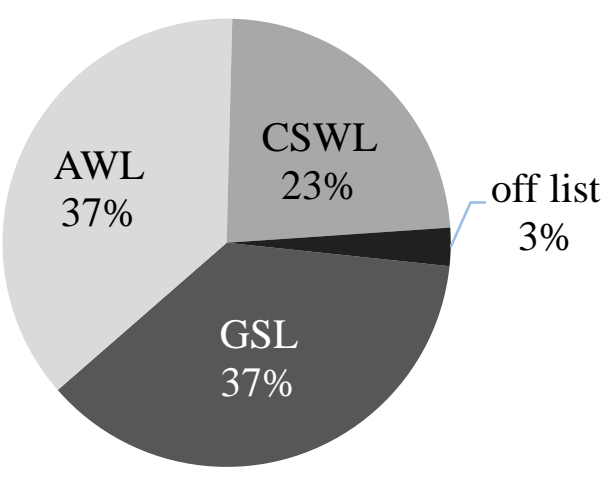

Figure 4.4 CSAVL lemma overlap with the GSL, AWL, and CSWL

Figure 4.5 depicts the 702 lemmas of the CSAVL-S supplemental list. Of the 702 lemmas that make up the CSAVL-S, 29\% overlapped with GSL lemmas, 19\% with AWL lemmas, and 29\% with CSWL lemmas. Of the GSL lemmas in the CSAVL-S, 74 were ODOCS entries with CS-specific meanings (such as “string_n”, “leaf_n”, and “worm_n”), and the other 128 appeared in the CS corpus at a higher relative frequency than in a general English corpus, including items such as “thread_n“, “wrapper_n”, and “crack_v”. Although GSL lemmas such as "thread_n” did not have single-word matching ODOCS entries, they partially matched other ODOCS entries such as “autothread”, “multithreading”, “single threading”, “threading”, and "threaded list”, which points toward the likelihood that items such as "thread_n” have CS-specific meanings despite their exclusion from the ODOCS as a single-word entry.

The 23\% of the CSAVL-S that did not overlap with the contents of the GSL, AWL, or CSWL (the 161 “off list” lemmas) included items with ODOCS entries such as 
“blog_n”, “malware_n“, and “phishing_n” as well as arguably CS-related items omitted by the ODOCS such as "menu_n” and “tweet_v”. The "off list” portion of the CSAVL-S, a list which was designed to include items that were clustered in specific CS subdisciplines, also included terms that are likely to be more specific to CS sub-disciplines such as security (“privilege_n”), hardware (“transistor_n”), and wireless networking (“jamming_n”). These items may have been omitted from Minshall’s CSWL list because of his use of a range criteria (items had to appear in at least five out of his ten subdisciplinary categories) that was designed to limit the inclusion of CS terms that were more specific to certain CS sub-disciplines.

\section{CSAVL-S}

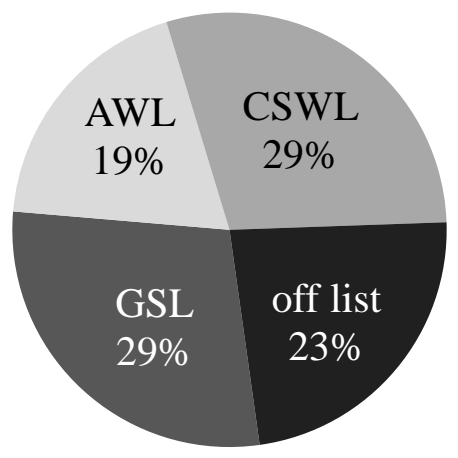

Figure 4.5 CSAVL-S lemma overlap with the GSL, AWL, and CSWL 


\subsection{Coverage tests of overlapping content}

In the previous section, the overlapping content between six word lists, the GSL/AWL/CSWL and the new-GSL/CSAVL/CSAVL-S, was identified. The distribution of overlapping contents (depicted in Figures 4.2, 4.3, and 4.4) showed that the majority of the lemmas in the new-GSL/CSAVL/CSAVL-S combination could also be found in the GSL/AWL/CSWL combination. The high degree of overlap, along with the efficiency of the new-GSL/CSAVL/CSAVL-S in covering a CS corpus (demonstrated in Section 4.4), suggest that the majority of the content of the new-GSL/CSAVL/CSAVL-S is not "new" content, but is instead the subset of GSL/AWL/CSWL lemmas that most efficiently describe the language of written academic computer science.

Although the GSL/AWL/CSWL combination was able to cover more than 95\% of a corpus of CS texts, it did so far less efficiently than the new-GSL/CSAVL/CSAVL-S combination. The GSL/AWL/CSWL's relative inefficiency in covering a CS corpus may lead word list users to wonder how relevant much of the content of the GSL/AWL/CSWL is for learners who have the specific goal of developing CS comprehension. To answer this question, an additional series of coverage tests was performed. This series of coverage tests was performed on the CSAC2 corpus using two portions of the GSL, AWL, and CSWL word lists: the portions that overlapped with newGSL/CSAVL/CSAVL-S lemmas, and the portions that did not. By comparing the coverage of a CS corpus provided by the two portions of each word list, we can gain an 
indication of what degree the lemmas of each portion might be relevant to English learners who are reading academic computer science texts.

Table 4.9 lists the results of coverage tests performed on the CSAC2 corpus using the portions of the GSL, AWL, and CSWL that overlapped with the lemmas of the newGSL/CSAVL/CSAVL-S and the portions that did not. The first and second rows of Table 4.9 list the coverage of the CS corpus provided by two portions of the GSL. Of the 5,338 total lemmas on the GSL, 2,299 (43\%) overlapped with the new-GSL/CSAVL/CSAVLS. This overlapping portion of the GSL was found to cover $76.93 \%$ of the CS corpus. The 3,040 lemmas (57\%) of the GSL that did not overlap with the newGSL/CSAVL/CSAVL-S covered only $0.96 \%$ of the CS corpus. The less than $1 \%$ coverage of a CS corpus provided by this large portion of the GSL suggests that more than half of the lemmas contained in the GSL may have little or no representation in the vocabulary of academic CS texts.

The third and fourth rows of Table 4.9 list the coverage of a CS corpus provided by two portions of the AWL. Of the 1,926 total lemmas on the AWL, 832 (43\%) overlapped with the new-GSL/CSAVL/CSAVL-S. The overlapping portion of the AWL covered $11.67 \%$ of the corpus, whereas the 1,094 lemmas (57\%) of the AVL that did not overlap covered $0.53 \%$ of the CS corpus. The off-list portion of the AWL was only able to cover approximately half of one percent of the CS corpus, which demonstrates that the majority of the lemmas of the AWL had little or no representation in the academic CS texts that made up the CSAC2. 
The fifth and sixth rows of Table 4.9 list the coverage of a CS corpus provided by two portions of the CSWL. Of the 1,225 lemmas of the CSWL, 455 lemmas (37\%) overlapped with the new-GSL/CSAVL/CSAVL-S. The overlapping portion of the CSWL was able to provide $5.18 \%$ coverage of the CS corpus. The 722 CSWL lemmas (63\%) that did not overlap provided only $0.36 \%$ coverage of the CS corpus. Although the CSWL was designed to be a list of computer science technical vocabulary, the majority of its lemmas were found to have little or no representation in the Computer Science Academic Corpus 2 (CSAC2).

Table 4.8 CSAC2 coverage of overlapping and off-list portions of the GSL, AWL, and CSWL

\begin{tabular}{llcc}
\hline word list & $\begin{array}{l}\text { test } \\
\text { format }\end{array}$ & $\begin{array}{r}\text { number of } \\
\text { lemmas }\end{array}$ & $\begin{array}{r}\text { CSAC2 } \\
\text { coverage }\end{array}$ \\
\hline $\begin{array}{l}\text { GSL lemmas overlapping with new- } \\
\text { GSL / CSAVL / CSAVL-S }\end{array}$ & POS & $2,299(43 \%)$ & $76.93 \%$ \\
GSL off-list lemmas & POS & $3,040(57 \%)$ & $0.96 \%$ \\
$\begin{array}{l}\text { AWL lemmas overlapping with new- } \\
\text { GSL / CSAVL / CSAVL-S }\end{array}$ & POS & $832(43 \%)$ & $11.67 \%$ \\
AWL off-list lemmas & POS & $1,094(57 \%)$ & $0.53 \%$ \\
$\begin{array}{l}\text { CSWL lemmas overlapping with new- } \\
\text { GSL / CSAVL / CSAVL-S }\end{array}$ & POS & $455(37 \%)$ & $5.18 \%$ \\
\hline CSWL off-list lemmas & POS & $772(63 \%)$ & $0.36 \%$ \\
\hline
\end{tabular}

The surprisingly low coverage of a CS corpus that was provided by the CSWL offlist lemmas can be partially explained by the word family-to-lemma conversion process. While the CSWL in word family form only includes headwords such as "execute”, after 
converting to lemma form, lemmas that are less relevant to CS, such as “executioner_n”, are generated. While this may help to explain the low coverage of a CS corpus provided by the majority of the lemmas on the CSWL, the lack of specificity provided by a word family headword such as "execute” still remains as a challenge for word list users to deal with. The CSWL provides no indication of the presence of CS-specific meanings or any indication of which parts of speech or derivational forms are most frequent in CS contexts, leaving list users with the responsibility of performing their own research or making guesses as to which meanings and forms are relevant to the context of academic CS.

The stark differences in the amount of coverage of a CS corpus provided by the two portions of the GSL, AWL, and CSWL that were compared in this section demonstrate that the new-GSL, CSAVL, and CSAVL-S word lists contain the GSL/AWL/CSWL lemmas that are most relevant to academic computer science texts. The results in this section also further demonstrated the inefficiency of the GSL, AWL, and CSWL in covering an academic CS corpus, with more than half of the lemmas of each list providing only minimal amounts of coverage.

\subsection{Summary of results}

In the results chapter, I evaluated and described the CSAVL and CSAVL-S through a series of coverage and content comparisons with other word lists having similar 
purposes. The CSAVL and CSAVL-S word lists: 1) are able to provide efficient coverage of academic computer science texts; 2) when combined with a general service list, are able to approach the 95\% coverage threshold suggested by Laufer $(1989,2010)$ for a minimal level of reading comprehension; 3) can be considered to be both academic word lists and specialist lists of technical vocabulary; and 4) are primarily composed of the GSL/AWL/CSWL lemmas that are most representative of the vocabulary of academic computer science.

The ability of the CSAVL and CSAVL-S to provide efficient coverage of academic CS texts was demonstrated in the first two sections of the chapter (4.1 and 4.2). By comparing the results of coverage tests on a CS corpus using the CSAVL and two academic word lists, the AVL (Gardner \& Davies, 2014) and the AWL (Coxhead, 2000), it was demonstrated that the CSAVL was able to provide nearly three times as much normed coverage as the AVL and AWL word lists. When the CSAVL-S supplemental list was compared another CS-specific supplemental list, the CSWL (Minshall, 2013), it was found that the CSAVL-S provided similar normed coverage of a CS corpus to the CSWL. When the CSAVLICSAVL-S combination was compared to the AWL\CSWL combination, it was demonstrated that the CSAVL\CSAVL-S combination provided more than double the normed coverage of the AWLICSWL combination in a corpus of academic CS texts.

The ability of the CSAVL and CSAVL-S, in combination with a general service list, to reach the 95\% coverage threshold suggested by Laufer $(1989,2010)$ for a minimal level of reading comprehension was demonstrated in Section 4.3. In the coverage tests 
performed on the corpus of academic CS texts, it was found that the combination of the new-GSL/CSAVL/CSAVL-S was able to come within $0.23 \%$ of the $95 \%$ lexical threshold. It was also shown that the new-GSL/CSAVL/CSAV-S combination was able to approach the lexical threshold using less than half the number of lemmas contained in the GSL/AWL/CSWL, demonstrating the efficiency of this combination in covering a corpus of academic CS texts.

In Sections 4.4 and 4.5, I demonstrated that the CSAVL and CSAVL-S can be considered both academic vocabulary lists and lists of technical vocabulary. By performing coverage tests on two types of corpora, a general academic corpus and a general English corpus, using the CSAVL and CSAVL-S, it was demonstrated that the lists were more representative of academic vocabulary than the vocabulary of general English. By performing coverage tests with a second CS corpus and also comparing the contents of the CSAVL and CSAVL-S with technical dictionary entries, it was demonstrated that both the CSAVL and CSAVL-S could be considered specialist lists of technical vocabulary.

In Sections 4.6 and 4.7, it was also shown that the CSAVL and CSAVL-S are composed of the GSL/AWL/CSWL lemmas that are highly representative of the vocabulary of academic computer science. By comparing the content of the new-GSL, CSAVL, and CSAVL-S with the content of the GSL, AWL, and CSWL, and identifying overlapping lemmas, I demonstrated that the new-GSL, CSAVL, and CSAVL-S are primarily composed of lemmas that can be found in the GSL, AWL, and CSWL. By performing coverage tests using overlapping and non-overlapping portions of the lists, it 
was also demonstrated that the content of the new-GSL/CSAVL/CSAVL-S consists of the GSL/AWL/CSWL lemmas that can be most often found in a corpus of academic CS texts. 


\section{Chapter 5: Discussion}

The discussion chapter begins with a final evaluation of the Computer Science Academic Vocabulary List (CSAVL) and its supplemental list (the CSAVL-S) based on the results of the coverage and content comparisons that were reported in the previous chapter. This evaluation begins with Section 5.1, which contains a discussion of the ability of the CSAVL and CSAVL-S to provide efficient coverage of a corpus of academic computer science texts. This is followed by Section 5.2, which contains a review and assessment of the findings regarding the CSAVL and CSAVL-S' representativeness of the vocabulary of academic computer science and the technicality of the lists' contents. The evaluation of list representativeness and technicality is followed by Section 5.3, which provides a discussion of the contents of the CSAVL/CSAVL-S in relation to the contents of the General Service List (GSL) (West, 1953), the Academic Word List (AWL) (Coxhead, 2000), and the Computer Science Word List (Minshall, 2013). Section 5.4 contains a discussion of the pedagogical implications of CSAVL project and suggestions for the practical usages of the CSAVL/CSAVL-S by educators, pedagogical material developers, and independent learners. The limitations of this study are presented in Section 5.5, suggestions for future research are given in Section 5.6, and the discussion chapter concludes in Section 5.6 with final thoughts on the CSAVL project. 


\subsection{The coverage and efficiency of the CSAVL and CSAVL-S}

In the first two sections of the results chapter (Sections 4.1 and 4.2), it was demonstrated that the Computer Science Academic Vocabulary List (CSAVL) and its supplemental list (the CSAVL-S) are able to provide efficient coverage of academic computer science texts. Efficient coverage was defined as the ability of a word list to provide coverage of a corpus that is comparable to the coverage provided by other lists, but with fewer total list items. A word list that can provide more efficient coverage of a corpus provides a better cost/benefit relationship for list users than other similarlypurposed lists. A list that provides efficient coverage of the target body of texts will likely result in a shorter time investment for learners seeking improved reading comprehension of those types of texts. Because the lists that were compared in this study described a similar range of vocabulary, but were of different sizes, normed coverage (coverage per 100 lemmas and coverage per 100 types) was used as a metric to determine each list’s efficiency in covering a corpus of academic CS texts

As reported in Section 4.1, it was found that the CSAVL was able to provide more efficient coverage of academic CS texts than two general academic vocabulary lists: The Academic Vocabulary List (AVL) (Gardner \& Davies, 2014) and The Academic Word List (AWL) (Coxhead, 2000). The coverage comparison of the CSAVL and Gardner and Davies’ Academic Vocabulary List (AVL) showed that the CSAVL provided nearly three times higher normed coverage of a CS corpus. The AVL, which is several times larger than the CSAVL, was able to provide slightly higher total coverage, but the AVL's 
ability to provide coverage of a CS corpus is also limited by its standalone design. By contrast, the CSAVL was designed to be supplemented with the CSAVL-S, which allows it to exceed the total coverage of a CS corpus provided by the AVL while still remaining more efficient.

The coverage comparisons of the Academic Word List (AWL) (Coxhead, 2000) and CSAVL showed that the CSAVL was able to provide more total coverage of a CS corpus than the AWL, while containing only half the number of lemmas. The CSAVL provided nearly three times higher normed coverage of a CS corpus than Coxhead's AWL. Coxhead restricted all General Service List (GSL) (West, 1953) content (some of the most highly-frequent words in the English language) from the AWL, which was a likely factor in the AWL's limited ability to provide coverage of the CS corpus. The restriction of GSL items from the AWL resulted in the omission of items with CS-specific meanings such as “memory_n”, “tree_n”, and “server_n”, which were some of the most frequent items in both the Computer Science Academic Corpus 1 and 2 (CSAC1 and CSAC2), and could be considered highly relevant to English learners intending to develop their reading comprehension of academic CS texts.

In the comparison of supplemental academic CS word lists (described in Section 4.2), it was found that the CSAVL-S supplemental list was independently able to provide slightly higher normed coverage (per 100 lemmas) of a CS corpus than The Computer Science Word List (CSWL) (Minshall, 2013). When the CSAVL/CSAVL-S were combined and compared to the combined AWL/CSWL, the CSAVL/CSAVL-S was found to be the more efficient combination. In an academic CS corpus, the 
CSAVL/CSAVL-S was able to provide double the normed coverage as that provided by the AWL/CSWL combination.

In the results chapter, it was also demonstrated that the CSAVL and CSAVL-S word lists, when combined with a general service list, are able to reach the $95 \%$ coverage threshold suggested by Laufer (1989, 2010) for a minimal level of reading comprehension. According to Nation (2001), readers who recognize 95\% of the tokens in a text are likely to have a minimal level of comprehension when reading that text, and this figure has been used as a target coverage figure in numerous specialist word list studies (Konstantakis, 2007; Lei \& Liu, 2016; Minshall, 2013; Ward, 1999, 2009). The ability of the CSAVL and CSAVL-S, combined with a general high-frequency word list, to reach 95\% coverage of a corpus of academic CS texts indicates that these word lists may be viable tools for developing reading comprehension of academic CS texts.

In Section 4.3, the CSAVL and CSAVL-S were combined with two different general service lists: Brezina and Gablasova’s (2015) New General Service List (new-GSL); and Browne’s (2014) New General Service List (NGSL). This was done in order to determine which general service list, when combined with the CSAVL/CSAVL-S, would result in the highest total coverage and greatest efficiency. It was found that the combination of Brezina and Gablasova’s (2015) new-GSL with the CSAVL/CSAVL-S was able to cover $94.77 \%$ of the CS corpus, coming within $0.23 \%$ of the lexical threshold. The newGSL/CSAVL/CSAVL-S combination was demonstrated to provide comparable coverage of a CS corpus to that of the GSL/AWL/CSWL combination, but did so with less than half the number of lemmas. 
When combining the CSAVL and CSAVL-S with Browne's (2014) NGSL, it was found that the combination of the NGSL, CSAVL, CSAVL-S, and the NGSL supplemental list was able to exceed the lexical threshold and achieve $95.46 \%$ coverage of the CS corpus. However, the NGSL combination was notably less efficient than the new-GSL/CSAVL/CSAVL-S combination in terms of the total lemma count. For this reason, those educators and learners concerned with list efficiency are recommended to use the CSAVL and CSAVL-S in combination with the new-GSL list.

Though the combination of the CSAVL/CSAVL-S with a general service list was able to reach the 95\% threshold suggested for minimal reading comprehension, you may wonder why a higher target, such as the $98 \%$ threshold recommended by $\mathrm{Hu}$ and Nation (2000) for adequate reading comprehension was not chosen instead. As noted by Hirsch and Nation (1992), there is a relationship of diminishing returns when adding items to a word list; the number of vocabulary items needed to reach coverage of a corpus beyond 95\% increases at an exponential rate. As a consequence of this relationship, the inclusion of additional lemmas to the CSAVL-S supplemental list might allow for total coverage values that could go beyond the 95\% threshold, but it would be at the cost of the list's efficiency. In order to achieve substantial coverage gains in a corpus such as the Computer Science Academic Corpus 2 (CSAC2), it would be necessary to add hundreds of low-frequency lemmas to the CSAVL-S. Many of these low-frequency items could be only representative of the specific corpus that they were extracted from. To demonstrate this, Table 5.1 shows 15 of the most frequent CSAC2 lemmas that were not covered by the new-GSL/CSAVL/CSAVL-S combination. Although the most frequent of these 
lemmas, “silhouette_n”, appeared 112 times in the CSAC2, it only appeared nine times in the Computer Science Academic Corpus 1 (CSAC1). This indicates that this lemma and many of remaining uncovered items of the CSAC2 ("basestation_n”, “furniture_n”, “pinlight_n”) may have been idiosyncratic to that corpus and not representative of wider academic CS vocabulary. Of the lemmas listed in Table 5.1, only “copy_v” and "tag_n” (which both have corresponding ODOCS entries) might be considered useful additions.

Table 5.1 The 15 most frequent CSAC2 lemmas not covered by the newGSL/CSAVL/CSAVL-S combination

\begin{tabular}{lrr}
\hline lemma & CSAC2 freq. & CSAC1 freq. \\
\hline silhouette_n & 112 & 9 \\
basestation_n & 109 & 0 \\
copy_v & 100 & 301 \\
furniture_n & 92 & 20 \\
pinlight_n & 88 & 0 \\
rabbit_n & 87 & 16 \\
tag_n & 75 & 260 \\
aperture_n & 58 & 20 \\
backscatter_n & 57 & 1 \\
coloring_n & 57 & 57 \\
pupil_n & 55 & 41 \\
critique_n & 45 & 13 \\
blendshapes_n & 45 & 18 \\
blacklist_n & 44 & 22 \\
pole_n & 44 & 4 \\
\hline
\end{tabular}

In summary, based on the results of the coverage tests that were reported in the results chapter, it can be said that the CSAVL word list and the combination of the CSAVL/CSAVL-S word lists can provide more efficient coverage of an academic computer science corpus than the currently available word lists that were designed for similar purposes. Additionally, the combination of the new-GSL, CSAVL, and CSAVL-S 
should provide a more direct route than the currently available alternative lists to reaching a minimal level of reading comprehension of academic CS texts.

\subsection{Representativeness and technicality of the CSAVL and CSAVL-S}

The analyses performed in Sections 4.4 and 4.5 of the results chapter were focused on the representativeness and technicality of the CSAVL and CSAVL-S. The term representativeness is used here to refer to whether or not the word list represents the vocabulary found in the target population of texts (i.e. can the contents of a word list be found more often in academic CS texts than other kinds of texts?). The use of the term technicality follows Nation's (2016) definition of technical vocabulary as terms “closely related to the ideas covered in a particular subject area” (p.146). By performing coverage tests on several types of corpora (a general academic corpus, a general English corpus, and a second academic computer science corpus) and making comparisons with technical dictionaries, it was demonstrated that the CSAVL and CSAVL-S are representative of the vocabulary of academic computer science texts and are also specialist lists containing large portions of technical vocabulary.

In Section 4.4, the coverage comparisons of the British National Corpus (BNC) academic and non-academic portions demonstrated that both the CSAVL and CSAVL-S have a higher coverage in an academic corpus than a non-academic one, indicating that these lists are representative of academic vocabulary. Additionally, the word lists covered 
a substantially larger percentage of two CS-academic corpora, the Computer Science Academic Corpus 1 and 2 (the CSAC1 and CSAC2), than they did of the BNC academic corpus, which demonstrated that the content of these word lists are likely to be specifically representative of the written lexicon of academic computer science. Furthermore, the similar coverage of the CSAVL and CSAVL-S of both the CSAC1 and CSAC2 corpora indicates that the contents of these word lists are representative of academic CS vocabulary outside of the source corpus (the CSAC1) and are not just items specific to a single academic CS corpus.

In addition to being representative of the language of academic CS, The CSAVL and CSAVL-S were also shown to contain technical vocabulary. The notion of word technicality was addressed in the 2001 edition of Paul Nation's Learning Vocabulary in Another Language. In the 2001 edition, he estimated that technical vocabulary makes up $5 \%$ of the words in a specialist text. However, in the revised 2013 edition, Nation provides a more nuanced definition of technical vocabulary, which explicitly points out that technical vocabulary can appear in any of the high-, mid-, or low-frequency levels of vocabulary. Nation (2013) gives a new increased estimate of 20-30\% technical words in a specialist text (pp.20). By including numerous high-frequency lemmas that have CSspecific meanings, the CSAVL and CSAVL-S are reflective of Nation's revised definition of technical vocabulary that acknowledges that technical vocabulary can be found at all word frequency levels.

In Section 4.5, the comparisons with the Oxford Dictionary of Computer Science (ODOCS) and Oxford Concise Dictionary of Mathematics (ODOM) showed that 52\% of 
the CSAVL lemmas and $41 \%$ of the CSAVL-S appeared as technical dictionary entries, indicating that these lists include a large portion of technical vocabulary. Minshall, when creating the Computer Science Word List (CSWL), omitted technical terms that also appeared on the GSL and AWL, such as “server_n” and “tree_n”, which limited the number of technical items that were included in the CSWL. When the CSWL, a list that was determined by Minshall to be technical in nature, was examined in this study, it was found that only $26 \%$ of the CSWL lemmas were technical dictionary entries. This provides evidence that the CSAVL and CSAVL-S both contain larger portions of technical vocabulary than the CSWL technical vocabulary list, most likely due to their inclusion of high-frequency words that also had CS-specific meanings.

\subsection{The content of the CSAVL and CSAVL-S}

In Section 4.6, a comparison was made between the contents of six word lists: the new-GSL/CSAVL/CSAVL-S and the GSL/AWL/CSWL. It was found that CSAVL and CSAVL-S word lists are mainly composed of lemmas that also appear in the GSL, AWL, or CSWL. The large portions of Academic Word List (AWL) and Computer Science Word List (CSWL) content in the CSAVL/CSAVL-S is not surprising for a pair of lists that were intended to represent the vocabulary of academic computer science, but the presence of GSL items on the CSAVL/CSAVL-S may be unexpected to those who are unfamiliar with the design of the CSAVL/CSAVL-S. By design, the CSAVL and 
CSAVL-S included high-frequency items that either: 1) have CS-specific meanings (such as “mouse_n”, “tree_n”, and “library_n”); or 2) are more frequently found in an academic CS corpus than a general English corpus (such as “one_n” and “zero_n”). The inclusion of high-frequency items with CS-specific meanings was an intentional part of the CSAVL's design because it allows for a list which can direct English language learners (ELLs) toward noticing differences in use that are context-based. For this reason, the CSAVL items which were found to be technical dictionary entries have been marked (with “*” for CS-specific meanings or “\#” for Mathematics-specific meanings) in the version of the word lists provided in Appendices A and B. By allowing high-frequency items such as “memory_n”, “server_n” and “string_n” into the CSAVL and explicitly marking these items as having CS-specific meanings, the CSAVL describes CS vocabulary in a more detailed and context-specific way than the AWL or CSWL.

In Sections 4.7 of the results chapter, it was demonstrated that the CSAVL and CSAVL-S word lists are composed of the GSL/AWL/CSWL lemmas that are highly representative of the vocabulary of academic computer science. This was done by performing a series of coverage tests on the CSAC2 corpus using two portions of the GSL, AWL, and CSWL word lists: the portions that overlapped with newGSL/CSAVL/CSAVL-S lemmas, and the portions that did not. The results of these tests showed that contents of the GSL/AWL/CSWL that are not included in the newGSL/CSAVL/CSAVL combination have little representation in academic CS texts. The limited relevance of the majority of the content in the GSL/AWL/CSWL to CS-specific texts points out these lists' inefficiency as tools for the development of academic CS 
reading comprehension. The inefficiency of the GSL/AWL/CSWL in a CS context can be linked to their use of the word family counting unit. CSWL word family headwords such as "bug” and "chip” also include the lemmas "bug_v” and “chip_v”, which have little or no representation in the CSAC1 or CSAC2 corpora. The GSL/AWL/CSWL provides no indication of the presence of CS-specific meanings or any indication of which parts of speech or derivational forms are most frequent in CS contexts, leaving list users with the responsibility of performing their own research or making guesses as to which meanings and forms are relevant to the context of academic CS. Since the content of the CSAVL and CSAVL-S was determined at the lemma-level, parts of speech and derivational forms which appeared less frequently in an academic CS context were not included. This resulted in a list that describes the register in a more detailed and context-specific way which might allow learners to more quickly reach adequate reading comprehension in an

academic CS context. Using the CSAVL and CSAVL-S, learners can focus their attention on learning lemmas such as "handle_v" (as in "handle an exception”) rather than other parts of speech which are less related to the context of academic computer science, such as “handle_n”.

\subsection{Usage of the CSAVL and CSAVL-S}

Based on the comparisons and analyses performed in this study, the combination of the CSAVL and CSAVL-S can be considered a more efficient tool than the AVL 
(Gardner \& Davies, 2014) or the pairing of the AWL (Coxhead, 2000) and the CSWL (Minshall, 2013) for developing academic computer science reading comprehension. The CSAVL and CSAVL-S are specifically representative of the vocabulary of academic computer science, contain a large portion of vocabulary that have CS-specific meanings, and provide an indication of which parts of speech and derivational forms are most relevant to a CS context. By comparison, the AVL and the combined AWL/CSWL word lists are notably less efficient in their coverage of an academic CS corpus, provide no indication of CS-specific meanings, and provide no indication of which parts of speech and derivational forms are relevant to academic CS texts. Given these differences with the AVL, AWL, and CSWL, the CSAVL and CSAVL-S word lists are likely to better serve the needs of English language learners seeking the most direct route to English reading comprehension of academic CS texts by providing learners with an improved cost/benefit relationship and by describing the vocabulary of academic CS in a more detailed and context-specific manner. These lists can also serve as a valuable tool for English for Specific Purposes (ESP) course and material designers who need to identify the most frequently used terms that are specifically representative of academic CS in order to maximize the benefits of language instruction within a constrained period of time.

The remainder of this section provides recommendations for the use of the CSAVL and CSAVL-S word lists and suggestions for their practical applications. The following four topics are addressed: 1) The usage of the CSAVL/CSAVL-S in relation to technical 
dictionaries; 2) Suggested uses for educators and pedagogical materials designers; 3) Suggested uses for independent learners; and 4) The limitations for the lists' usages.

1. The usage of the CSAVL/CSAVL-S in relation to technical dictionaries

As noted by Lei and Liu (2016), educators and learners may wonder what benefit exists in studying a specialist technical word list instead of a technical dictionary such as the Oxford Dictionary of Computer Science (ODOCS). The primary reasons to adopt the use of the CSAVL and CSAVL-S over a technical dictionary are size and specificity. The combined CSAVL/CSAVL-S contains a total of 1,606 lemmas, whereas the ODOCS contains 6,479 entries with no information regarding part of speech, word frequency, or distribution. The CSAVL/CSAVL-S items (listed in Appendix A and B) specify part of speech and are ranked by their frequency. Each list represents a different tier of word distribution, the CSAVL being the most evenly distributed words, and the CSAVL-S listing the less evenly distributed ones. This information should help list users facing time constraints decide which items to focus on and in which order; a benefit not available when using only the ODOCS. However, because the CSAVL/CSAVL-S do not provide definitions for terms that they list, they should not be used as replacements for a technical dictionary. Users of the CSAVL and CSAVL-S should also make use of technical dictionaries such as ODOCS and the Oxford Concise Dictionary of Mathematics (ODOM) in conjunction with the word lists. To guide users of the lists toward the appropriate technical dictionary definitions, the items in the CSAVL/CSAVL-S are listed 
with a "*” to denote items that have ODOCS entries, and with a "\#” to denote items that appear in the ODOM.

The decision to explicitly identify the CSAVL/CSAVL-S items with disciplinespecific meanings was informed by Lam (2001), which examined "sub-technical” vocabulary (words with both CS-specific meanings and general high-frequency meanings) and the difficulties that these terms presented for learners. The researcher noted that (exclusively) technical vocabulary items create fewer obstacles for learners than "items of vocabulary from normal English operating within a science context" (pp.28). This would suggest that CSAVL/CSAVL-S items with both technical and general meanings such as “pipe_n”, “bug_n”, or “mouse_n” may create more difficulties for learners than technical domain-specific terms such as "botnet_n”. For this reason, list users should make special note of the terms that have been marked as having disciplinespecific meanings, and consult a technical dictionary in order to find the meaning that is most appropriate for a CS context.

2. Suggested uses for educators and pedagogical material designers

The CSAVL/CSAVL-S can serve as a tool used by educators in the design of an English for Specific Purposes (ESP) course for students intending to study computer science, or by pedagogical material designers to create learning materials for students who have the intention of improving their reading comprehension of English academic CS texts. One of the primary benefits of using corpus-derived, frequency-based word lists such as the CSAVL and CSAVL-S is that they allow educators to make decisions 
regarding which words should be chosen for study (and in which order) based on data derived from collections of actual language usage. Educators using the CSAVL/CSAVLS should prioritize the lemmas in order of their ranked frequency. The versions of the lists provided in Appendices A and B include a rank number to the left of each item. Items with the lowest rank numbers were the most frequent items in the academic CS corpus that the lists were extracted from, and should be the first priorities for learners to acquire. In selecting terms for course or material design, users of the CSAVL and CSAVL-S lists should first focus on the 904 lemmas of the CSAVL, which were found to be the most frequent and evenly distributed terms in the corpus of academic CS texts. The 702 lemmas of the CSAVL-S, which were less frequent and were clustered in specific CS sub-disciplines, such as hardware or mathematics, should be turned to only after the main CSAVL list has been studied.

Educators and learning material designers can teach list items directly and provide explicit definitions, but should also situate the terms listed in the CSAVL/CSAVL-S in texts that contextualize the CS-specific meanings of these words. This can be accomplished by providing concordance lines produced by searches in digitized CS textbooks, the Association of Computing Machinery’s (ACM) digital library of CS journal articles, or if available, a corpus of academic CS texts. When teaching CSAVL lemmas with discipline-specific meanings, learners should be introduced to both their general-usage meanings and their discipline specific meanings. In comparisons of the general-usage meanings with the discipline-specific meanings, educators can introduce concepts such as metaphorical extension in order to point out the connections between 
the multiple meanings of these polysemous terms. Terms with CS-specific meanings should be taught using examples of actual academic CS texts that situate the terms in context in order to aid learners in noticing and identifying contextual and metatextual clues that index the writer's intended meanings for each term. When selecting academic CS texts for this purpose, educators should take care to avoid texts that use the terms in lexically ambiguous ways, and instead select texts which clearly demonstrate the discipline-specific meanings of the term.

\section{Suggested uses for independent learners}

English language learners preparing for study in computer science can use the CSAVL/CSAVL-S to identify gaps in their own CS vocabulary knowledge and to set vocabulary goals for independent study. As with the suggestions provided for educators, learners should prioritize the lemmas of CSAVL before those of the CSAVL-S, and prioritize lemmas in each list based on their ranked order. When studying the CSAVL/CSAVL-S items that have discipline-specific meanings (listed with an “*” or “\#” symbol), learners should consult the appropriate technical dictionary in order to find the discipline-specific meanings for these terms and compare them to their definitions in general-usage dictionaries.

For more proficient independent learners that already are familiar with a range of vocabulary similar to that which can be found on the GSL and AWL word lists, the primary focus when studying the CSAVL/CSAVL-S lists should be placed on the terms that are marked as having discipline-specific meanings and learning how those meanings 
differ from the term's general usage meanings. Understanding how the terms are used within an academic CS context is also vital for independent learners. These learners should also make use of searches in digitized CS textbooks or the Association of Computing Machinery's (ACM) digital library to find examples of the terms' usages within CS texts and contextualize the CS-specific meanings of these words.

\section{Limitations for the usages of the CSAVL/CSAVL-S}

The CSAVL/CSAVL-S were designed specifically for the purpose of developing reading comprehension of academic computer science texts. While this narrow focus allows the lists to efficiently serve their purpose, it also limits the range of their usages. The CSAVL/CSAVL-S lists were derived from a discipline-specific corpus, and consequently, are not likely to be ideal tools for helping learners develop more generalized forms of reading proficiency. Similarly, since the CSAVL/CSAVL-S lists were derived from a corpus of written texts, they should not be used with the intention of developing spoken English proficiency, nor should they be assumed to represent the vocabulary of spoken English that is produced within the discipline of CS. Furthermore, since the lemmas of the CSAVL/CSAVL-S were drawn from a corpus of academic texts, they should not be assumed to represent the vocabulary of written communication of practitioners within the profession of CS. Although the narrow focus of the CSAVL/CSAVL-S limits their possible uses, for English learners with the specific goal of increasing reading comprehension ability in academic CS texts, the CSAVL/CSAVL-S will likely be a useful set of tools. 


\subsection{The limitations of the CSAVL project}

Although the primary objectives of this study were met, limitations were encountered which should be taken into consideration when evaluating the findings. The first of the limitations was the size of the 3.5-million-word CSAC1 corpus. While the CSAC1 was of similar size to the corpus used for the creation of the AWL, the CSAC1 could have been expanded in order to include a greater sampling of academic CS texts. This would have allowed for the inclusion of a wider range of textbooks than the 1-2 textbooks that were sampled for each textbook sub-corpus of the CSAC1. A larger and more varied sampling of authors within each sub-corpus would have helped to mitigate any possible issues of author bias within the CSAC1.

A second limitation of the methodology used in this study is that although frequency counts were made of POS-tagged lemmas in the CSAC1, there was no identification made of the intended meanings of the terms in each specific context that they were found. While “memory_n” was one of the most frequent terms in the CSAC1, it was unknown how many of the uses of "memory" were referring its CS-specific meaning and how many of the uses instead referred to its general usage meaning. While the CSAVL inclusion criterion six required that general new-GSL lemmas also had a CS-specific meaning in order to be included, it was ultimately unknown whether the CSAC1 appearances of these words were actually used with those CS meanings. Future word list research may be able to overcome this limitation through the development of semantic 
tagging systems that might be able to better identify intended word meanings in each specific context of use (Rayson et al., 2004; Wilks \& Stevenson, 1997).

A third limitation of the methodology of this study was found in the lemma inclusion criteria for the CSAVL-S. When creating the CSAVL-S supplemental list, I removed the range and dispersion criteria in order to include lemmas that were more specific to academic CS sub-disciplines such as hardware or networking. While this resulted in the desired effect of including lemmas that were clustered in specific sub-disciplines, it also removed safeguards that prevented the inclusion of lemmas that were used repetitively within a single text. Due to a single textbook chapter that repeatedly used the word “rabbit” (to describe an algorithm that calculates the reproduction of rabbits over time), the lemma "rabbit_n” was initially found in the CSAVL-S and later manually removed. I advise any word list makers attempting to reproduce the methodology of the CSAVL-S to also include a criterion that accounts for the repetitive occurrence of lemmas within a single text.

Finally, a limitation of any discipline-specific list including technical terms is that teachers who make use of the CSAVL should have some preexisting background knowledge of either CS or Mathematics. Instructors without knowledge of the CS concepts that are related to these terms should make efforts to educate themselves especially regarding the CS-specific meanings of the CSAVL terms that they will be 
teaching.

\subsection{Possibilities for future corpus and word list research}

In addition to the limitations, several complications noted during this project pointed toward possibilities for additional research and word list tool development. In this section, I present the following suggestions for avenues of future research: 1) A standardized set of part-of-speech (POS) groupings; 2) Improvements to Lancsbox lemmatization and POS tagging; 3) A tool for converting lemmas to types; 4) A tool for performing lemma-based coverage tests; 5) An expanded academic CS multi-word and collocate list; and 6) An investigation into the effects of the CSAC1 corpus design on the CSAVL.

1. A standardized set of POS groupings

One of these complications was that POS-tagged lemmas were found to be inconsistently grouped by different researchers and corpus software designers. For example, in the default settings of the Lancsbox software (Brezina et al., 2018), which was used to create the CSAVL, quantifiers (such as "many”) were grouped as adjectives and labeled as “many_adj”. However, in the new-GSL word list (also in part developed by Brezina), quantifiers were instead placed in the category of " $\mathrm{x}$ " along with determiners and particles, and the quantifier "many" was labeled as "many_x" rather than 
“many_adj”. The variations in these POS groupings should be noted by those attempting to reproduce POS-tagged lemma research, since different quantitative results may be found from a single corpus that has been POS-tagged using a different grouping method.

\section{Improvements to Lancsbox lemmatization and POS tagging}

The POS tagger used in this project (included with the Lancsbox software), Treetagger (Schmid, 1994, 1995), while generally producing accurate results, was found to incorrectly tag imperative verbs in sentence initial position (“Assume $\mathrm{X}$ has a value of...”) as nouns. Imperative structures are frequent in textbooks and this error in tagging could have led to misrepresentations in the corpus had they not been found. Additionally, nouns were sometimes separated into singular and plural forms (“system_n” and “systems_n” were separately listed). These issues required that manual corrections be made when preparing data for both lemma extraction (described in Section 3.10-11) and before word list coverage tests. This correction process may have been avoidable if an entirely different software tool had been selected or the tagger had been interacted with directly instead of through the Lancsbox interface, which limited control over the TreeTagger options.

\section{A tool for converting lemmas to types}

The task of converting lists to different units of counting also pointed out a need for software that is able to convert POS-tagged lemmas to types accurately. The timeconsuming process of converting lemmas to types involved expanding lemmas to all 
inflectional forms and then manually removing forms that did not match the original lemma’s part of speech (this process was described in Section 4.1). If a POS-tagged lemma dictionary were created, a piece of software similar to the Familizer + Lemmatizer (Cobb, n.d.) would be simple to implement.

4. A tool for performing lemma-based coverage tests

In addition to a lemma-to-type conversion tool, software that can perform POStagged lemma coverage checks of corpora would be a useful resource for future word list makers. Currently, the Compleat Lexical Tutor site (Cobb, n.d.), AntWordProfiler (Anthony, 2008), and Range (Nation \& Heatley, 2002) are the most accessible tools available for performing coverage checks, but all three of these tools are designed to perform type-based tests only. POS-tagged lemma coverage tests require coding ability to implement, making the creation of lists of this type inaccessible to many language educators. A lemma-based coverage tester could be a useful addition to the Lancsbox software package, or part of a new program with a similarly user-friendly graphical interface.

5. An expanded academic CS multi-word and collocate list

Another complication that pointed toward future research came from the hyphenated forms in the CSAC1 and CSAC2 corpora. As described in Section 3.8, the removal of numbers from the CSAC1 source corpus resulted in the presence of hyphenated forms such as "real-time”, but also a large number of split forms such as “-dimensional” and “- 
bit”. In order to eliminate this inconsistency, all hyphens were removed from the corpora before tokenization. However, the decision to remove hyphens also meant that multiword hyphenated forms such as "real-time" were not included in the final versions of the CSAVL/CSAVL-S. When creating the Computer Science Word List (CSWL), Minshall (2013) also provided a somewhat limited 23-item Computer Science Multi-Word List (CSMWL). A more comprehensive multi-word CS vocabulary list that also includes hyphenated forms and a list of common collocations may be a useful project for future research and could provide learners with an additional level of description of the language of academic CS.

6. An investigation into the effects of the CSAC1 corpus design on the CSAVL

By expanding the size of the CSAC1, or by including a greater variety of text types, it may be possible to investigate what effects specific design choices have on the resulting word lists. Experiments with an increased corpus size could lead to a determination of the number of articles and textbooks required to reach lexical saturation, the point where adding additional texts will add few if any new lemmas to the corpus (Miller \& Biber, 2015). The addition of other types of academic CS texts, such as course materials (slides, handouts, assignments, and lab manuals) would also likely affect the lemmas of the resulting word lists and could produce a list that is more representative of the full variety of academic reading types that CS students might encounter. 


\subsection{Conclusion}

Though academic vocabulary knowledge has been described as being vital to both reading comprehension (Corson, 1997; Jacobs, 2008; Nagy \& Townsend, 2012) and academic success (Goldenberg, 2008), it has been only recently that researchers such as Lei and Liu (2016) have begun to develop discipline-specific academic vocabulary lists that take these three principles into account: 1) that academic and other specialist vocabulary can be found at all levels of word frequency; 2) that for many vocabulary items, certain derivational forms and parts of speech are more relevant than others to specific disciplines; and 3) that many polysemous vocabulary items have both generalusage meanings and meanings that are associated with specific disciplines.

These principals guided the development of the CSAVL project, which produced a discipline-specific computer science academic word list that built upon the methods of Gardner and Davies (2014) and Lei and Liu (2016). By allowing for the inclusion of high-frequency words with discipline-specific meanings (such as “mouse_n” and “tree_n”) into the word list, the CSAVL more comprehensively represents the vocabulary related to the concepts and practices of computer science than previous lists which restricted general high-frequency items, such as the Computer Science Word List (CSWL) (Minshall, 2013). By explicitly indicating which terms have CS-specific meanings, and which parts of speech and derivational forms are most relevant to CS, the CSAVL reduces the burden of interpreting word list contents on list users and describes the vocabulary of CS more efficiently and in greater detail than was possible through 
previous methods. It is my hope that learners will be able to benefit from the efficiency and detail of the CSAVL, and that word list developers will be able to take advantage of and improve on the methods of this study when producing their own specialist word lists. 


\section{References}

Anthony, L. (2008). AntWordProfiler [Computer software]. Retrieved December 1st, 2019, from http://www.antlab.sci.waseda.ac.jp/software.html

Atkins, S. (1992). Corpus design criteria. Literary and Linguistic Computing, 7(1), 1-16. https://doi.org/10.1093/llc/7.1.1

Bauer, L., \& Nation, I. S. P. (1993). Word families. International Journal of Lexicography, 6(3), 253-279.

Baumann, J. F., \& Graves, M. F. (2010). What Is Academic Vocabulary? Journal of Adolescent \& Adult Literacy, 54(1), 4-12.

Beck, I. L., McKeown, M. G., \& Kucan, L. (2013). Bringing words to life: Robust vocabulary instruction. Guilford Press.

Berger, E. D. (2016). CSRankings. Retrieved November 7, 2019, from https://csrankings.org

Biber, D. (1989). A typology of English texts. Linguistics, 27, 3-43.

Biber, D. (1993). Representativeness in corpus design. Literary and Linguistic Computing, 8(4), 15.

Biemiller, A. (2010). Words worth teaching: Closing the vocabulary gap. Columbus, OH: McGraw-Hill SRA.

Brezina, V., \& Gablasova, D. (2015). Is there a core general vocabulary? Introducing the new general service list. Applied Linguistics, 36(1), 1-22. https://doi.org/10.1093/applin/amt018 
Brezina, V., McEnery, T. \& Wattam, S. (2015). Collocations in context: A new perspective on collocation networks. International Journal of Corpus Linguistics, 20(2), 139-173.

Brezina, V., Timperley, M., \& McEnery, A. (2018). \#LancsBox v. 4.x. [software package].

Browne, C. (2014). A new general service list: The better mousetrap we've been looking for? Vocabulary Learning and Instruction, 3(2), 1-10. https://doi.org/10.7820/vli.v03.2

Butterfield, A., Ngondi, G. E., \& Kerr, A. (Eds.). (2016). A dictionary of computer science. Oxford University Press.

Campion, M. E., \& Elley, W. B. (1971). An academic vocabulary list. New Zealand Council for Educational Research.

Carter, R. (2012). Vocabulary: Applied linguistic perspectives. Routledge.

Chung, T. M., \& Nation, P. (2004). Identifying technical vocabulary. System, 32(2), 251263. https://doi.org/10.1016/j.system.2003.11.008

Clancy, B. (2010). Building a corpus to represent a variety of a language. In A. O’Keeffe \& M. McCarthy (Eds.), The Routledge handbook of corpus linguistics (pp. 108120). Routledge. https://doi.org/10.4324/9780203856949-15

Clapham, C., \& Nicholson, J. (2009). The concise Oxford dictionary of mathematics. Oxford University Press.

Cobb, T., Familizer + Lemmatizer v2.2 [computer program]. Accessed 01 Dec 2019 at https://www.lextutor.ca/familizer/. 
Cohen, A., Glasman, H., Rosenbaum-Cohen, P. R., \& Ferrara, J. (1979). Reading English for specialized purposes: Discourse analysis and the use of student informants. TESOL Quarterly, 13(4), 551-564.

Conrad, S. M. (1996). Investigating academic texts with corpus-based techniques: An example from biology. Linguistics and Education, 8(3), 299-326. https://doi.org/10.1016/S0898-5898(96)90025-X

Corson, D. (1997). The learning and use of academic English words. Language Learning, 47(4), 671-718. https://doi.org/10.1111/0023-8333.00025

Coxhead, A. (2000). A new academic word list. TESOL Quarterly, 34(2), 213-238. https://doi.org/10.2307/3587951

Coxhead, A. (2016). Reflecting on Coxhead (2000), “A new academic word list.” TESOL Quarterly, 50(1), 181-185. https://doi.org/10.1002/tesq.287

Coxhead, A., \& Hirsch, D. (2007). A pilot science-specific word list. Revue Française de Linguistique Appliquée, 12(2), 65. https://doi.org/10.3917/rfla.122.0065

Dang, T. N. Y., \& Webb, S. (2016). Making an essential word list for beginners. In I. S. P. Nation (Ed.), Making and using word lists for language learning and testing (pp. 153-167). John Benjamins Publishing Company. https://doi.org/10.1075/z.208

Davies, M. (2008). The corpus of contemporary American English. Available at: http://corpus.byu.edu/coca/.

Elley, W. B. (1989). Vocabulary acquisition from listening to stories. Reading Research Quarterly, 24(2), 174. https://doi.org/10.2307/747863 
Francis, W. N., Kučera, H., \& Mackie, A. W. (1982). Frequency analysis of English usage: Lexicon and grammar. Houghton Mifflin Harcourt (HMH).

Francis, W. N., \& Kučera, H. (1967). A computational analysis of present-day American English. Providence, RI: Brown University Press.

Fraser, S. (2007). Providing ESP learners with the vocabulary they need: Corpora and the creation of specialized word lists. Hiroshima Studies in Language and Language Education, 10, 127-143.

Gardner, D., \& Davies, M. (2014). A new academic vocabulary list. Applied Linguistics, 35(3), 305-327. https://doi.org/10.1093/applin/amt015

Ghadessy, P. (1979). Frequency counts, word lists, and materials preparation: A new approach. English Teaching Forum, 17, 24-27.

Gilner, L. (2011). A primer on the General Service List. Reading in a Foreign Language, 23(1), 65-83.

Gries, S. Th. (2019). Analyzing dispersion. In S. Th. Gries \& M. Paquot (Eds.), Practical Handbook of Corpus Linguistics. Retrieved from http://www.stgries.info/research/ToApp_STG_Dispersion_PHCL.pdf

Gardner, D. (2007). Validating the construct of word in applied corpus-based vocabulary research: A critical survey. Applied Linguistics, 28(2), 241-265.

https://doi.org/10.1093/applin/amm010

Gardner, D. (2013). Exploring vocabulary: Language in action. Routledge.

Goldenberg, C. (2008). Teaching English Language Learners: What the research doesand does not-say. American Educator, Summer, 8-44. 
Graves, M. F. (2016). The vocabulary book: Learning and instruction. Teachers College Press.

Guo, P. J. (2018). Non-native English speakers learning computer programming: Barriers, desires, and design opportunities. Proceedings of the 2018 CHI Conference on Human Factors in Computing Systems - CHI ’18, 1-14. https://doi.org/10.1145/3173574.3173970

Hirsh, D. \& Nation, I. S. P. (1992). What vocabulary size is needed to read unsimplified texts for pleasure? Reading in a Foreign Language, 8, 689-696.

Hyland, K., \& Tse, P. (2007). Is there an “academic vocabulary”? TESOL Quarterly, 41(2), 235-253. https://doi.org/10.1002/j.1545-7249.2007.tb00058.x

Hsia, S., Chung, P. K., \& Wong, D. (1995). ESL learners’ word organization strategies: A case of Chinese learners of English words in Hong Kong. Language and Education, 9(2), 81-102.

Hsu, W. (2011). A business word list for prospective EFL business postgraduates. The Asian ESP Journal, 7(4), 63-99.

Hsu, W. (2013). Bridging the vocabulary gap for EFL medical undergraduates: The establishment of a medical word list. Language Teaching Research, 17(4), 454-484. https://doi.org/10.1177/1362168813494121

Hsu, W. (2014). Measuring the vocabulary load of engineering textbooks for EFL undergraduates. English for Specific Purposes, 33, 54-65. https://doi.org/10.1016/j.esp.2013.07.001 
Hu, M., \& Nation, I. S. P. (2000). Unknown vocabulary density and reading comprehension. Reading in a Foreign Language, 13(1), 403-430.

Jacobs, V. (2008). Adolescent literacy: Putting the crisis in context. Harvard Educational Review, 78(1), 7-39. https://doi.org/10.17763/haer.78.1.c577751kq7803857

Jenkins, J. R., Stein, M. L., \& Wysocki, K. (1984). Learning vocabulary through reading. American Educational Research Journal, 21(4), 767-787.

Khani, R., \& Tazik, K. (2013). Towards the development of an academic word list for Applied Linguistics research articles. RELC Journal, 44(2), 209-232. https://doi.org/10.1177/0033688213488432

Konstantakis, N. (2007). Creating a business word list for teaching business English. ELIA, 7, 79-102.

Lam, J. (2001). A study of semi-technical vocabulary in computer science texts with special reference to ESP teaching and lexicography. In G. James (Ed.), Research reports (Vol. 3). Language Center, Hong Kong University of Science and Technology.

Laufer, B. (1989). What percentage of text-lexis is essential for comprehension? In C. Lauren \& M. Nordman (Eds.), Special language: From humans to thinking machines (pp. 316-323).

Laufer, B., \& Ravenhorst-Kalovski, G. C. (2010). Lexical threshold revisited: Lexical text coverage, learners’ vocabulary size and reading comprehension. Reading in a Foreign Language, 22(1), 15-30. 
Lei, L., \& Liu, D. (2016). A new medical academic word list: A corpus-based study with enhanced methodology. Journal of English for Academic Purposes, 22, 42-53. https://doi.org/10.1016/j.jeap.2016.01.008

Lynn, R. W. (1973). Preparing word-lists: A suggested method. RELC Journal, 4(1), 2528. https://doi.org/ 10.1177/003368827300400103

Martínez, I. A., Beck, S. C., \& Panza, C. B. (2009). Academic vocabulary in agriculture research articles: A corpus-based study. English for Specific Purposes, 28(3), 183198. https://doi.org/10.1016/j.esp.2009.04.003

Maylath, B. A. R. (1996). Words make a difference: The effects of Greco-Latinate and Anglo-Saxon lexical variation on college writing instructors. Research in the Teaching of English, 30(2), 220-247.

Maylath, B. A. R. (2013). Assessor's language awareness in the evaluation of academic writing. In L. van Lier \& D. Corson (Eds.), Knowledge about language. Boston: Kluwer.

Micher, J. C. (2012). A Method for Correcting Broken Hyphenations in Noisy English Text. Army Research Laboratory. https://doi.org/10.21236/ADA561948

Mikheev, A. (2014). Text Segmentation. The Oxford handbook of computational linguistics 2nd edition. https://doi.org/10.1093/oxfordhb/9780199573691.013.34

Miller, D., \& Biber, D. (2015). Evaluating reliability in quantitative vocabulary studies: The influence of corpus design and composition. International Journal of Corpus Linguistics, 20(1), 30-53. https://doi.org/10.1075/ijcl.20.1.02mil 
Minshall, D. E. (2013). A computer science word list (Master's thesis). Retrieved from https://www.baleap.org/wp-content/uploads/2016/03/Daniel-Minshall.pdf

Nagy, W. E., \& Anderson, R. C. (1984). How many words are there in printed school English?. Reading Research Quarterly, 19(3), 304-330.

Nagy, W., \& Townsend, D. (2012). Words as tools: Learning academic vocabulary as language acquisition. Reading Research Quarterly, 47(1), 91-108. https://doi.org/10.1002/RRQ.011

Nation, I. S. P. (2001). Learning vocabulary in another language. Cambridge ; New York: Cambridge University Press.

Nation, I. S. P. (2008). Teaching vocabulary: Strategies and techniques. Boston, MA: Heinlen Cengage Learning.

Nation, I. S. P. (2013). Learning vocabulary in another language ( $2^{\text {nd }}$ ed.). Cambridge ; New York: Cambridge University Press.

Nation, I. S. P. (2016). Making and using word lists for language learning and testing (3rd ed.). Amsterdam, Netherlands: John Benjamins Publishing Company.

Nation, I.S.P., \& Heatley, A. (2002). Range [Computer software]. Retrieved July 6th, 2019, from http://www.victoria.ac.nz/lals/about/staff/paul-nation

Nation, I.S.P. \& Sorell, J. (2016). Corpus selection and design. In I.S.P. Nation (Ed.), Making and using word lists for language learning and testing (pp.95-105). Johns Benjamins Publishing Company. 
Nation, I. S. P., \& Waring, R. (1997). Vocabulary size, text coverage and word lists. In I. Schmitt \& M. McCarthy (Eds.), Vocabulary: Description, acquisition and pedagogy (pp. 6-19).

National Foundation for American Policy. (2017). The importance of international students to American science and engineering [NFAP policy brief]. Retrieved from http://nfap.com/wp-content/uploads/2017/10/The-Importance-of-InternationalStudents.NFAP-Policy-Brief.October-20171.pdf

Nippold, M.A. (2007). Later language development: School-age children, adolescents, and young adults (3rd ed.). Austin, TX: Pro-Ed.

Oakes, M. P., \& Farrow, M. (2007). Use of the chi-squared test to examine vocabulary differences in English language corpora representing seven different countries. Literary and Linguistic Computing, 22(1), 85-99. https://doi.org/10.1093/llc/fql044

Praninskas, J. (1972). American university word list. London: Longman.

Quero, B. (2015). Estimating the vocabulary size of L1 Spanish ESP learners and the vocabulary load of medical textbooks. Unpublished PhD Thesis, Victoria University of Wellington, New Zealand.

Rayson, P., Archer, D., Piao, S., \& McEnery, A. M. (2004). The UCREL semantic analysis system. In Proceedings of the beyond named entity recognition semantic labelling for NLP tasks workshop, Lisbon, Portugal, 2004 (pp. 7-12).

Reppen, R. (2010). Building a corpus. In A. O’Keeffe \& M. McCarthy (Eds.), The Routledge handbook of corpus linguistics (pp. 31-37). Routledge. https://doi.org/10.4324/9780203856949-15 
Savický, P., \& Hlavácová, J. (2002). Measures of word commonness. Journal of Quantitative Linguistics, 9(3), 215-231. https://doi.org/10.1076/jqul.9.3.215.14124

Schloegl, C., \& Gorraiz, J. (2011). Global usage versus global citation metrics: The case of pharmacology journals. Journal of the American Society for Information Science and Technology, 62(1), 161-170.

Schmid, H. (1994). Probabilistic part-of-speech tagging using decision trees. Proceedings of the International Conference on New Methods in Language Processing. Manchester, UK.

Schmid, H. (1995). Improvements in part-of-speech tagging with an application to German. Proceedings of the ACL SIGDAT-Workshop. Dublin, Ireland.

Scott, M., 2016, WordSmith Tools version 7 [computer program]. Stroud: Lexical Analysis Software.

Sinclair, J. (1991). Corpus, concordance, collocation. Oxford University Press.

Sinclair, J. (2005). Corpus and text-basic principles. Developing linguistic corpora: A guide to good practice (pp. 1-16). Oxbow Books.

Strevens, P. (1973). Technical, technological, and scientific English. ELT Journal, 27, 223-234.

U.S. News \& World Report. (2018). The best computer science programs in America, ranked. Retrieved November 7, 2019, from https://www.usnews.com/best-graduateschools/top-science-schools/computer-science-rankings

Vongpumivitch, V., Huang, J., \& Chang, Y.-C. (2009). Frequency analysis of the words in the Academic Word List (AWL) and non-AWL content words in applied 
linguistics research papers. English for Specific Purposes, 28(1), 33-41. https://doi.org/ 10.1016/j.esp.2008.08.003

Wan, J. K., Hua, P. H., Rousseau, R., \& Sun, X. K. (2010). The journal download immediacy index (DII): experiences using a Chinese full-text database. Scientometrics, 82(3), 555-566.

Wang, J., Liang, S., \& Ge, G. (2008). Establishment of a medical academic word list. English for Specific Purposes, 27(4), 442-458. https://doi.org/ 10.1016/j.esp.2008.05.003

Ward, J. (1999). How large a vocabulary do EAP engineering students need? Reading in a Foreign Language, 12(2), 309-323.

Ward, J. (2009). A basic engineering English word list for less proficient foundation engineering undergraduates. English for Specific Purposes, 28(3), 170-182. https://doi.org/10.1016/j.esp.2009.04.001

Watson, A. B. (2009). Comparing citations and downloads for individual articles at the Journal of Vision. Journal of Vision, 9(4), i-i. https://doi.org/ 10.1167/9.4.i

West, M. (1953). A general service list of English words. London, New York: Longman, Green.

Wilks, Y., \& Stevenson, M. (1997). Sense tagging: Semantic tagging with a lexicon. https://arxiv.org/abs/cmp-lg/9705016v1

Xue, G., \& Nation, I. S. P. (1984). A university word list. Language Learning and Communication, 3(2), 215-229. 
Yang, M.-N. (2015). A nursing academic word list. English for Specific Purposes, 37, 27-38. https://doi.org/ 10.1016/j.esp.2014.05.003

Zimmerman, C. B. (2009). Word knowledge: A vocabulary teacher's handbook. Oxford University Press. 


\section{Appendix A: The Computer Science Academic Vocabulary List (CSAVL)}

\section{Key:}

$\mathrm{n}$ : noun, v: verb, adj: adjective, adv: adverb

*: Computer science-specific meaning (listed in Oxford Dictionary of Computer Science $7^{\text {th }}$ ed.)

\#: Mathematics-specific meaning (listed in The Concise Oxford Dictionary of Mathematics, $4^{\text {th }}$ ed.)

(items found in both dictionaries are only listed with asterisks)

\section{Format:}

(rank) (special meaning) (lemma)

\begin{tabular}{|c|c|c|}
\hline 1 & $*$ & system_n \\
\hline 2 & $*$ & data/datum_n \\
\hline 3 & $*$ & algorithm_n \\
\hline 4 & 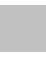 & such_adj \\
\hline 5 & $*$ & base_v \\
\hline 6 & $*$ & node_n \\
\hline 7 & $*$ & model_n \\
\hline 8 & $*$ & case_n \\
\hline 9 & $*$ & program_n \\
\hline 10 & $*$ & information_n \\
\hline 11 & $*$ & set_n \\
\hline 12 & $*$ & code_n \\
\hline 13 & $*$ & function_n \\
\hline 14 & $*$ & process_n \\
\hline 15 & $*$ & application_n \\
\hline 16 & $*$ & software_n \\
\hline 17 & $*$ & class_n \\
\hline 18 & $*$ & point_n \\
\hline 19 & $*$ & type_n \\
\hline 20 & $*$ & network_n \\
\hline 21 & $*$ & tree_n \\
\hline 22 & $*$ & object_n \\
\hline 23 & $*$ & element_n \\
\hline 24 & $*$ & input_n \\
\hline 25 & $*$ & operation_n \\
\hline 26 & $*$ & level_n \\
\hline 27 & $*$ & memory_n \\
\hline 28 & $*$ & table_n \\
\hline 29 & $*$ & order_n \\
\hline 30 & $*$ & file_n \\
\hline 31 & $*$ & variable_n \\
\hline
\end{tabular}

\begin{tabular}{|c|c|c|}
\hline 32 & $*$ & language_n \\
\hline 33 & $*$ & write_v \\
\hline 34 & $*$ & list_n \\
\hline 35 & $*$ & structure_n \\
\hline 36 & $\#$ & compute_v \\
\hline 37 & $*$ & sequence_n \\
\hline 38 & $*$ & computer_n \\
\hline 39 & $*$ & bit_n \\
\hline 40 & $*$ & probability_n \\
\hline 41 & $*$ & machine_n \\
\hline 42 & $*$ & array_n \\
\hline 43 & $*$ & page_n \\
\hline 44 & $*$ & error_n \\
\hline 45 & $*$ & step_n \\
\hline 46 & $*$ & search_n \\
\hline 47 & & most_adj \\
\hline 48 & $*$ & path_n \\
\hline 49 & $*$ & graph_n \\
\hline 50 & $*$ & web_n \\
\hline 51 & $*$ & length_n \\
\hline 52 & & several_adj \\
\hline 53 & $*$ & security_n \\
\hline 54 & $*$ & proof_n \\
\hline 55 & $*$ & access_n \\
\hline 56 & & obtain_v \\
\hline 57 & $*$ & matrix_n \\
\hline 58 & $*$ & task_n \\
\hline 59 & $*$ & image_n \\
\hline 60 & $*$ & form_n \\
\hline 61 & $*$ & return_n \\
\hline 62 & $*$ & interface_n \\
\hline
\end{tabular}




\begin{tabular}{|c|c|}
\hline 63 & * resource_n \\
\hline 64 & * address $\mathrm{n}$ \\
\hline 65 & * implementation_n \\
\hline 66 & * loop_n \\
\hline 67 & * first_adv \\
\hline 68 & * read_v \\
\hline 69 & * location_n \\
\hline 70 & * hardware $n$ \\
\hline 71 & behavior_n \\
\hline 72 & * programming_n \\
\hline 73 & * field_n \\
\hline 74 & * key_n \\
\hline 75 & * parameter_n \\
\hline 76 & * distribution_n \\
\hline 77 & * definition_n \\
\hline 78 & * instance_n \\
\hline 79 & * interaction_n \\
\hline 80 & * internet_n \\
\hline 81 & * representation_n \\
\hline 82 & * edge_n \\
\hline 83 & * stack_n \\
\hline 84 & * return $v$ \\
\hline 85 & * procedure_n \\
\hline 86 & * link_n \\
\hline 87 & * output_n \\
\hline 88 & * block_n \\
\hline 89 & * domain_n \\
\hline 90 & * store_v \\
\hline 91 & * call_n \\
\hline 92 & * device_n \\
\hline 93 & * server_n \\
\hline 94 & * static_adj \\
\hline 95 & dataset_n \\
\hline 96 & detection_n \\
\hline 97 & * write_n \\
\hline 98 & * execute_v \\
\hline 99 & \# least_adj \\
\hline 100 & * key_adj \\
\hline 101 & * range_n \\
\hline 102 & * pattern_n \\
\hline 103 & * vector_n \\
\hline 104 & binary_adj \\
\hline 105 & * constraint_n \\
\hline 106 & * message_n \\
\hline
\end{tabular}

\begin{tabular}{|c|c|}
\hline 107 & * environment_n \\
\hline 108 & * tool $n$ \\
\hline 109 & query_n \\
\hline 110 & * phase_n \\
\hline 111 & * cluster $\mathrm{n}$ \\
\hline 112 & * architecture_n \\
\hline 113 & * root_n \\
\hline 114 & * character_n \\
\hline 115 & * processor_n \\
\hline 116 & due_adj \\
\hline 117 & \# computation_n \\
\hline 118 & * standard_adj \\
\hline 119 & * expression_n \\
\hline 120 & * cell_n \\
\hline 121 & * client_n \\
\hline 122 & * constant_adj \\
\hline 123 & * address_v \\
\hline 124 & * database_n \\
\hline 125 & * access_v \\
\hline 126 & * argument_n \\
\hline 127 & * online_adj \\
\hline 128 & * dynamic_adj \\
\hline 129 & * simulation_n \\
\hline 130 & * construct_v \\
\hline 131 & * degree_n \\
\hline 132 & \# log_n \\
\hline 133 & * layer_n \\
\hline 134 & linear_adj \\
\hline 135 & * select_v \\
\hline 136 & * choice_n \\
\hline 137 & \# optimal_adj \\
\hline 138 & * index_n \\
\hline 139 & * complexity_n \\
\hline 140 & * signal_n \\
\hline 141 & analyze_v \\
\hline 142 & execution_n \\
\hline 143 & specify_v \\
\hline 144 & corresponding_adj \\
\hline 145 & * display_v \\
\hline 146 & * document_n \\
\hline 147 & * model_v \\
\hline 148 & * handle_v \\
\hline 149 & * mode_n \\
\hline 150 & * frame_n \\
\hline
\end{tabular}




\begin{tabular}{|c|c|c|}
\hline 152 & * & mean $n$ \\
\hline 153 & * & equation $n$ \\
\hline 154 & * & scheme $n$ \\
\hline 155 & * & theory_n \\
\hline 156 & * & knowledge $n$ \\
\hline 157 & * & physical adj \\
\hline 158 & * & cycle $n$ \\
\hline 159 & & accord_v \\
\hline 160 & $*$ & optimization_n \\
\hline 161 & * & logic_n \\
\hline 162 & & classification_n \\
\hline 163 & $*$ & cluster_v \\
\hline 164 & * & enable $v$ \\
\hline 165 & & request_n \\
\hline 166 & . & denote_v \\
\hline 167 & $*$ & accuracy_n \\
\hline 168 & * & process $\mathrm{v}$ \\
\hline 169 & & sort_v \\
\hline 170 & & engineering_n \\
\hline 171 & $*$ & operator_n \\
\hline 172 & & formula_n \\
\hline 173 & $*$ & failure_n \\
\hline 174 & $*$ & measure_v \\
\hline 175 & $\#$ & interval_n \\
\hline 176 & & virtual_adj \\
\hline 177 & $*$ & share_v \\
\hline 178 & & assign_v \\
\hline 179 & $*$ & symbol_n \\
\hline 180 & $*$ & framework_n \\
\hline 181 & $*$ & exception_n \\
\hline 182 & * & testing_n \\
\hline 183 & & organization_n \\
\hline 184 & & update_v \\
\hline 185 & * & basic_adj \\
\hline 186 & $*$ & specification_n \\
\hline 187 & & max_n \\
\hline 188 & * & configuration_n \\
\hline 189 & $\#$ & assumption_n \\
\hline 190 & & evaluation_n \\
\hline 191 & & processing_n \\
\hline 192 & & notation_n \\
\hline 193 & $*$ & storage_n \\
\hline 194 & * & protocol $n$ \\
\hline
\end{tabular}

\begin{tabular}{|c|c|c|}
\hline 196 & & typically_adv \\
\hline 197 & $*$ & subset_n \\
\hline 198 & $*$ & bound_n \\
\hline 199 & & respectively_adv \\
\hline 200 & $*$ & traffic_n \\
\hline 201 & $*$ & recognize_v \\
\hline 202 & $*$ & depth_n \\
\hline 203 & $*$ & attribute_n \\
\hline 204 & $*$ & aspect_n \\
\hline 205 & $*$ & characteristic_n \\
\hline 206 & $*$ & combination_n \\
\hline 207 & $*$ & branch_n \\
\hline 208 & $*$ & card_n \\
\hline 209 & $*$ & category_n \\
\hline 210 & $*$ & conditional_adj \\
\hline 211 & 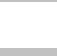 & modify_v \\
\hline 212 & $*$ & frequency_n \\
\hline 213 & $*$ & grid_n \\
\hline 214 & $*$ & global_adj \\
\hline 215 & $*$ & dimension_n \\
\hline 216 & $*$ & insert_v \\
\hline 217 & $*$ & priority_n \\
\hline 218 & $*$ & speed_n \\
\hline 219 & $*$ & variable_adj \\
\hline 220 & & center_n \\
\hline 221 & & exploit_v \\
\hline 222 & $*$ & link_v \\
\hline 223 & $*$ & measure_n \\
\hline 224 & $*$ & library_n \\
\hline 225 & 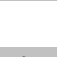 & similarity_n \\
\hline 226 & $*$ & iteration_n \\
\hline 227 & $*$ & vertex_n \\
\hline 228 & $*$ & resolution_n \\
\hline 229 & & prediction_n \\
\hline 230 & $*$ & transaction_n \\
\hline 231 & $\#$ & equivalent_adj \\
\hline 232 & & extract_v \\
\hline 233 & $\#$ & finite_adj \\
\hline 234 & $*$ & cache_n \\
\hline 235 & $\#$ & efficiency_n \\
\hline 236 & $*$ & label_n \\
\hline 237 & & valid_adj \\
\hline 238 & & functionality_n \\
\hline
\end{tabular}




\begin{tabular}{|c|c|c|}
\hline 239 & & summary_n \\
\hline 240 & $*$ & check_n \\
\hline 241 & & boundary_n \\
\hline 242 & & computing_n \\
\hline 243 & \# & multiplication_n \\
\hline 244 & & yield_v \\
\hline 245 & & automatically_adv \\
\hline 246 & & correspond_v \\
\hline 247 & & neighbor_n \\
\hline 248 & $\#$ & minimum_adj \\
\hline 249 & $*$ & compiler_- $\mathrm{n}$ \\
\hline 250 & & mathematical_adj \\
\hline 251 & & assignment_n \\
\hline 252 & & integrate_v \\
\hline 253 & \# & convert_v \\
\hline 254 & $*$ & order_v \\
\hline 255 & & related_adj \\
\hline 256 & $*$ & base_n \\
\hline 257 & & encode_v \\
\hline 258 & $*$ & segment_n \\
\hline 259 & & computational_adj \\
\hline 260 & & dimensional_adj \\
\hline 261 & $*$ & metric_n \\
\hline 262 & $*$ & fast_adj \\
\hline 263 & \# & zero_adj \\
\hline 264 & $*$ & label_v \\
\hline 265 & $*$ & list_v \\
\hline 266 & $*$ & safety_n \\
\hline 267 & & embed_v \\
\hline 268 & & allocate_v \\
\hline 269 & $*$ & minimize_v $v$ \\
\hline 270 & & desire_v \\
\hline 271 & $*$ & copy_n \\
\hline 272 & & classify_v \\
\hline 273 & $*$ & track_v \\
\hline 274 & & overview_n \\
\hline 275 & \# & diagram_ñ \\
\hline 276 & $*$ & active_adj \\
\hline 277 & $*$ & monitor_v \\
\hline 278 & $*$ & delete_v \\
\hline 279 & & temporal_adj \\
\hline 280 & & malicious_adj \\
\hline 281 & $*$ & drive_n \\
\hline 282 & & summarize_v \\
\hline
\end{tabular}

\begin{tabular}{|c|c|}
\hline 283 & * logical_adj \\
\hline 284 & * map_v \\
\hline 285 & * volume_n \\
\hline 286 & usage_n \\
\hline 287 & * min_n \\
\hline 288 & \# approximation_n \\
\hline 289 & * chain_n \\
\hline 290 & * name_v \\
\hline 291 & * bind_v \\
\hline 292 & \# count_n \\
\hline 293 & guarantee_v \\
\hline 294 & * map_n \\
\hline 295 & * format_n \\
\hline 296 & * channel_n \\
\hline 297 & update_n \\
\hline 298 & infrastructure_n \\
\hline 299 & adapt_v \\
\hline 300 & * stream_n \\
\hline 301 & * search_v \\
\hline 302 & * store_n \\
\hline 303 & capability_n \\
\hline 304 & * buffer_n \\
\hline 305 & \# ratio_n \\
\hline 306 & * engine_n \\
\hline 307 & * mapping_n \\
\hline 308 & optimize_v \\
\hline 309 & * kernel_n \\
\hline 310 & overhead_n \\
\hline 311 & * transformation_n \\
\hline 312 & recall_n \\
\hline 313 & print_v \\
\hline 314 & * feedback_n \\
\hline 315 & hypothesis_n \\
\hline 316 & * mean_adj \\
\hline 317 & * command_n \\
\hline 318 & * bound_v \\
\hline 319 & translate_v \\
\hline 320 & * translation_n \\
\hline 321 & verify_v \\
\hline 322 & arbitrary_adj \\
\hline 323 & limitation_n \\
\hline 324 & deploy_v \\
\hline 325 & spatial_adj \\
\hline 326 & threshold_n \\
\hline
\end{tabular}




\begin{tabular}{|c|c|}
\hline 327 & * inference_n \\
\hline 328 & $\#$ coefficient_n \\
\hline 329 & * view_v \\
\hline 330 & * video_adj \\
\hline 331 & * core $\mathrm{n}$ \\
\hline 332 & destination_n \\
\hline 333 & underlie_v \\
\hline 334 & * abstraction $\mathrm{n}$ \\
\hline 335 & * switch_v \\
\hline 336 & \# regression_n \\
\hline 337 & * learning_n \\
\hline 338 & square_n \\
\hline 339 & * entity_n \\
\hline 340 & * code_v \\
\hline 341 & * precision_n \\
\hline 342 & * extension_n \\
\hline 343 & * verification_n \\
\hline 344 & * interactive_adj \\
\hline 345 & * capacity_n \\
\hline 346 & * variation_n \\
\hline 347 & * file_v \\
\hline 348 & deployment_n \\
\hline 349 & specified_adj \\
\hline 350 & abstract_adj \\
\hline 351 & decrease_v \\
\hline 352 & \# one_n \\
\hline 353 & continuous_adj \\
\hline 354 & utilize_v \\
\hline 355 & * noise_n \\
\hline 356 & \# discrete_adj \\
\hline 357 & $\#$ curve_n \\
\hline 358 & rotation_n \\
\hline 359 & correctly_adv \\
\hline 360 & oriented_adj \\
\hline 361 & randomly_adv \\
\hline 362 & * hierarchy_n \\
\hline 363 & monitoring_n \\
\hline 364 & * fraction_n \\
\hline 365 & * default_n \\
\hline 366 & explicitly_adv \\
\hline 367 & * clock_n \\
\hline 368 & technical_adj \\
\hline 369 & \# simplify_v \\
\hline 370 & cross_n \\
\hline
\end{tabular}

\begin{tabular}{|c|c|c|}
\hline 371 & & simulate_v \\
\hline 372 & & characterize_v \\
\hline 373 & $*$ & switch_n \\
\hline 374 & $\#$ & scale_v \\
\hline 375 & & namely_adv \\
\hline 376 & & generalize_v \\
\hline 377 & & organize_v \\
\hline 378 & 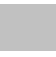 & interact_v \\
\hline 379 & $*$ & sequential_adj \\
\hline 380 & & allocation_n \\
\hline 381 & $*$ & developer_n \\
\hline 382 & & self_n \\
\hline 383 & & transfer_n \\
\hline 384 & $\#$ & rectangle_n \\
\hline 385 & $*$ & shift_n \\
\hline 386 & $\#$ & maximum_n \\
\hline 387 & $*$ & partition_v \\
\hline 388 & $*$ & prototype_n \\
\hline 389 & & explicit_adj \\
\hline 390 & 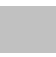 & slot_n \\
\hline 391 & $*$ & generic_adj \\
\hline 392 & $\#$ & estimation_n \\
\hline 393 & & sparse_adj \\
\hline 394 & & calculation_n \\
\hline 395 & $*$ & partition_n \\
\hline 396 & & commonly_adv \\
\hline 397 & & portion_n \\
\hline 398 & $*$ & chip_n \\
\hline 399 & & hierarchical_adj \\
\hline 400 & $*$ & signature_n \\
\hline 401 & $\#$ & remainder_n \\
\hline 402 & & graphical_adj \\
\hline 403 & & compile_v \\
\hline 404 & $\#$ & exact_adj \\
\hline 405 & & evolution_n \\
\hline 406 & $*$ & construct_n \\
\hline 407 & $*$ & scope_n \\
\hline 408 & $*$ & review_v \\
\hline 409 & $*$ & availability_n \\
\hline 410 & $\#$ & arithmetic_n \\
\hline 411 & $*$ & block_v \\
\hline 412 & & partial_adj \\
\hline 413 & & interpret_v \\
\hline 414 & & transfer_v \\
\hline
\end{tabular}




\begin{tabular}{|c|c|c|}
\hline 415 & $*$ & topology_n \\
\hline 416 & $\#$ & sample_v \\
\hline 417 & & taxonomy_n \\
\hline 418 & & second adv \\
\hline 419 & & terminate_v \\
\hline 420 & $*$ & counter_n \\
\hline 421 & $*$ & read_n \\
\hline 422 & * & split_v \\
\hline 423 & $*$ & increment_n \\
\hline 424 & $*$ & decomposition_n \\
\hline 425 & & left_n \\
\hline 426 & $*$ & register_v \\
\hline 427 & $\#$ & axis_n \\
\hline 428 & $*$ & syntax_n \\
\hline 429 & & frequent_adj \\
\hline 430 & $*$ & parallel_adj \\
\hline 431 & $*$ & influence_v \\
\hline 432 & & peer_n \\
\hline 433 & $*$ & null_adj \\
\hline 434 & & greedy_adj \\
\hline 435 & & efficiently_adv \\
\hline 436 & & evolve_v \\
\hline 437 & $\#$ & minimum_n \\
\hline 438 & & geometric_adj \\
\hline 439 & & dependency_n \\
\hline 440 & & engineer_n \\
\hline 441 & & distinguish_v \\
\hline 442 & $*$ & filter_v \\
\hline 443 & & equal_v \\
\hline 444 & & target_v \\
\hline 445 & & following_n \\
\hline 446 & $*$ & validation_n \\
\hline 447 & & variant_n \\
\hline 448 & $*$ & digital_adj \\
\hline 449 & & toward_con \\
\hline 450 & $*$ & width_n \\
\hline 451 & $\#$ & velocity_n \\
\hline 452 & & probabilistic_adj \\
\hline 453 & $*$ & pass_n \\
\hline 454 & & communicate_v \\
\hline 455 & & exceed_v \\
\hline 456 & & straightforward_adj \\
\hline 457 & $*$ & edit_v \\
\hline 458 & & automatic_adj \\
\hline
\end{tabular}

\begin{tabular}{|c|c|c|}
\hline 459 & & universal_adj \\
\hline 460 & i & in_adv \\
\hline 461 & \# & random_n \\
\hline 462 & $*$ & header_n \\
\hline 463 & & constrain_v \\
\hline 464 & $*$ & identifier_n \\
\hline 465 & $\#$ & compute_n \\
\hline 466 & $*$ & mouse_n \\
\hline 467 & $*$ & range_v \\
\hline 468 & $*$ & float_v \\
\hline 469 & & eliminate_v \\
\hline 470 & 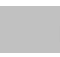 & multiply_v̄ \\
\hline 471 & $*$ & browser_n \\
\hline 472 & & violate_v \\
\hline 473 & & runtime_n \\
\hline 474 & 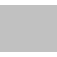 & hint_n \\
\hline 475 & $*$ & integrity_n \\
\hline 476 & $*$ & filter_n \\
\hline 477 & $\#$ & infinite_adj \\
\hline 478 & $*$ & likelihood_n \\
\hline 479 & $\#$ & multiple_n \\
\hline 480 & $\#$ & zero_n \\
\hline 481 & & theoretical_adj \\
\hline 482 & & occurrence_n \\
\hline 483 & 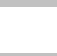 & infer_v \\
\hline 484 & $\#$ & initialize_v \\
\hline 485 & & completion_n \\
\hline 486 & $*$ & constant_n \\
\hline 487 & & adaptive_adj \\
\hline 488 & $*$ & maintenance_n \\
\hline 489 & & aggregation_n \\
\hline 490 & & compromise_v \\
\hline 491 & & symmetric_adj \\
\hline 492 & & artifact_n \\
\hline 493 & $*$ & interrupt_v \\
\hline 494 & & reuse_v \\
\hline 495 & & bin_n \\
\hline 496 & $*$ & paradigm_n \\
\hline 497 & $*$ & type_v \\
\hline 498 & & discovery_n \\
\hline 499 & $*$ & identification_n \\
\hline 500 & & coordinate_n \\
\hline 501 & & vendor_n \\
\hline 502 & $*$ & expectation_n \\
\hline
\end{tabular}




\begin{tabular}{|c|c|c|}
\hline 503 & & retrieval_n \\
\hline 504 & & independently_adv \\
\hline 505 & $*$ & maximize_v \\
\hline 506 & $\#$ & pi_n \\
\hline 507 & & reasoning_n \\
\hline 508 & $*$ & inequality_n \\
\hline 509 & & schedule_v \\
\hline 510 & & transmission_n \\
\hline 511 & & modification_n \\
\hline 512 & & coarse_adj \\
\hline 513 & $*$ & highlight_v \\
\hline 514 & & statistical_adj \\
\hline 515 & $\#$ & approximate_v \\
\hline 516 & $*$ & overlap_v - \\
\hline 517 & $*$ & shift_v \\
\hline 518 & $\#$ & robust_adj \\
\hline 519 & & trace_v \\
\hline 520 & $*$ & scan_v \\
\hline 521 & $*$ & refinement_n \\
\hline 522 & & yield_n \\
\hline 523 & & transmit_v \\
\hline 524 & $*$ & connectivity_n \\
\hline 525 & & recursively_adv \\
\hline 526 & $*$ & function_v \\
\hline 527 & & faster_adv \\
\hline 528 & $*$ & outlier_n \\
\hline 529 & & preceding_adj \\
\hline 530 & $*$ & bug_n \\
\hline 531 & $*$ & recovery_n \\
\hline 532 & $*$ & overflow_n \\
\hline 533 & $*$ & gate_n \\
\hline 534 & $\#$ & empirical_adj \\
\hline 535 & & intermediate_adj \\
\hline 536 & & effectiveness_n \\
\hline 537 & & guideline_n \\
\hline 538 & $\#$ & collision_n \\
\hline 539 & & comparable_adj \\
\hline 540 & & formulation_n \\
\hline 541 & & exhibit_v \\
\hline 542 & & roughly_adv \\
\hline 543 & & operational_adj \\
\hline 544 & & minimal_adj \\
\hline 545 & & automated_adj \\
\hline 546 & $*$ & split_n \\
\hline
\end{tabular}

\begin{tabular}{|c|c|c|}
\hline 547 & & retrieve_v \\
\hline 548 & & division_n \\
\hline 549 & & content_adj \\
\hline 550 & $*$ & deterministic_adj \\
\hline 551 & $*$ & port_n \\
\hline 552 & $\#$ & prime_n \\
\hline 553 & & simultaneously_adv \\
\hline 554 & $*$ & intersection_n \\
\hline 555 & $*$ & scalability_n \\
\hline 556 & $\#$ & utility_n \\
\hline 557 & $\#$ & uniform_adj \\
\hline 558 & $*$ & object_v \\
\hline 559 & $*$ & electronic_adj \\
\hline 560 & & structural_adj \\
\hline 561 & & nonnegative_adj \\
\hline 562 & $\#$ & implicit_adj \\
\hline 563 & & arrow_n \\
\hline 564 & & insight_n \\
\hline 565 & & survey_v \\
\hline 566 & & prior_adj \\
\hline 567 & & experimental_adj \\
\hline 568 & $*$ & composition_n \\
\hline 569 & & weighted_adj \\
\hline 570 & & hybrid_adj \\
\hline 571 & & adjacent_adj \\
\hline 572 & & alignment_n \\
\hline 573 & $*$ & install_v \\
\hline 574 & & manipulate_v \\
\hline 575 & & incorrect_adj \\
\hline 576 & & motivate_v \\
\hline 577 & & dynamically_adv \\
\hline 578 & & depict_v \\
\hline 579 & & enforce_v \\
\hline 580 & & coordinate_v \\
\hline 581 & $*$ & null_n \\
\hline 582 & & zero_v \\
\hline 583 & $*$ & alphabet_n \\
\hline 584 & & vulnerable_adj \\
\hline 585 & $*$ & rank_v \\
\hline 586 & & utilization_n \\
\hline 587 & & layout_n \\
\hline 588 & & precise_adj \\
\hline 589 & $\#$ & dynamics_n \\
\hline 590 & $*$ & sampling_n \\
\hline
\end{tabular}




\begin{tabular}{|c|c|c|}
\hline 591 & & offset_v \\
\hline 592 & & convention_n \\
\hline 593 & $*$ & bias_n \\
\hline 594 & & simplicity_n \\
\hline 595 & & proportional_adj \\
\hline 596 & $*$ & interpretation_n \\
\hline 597 & & tracking_n \\
\hline 598 & $*$ & validate_v \\
\hline 599 & & flip_v \\
\hline 600 & $*$ & graphics_n \\
\hline 601 & \# & mechanical_adj \\
\hline 602 & & mine_v \\
\hline 603 & & phenomenon_n \\
\hline 604 & $*$ & matching_n \\
\hline 605 & $*$ & equivalence_n \\
\hline 606 & & principal_adj \\
\hline 607 & & double_adv \\
\hline 608 & & legitimate_adj \\
\hline 609 & $*$ & monitor_n \\
\hline 610 & & enterprise_n \\
\hline 611 & & prior_adv \\
\hline 612 & $*$ & generator_n \\
\hline 613 & & smart_adj \\
\hline 614 & \# & correction_n \\
\hline 615 & & checking_n \\
\hline 616 & $*$ & completeness_n \\
\hline 617 & $*$ & projection_n \\
\hline 618 & $*$ & document_v \\
\hline 619 & & rewrite_v \\
\hline 620 & & helpful_adj \\
\hline 621 & & flexible_adj \\
\hline 622 & & request_v \\
\hline 623 & & shared_adj \\
\hline 624 & & correctness_n \\
\hline 625 & \# & trace_n \\
\hline 626 & & additionally_adv \\
\hline 627 & \# & load_v \\
\hline 628 & & connected_adj \\
\hline 629 & & behave_v \\
\hline 630 & & host_v \\
\hline 631 & & numérical_adj \\
\hline 632 & $\#$ & arithmetic_adj \\
\hline 633 & & workload_n \\
\hline 634 & & sharing_ $\mathrm{n}$ \\
\hline
\end{tabular}

\begin{tabular}{|c|c|c|}
\hline 635 & & guarantee_n \\
\hline 636 & $*$ & indicator_n \\
\hline 637 & $*$ & remote_adj \\
\hline 638 & & equality_n \\
\hline 639 & & centralized_adj \\
\hline 640 & $*$ & chunk_n \\
\hline 641 & & originally_adv \\
\hline 642 & & formally_adv \\
\hline 643 & 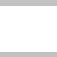 & repeatedly_adv \\
\hline 644 & $*$ & metric_adj \\
\hline 645 & & intuitive_adj \\
\hline 646 & & configure_v \\
\hline 647 & & span_v \\
\hline 648 & $*$ & inverse_n \\
\hline 649 & & regardless_adv \\
\hline 650 & & binary_n \\
\hline 651 & & incremental_adj \\
\hline 652 & & double_v \\
\hline 653 & $*$ & primitive_adj \\
\hline 654 & & normalize_v \\
\hline 655 & & versus_con \\
\hline 656 & & anonymous_adj \\
\hline 657 & $*$ & trigger_v \\
\hline 658 & & accomplish_v \\
\hline 659 & $*$ & structure_v \\
\hline 660 & $*$ & repository_n \\
\hline 661 & & replacement_n \\
\hline 662 & & penalty_n \\
\hline 663 & & tolerance_n \\
\hline 664 & & metadata_n \\
\hline 665 & & structured_adj \\
\hline 666 & & reference_v \\
\hline 667 & & complicated_adj \\
\hline 668 & $*$ & group_v \\
\hline 669 & & exponential_adj \\
\hline 670 & & swap_n \\
\hline 671 & $*$ & signal_v \\
\hline 672 & & omit_v \\
\hline 673 & & on_adv \\
\hline 674 & $\#$ & even_adj \\
\hline 675 & & exchange_v \\
\hline 676 & & executable_adj \\
\hline 677 & & extraction_n \\
\hline 678 & & schedule_n \\
\hline
\end{tabular}




\begin{tabular}{|c|c|c|}
\hline 679 & & handling_n \\
\hline 680 & $*$ & website_n \\
\hline 681 & $*$ & root_v \\
\hline 682 & & accessible adj \\
\hline 683 & & propagate_v \\
\hline 684 & \# & randomize_v \\
\hline 685 & $*$ & permutation_n \\
\hline 686 & & advanced_adj \\
\hline 687 & & integrated_adj \\
\hline 688 & $*$ & arc_n \\
\hline 689 & & latter_adj \\
\hline 690 & & align_v \\
\hline 691 & $\#$ & approximate_adj \\
\hline 692 & & vertical_adj \\
\hline 693 & $*$ & stream_v \\
\hline 694 & & besides_con \\
\hline 695 & & rotate_v \\
\hline 696 & $*$ & core_adj \\
\hline 697 & $*$ & scan_n \\
\hline 698 & & nest_v \\
\hline 699 & & journal_n \\
\hline 700 & & subject_adj \\
\hline 701 & & briefly_adv \\
\hline 702 & $*$ & base_adj \\
\hline 703 & & diversity_n \\
\hline 704 & \# & fit_n \\
\hline 705 & \# & concurrent_adj \\
\hline 706 & $*$ & annotation_n \\
\hline 707 & & appendix_n \\
\hline 708 & \# & implication_n \\
\hline 709 & & sufficiently_adv \\
\hline 710 & $*$ & consistency_n \\
\hline 711 & & weakness_n \\
\hline 712 & & motivation_n \\
\hline 713 & & formulate_v \\
\hline 714 & & center_v \\
\hline 715 & & periodic_adj \\
\hline 716 & & adoption_n \\
\hline 717 & $*$ & pop_v \\
\hline 718 & & compatible_adj \\
\hline 719 & & decrease_n \\
\hline 720 & $\#$ & correspondence_n \\
\hline 721 & & swap_v \\
\hline 722 & & receiver_n \\
\hline
\end{tabular}

\begin{tabular}{|c|c|c|}
\hline 723 & & reconstruction_n \\
\hline 724 & $\#$ & quantity_n \\
\hline 725 & & distinction_n \\
\hline 726 & $*$ & scalable_adj \\
\hline 727 & & alternatively_adv \\
\hline 728 & & stable_adj \\
\hline 729 & $*$ & disjoint_adj \\
\hline 730 & & convenient_adj \\
\hline 731 & & consecutive_adj \\
\hline 732 & & query_v \\
\hline 733 & $\#$ & displacement_n \\
\hline 734 & & adaptation_n \\
\hline 735 & & project_v \\
\hline 736 & & distortion_n \\
\hline 737 & & extensive_adj \\
\hline 738 & & placement_n \\
\hline 739 & & separation_n \\
\hline 740 & $\#$ & feasible_adj \\
\hline 741 & & networking_n \\
\hline 742 & $*$ & tail_n \\
\hline 743 & & violation_n \\
\hline 744 & & annotate_v \\
\hline 745 & & offset_n \\
\hline 746 & $*$ & conjunction_n \\
\hline 747 & & generalization_n \\
\hline 748 & $\#$ & exclusive_adj \\
\hline 749 & & categorize_v \\
\hline 750 & $*$ & dictionary_n \\
\hline 751 & & debug_v \\
\hline 752 & & emphasize_v \\
\hline 753 & & applicable_adj \\
\hline 754 & & bottom_adj \\
\hline 755 & & uniformly_adv \\
\hline 756 & & conversion_n \\
\hline 757 & & magnitude_n \\
\hline 758 & & modified_adj \\
\hline 759 & & iterative_adj \\
\hline 760 & $*$ & template_n \\
\hline 761 & & thereby_adv \\
\hline 762 & $*$ & parallel_n \\
\hline 763 & $*$ & heuristic_adj \\
\hline 764 & $\#$ & calculus_n \\
\hline 765 & $*$ & program_v \\
\hline 766 & & separately_adv \\
\hline
\end{tabular}




\begin{tabular}{|c|c|c|}
\hline 767 & $*$ & balance_v \\
\hline 768 & * & controller_n \\
\hline 769 & & greatly_adv \\
\hline 770 & * & attribute $v$ \\
\hline 771 & & acquisition_n \\
\hline 772 & & purchase_v \\
\hline 773 & & flexibility_n \\
\hline 774 & & quantify_v \\
\hline 775 & & logically_adv \\
\hline 776 & $*$ & robustness_n \\
\hline 777 & $*$ & assertion_ $\mathrm{n}$ \\
\hline 778 & & derivation_n \\
\hline 779 & & manually_adv \\
\hline 780 & & reside_v \\
\hline 781 & $*$ & draw_n \\
\hline 782 & & numerous_adj \\
\hline 783 & & manual_adj \\
\hline 784 & & reverse_adj \\
\hline 785 & $*$ & uncertainty_n \\
\hline 786 & & desirable_adj \\
\hline 787 & $*$ & speed_v \\
\hline 788 & & timing_n \\
\hline 789 & $*$ & click_n \\
\hline 790 & & million_n \\
\hline 791 & & realistic_adj \\
\hline 792 & & peer_v \\
\hline 793 & & augment_v \\
\hline 794 & & anomaly_n \\
\hline 795 & & nonzero_adj \\
\hline 796 & & self_adj \\
\hline 797 & & trivial_adj \\
\hline 798 & & specialized_adj \\
\hline 799 & & discard_v \\
\hline 800 & & observed_adj \\
\hline 801 & & accurately_adv \\
\hline 802 & & validity_n \\
\hline 803 & & strictly_adv \\
\hline 804 & & mitigate_v \\
\hline 805 & $*$ & push_n \\
\hline 806 & $\#$ & square_v \\
\hline 807 & $*$ & planning_n \\
\hline 808 & $\#$ & above_adv \\
\hline 809 & & persistent_adj \\
\hline 810 & & partially_adv \\
\hline
\end{tabular}

\begin{tabular}{|c|c|c|}
\hline 811 & & traverse_v \\
\hline 812 & $*$ & balanced_adj \\
\hline 813 & & refine_v \\
\hline 814 & & required_adj \\
\hline 815 & $*$ & click_v \\
\hline 816 & & meaningful_adj \\
\hline 817 & & incur_v \\
\hline 818 & & precede_v \\
\hline 819 & & collaborative_adj \\
\hline 820 & & heterogeneous_adj \\
\hline 821 & & marketing_n \\
\hline 822 & & terminology_n \\
\hline 823 & & automate_v \\
\hline 824 & & define_v \\
\hline 825 & & flash_n \\
\hline 826 & $*$ & algorithm, 829) \\
\hline 827 & & continuously_adv \\
\hline 828 & & number_v \\
\hline 829 & $*$ & forth_adv \\
\hline 830 & & subtract_v \\
\hline 831 & & orient_v \\
\hline 832 & & subsystem_n \\
\hline 833 & & disadvantage_n \\
\hline 834 & & replicate_v \\
\hline 835 & $*$ & closed_adj \\
\hline 836 & & implicitly_adv \\
\hline 837 & $\#$ & redundant_adj \\
\hline 838 & & mutual_adj \\
\hline 839 & & parallelism_n \\
\hline 840 & & alter_v \\
\hline 841 & & selected_adj \\
\hline 842 & & manipulation_n \\
\hline 843 & $*$ & simulator_n \\
\hline 844 & $*$ & bucket_n \\
\hline 845 & & horizontal_adj \\
\hline 846 & & mask_n \\
\hline 847 & & collaboration_n \\
\hline 848 & $*$ & printer_n \\
\hline 849 & & challenging_adj \\
\hline 850 & & classical_adj \\
\hline 851 & & due_adv \\
\hline 852 & & exit_n \\
\hline 853 & $*$ & routine_adj \\
\hline 854 & $*$ & multimedia_n \\
\hline
\end{tabular}




\begin{tabular}{|c|c|c|}
\hline 855 & & cope_v \\
\hline 856 & & illustration_n \\
\hline 857 & & incoming_adj \\
\hline 858 & $\#$ & representative_adj \\
\hline 859 & & inherent_adj \\
\hline 860 & $\#$ & sum_v \\
\hline 861 & & abstract_n \\
\hline 862 & & fragment_n \\
\hline 863 & $\#$ & composite_adj \\
\hline 864 & & relational_adj \\
\hline 865 & & assert_v \\
\hline 866 & & opposite_adj \\
\hline 867 & & vice_adj \\
\hline 868 & & neighboring_adj \\
\hline 869 & & snapshot_n \\
\hline 870 & & latter_n \\
\hline 871 & & accordingly_adv \\
\hline 872 & $\#$ & invalid_adj \\
\hline 873 & $*$ & visualization_n \\
\hline 874 & & size_v \\
\hline 875 & & novel_adj \\
\hline 876 & & physically_adv \\
\hline 877 & & locality_n \\
\hline 878 & & transform_n \\
\hline 879 & & concrete_adj \\
\hline
\end{tabular}

$\begin{array}{lll}880 & \text { computationally_adv } \\ 881 & \text { hidden_adj } \\ 882 & \text { fulfill_v } \\ 883 & \text { originate_v } \\ 884 & \text { virtual_n } \\ 885 & * & \text { script_n } \\ 886 & \text { acceptance_n } \\ 887 & \text { arbitrarily_adv } \\ 888 & \text { setup_n } \\ 889 & \text { \# } & \text { positive_n } \\ 890 & * & \text { shell_n } \\ 891 & \text { drawback_n } \\ 892 & * \text { scaling_n } \\ 893 & \text { \# } & \text { couple_v } \\ 894 & \text { brute_adj } \\ 895 & \text { average_v } \\ 896 & * & \text { benchmark_n } \\ 897 & \text { false_adj } \\ 898 & \text { designate_v } \\ 899 & \text { outer_adj } \\ 900 & * \text { derivative_n } \\ 901 & \text { classic_adj } \\ 902 & * \text { stack_v } \\ 903 & \text { next_adv } \\ 904 & \text { leverage_v } \\ & \\ \end{array}$




\section{Appendix B: The Computer Science Academic Vocabulary Supplemental}

\section{List (CSAVL-S)}

\section{Key:}

$\mathrm{n}$ : noun, v: verb, adj: adjective, adv: adverb

*: Computer science-specific meaning (listed in The Oxford Dictionary of Computer Science $7^{\text {th }}$ ed.) \#: Mathematics-specific meaning (listed in The Concise Oxford Dictionary of Mathematics, $4^{\text {th }}$ ed.) (items found in both dictionaries are only listed with asterisks)

Format:

(rank) (special meaning) (lemma)

\begin{tabular}{|c|c|c|}
\hline 1 & $*$ & string_n \\
\hline 2 & $*$ & integer_n \\
\hline 3 & $*$ & instruction_n \\
\hline 4 & $*$ & statement_n \\
\hline 5 & $*$ & sensor_n \\
\hline 6 & $*$ & disk_n \\
\hline 7 & $*$ & register_n \\
\hline 8 & \# & theorem_n \\
\hline 9 & $*$ & byte_n \\
\hline 10 & & thread_n \\
\hline 11 & $*$ & heap_n \\
\hline 12 & $*$ & accept_v \\
\hline 13 & \# & trajectory_n \\
\hline 14 & $*$ & privacy_n \\
\hline 15 & $*$ & video_n \\
\hline 16 & & attacker_n \\
\hline 17 & $*$ & pointer_n \\
\hline 18 & $*$ & mesh_n \\
\hline 19 & $*$ & password_n \\
\hline 20 & & hash_n \\
\hline 21 & $*$ & display_n \\
\hline 22 & & void_n \\
\hline 23 & $*$ & programmer_n \\
\hline 24 & $*$ & vulnerability_n \\
\hline 25 & $*$ & fault_n \\
\hline 26 & & provider_n \\
\hline
\end{tabular}

\begin{tabular}{|c|c|c|}
\hline 28 & & insertion_n \\
\hline 29 & $*$ & queue_n \\
\hline 30 & $*$ & lock_n \\
\hline 31 & 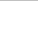 & merge_v \\
\hline 32 & $*$ & encryption_n \\
\hline 33 & $*$ & malware_n \\
\hline 34 & $*$ & radius_n \\
\hline 35 & $*$ & correlation_n \\
\hline 36 & $*$ & height_n \\
\hline 37 & & pipeline_n \\
\hline 38 & & classifier_n \\
\hline 39 & & invoke_v \\
\hline 40 & $*$ & circuit_n \\
\hline 41 & 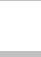 & assembly_n \\
\hline 42 & $*$ & directory_n \\
\hline 43 & \# & lemma_n \\
\hline 44 & $*$ & packet_n \\
\hline 45 & $*$ & recursive_adj \\
\hline 46 & $*$ & listing_n \\
\hline 47 & $*$ & methodology_n \\
\hline 48 & & virtualization_n \\
\hline 49 & $*$ & polynomial_adj \\
\hline 50 & \# & ray_n \\
\hline 51 & $*$ & overlay_n \\
\hline 52 & $*$ & bandwidth_n \\
\hline
\end{tabular}




\begin{tabular}{llll}
53 & $*$ & botnet_n \\
\hline 54 & $*$ & expansion_n \\
55 & $*$ & compression_n \\
56 & $\#$ & proposition_n \\
57 & & scheduling_n \\
58 & $*$ & insert_n \\
59 & & semantic_adj \\
60 & $*$ & tape_n \\
61 & & vertices_n \\
62 & $*$ & variance_n \\
63 & $*$ & reliability_n \\
64 & $*$ & unsigned_adj \\
65 & & censorship_n \\
66 & $*$ & density_n \\
67 & & modeling_n \\
68 & $*$ & latency_n \\
69 & & import_n \\
70 & & migration_n \\
71 & $\#$ & tensor_n \\
72 & & subproblem_n \\
73 & & mining_n \\
74 & $\#$ & deviation_n \\
75 & & pricing_n \\
76 & $\#$ & geometry_n \\
77 & $*$ & scanner_n \\
78 & & pane_n \\
79 & $*$ & subtree_n \\
80 & $*$ & sense_v \\
81 & & warp_n \\
82 & $*$ & routine_n \\
83 & $*$ & recurrence_n \\
84 & & usability_n \\
85 & $*$ & rank_n \\
86 & $*$ & induction_n \\
87 & & coin_n \\
88 & & encrypt_v \\
89 & $*$ & mod_n \\
90 & & clause_n \\
\hline 92 & & math_n \\
\hline & & constructor_n \\
\hline 6
\end{tabular}

\begin{tabular}{|c|c|c|}
\hline 93 & $*$ & complement_n \\
\hline 94 & $*$ & pixel_n \\
\hline 95 & $\#$ & particle_n \\
\hline 96 & & hash_v \\
\hline 97 & $\#$ & mathematics_n \\
\hline 98 & $\#$ & triangle_n \\
\hline 99 & $*$ & email_n \\
\hline 100 & $*$ & patch_n \\
\hline 101 & & print_n \\
\hline 102 & $*$ & jump_n \\
\hline 103 & & wire_n \\
\hline 104 & & creator_n \\
\hline 105 & & floating_adj \\
\hline 106 & $*$ & recursion_n \\
\hline 107 & $*$ & prompt_v \\
\hline 108 & $*$ & gesture_n \\
\hline 109 & $*$ & quantifier_n \\
\hline 110 & & propagation_n \\
\hline 111 & $*$ & leaf_n \\
\hline 112 & $*$ & grammar_n \\
\hline 113 & & amortize_v \\
\hline 114 & $*$ & operand_n \\
\hline 115 & $*$ & animation_n \\
\hline 116 & $*$ & button_n \\
\hline 117 & $*$ & suffix_n \\
\hline 118 & $*$ & quicksort_n \\
\hline 119 & & defense_n \\
\hline 120 & $*$ & wireless_n \\
\hline 121 & & nil_n \\
\hline 122 & & handler_n \\
\hline 123 & $*$ & scheduler_n \\
\hline 124 & $*$ & ontology_n \\
\hline 125 & & reuse_n \\
\hline 126 & & tactile_adj \\
\hline 127 & & needle_n \\
\hline 128 & & boolean_adj \\
\hline 129 & & deformation_n \\
\hline 130 & & decimal_adj \\
\hline 131 & $*$ & hazard_n \\
\hline 132 & & governance_n \\
\hline
\end{tabular}




\begin{tabular}{|c|c|c|c|c|c|}
\hline 133 & & rational_adj & 173 & & exploit_n \\
\hline 134 & $\#$ & binomial_adj & 174 & $*$ & rate_v \\
\hline 135 & & segmentation_n & 175 & $\#$ & plot_v \\
\hline 136 & & mobility_n & 176 & $*$ & tip_n \\
\hline 137 & $\#$ & sink_n & 177 & $*$ & blog_n \\
\hline 138 & $*$ & flag_n & 178 & & ethical_adj \\
\hline 139 & & cyber_adj & 179 & $\#$ & decidable_adj \\
\hline 140 & & taint_n & 180 & $\#$ & strut_n \\
\hline 141 & & inject_v & 181 & & calendar_n \\
\hline 142 & $*$ & predicate_n & 182 & $\#$ & polygon_n \\
\hline 143 & & meter_n & 183 & & numeric_adj \\
\hline 144 & $*$ & polynomial_n & 184 & & transportation_n \\
\hline 145 & & orientation_n & 185 & & voltage_n \\
\hline 146 & & joint_n & 186 & $*$ & handle_n \\
\hline 147 & $*$ & injection_n & 187 & & solver_n \\
\hline 148 & & deletion_n & 188 & & subclass_n \\
\hline 149 & $*$ & prefix_n & 189 & $\#$ & pivot_n \\
\hline 150 & $*$ & hypervisor_n & 190 & $*$ & delete_n \\
\hline 151 & & decode_v & 191 & & judgment_n \\
\hline 152 & $*$ & routing_n & 192 & & enforcement_n \\
\hline 153 & & organizational_adj & 193 & & neighborhood_n \\
\hline 154 & & divide_n & 194 & $\#$ & rod_n \\
\hline 155 & $*$ & tuple_n & 195 & & correlate_v \\
\hline 156 & & modulo_n & 196 & $*$ & negation_n \\
\hline 157 & & gadget_n & 197 & $*$ & documentation_n \\
\hline 158 & $*$ & certificate_n & 198 & & rout_v \\
\hline 159 & & architectural_adj & 199 & $*$ & token_n \\
\hline 160 & & inductive_adj & 200 & $*$ & worm_n \\
\hline 161 & $*$ & semantics_n & 201 & & flip_n \\
\hline 162 & $*$ & contradiction_n & 202 & & successor_n \\
\hline 163 & $*$ & subsequence_n & 203 & & hexadecimal_n \\
\hline 164 & & subarray_n & 204 & $*$ & clone_n \\
\hline 165 & & orchestration_n & 205 & $*$ & convergence_n \\
\hline 166 & $\#$ & acceleration_n & 206 & & atomic_adj \\
\hline 167 & $*$ & histogram_n & 207 & & navigation_n \\
\hline 168 & $*$ & module_n & 208 & & lens_n \\
\hline 169 & & radar_n & 209 & & wait_n \\
\hline 170 & $*$ & authentication_n & 210 & & recommender_n \\
\hline 171 & & agile_adj & 211 & & pump_v \\
\hline 172 & $*$ & slice_n & 212 & & nondeterministic_adj \\
\hline
\end{tabular}




\begin{tabular}{|c|c|c|}
\hline 213 & & drift_n \\
\hline 214 & & maximal_adj \\
\hline 215 & & intrusion_n \\
\hline 216 & & stochastic_adj \\
\hline 217 & $*$ & interpolation_n \\
\hline 218 & & symmetry_n \\
\hline 219 & & novice_n \\
\hline 220 & $*$ & norm_n \\
\hline 221 & & join_n \\
\hline 222 & & fetch_v \\
\hline 223 & & interference_n \\
\hline 224 & & guess_n \\
\hline 225 & $\#$ & divisor_n \\
\hline 226 & & deadline_n \\
\hline 227 & $*$ & bot_n \\
\hline 228 & $*$ & spam_n \\
\hline 229 & $*$ & schema_n \\
\hline 230 & & latent_adj \\
\hline 231 & & propositional_adj \\
\hline 232 & & congruence_n \\
\hline 233 & $*$ & median_n \\
\hline 234 & $*$ & interoperability_n \\
\hline 235 & $*$ & localization_n \\
\hline 236 & $*$ & throughput_n \\
\hline 237 & & legacy_n \\
\hline 238 & & probe_v \\
\hline 239 & $*$ & declaration_n \\
\hline 240 & & stakeholder_n \\
\hline 241 & & superclass_n \\
\hline 242 & $*$ & automaton_n \\
\hline 243 & & fusion_n \\
\hline 244 & & cognitive_adj \\
\hline 245 & & printing_n \\
\hline 246 & & resilience_n \\
\hline 247 & & congestion_n \\
\hline 248 & & hologram_n \\
\hline 249 & & probe_n \\
\hline 250 & $*$ & literal_n \\
\hline 251 & $*$ & cipher_n \\
\hline 252 & & override_n \\
\hline
\end{tabular}

\begin{tabular}{|c|c|c|}
\hline 253 & $*$ & asymptotic_adj \\
\hline 254 & $*$ & login_n \\
\hline 255 & & interpolate_v \\
\hline 256 & & license_n \\
\hline 257 & & iterator_n \\
\hline 258 & $*$ & float_n \\
\hline 259 & $*$ & invariant_n \\
\hline 260 & $\#$ & aggregate_v \\
\hline 261 & & compound_adj \\
\hline 262 & & grain_v \\
\hline 263 & & quadratic_adj \\
\hline 264 & & indoor_adj \\
\hline 265 & & deformable_adj \\
\hline 266 & $*$ & pseudocode_n \\
\hline 267 & $*$ & find_n \\
\hline 268 & & factorization_n \\
\hline 269 & & intellectual_adj \\
\hline 270 & & relevance_n \\
\hline 271 & $*$ & phishing_n \\
\hline 272 & $*$ & loop_v \\
\hline 273 & $*$ & hacker_n \\
\hline 274 & & stage_v \\
\hline 275 & & patient_adj \\
\hline 276 & & spin_n \\
\hline 277 & $\#$ & gram_n \\
\hline 278 & $*$ & covariance_n \\
\hline 279 & & pose_n \\
\hline 280 & $\#$ & elastic_adj \\
\hline 281 & & automata_n \\
\hline 282 & & texture_n \\
\hline 283 & & bubble_n \\
\hline 284 & $\#$ & divisible_adj \\
\hline 285 & $\#$ & gradient_n \\
\hline 286 & $*$ & parity_n \\
\hline 287 & & deck_n \\
\hline 288 & $*$ & hop_n \\
\hline 289 & $*$ & trap_n \\
\hline 290 & $*$ & redundancy_n \\
\hline 291 & & adversary_n \\
\hline 292 & $*$ & multiprocessor_n \\
\hline
\end{tabular}




\begin{tabular}{|c|c|c|}
\hline 293 & & zoom_v \\
\hline 294 & $*$ & confidentiality_n \\
\hline 295 & & undirected_adj \\
\hline 296 & $*$ & decryption_n \\
\hline 297 & $*$ & perception_n \\
\hline 298 & & mathematician_n \\
\hline 299 & $*$ & dual_adj \\
\hline 300 & $*$ & virus_n \\
\hline 301 & & quantification_n \\
\hline 302 & & virtue_n \\
\hline 303 & & jammer_n \\
\hline 304 & . & rigid_adj \\
\hline 305 & & puzzle_n \\
\hline 306 & $*$ & descriptor_n \\
\hline 307 & $*$ & keyword_n \\
\hline 308 & 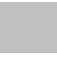 & tissue_n \\
\hline 309 & $*$ & branch_v \\
\hline 310 & & neutrality_n \\
\hline 311 & $*$ & synchronization_n \\
\hline 312 & & diagonal_adj \\
\hline 313 & & commerce_n \\
\hline 314 & $*$ & stability_n \\
\hline 315 & & conquer_v \\
\hline 316 & $*$ & patent_n \\
\hline 317 & & enter_n \\
\hline 318 & 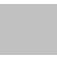 & optical_adj \\
\hline 319 & $*$ & concurrency_n \\
\hline 320 & & geolocation_n \\
\hline 321 & & calibration_n \\
\hline 322 & $*$ & overwrite_v \\
\hline 323 & & jamming_n \\
\hline 324 & & accelerometer_n \\
\hline 325 & & facial_adj \\
\hline 326 & $*$ & app_n \\
\hline 327 & $*$ & axiom_n \\
\hline 328 & & true_n \\
\hline 329 & & surveillance_n \\
\hline 330 & & confidential_adj \\
\hline 331 & & appliance_n \\
\hline 332 & $*$ & hex_n \\
\hline
\end{tabular}

\begin{tabular}{|c|c|c|}
\hline 333 & $*$ & proxy_n \\
\hline 334 & & textual_adj \\
\hline 335 & $*$ & proof_adj \\
\hline 336 & $*$ & ciphertext_n \\
\hline 337 & & reusable_adj \\
\hline 338 & & current_n \\
\hline 339 & & pipeline_v \\
\hline 340 & & catch_n \\
\hline 341 & & footer_n \\
\hline 342 & & mobile_n \\
\hline 343 & $*$ & granularity_n \\
\hline 344 & & summation_n \\
\hline 345 & $*$ & download_n \\
\hline 346 & & ecosystem_n \\
\hline 347 & & conjecture_n \\
\hline 348 & & multipath_n \\
\hline 349 & & spin_v \\
\hline 350 & & idle_adj \\
\hline 351 & $*$ & exponent_n \\
\hline 352 & & contingency_n \\
\hline 353 & & wrapper_n \\
\hline 354 & $\#$ & eigenvalue_n \\
\hline 355 & $*$ & plaintext_n \\
\hline 356 & $*$ & gateway_n \\
\hline 357 & $*$ & deadlock_n \\
\hline 358 & & defect_n \\
\hline 359 & & magnetic_adj \\
\hline 360 & & docker_n \\
\hline 361 & $\#$ & express_v \\
\hline 362 & $*$ & scalar_adj \\
\hline 363 & & unsafe_adj \\
\hline 364 & & duplicate_adj \\
\hline 365 & $*$ & amplitude_n \\
\hline 366 & & ethic_n \\
\hline 367 & & scatter_n \\
\hline 368 & & spanner_n \\
\hline 369 & $*$ & malware_adj \\
\hline 370 & $*$ & offline_adj \\
\hline 371 & $*$ & substitution_n \\
\hline 372 & $\#$ & smooth_v \\
\hline
\end{tabular}




\begin{tabular}{|c|c|c|c|c|c|}
\hline 373 & & behavioral_adj & 413 & & indexing_n \\
\hline 374 & $*$ & page_v & 414 & & contextual_adj \\
\hline 375 & & builder_n & 415 & $*$ & semaphore_n \\
\hline 376 & $*$ & undecidable_adj & 416 & & thinner_n \\
\hline 377 & & blob_n & 417 & & tetrahedra_n \\
\hline 378 & $*$ & guest_adj & 418 & & extended_adj \\
\hline 379 & & constitute_v & 419 & & intensive_adj \\
\hline 380 & & respective_adj & 420 & & manufacturing_n \\
\hline 381 & & systematic_adj & 421 & & induce_v \\
\hline 382 & & outperform_v & 422 & $\#$ & decompose_v \\
\hline 383 & $*$ & marker_n & 423 & & intuition_n \\
\hline 384 & & color_v & 424 & & outgoing_adj \\
\hline 385 & & missing_adj & 425 & & super_adj \\
\hline 386 & & thickness_n & 426 & & anonymity_n \\
\hline 387 & $\#$ & convex_adj & 427 & $\#$ & ellipse_n \\
\hline 388 & $*$ & satisfiability_n & 428 & $*$ & halt_n \\
\hline 389 & $*$ & menu_n & 429 & & fork_n \\
\hline 390 & $*$ & disjunction_n & 430 & & locally_adv \\
\hline 391 & $*$ & lock_adj & 431 & & grade_n \\
\hline 392 & & combine_n & 432 & & gender_n \\
\hline 393 & & complicate_v & 433 & & dissemination_n \\
\hline 394 & & differentiate_v & 434 & & disclosure_n \\
\hline 395 & & compete_v & 435 & & constituent_adj \\
\hline 396 & & dense_adj & 436 & & surprising_adj \\
\hline 397 & & successive_adj & 437 & $*$ & index_v \\
\hline 398 & & caller_n & 438 & & ambiguity_n \\
\hline 399 & & engineer_v & 439 & & initiate_v \\
\hline 400 & & battery_n & 440 & & popularity_n \\
\hline 401 & & questionnaire_n & 441 & $\#$ & intersect_v \\
\hline 402 & & migrate_v & 442 & & compress_v \\
\hline 403 & & lottery_n & 443 & $*$ & zone_n \\
\hline 404 & & double_n & 444 & $*$ & initialization_n \\
\hline 405 & & fetch_n & 445 & & incomplete_adj \\
\hline 406 & $*$ & inverse_adj & 446 & & accounting_n \\
\hline 407 & & converge_v & 447 & & viewpoint_n \\
\hline 408 & & slide_v & 448 & $*$ & heuristic_n \\
\hline 409 & & enhancement_n & 449 & & custom_n \\
\hline 410 & & informal_adj & 450 & & audio_adj \\
\hline 411 & & synthesis_n & 451 & & batch_n \\
\hline 412 & & cue_n & 452 & & parse_v \\
\hline
\end{tabular}




\begin{tabular}{|c|c|c|c|c|c|}
\hline 453 & & domino_n & 493 & $*$ & median_adj \\
\hline 454 & & forwarding_n & 494 & & topological_adj \\
\hline 455 & $*$ & unlock_v & 495 & $*$ & linkage_n \\
\hline 456 & $*$ & spreadsheet_n & 496 & & geographic_adj \\
\hline 457 & & reasonably_adv & 497 & $\#$ & singular_adj \\
\hline 458 & & administrator_n & 498 & $\#$ & weight_v \\
\hline 459 & & reconstruct_v & 499 & & usable_adj \\
\hline 460 & & lab_n & 500 & & free_v \\
\hline 461 & & short_adv & 501 & $*$ & segment_v \\
\hline 462 & & autonomous_adj & 502 & & skeletal_adj \\
\hline 463 & & speedup_n & 503 & & tweet_v \\
\hline 464 & & timer_n & 504 & & clique_n \\
\hline 465 & & leakage_n & 505 & & recognizable_adj \\
\hline 466 & & strain_n & 506 & & predefined_adj \\
\hline 467 & $*$ & desktop_n & 507 & & accommodate_v \\
\hline 468 & & substitute_v & 508 & & ease_n \\
\hline 469 & & synthesize_v & 509 & & visibility_n \\
\hline 470 & $*$ & corrupt_v & 510 & $*$ & radix_n \\
\hline 471 & & procedural_adj & 511 & $\#$ & focal_adj \\
\hline 472 & $*$ & terminal_n & 512 & $*$ & object_adj \\
\hline 473 & $\#$ & convert_n & 513 & $\#$ & die_n \\
\hline 474 & $*$ & inheritance_n & 514 & & compensate_v \\
\hline 475 & $*$ & traversal_adj & 515 & & skip_v \\
\hline 476 & & federate_v & 516 & $*$ & pack_v \\
\hline 477 & & checkerboard_n & 517 & & unexpected_adj \\
\hline 478 & & convenience_n & 518 & & equal_n \\
\hline 479 & & objective_adj & 519 & & degradation_n \\
\hline 480 & & degrade_v & 520 & & decrypt_v \\
\hline 481 & $*$ & bias_v & 521 & & instrumentation_n \\
\hline 482 & $*$ & clustering_n & 522 & & override_v \\
\hline 483 & $*$ & layer_v & 523 & & reverse_v \\
\hline 484 & & implement_n & 524 & & visualize_v \\
\hline 485 & & dependability_n & 525 & & equip_v \\
\hline 486 & & unroll_v & 526 & & inconsistency_n \\
\hline 487 & & subsequently_adv & 527 & $\#$ & integral_adj \\
\hline 488 & $*$ & isolation_n & 528 & & mutually_adv \\
\hline 489 & & indirect_adj & 529 & & concatenate_v \\
\hline 490 & $\#$ & aggregate_adj & 530 & & conceptual_adj \\
\hline 491 & $\#$ & pair_v & 531 & $*$ & intensity_n \\
\hline 492 & & preprocessing_n & 532 & $*$ & microprocessor_n \\
\hline
\end{tabular}




\begin{tabular}{|c|c|c|c|c|c|}
\hline 533 & $*$ & sentinel_n & 573 & & inspection_n \\
\hline 534 & & alternate_adj & 574 & & cryptographic_adj \\
\hline 535 & & millisecond_n & 575 & & satisfiable_adj \\
\hline 536 & & targeted_adj & 576 & $*$ & verifier_n \\
\hline 537 & $*$ & transistor_n & 577 & & subtle_adj \\
\hline 538 & & infinitely_adv & 578 & & analogous_adj \\
\hline 539 & & interconnect_v & 579 & & competitive_adj \\
\hline 540 & & nonlinear_adj & 580 & & publicly_adv \\
\hline 541 & $*$ & payload_n & 581 & & iteratively_adv \\
\hline 542 & & alert_n & 582 & & contiguous_adj \\
\hline 543 & & third_n & 583 & & parenthesis_n \\
\hline 544 & & particular_n & 584 & $\#$ & diameter_n \\
\hline 545 & & advance_v & 585 & & subjective_adj \\
\hline 546 & $\#$ & equivalent_n & 586 & $*$ & primitive_n \\
\hline 547 & & contradict_v & 587 & $*$ & cryptography_n \\
\hline 548 & & repetition_n & 588 & & thread_v \\
\hline 549 & $*$ & pop_n & 589 & $*$ & substring_n \\
\hline 550 & $\#$ & logarithm_n & 590 & $*$ & cookie_n \\
\hline 551 & & sender_n & 591 & & regularization_n \\
\hline 552 & $*$ & chain_v & 592 & & asymptotically_adv \\
\hline 553 & & predecessor_n & 593 & $*$ & cybercrime_n \\
\hline 554 & & fitness_n & 594 & & holographic_adj \\
\hline 555 & & blockchain_n & 595 & $*$ & bound_adj \\
\hline 556 & & microstructure_n & 596 & $*$ & network_v \\
\hline 557 & & differently_adv & 597 & $*$ & counter_v \\
\hline 558 & & aforementioned_adj & 598 & & reflection_n \\
\hline 559 & $*$ & dynamic_n & 599 & & prune_v \\
\hline 560 & & suffice_v & 600 & & duplicate_n \\
\hline 561 & & simulated_adj & 601 & $*$ & cardinality_n \\
\hline 562 & & quantitative_adj & 602 & & noun_n \\
\hline 563 & $\#$ & replication_n & 603 & $*$ & continuation_n \\
\hline 564 & & resume_v & 604 & & instructor_n \\
\hline 565 & & sparsity_n & 605 & $*$ & atom_n \\
\hline 566 & $*$ & keyboard_n & 606 & & stimulus_n \\
\hline 567 & & predictive_adj & 607 & & tetrahedral_adj \\
\hline 568 & & forward_v & 608 & & tradeoff_n \\
\hline 569 & & remark_n & 609 & $*$ & tag_v \\
\hline 570 & & fill_n & 610 & & iterate_v \\
\hline 571 & $\#$ & friction_n & 611 & $\#$ & gradient_adj \\
\hline 572 & $\#$ & compact_adj & 612 & & privileged_adj \\
\hline
\end{tabular}




\begin{tabular}{|c|c|c|c|c|c|}
\hline 613 & & technological_adj & 653 & $*$ & tile_v \\
\hline 614 & & inefficient_adj & 654 & $*$ & actuator_n \\
\hline 615 & & combined_adj & 655 & & diffusion_n \\
\hline 616 & & media_n & 656 & $*$ & residual_adj \\
\hline 617 & $*$ & certification_n & 657 & & neural_adj \\
\hline 618 & & audit_n & 658 & $\#$ & proportion_n \\
\hline 619 & & spectrum_n & 659 & & adjacency_n \\
\hline 620 & & prohibit_v & 660 & & virtualized_adj \\
\hline 621 & $*$ & crash_n & 661 & & dialog_n \\
\hline 622 & $*$ & dedicated_adj & 662 & $*$ & reconfiguration_n \\
\hline 623 & & algorithmic_adj & 663 & & automobile_n \\
\hline 624 & & symbolic_adj & 664 & & retention_n \\
\hline 625 & & federal_adj & 665 & & categorical_adj \\
\hline 626 & & sensitivity_n & 666 & & backward_adv \\
\hline 627 & & exploration_n & 667 & & reachable_adj \\
\hline 628 & $*$ & tower_n & 668 & & mismatch_n \\
\hline 629 & & aberration_n & 669 & & power_v \\
\hline 630 & & activation_n & 670 & $\#$ & angular_adj \\
\hline 631 & & exponentially_adv & 671 & $\#$ & cylinder_n \\
\hline 632 & & planar_adj & 672 & & breach_n \\
\hline 633 & & exit_v & 673 & & microphone_n \\
\hline 634 & $*$ & spread_n & 674 & & mutation_n \\
\hline 635 & & tune_v & 675 & & projector_n \\
\hline 636 & & ranking_n & 676 & & scattering_n \\
\hline 637 & & repeated_adj & 677 & & catalog_n \\
\hline 638 & & automation_n & 678 & $\#$ & pairwise_adj \\
\hline 639 & $*$ & checkpoint_n & 679 & & robot_n \\
\hline 640 & $*$ & coupling_n & 680 & $*$ & assembler_n \\
\hline 641 & & passive_adj & 681 & $*$ & modulation_n \\
\hline 642 & & antenna_n & 682 & & magnification_n \\
\hline 643 & & incentive_n & 683 & & predictor_n \\
\hline 644 & & orthogonal_adj & 684 & & bipartite_adj \\
\hline 645 & $\#$ & exhaustive_adj & 685 & $\#$ & observer_n \\
\hline 646 & & accumulate_v & 686 & $\#$ & factorial_adj \\
\hline 647 & $*$ & token_adj & 687 & & theoretic_adj \\
\hline 648 & $\#$ & isolate_v & 688 & $*$ & serial_adj \\
\hline 649 & & formalize_v & 689 & & stem_n \\
\hline 650 & $*$ & transparent_adj & 690 & $\#$ & equilibrium_n \\
\hline 651 & & shrink_v & 691 & & crack_v \\
\hline 652 & & privilege_n & 692 & $\#$ & pyramid_n \\
\hline
\end{tabular}


A COMPUTER SCIENCE ACADEMIC VOCABULARY LIST

$\begin{array}{ll}693 & \text { analytic_n } \\ 694 & \text { emit_v } \\ 695 & \text { lawsuit_n } \\ 696 & \text { analog_adj } \\ 697 & \text { isotropic_adj }\end{array}$

$\begin{array}{lll}698 & * & \text { benchmark_adj } \\ 699 & & \text { interpretable_adj } \\ 700 & * & \text { tab_n } \\ 701 & \# & \text { factorial_n } \\ 702 & * & \text { screen_v }\end{array}$




\section{Appendix C: Textbook Usage Survey Data}

Table C.1 Textbook usage survey results

\begin{tabular}{|c|c|c|c|c|c|c|}
\hline $\begin{array}{l}\text { Mean Rank } \\
\text { (Usnews / } \\
\text { CSrankings) }\end{array}$ & Institution & Core CS course name & $\begin{array}{l}\text { Text } \\
\text { status }\end{array}$ & Category & Title & Authors \\
\hline \multirow[t]{12}{*}{$1(1 / 1)$} & \multirow[t]{12}{*}{$\begin{array}{l}\text { Carnegie } \\
\text { Mellon }\end{array}$} & $\begin{array}{l}\text { 15-122: Principles of } \\
\text { Imperative } \\
\text { Computation }\end{array}$ & NA & prog & NA & NA \\
\hline & & $\begin{array}{l}\text { 15-150: Principles of } \\
\text { Functional } \\
\text { Programming }\end{array}$ & required & prog & $\begin{array}{l}\text { Programming in } \\
\text { Standard ML }\end{array}$ & Robert Harper \\
\hline & & \multirow[t]{2}{*}{$\begin{array}{l}\text { 15-151: Mathematical } \\
\text { Foundations for } \\
\text { Computer Science }\end{array}$} & optional & math & $\begin{array}{l}\text { Everything You Always } \\
\text { Wanted to Know About } \\
\text { Mathematics }\end{array}$ & Brendan Sullivan \\
\hline & & & optional & math & $\begin{array}{l}\text { Mathematical Thinking: } \\
\text { Problem-Solving and } \\
\text { Proofs }\end{array}$ & D'Angelo and West \\
\hline & & $\begin{array}{l}\text { 15-210: Parallel and } \\
\text { Sequential Data } \\
\text { Structures and } \\
\text { Algorithms }\end{array}$ & required & alg & $\begin{array}{l}\text { Algorithms: Parallel } \\
\text { and Sequential }\end{array}$ & $\begin{array}{l}\text { Umut A. Acar and } \\
\text { Guy E. Blelloch }\end{array}$ \\
\hline & & \multirow[t]{2}{*}{$\begin{array}{l}\text { 15-213: Introduction to } \\
\text { Computer Systems }\end{array}$} & required & sys_org & $\begin{array}{l}\text { Computer Systems: A } \\
\text { Programmer's } \\
\text { Perspective }\end{array}$ & $\begin{array}{l}\text { Randal E. Bryant } \\
\text { and David R. } \\
\text { O'Hallaron }\end{array}$ \\
\hline & & & required & prog & $\begin{array}{l}\text { The C Programming } \\
\text { Language }\end{array}$ & $\begin{array}{l}\text { Brian Kernighan and } \\
\text { Dennis Ritchie }\end{array}$ \\
\hline & & \multirow[t]{4}{*}{$\begin{array}{l}\text { 15-251: Great Ideas in } \\
\text { Theoretical Computer } \\
\text { Science }\end{array}$} & optional & theory & $\begin{array}{l}\text { Introduction to the } \\
\text { Theory of Computation }\end{array}$ & Sipser, Michael. \\
\hline & & & optional & theory & $\begin{array}{l}\text { The Nature of } \\
\text { Computation }\end{array}$ & $\begin{array}{l}\text { Cristopher Moore } \\
\text { and Stephan Mertens }\end{array}$ \\
\hline & & & optional & theory & $\begin{array}{l}\text { Introduction to } \\
\text { Theoretical Computer } \\
\text { Science }\end{array}$ & Boaz Barak \\
\hline & & & optional & theory & $\begin{array}{l}\text { Quantum Computing } \\
\text { Since Democritus }\end{array}$ & Scott Aaronson \\
\hline & & $\begin{array}{l}\text { 15-451: Algorithm } \\
\text { Design and Analysis }\end{array}$ & NA & alg & NA & NA \\
\hline \multirow[t]{3}{*}{$1.5(1 / 2)$} & \multirow[t]{3}{*}{$\mathrm{MIT}$} & $\begin{array}{l}\text { 6.0001 Introduction to } \\
\text { Computer Science } \\
\text { Programming in Python }\end{array}$ & required & prog & $\begin{array}{l}\text { Introduction to } \\
\text { Computation and } \\
\text { Programming Using } \\
\text { Python: With } \\
\text { Application to } \\
\text { Understanding Data }\end{array}$ & Guttag, John \\
\hline & & $\begin{array}{l}\text { 6.004 Computation } \\
\text { Structures }\end{array}$ & optional & sys_org & $\begin{array}{l}\text { Computer Architecture: } \\
\text { A Constructive } \\
\text { Approach. }\end{array}$ & $\begin{array}{l}\text { Arvind, Rishiyur } \\
\text { Nikhil, Joel Emer, } \\
\text { Murali } \\
\text { Vijayaraghavan }\end{array}$ \\
\hline & & & optional & sys_org & $\begin{array}{l}\text { Digital Design: A } \\
\text { Systems Approach. }\end{array}$ & $\begin{array}{l}\text { William Dally and R } \\
\text { Curtis Harting. }\end{array}$ \\
\hline
\end{tabular}




\begin{tabular}{|c|c|c|c|c|c|c|}
\hline & & \multirow[b]{2}{*}{$\begin{array}{l}\text { 6.006 Introduction to } \\
\text { Algorithms }\end{array}$} & optional & sys_org & \multirow{2}{*}{$\begin{array}{l}\text { Computer Organization } \\
\text { and Design: The } \\
\text { Hardware Software } \\
\text { Interface (RISC-V } \\
\text { Edition). } \\
\text { Introduction to } \\
\text { Algorithms. }\end{array}$} & $\begin{array}{l}\text { David Patterson and } \\
\text { John Hennessy. }\end{array}$ \\
\hline & & & required & alg & & $\begin{array}{l}\text { Cormen, Thomas, } \\
\text { Charles Leiserson, } \\
\text { Ronald Rivest, and } \\
\text { Clifford Stein }\end{array}$ \\
\hline & & \multirow[t]{4}{*}{$\begin{array}{l}\text { 6.009 Fundamentals of } \\
\text { Programming }\end{array}$} & optional & prog & Debug It! & Paul Butcher \\
\hline & & & optional & prog & Debugging & David J. Agans \\
\hline & & & optional & prog & Why Programs Fail & $\begin{array}{l}\text { Andreas Zeller, } \\
\text { Morgan Kaufmann }\end{array}$ \\
\hline & & & optional & prog & The Python Tutorial & $\begin{array}{l}\text { The Python Software } \\
\text { Foundation }\end{array}$ \\
\hline & & $\begin{array}{l}\text { 6.031 Elements of } \\
\text { Software Construction }\end{array}$ & NA & soft_design & NA & NA \\
\hline & & $\begin{array}{l}\text { 6.033 Computer } \\
\text { Systems Engineering } \\
\text { (CI-M) }\end{array}$ & required & sys_org & $\begin{array}{l}\text { Principles of Computer } \\
\text { System Design: An } \\
\text { Introduction, Part I. } \\
\text { Principles of Computer } \\
\text { System Design: An } \\
\text { Introduction, Part II. }\end{array}$ & $\begin{array}{l}\text { Saltzer, Jerome H. } \\
\text { and M. Frans } \\
\text { Kaashoek. Morgan } \\
\text { Kaufmann, } \\
\text { Saltzer, Jerome H. } \\
\text { and M. Frans } \\
\text { Kaashoek. Morgan } \\
\text { Kaufmann, }\end{array}$ \\
\hline & & $\begin{array}{l}\text { 6.034 Artificial } \\
\text { Intelligence }\end{array}$ & required & & Artificial Intelligence & $\begin{array}{l}\text { Patrick Henry } \\
\text { Winston (MIT) }\end{array}$ \\
\hline & & $\begin{array}{l}\text { 6.036 Introduction to } \\
\text { Machine Learning }\end{array}$ & NA & & NA & NA \\
\hline & & \multirow[t]{3}{*}{$\begin{array}{l}6.045[\mathrm{~J}] \text { Automata, } \\
\text { Computability, and } \\
\text { Complexity }\end{array}$} & optional & theory & $\begin{array}{l}\text { The Nature of } \\
\text { Computation }\end{array}$ & $\begin{array}{l}\text { Moore, Cristopher, } \\
\text { and Stephan } \\
\text { Mertens. }\end{array}$ \\
\hline & & & optional & theory & $\begin{array}{l}\text { Introduction to the } \\
\text { Theory of Computation }\end{array}$ & Sipser, Michael. \\
\hline & & & optional & theory & $\begin{array}{l}\text { Computational } \\
\text { Complexity: A Modern } \\
\text { Approach. }\end{array}$ & $\begin{array}{l}\text { Arora, Sanjeev, and } \\
\text { Boaz Barak. }\end{array}$ \\
\hline & & $\begin{array}{l}6.046[\mathrm{~J}] \text { Design and } \\
\text { Analysis of Algorithms }\end{array}$ & required & alg & $\begin{array}{l}\text { Introduction to } \\
\text { Algorithms }\end{array}$ & $\begin{array}{l}\text { Cormen, Thomas H.; } \\
\text { Leiserson, Charles } \\
\text { E.; Rivest, Ronald } \\
\text { L.; Stein, Clifford }\end{array}$ \\
\hline \multirow[t]{3}{*}{$2(1 / 3)$} & Stanford & $\begin{array}{l}\text { Programming } \\
\text { Abstractions (CS106B } \\
\text { or CS106X) }\end{array}$ & required & prog & $\begin{array}{l}\text { Programming } \\
\text { Abstractions in } \mathrm{C}++ \text {. }\end{array}$ & Roberts, Eric S. \\
\hline & \multirow[t]{2}{*}{-} & \multirow[t]{2}{*}{$\begin{array}{l}\text { Computer Organization } \\
\text { and Systems (CS107) }\end{array}$} & required & sys_org & Computer Systems & $\begin{array}{l}\text { Bryant and } \\
\text { O'Hallaron }\end{array}$ \\
\hline & & & required & prog & $\begin{array}{l}\text { C Programming } \\
\text { Language }\end{array}$ & $\begin{array}{l}\text { Brian Kernighan and } \\
\text { Dennis Ritchie }\end{array}$ \\
\hline
\end{tabular}




\begin{tabular}{|c|c|c|c|c|c|c|}
\hline & & & required & prog & Essential C & Nick Parlante \\
\hline & & $\begin{array}{l}\text { Principles of Computer } \\
\text { Systems (CS110) }\end{array}$ & required & sys_org & Computer Systems & $\begin{array}{l}\text { Bryant and } \\
\text { O'Hallaron }\end{array}$ \\
\hline & & & required & sys_org & $\begin{array}{l}\text { Principles of Computer } \\
\text { System Design: An } \\
\text { Introduction }\end{array}$ & $\begin{array}{l}\text { Jerome H. Saltzer } \\
\text { and M. Frans } \\
\text { Kaashoek }\end{array}$ \\
\hline & & $\begin{array}{l}\text { Mathematical } \\
\text { Foundations of } \\
\text { Computing (CS103) }\end{array}$ & optional & math & $\begin{array}{l}\text { How to Read and Do } \\
\text { Proofs }\end{array}$ & Daniel Solow \\
\hline & & & optional & theory & $\begin{array}{l}\text { Introduction to the } \\
\text { Theory of Computation }\end{array}$ & Sipser, Michael. \\
\hline & & $\begin{array}{l}\text { Introduction to } \\
\text { Probability for } \\
\text { Computer } \\
\text { Scientists (CS109) }\end{array}$ & required & prob_stats & NA & NA \\
\hline & & $\begin{array}{l}\text { Data Structures and } \\
\text { Algorithms (CS161) }\end{array}$ & required & alg & $\begin{array}{l}\text { Introduction to } \\
\text { Algorithms }\end{array}$ & $\begin{array}{l}\text { Thomas H. Cormen, } \\
\text { Charles E. Leiserson, } \\
\text { Ronald L. Rivest, } \\
\text { Clifford Stein }\end{array}$ \\
\hline & & & optional & alg & Algorithm Design & $\begin{array}{l}\text { Jon Kleinberg, Éva } \\
\text { Tardos }\end{array}$ \\
\hline & & & $\begin{array}{l}\text { optional } \\
\text { optional }\end{array}$ & alg & $\begin{array}{l}\text { Algorithms } \\
\text { Algorithms Illuminated }\end{array}$ & $\begin{array}{l}\text { Sanjoy Dasgupta, } \\
\text { Christos } \\
\text { Papadimitriou, } \\
\text { Umesh Vaziran } \\
\text { Tim Roughgarden }\end{array}$ \\
\hline \multirow[t]{9}{*}{$3(1 / 5)$} & $\begin{array}{l}\text { University of } \\
\text { California- } \\
\text { Berkeley }\end{array}$ & $\begin{array}{l}\text { COMPSCI 61A The } \\
\text { Structure and } \\
\text { Interpretation of } \\
\text { Computer Programs }\end{array}$ & required & prog & $\begin{array}{l}\text { Structure and } \\
\text { Interpretation of } \\
\text { Computer Programs }\end{array}$ & $\begin{array}{l}\text { Hal Abelson, Jerry } \\
\text { Sussman and Julie } \\
\text { Sussman }\end{array}$ \\
\hline & & $\begin{array}{l}\text { COMPSCI 61B Data } \\
\text { Structures }\end{array}$ & optional & prog & Head First Java & Sierra and Bates \\
\hline & & & required & data_struc & NA & NA \\
\hline & & & required & data_struc & NA & NA \\
\hline & & & optional & alg & Algorithms & $\begin{array}{l}\text { Robert Sedgewick } \\
\text { and Kevin Wayne }\end{array}$ \\
\hline & \multirow[t]{4}{*}{-} & $\begin{array}{l}\text { COMPSCI } 70 \text { Discrete } \\
\text { Mathematics and } \\
\text { Probability Theory }\end{array}$ & NA & math & NA & NA \\
\hline & & $\begin{array}{l}\text { COMPSCI 61C Great } \\
\text { Ideas of Computer } \\
\text { Architecture (Machine } \\
\text { Structures) }\end{array}$ & required & sys_org & $\begin{array}{l}\text { Computer Organization } \\
\text { and Design RISC-V } \\
\text { Edition }\end{array}$ & $\begin{array}{l}\text { David Patterson, and } \\
\text { John Hennessy }\end{array}$ \\
\hline & & & required & prog & $\begin{array}{l}\text { The C Programming } \\
\text { Language }\end{array}$ & $\begin{array}{l}\text { Brian Kernighan and } \\
\text { Dennis Ritchie }\end{array}$ \\
\hline & & & required & sys_org & $\begin{array}{l}\text { The Datacenter as a } \\
\text { Computer }\end{array}$ & $\begin{array}{l}\text { Luiz André Barroso } \\
\text { and Urs Hölzle }\end{array}$ \\
\hline
\end{tabular}




\begin{tabular}{|c|c|c|c|c|c|c|}
\hline \multirow[t]{19}{*}{$4.5(5 / 4)$} & $\begin{array}{l}\text { University of } \\
\text { Illinois } \\
\text { Urbana- } \\
\text { Champaign }\end{array}$ & $\begin{array}{l}\text { CS } 125 \text { Introduction to } \\
\text { Computer Science }\end{array}$ & required & human & Coders & Clive Thompson \\
\hline & - & $\begin{array}{l}\text { CS } 126 \text { Software } \\
\text { Design Studio }\end{array}$ & required & soft_design & $\begin{array}{l}\text { The Art of Readable } \\
\text { Code }\end{array}$ & $\begin{array}{l}\text { Dustin Boswell, } \\
\text { Trevor Foucher }\end{array}$ \\
\hline & & $\begin{array}{l}\text { CS } 173 \text { Discrete } \\
\text { Structures }\end{array}$ & required & math & $\begin{array}{l}\text { Mathematics for } \\
\text { Computer Science }\end{array}$ & $\begin{array}{l}\text { Eric Lehman, Tom } \\
\text { Leighton, and Albert } \\
\text { Meyers }\end{array}$ \\
\hline & & & optional & theory & $\begin{array}{l}\text { Building Blocks for } \\
\text { Theoretical Computer } \\
\text { Science }\end{array}$ & Margaret Fleck \\
\hline & & $\begin{array}{l}\text { CS } 210 \text { Ethical and } \\
\text { Professional Issues in } \\
\text { CS }\end{array}$ & NA & human & NA & NA \\
\hline & & $\begin{array}{l}\text { CS } 225 \text { Data Structure } \\
\text { and Software Principles }\end{array}$ & NA & data_struc & NA & NA \\
\hline & & $\begin{array}{l}\text { CS } 233 \text { Computer } \\
\text { Architecture }\end{array}$ & required & sys_org & $\begin{array}{l}\text { Logic and Computer } \\
\text { Design Fundamentals }\end{array}$ & $\begin{array}{l}\text { M. Morris Mano and } \\
\text { Charles R. Kime }\end{array}$ \\
\hline & & & optional & sys_org & $\begin{array}{l}\text { Computer Organization } \\
\text { \& Design: The } \\
\text { Hardware/Software } \\
\text { Interface }\end{array}$ & $\begin{array}{l}\text { David A. Patterson } \\
\text { and John L. } \\
\text { Hennessy. }\end{array}$ \\
\hline & & & optional & sys_org & $\begin{array}{l}\text { Verilog HDL: A Guide } \\
\text { to Digital Design and } \\
\text { Synthesis }\end{array}$ & Samir Palnitkar \\
\hline & & $\begin{array}{l}\text { CS } 241 \text { System } \\
\text { Programming }\end{array}$ & required & sys_org & NA & NA \\
\hline & & $\begin{array}{l}\text { CS } 357 \text { Numerical } \\
\text { Methods I }\end{array}$ & optional & math & $\begin{array}{l}\text { Numerical Mathematics } \\
\text { and Computing }\end{array}$ & Cheney and Kinkaid \\
\hline & & $\begin{array}{l}\text { CS } 361 \text { Probability and } \\
\text { Statistics for Computer } \\
\text { Science }\end{array}$ & required & prob_stats & $\begin{array}{l}\text { Probability and } \\
\text { Statistics for Computer } \\
\text { Science }\end{array}$ & Forsyth, D. A. \\
\hline & & $\begin{array}{l}\text { CS } 374 \text { Algorithms and } \\
\text { Models of Computation }\end{array}$ & required & alg & Algorithms & Jeff Erickson \\
\hline & & $\begin{array}{l}\text { CS } 421 \text { Programming } \\
\text { Languages and } \\
\text { Compilers }\end{array}$ & optional & prog & $\begin{array}{l}\text { The Objective Caml } \\
\text { system }\end{array}$ & Xavier Leroy \\
\hline & & & optional & prog & $\begin{array}{l}\text { Introduction to } \\
\text { Objective Caml }\end{array}$ & Jason Hickey \\
\hline & & & optional & prog & $\begin{array}{l}\text { Modern Compiler } \\
\text { Implementation in ML }\end{array}$ & Andrew Appel \\
\hline & & & optional & prog & $\begin{array}{l}\text { Compilers: Principles, } \\
\text { Techniques, and Tools, } \\
\text { also known as "The } \\
\text { Dragon Book" }\end{array}$ & $\begin{array}{l}\text { Aho, Sethi, and } \\
\text { Ullman. }\end{array}$ \\
\hline & & & optional & prog & $\begin{array}{l}\text { Essentials of } \\
\text { Programming } \\
\text { Languages }\end{array}$ & $\begin{array}{l}\text { Friedman, Wand, } \\
\text { and Haynes }\end{array}$ \\
\hline & & & optional & prog & $\begin{array}{l}\text { Advanced } \\
\text { Programming Language } \\
\text { Design }\end{array}$ & Raphael A. Finkel \\
\hline
\end{tabular}




\begin{tabular}{|c|c|c|c|c|c|c|}
\hline & & & \multirow{3}{*}{$\begin{array}{l}\text { optional } \\
\text { optional }\end{array}$} & \multirow{3}{*}{$\begin{array}{l}\text { prog } \\
\text { prog }\end{array}$} & \multirow{3}{*}{$\begin{array}{l}\text { Programming Language } \\
\text { Pragmatics } \\
\text { Concepts, Techniques, } \\
\text { and Models of } \\
\text { Computer Programming }\end{array}$} & \multirow{3}{*}{$\begin{array}{l}\text { Michael L. Scott } \\
\text { Peter Van Roy and } \\
\text { Seif Haridi }\end{array}$} \\
\hline & & & & & & \\
\hline & & & & & & \\
\hline \multirow[t]{15}{*}{$6(6 / 6)$} & Cornell & $\begin{array}{l}\text { CS 111x (CS } 1110 \text { Intro } \\
\text { to Computing with } \\
\text { Python, 1112, 1114, or } \\
1115)\end{array}$ & required & prog & $\begin{array}{l}\text { Think Python: How to } \\
\text { Think Like a Computer } \\
\text { Scientist }\end{array}$ & Allen B. Downey \\
\hline & - & $\begin{array}{l}\text { CS } 2110 \text { (or CS 2112) } \\
\text { OO Programming \& } \\
\text { Data Structures }\end{array}$ & NA & data_struc & $\begin{array}{l}\text { JavaHyperText and } \\
\text { Data Structures }\end{array}$ & $\begin{array}{l}\text { David Gries, with } \\
\text { help from many }\end{array}$ \\
\hline & & $\begin{array}{l}\text { CS } 2800 \text { Discrete } \\
\text { Structures (or CS 2802) }\end{array}$ & required & math & $\begin{array}{l}\text { Mathematics for } \\
\text { Computer Science } \\
\text { (MCS) }\end{array}$ & $\begin{array}{l}\text { Lehman, Leighton, } \\
\text { and Meyer. }\end{array}$ \\
\hline & & & optional & math & $\begin{array}{l}\text { A Course in Discrete } \\
\text { Structures }\end{array}$ & $\begin{array}{l}\text { Rafael Pass Wei- } \\
\text { Lung Dustin Tseng }\end{array}$ \\
\hline & & $\begin{array}{l}\text { CS } 3110 \text { Data } \\
\text { Structures and } \\
\text { Functional } \\
\text { Programming }\end{array}$ & required & prog & $\begin{array}{l}\text { Functional } \\
\text { Programming in Ocaml }\end{array}$ & $\begin{array}{l}\text { Michael R. Clarkson, } \\
\text { Robert L. Constable, } \\
\text { Nate Foster, Michael } \\
\text { D. George, Dan } \\
\text { Grossman, Daniel P. } \\
\text { Huttenlocher, Dexter } \\
\text { Kozen, Greg } \\
\text { Morrisett, Andrew } \\
\text { C. Myers, Radu } \\
\text { Rugina, and Ramin } \\
\text { Zabih }\end{array}$ \\
\hline & & $\begin{array}{l}\text { CS } 3410 \text { Computer } \\
\text { System Organization } \\
\text { and Programming }\end{array}$ & required & sys_org & $\begin{array}{l}\text { Computer Organization } \\
\text { and Design RISC-V } \\
\text { Edition }\end{array}$ & $\begin{array}{l}\text { David A. } \\
\text { Patterson , John L. } \\
\text { Hennessy }\end{array}$ \\
\hline & & & optional & op_sys & Linux Pocket Guide & Daniel J. Barrett \\
\hline & & & optional & prog & All of Programming & $\begin{array}{l}\text { Andrew Hilton Anne } \\
\text { Bracy }\end{array}$ \\
\hline & & $\begin{array}{l}\text { CS } 4410 \text { Operating } \\
\text { Systems }\end{array}$ & required & op_sys & $\begin{array}{l}\text { Operating Systems: } \\
\text { Three Easy Pieces }\end{array}$ & $\begin{array}{l}\text { Remzi H. Arpaci- } \\
\text { Dusseau and Andrea } \\
\text { C. Arpaci-Dusseau. }\end{array}$ \\
\hline & & & optional & op_sys & $\begin{array}{l}\text { Operating Systems: } \\
\text { Principles and Practice }\end{array}$ & $\begin{array}{l}\text { Tom Anderson and } \\
\text { Mike Dahlin }\end{array}$ \\
\hline & & $\begin{array}{l}\text { CS } 4820 \text { Introduction to } \\
\text { Analysis of Algorithms }\end{array}$ & required & alg & Algorithm Design & $\begin{array}{l}\text { Jon Kleinberg and } \\
\text { Eva Tardos }\end{array}$ \\
\hline & & & optional & alg & $\begin{array}{l}\text { Introduction to } \\
\text { Algorithms }\end{array}$ & $\begin{array}{l}\text { T. Cormen, C. } \\
\text { Leiserson, R. Rivest }\end{array}$ \\
\hline & & & optional & alg & Algorithms & $\begin{array}{l}\text { S. Dasgupta, C. } \\
\text { Papadimitriou, and } \\
\text { U. Vazirani }\end{array}$ \\
\hline & & & optional & alg & $\begin{array}{l}\text { The Design and } \\
\text { Analysis of Computer } \\
\text { Algorithms }\end{array}$ & $\begin{array}{l}\text { A. Aho, J. Hopcroft, } \\
\text { J. Ullman }\end{array}$ \\
\hline & & & optional & alg & $\begin{array}{l}\text { Computers and } \\
\text { Intractability }\end{array}$ & $\begin{array}{l}\text { M. Garey and D. } \\
\text { Johnson }\end{array}$ \\
\hline
\end{tabular}




\begin{tabular}{|c|c|c|c|c|c|c|}
\hline & & & optional & alg & $\begin{array}{l}\text { The Design and } \\
\text { Analysis of Algorithms }\end{array}$ & D. Kozen \\
\hline \multirow[t]{11}{*}{$6.5(6 / 7)$} & $\begin{array}{l}\text { University of } \\
\text { Washington }\end{array}$ & $\begin{array}{l}\text { CSE } 142 \text { Computer } \\
\text { Programming I }\end{array}$ & required & prog & $\begin{array}{l}\text { Building Java } \\
\text { Programs: A Back to } \\
\text { Basics Approach }\end{array}$ & Reges/Stepp \\
\hline & - & $\begin{array}{l}\text { CSE } 143 \text { Computer } \\
\text { Programming II }\end{array}$ & required & prog & $\begin{array}{l}\text { Building Java } \\
\text { Programs: A Back to } \\
\text { Basics Approach }\end{array}$ & Reges/Stepp \\
\hline & & $\begin{array}{l}\text { CSE } 311 \text { Foundations } \\
\text { of Computing I }\end{array}$ & optional & math & $\begin{array}{l}\text { Discrete Mathematics } \\
\text { and Its Applications }\end{array}$ & Kenneth Rosen \\
\hline & & $\begin{array}{l}\text { CSE } 312 \text { Foundations } \\
\text { of Computing II }\end{array}$ & required & prob_stats & $\begin{array}{l}\text { Introduction to } \\
\text { Probability }\end{array}$ & $\begin{array}{l}\text { Dimitri P. Bertsekas } \\
\text { and John N. } \\
\text { Tsitsiklis }\end{array}$ \\
\hline & & $\begin{array}{l}\text { CSE } 331 \text { Software } \\
\text { Design \& } \\
\text { Implementation }\end{array}$ & required & prog & Effective Java & Joshua Bloch \\
\hline & & & required & prog & $\begin{array}{l}\text { The Pragmatic } \\
\text { Programmer }\end{array}$ & $\begin{array}{l}\text { Andrew Hunt and } \\
\text { David Thomas }\end{array}$ \\
\hline & & & optional & prog & $\begin{array}{l}\text { Program Development } \\
\text { in Java }\end{array}$ & $\begin{array}{l}\text { Barbara Liskov and } \\
\text { John Guttag }\end{array}$ \\
\hline & & $\begin{array}{l}\text { CSE } 332 \text { Data } \\
\text { Abstractions }\end{array}$ & required & data_struc & $\begin{array}{l}\text { Data Structures and } \\
\text { Algorithm Analysis in } \\
\text { Java }\end{array}$ & Mark Allen Weiss \\
\hline & & $\begin{array}{l}\text { CSE } 351 \text { The } \\
\text { Hardware/Software } \\
\text { Interface }\end{array}$ & required & sys_org & $\begin{array}{l}\text { Computer Systems: A } \\
\text { Programmer's } \\
\text { Perspective }\end{array}$ & $\begin{array}{l}\text { Randal E. Bryant } \\
\text { and David R. } \\
\text { O'Hallaron }\end{array}$ \\
\hline & & & optional & prog & $\begin{array}{l}\text { The C Programming } \\
\text { Language }\end{array}$ & $\begin{array}{l}\text { Kernighan and } \\
\text { Ritchie }\end{array}$ \\
\hline & & & optional & prog & C: A Reference Manual & Harbison and Steele \\
\hline \multirow[t]{8}{*}{$9.5(11 / 8)$} & $\begin{array}{l}\text { University of } \\
\text { Michigan - } \\
\text { Ann Arbor }\end{array}$ & $\begin{array}{l}\text { EECS } 203 \text { Discrete } \\
\text { Mathematics }\end{array}$ & required & math & $\begin{array}{l}\text { Discrete Mathematics } \\
\text { and its Applications }\end{array}$ & Kenneth Rosen \\
\hline & - & $\begin{array}{l}\text { EECS } 280 \\
\text { Programming and } \\
\text { Introductory Data } \\
\text { Structures }\end{array}$ & required & prog & NA & NA \\
\hline & & & optional & prog & $\mathrm{C}++$ Primer & $\begin{array}{l}\text { Lippman, Lajoie and } \\
\text { Moo }\end{array}$ \\
\hline & & $\begin{array}{l}\text { EECS } 281 \text { Data } \\
\text { Structures and } \\
\text { Algorithms }\end{array}$ & required & alg & $\begin{array}{l}\text { Introduction to } \\
\text { Algorithms }\end{array}$ & $\begin{array}{l}\text { Cormen, Leiserson, } \\
\text { Rivest and Stein }\end{array}$ \\
\hline & & & optional & data_struc & $\begin{array}{l}\text { Data Structures and } \\
\text { Their Algorithms }\end{array}$ & $\begin{array}{l}\text { Lewis and } \\
\text { Denenberg }\end{array}$ \\
\hline & & & optional & alg & Algorithms & $\begin{array}{l}\text { Dasgupta, } \\
\text { Papadimitriou, and } \\
\text { Vazirani }\end{array}$ \\
\hline & & $\begin{array}{l}\text { EECS } 370 \text { Introduction } \\
\text { to Computer } \\
\text { Organization }\end{array}$ & required & sys_org & $\begin{array}{l}\text { Computer Organization } \\
\text { and Design: The } \\
\text { Hardware/Software } \\
\text { Interface, ARM Edition }\end{array}$ & $\begin{array}{l}\text { Patterson and } \\
\text { Hennessy }\end{array}$ \\
\hline & & $\begin{array}{l}\text { EECS } 376 \text { Foundations } \\
\text { of Computer Science }\end{array}$ & optional & theory & $\begin{array}{l}\text { Introduction to the } \\
\text { Theory of Computation }\end{array}$ & Michael Sipser \\
\hline
\end{tabular}




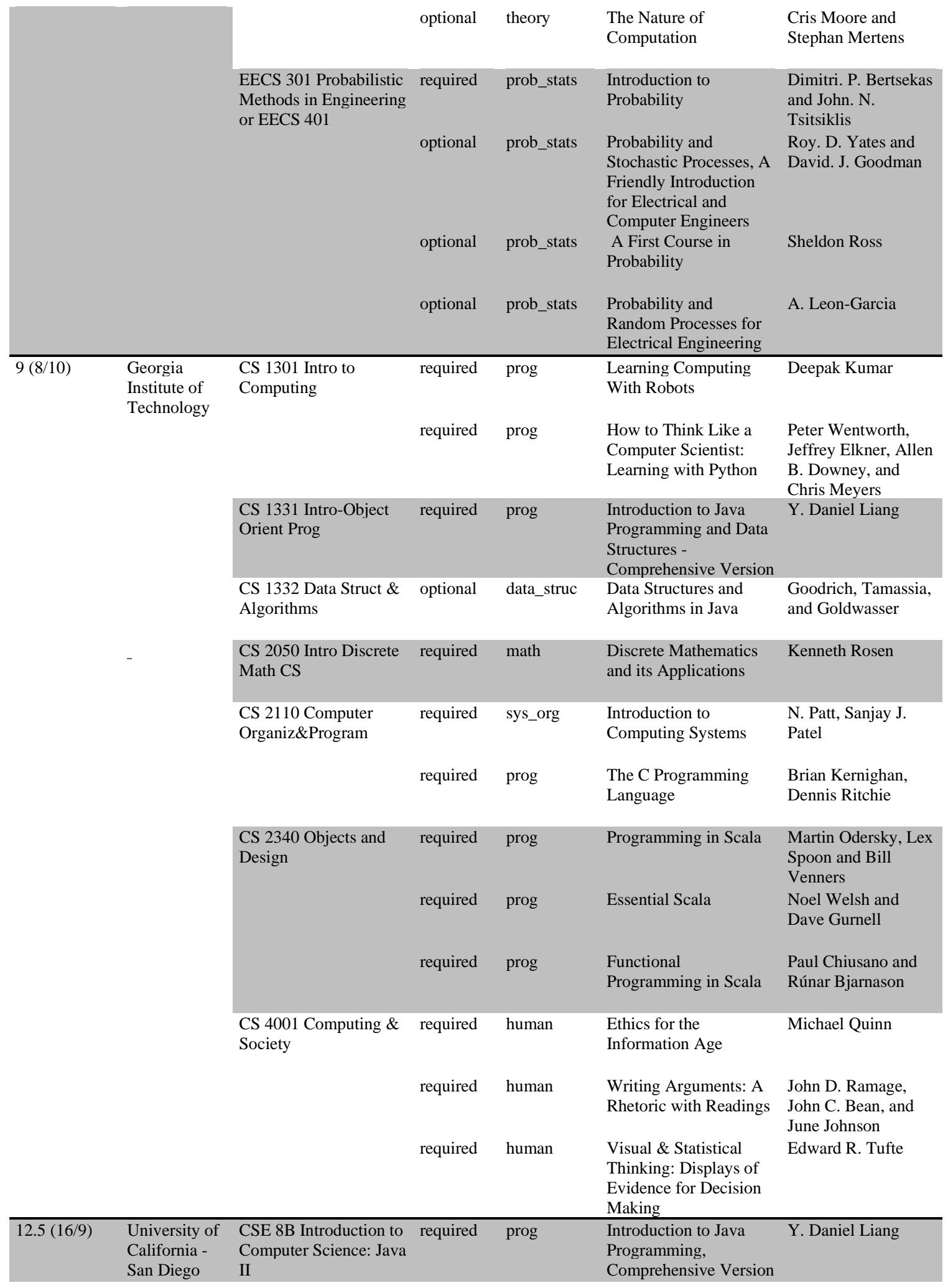




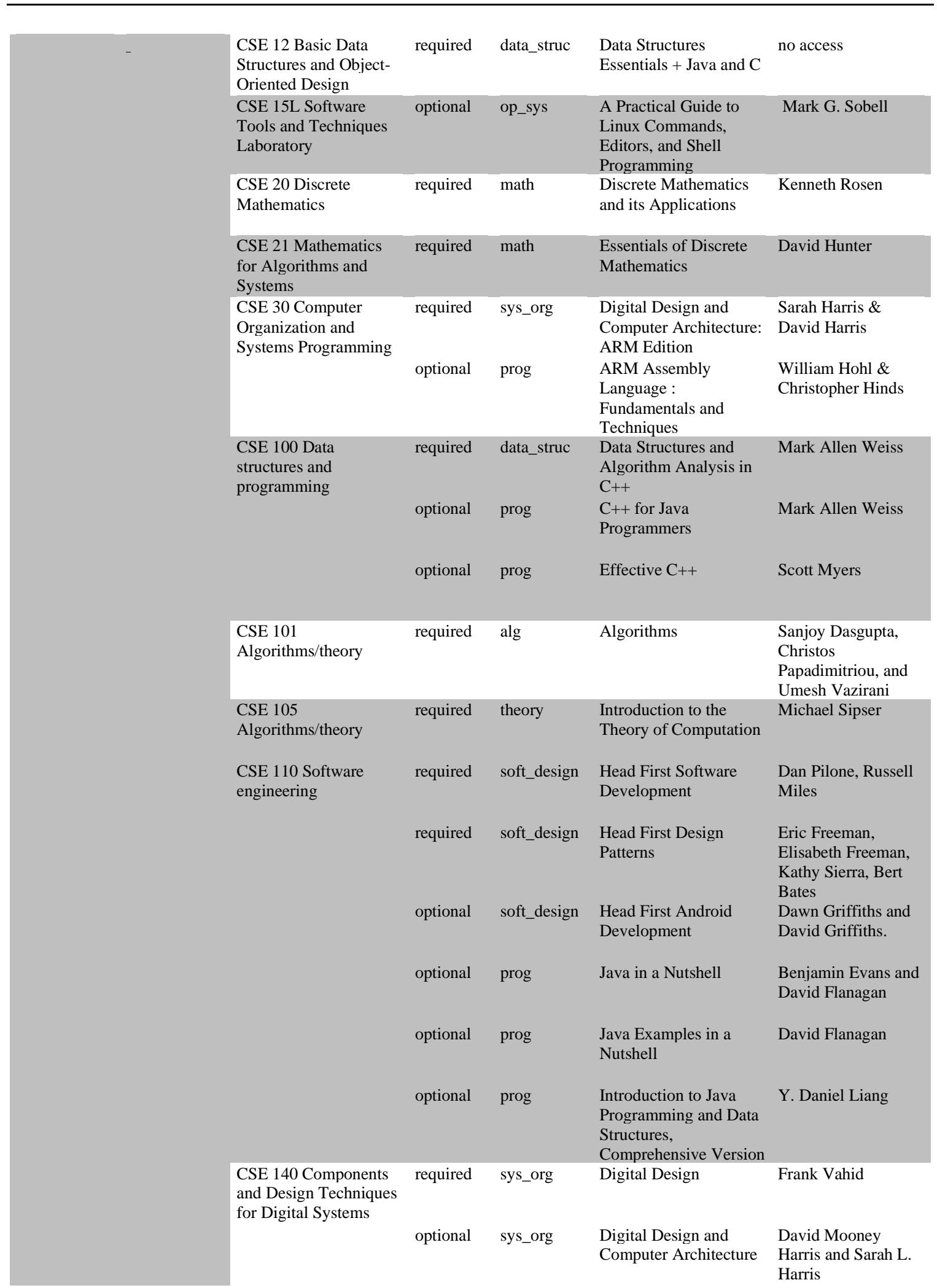




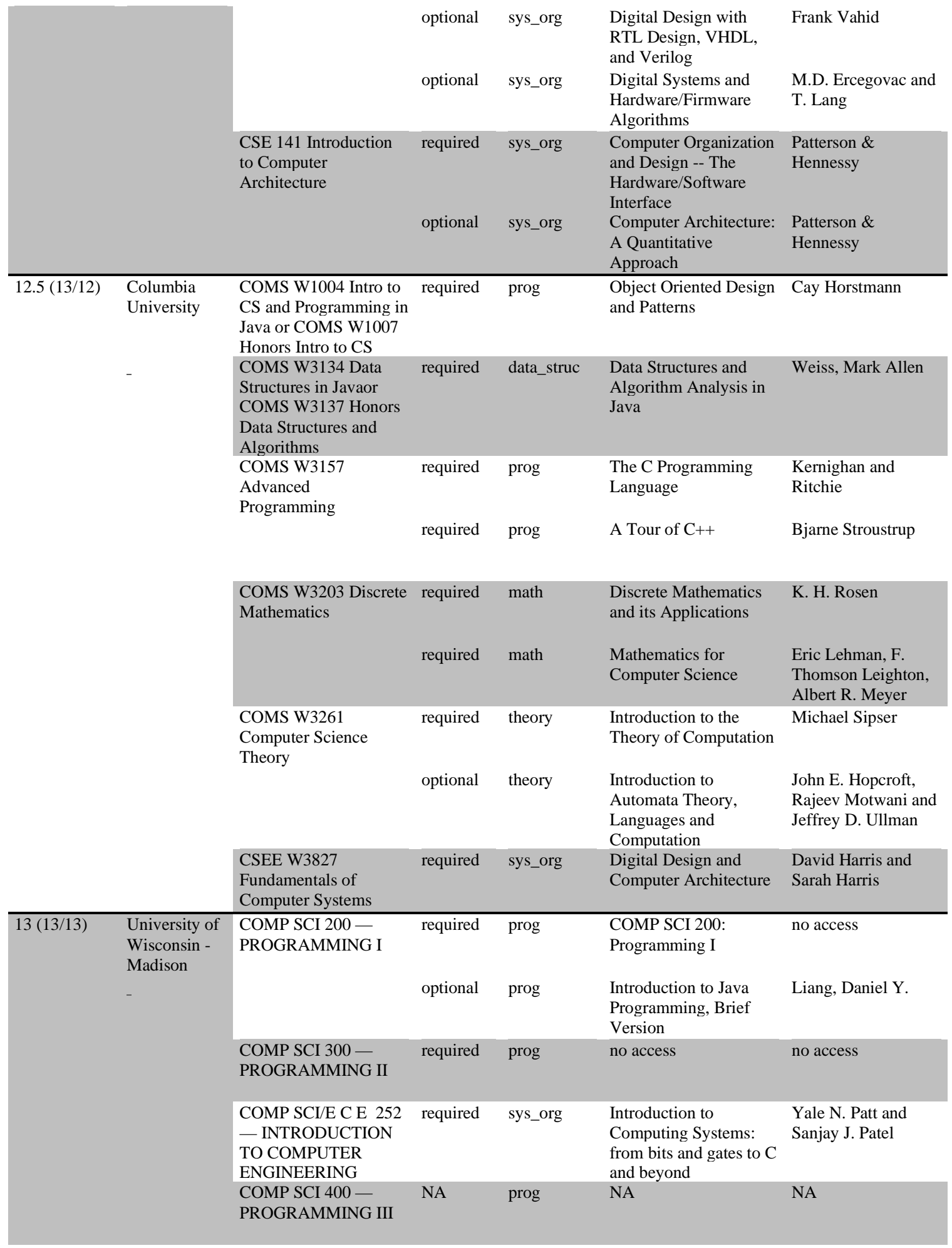




\begin{tabular}{|c|c|c|c|c|c|c|}
\hline & & $\begin{array}{l}\text { COMP SCI/ } \\
\text { MATH } 240- \\
\text { INTRODUCTION TO } \\
\text { DISCRETE } \\
\text { MATHEMATICS }\end{array}$ & required & math & no access & no access \\
\hline & & $\begin{array}{l}\text { COMP SCI/ECE } 354 \\
\text { - MACHINE } \\
\text { ORGANIZATION } \\
\text { AND } \\
\text { PROGRAMMING }\end{array}$ & required & sys_org & $\begin{array}{l}\text { Computer Systems: A } \\
\text { Programmer's } \\
\text { Perspective }\end{array}$ & $\begin{array}{l}\text { Randal E. Bryant, } \\
\text { David R. O'Hallaron }\end{array}$ \\
\hline & & & optional & prog & $\begin{array}{l}\text { C Programming } \\
\text { Language }\end{array}$ & $\begin{array}{l}\text { Brian W. } \\
\text { Kernighan , Dennis } \\
\text { M. Ritchie }\end{array}$ \\
\hline & & $\begin{array}{l}\text { COMP SCI } 577- \\
\text { INTRODUCTION TO } \\
\text { ALGORITHMS }\end{array}$ & required & alg & Algorithms, Etc. & Jeff Erickson \\
\hline & & & optional & alg & Algorithm Design & $\begin{array}{l}\text { by Kleinberg and } \\
\text { Tardos }\end{array}$ \\
\hline \multirow[t]{7}{*}{$13.5(16 / 11)$} & $\begin{array}{l}\text { University of } \\
\text { Maryland - } \\
\text { College Park }\end{array}$ & $\begin{array}{l}\text { CMSC } 131 \text { (4) Object- } \\
\text { Oriented Programming } \\
\text { I }\end{array}$ & required & prog & $\begin{array}{l}\text { Introduction to } \\
\text { Programming in Java -- } \\
\text { an interdisciplinary } \\
\text { approach }\end{array}$ & $\begin{array}{l}\text { Robert Sedgewick } \\
\text { and Kevin Wayne }\end{array}$ \\
\hline & \multirow[t]{6}{*}{-} & $\begin{array}{l}\text { CMSC } 132 \text { (4) Object- } \\
\text { Oriented Programming } \\
\text { II }\end{array}$ & required & data_struc & $\begin{array}{l}\text { Data Structures \& } \\
\text { Abstractions with Java }\end{array}$ & Carrano, Henry \\
\hline & & $\begin{array}{l}\text { CMSC } 216(4) \\
\text { Introduction to } \\
\text { Computer Systems }\end{array}$ & required & prog & C Programming & K.N. King \\
\hline & & & optional & sys_org & $\begin{array}{l}\text { Computer Systems: A } \\
\text { Programmer's } \\
\text { Perspective }\end{array}$ & $\begin{array}{l}\text { R.E. Bryant and D. } \\
\text { R. O'Hallaron }\end{array}$ \\
\hline & & $\begin{array}{l}\text { CMSC } 250 \text { (4) Discrete } \\
\text { Structures }\end{array}$ & optional & math & Discrete Mathematics & Susanna S. Epp \\
\hline & & $\begin{array}{l}\text { CMSC } 330(3) \\
\text { Organization of } \\
\text { Programming } \\
\text { Languages }\end{array}$ & NA & prog & NA & NA \\
\hline & & $\begin{array}{l}\text { CMSC } 351 \text { (3) } \\
\text { Algorithms }\end{array}$ & required & alg & $\begin{array}{l}\text { Introduction to } \\
\text { Algorithms }\end{array}$ & $\begin{array}{l}\text { Thomas Cormen, } \\
\text { Charles Leiserson, } \\
\text { Ron Rivest, and } \\
\text { Clifford Stein } \\
\end{array}$ \\
\hline \multirow[t]{6}{*}{$13.5(10 / 17)$} & $\begin{array}{l}\text { University of } \\
\text { Texas -Austin }\end{array}$ & $\begin{array}{l}\text { C S 311. Discrete } \\
\text { Mathematics for } \\
\text { Computer Science. }\end{array}$ & optional & math & $\begin{array}{l}\text { Discrete Mathematics } \\
\text { and its Applications }\end{array}$ & Kenneth Rosen \\
\hline & \multirow[t]{5}{*}{ - } & $\begin{array}{l}\text { C S 331. Algorithms } \\
\text { and Complexity }\end{array}$ & required & alg & Algorithm Design & $\begin{array}{l}\text { Kleinberg and } \\
\text { Tardos }\end{array}$ \\
\hline & & $\begin{array}{l}\text { C S 312. Introduction to } \\
\text { Programming. }\end{array}$ & required & prog & $\begin{array}{l}\text { Building Java } \\
\text { Programs: A Back to } \\
\text { Basics Approach }\end{array}$ & $\begin{array}{l}\text { Stuart Reges, Marty } \\
\text { Stepp }\end{array}$ \\
\hline & & $\begin{array}{l}\text { C S 314. Data } \\
\text { Structures }\end{array}$ & required & prog & $\begin{array}{l}\text { Building Java } \\
\text { Programs: A Back to } \\
\text { Basics Approach }\end{array}$ & $\begin{array}{l}\text { Stuart Reges , Marty } \\
\text { Stepp }\end{array}$ \\
\hline & & $\begin{array}{l}\text { C S 429. Computer } \\
\text { Organization and } \\
\text { Architecture }\end{array}$ & required & sys_org & $\begin{array}{l}\text { Computer Systems, A } \\
\text { Programmer's } \\
\text { Perspective }\end{array}$ & $\begin{array}{l}\text { Randal E. Bryant } \\
\text { and David } \\
\text { O'Hallaron }\end{array}$ \\
\hline & & $\begin{array}{l}\text { C S 439. Principles of } \\
\text { Computer Systems }\end{array}$ & required & sys_org & $\begin{array}{l}\text { Computer Systems, A } \\
\text { Programmer's } \\
\text { Perspective }\end{array}$ & $\begin{array}{l}\text { Randal E. Bryant } \\
\text { and David } \\
\text { O'Hallaron }\end{array}$ \\
\hline
\end{tabular}




\begin{tabular}{|c|c|c|c|c|c|c|}
\hline & & & required & op_sys & $\begin{array}{l}\text { Operating Systems: } \\
\text { Three Easy Pieces }\end{array}$ & $\begin{array}{l}\text { Remzi H. Arpaci- } \\
\text { Dusseau and Andrea } \\
\text { C. Arpaci-Dusseau }\end{array}$ \\
\hline & & & required & op_sys & $\begin{array}{l}\text { Operating Systems and } \\
\text { Middleware: } \\
\text { Supporting Controlled } \\
\text { Interaction }\end{array}$ & Max Hailperin \\
\hline \multirow[t]{16}{*}{$14(13 / 15)$} & $\begin{array}{l}\text { University of } \\
\text { California - } \\
\text { Los Angeles }\end{array}$ & $\begin{array}{l}\text { COM SCI } 31 \\
\text { Introduction to } \\
\text { Computer Science I }\end{array}$ & required & prog & Absolute $\mathrm{C}++$ & Walter Savitch \\
\hline & - & $\begin{array}{l}\text { COM SCI } 32 \\
\text { Introduction to } \\
\text { Computer Science II }\end{array}$ & required & prog & $\begin{array}{l}\text { Data Abstraction and } \\
\text { Problem Solving with } \\
\text { C++: Walls and Mirrors }\end{array}$ & Frank M. Carrano \\
\hline & & $\begin{array}{l}\text { COM SCI } 33 \\
\text { Introduction to } \\
\text { Computer Organization }\end{array}$ & required & sys_org & $\begin{array}{l}\text { Computer Systems: A } \\
\text { Programmer's } \\
\text { Perspective }\end{array}$ & $\begin{array}{l}\text { Randal E. Bryant } \\
\text { and David R. } \\
\text { O'Hallaron }\end{array}$ \\
\hline & - & $\begin{array}{l}\text { COM SCI 35L } \\
\text { Software Construction } \\
\text { Laboratory }\end{array}$ & NA & soft_design & NA & NA \\
\hline & & $\begin{array}{l}\text { COM SCI M51A Logic } \\
\text { Design of Digital } \\
\text { Systems }\end{array}$ & required & sys_org & $\begin{array}{l}\text { Introduction to Digital } \\
\text { Systems }\end{array}$ & $\begin{array}{l}\text { M.D. Ercegovac, T. } \\
\text { Lang and J. Moreno }\end{array}$ \\
\hline & & & optional & sys_org & $\begin{array}{l}\text { Contemporary Logic } \\
\text { Design }\end{array}$ & R. H. Katz \\
\hline & & $\begin{array}{l}\text { COM SCI } 111 \\
\text { Operating Systems } \\
\text { Principles }\end{array}$ & required & op_sys & $\begin{array}{l}\text { Operating Systems in } \\
\text { Three Easy Pieces }\end{array}$ & $\begin{array}{l}\text { Remzi H. Arpaci- } \\
\text { Dusseau and Andrea } \\
\text { C. Arpaci-Dusseau }\end{array}$ \\
\hline & & & required & sys_org & $\begin{array}{l}\text { Principles of Computer } \\
\text { System Design: An } \\
\text { Introduction }\end{array}$ & $\begin{array}{l}\text { Jerome H. Saltzer } \\
\text { and M. Frans } \\
\text { Kaashoek }\end{array}$ \\
\hline & & $\begin{array}{l}\text { COM SCI } 118 \\
\text { Computer Network } \\
\text { Fundamentals }\end{array}$ & required & & $\begin{array}{l}\text { Computer Networking: } \\
\text { A Top-Down Approach }\end{array}$ & $\begin{array}{l}\text { James F. Kurose and } \\
\text { Keith W. Ross }\end{array}$ \\
\hline & & $\begin{array}{l}\text { COM SCI } 131 \\
\text { Programming } \\
\text { Languages }\end{array}$ & required & prog & $\begin{array}{l}\text { Modern Programming } \\
\text { Languages: A Practical } \\
\text { Introduction }\end{array}$ & $\begin{array}{l}\text { Adam Brooks } \\
\text { Webber }\end{array}$ \\
\hline & & $\begin{array}{l}\text { COM SCI M151B } \\
\text { Computer Systems } \\
\text { Architecture }\end{array}$ & NA & sys_org & NA & NA \\
\hline & & $\begin{array}{l}\text { COM SCI M152A } \\
\text { Introductory Digital } \\
\text { Design Laboratory }\end{array}$ & NA & sys_org & NA & NA \\
\hline & & $\begin{array}{l}\text { COM SCI } 180 \\
\text { Introduction to } \\
\text { Algorithms and } \\
\text { Complexity }\end{array}$ & required & alg & Algorithm Design & $\begin{array}{l}\text { Jon Kleinberg and } \\
\text { Eva Tardos }\end{array}$ \\
\hline & & $\begin{array}{l}\text { COM SCI } 181 \\
\text { Introduction to Formal } \\
\text { Languages and } \\
\text { Automata Theory }\end{array}$ & required & theory & $\begin{array}{l}\text { Introduction to the } \\
\text { Theory of Computation }\end{array}$ & M. Sipser \\
\hline & & $\begin{array}{l}\text { COM SCI } 130 \text { Software } \\
\text { Engineering or } 132 \\
\text { Compiler Construction }\end{array}$ & required & soft_design & Code Complete & Steve McConnell \\
\hline & & & required & soft_design & Software Engineering & Ian Sommerville \\
\hline $14(8 / 20)$ & $\begin{array}{l}\text { Princeton } \\
\text { University }\end{array}$ & $\begin{array}{l}\text { COS } 126 \text { - General } \\
\text { Computer Science }\end{array}$ & required & prog & $\begin{array}{l}\text { Computer Science: An } \\
\text { Interdisciplinary } \\
\text { Approach }\end{array}$ & $\begin{array}{l}\text { R. Sedgewick and K. } \\
\text { Wayne }\end{array}$ \\
\hline
\end{tabular}




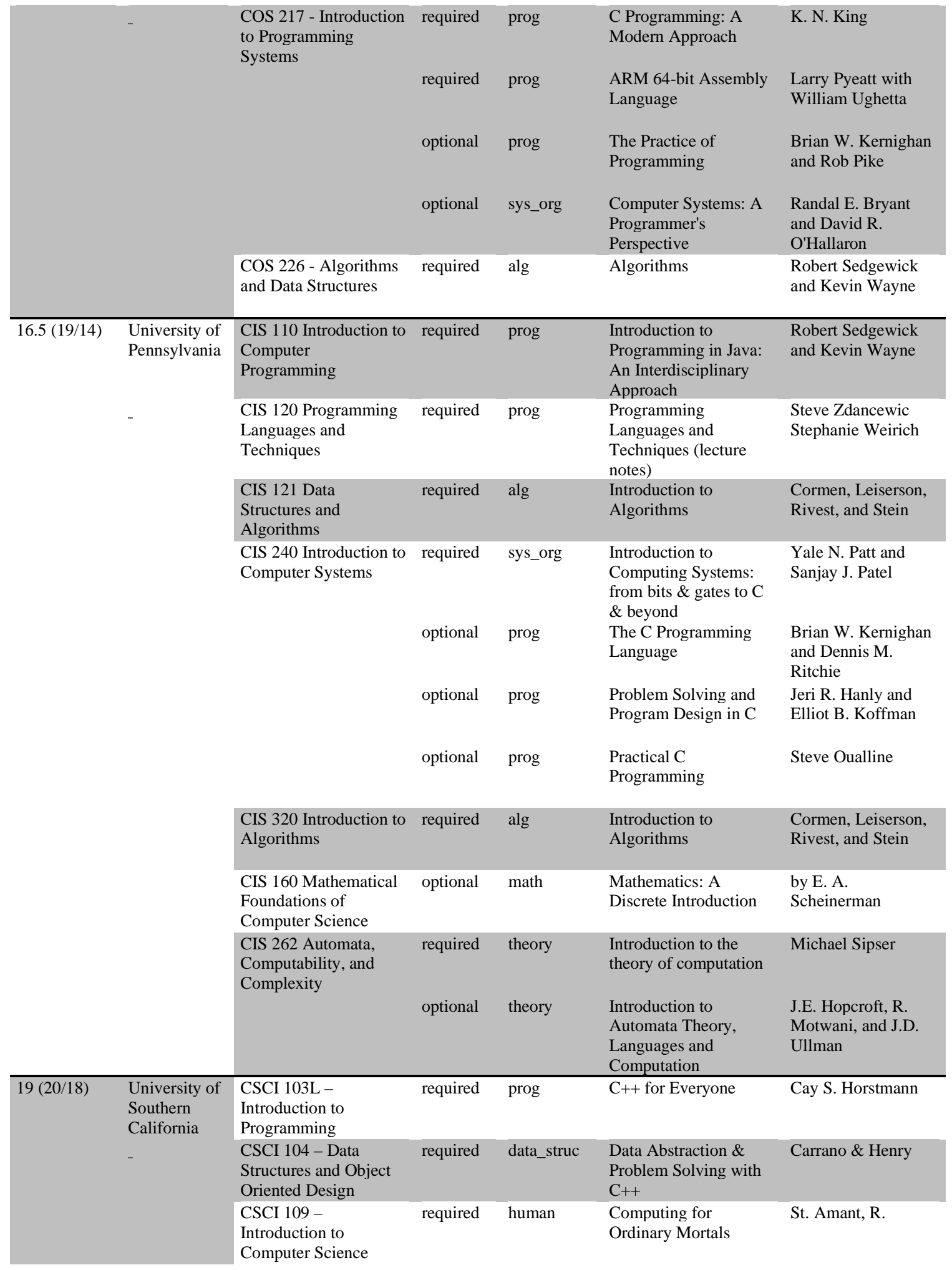




\section{A COMPUTER SCIENCE ACADEMIC VOCABULARY LIST}

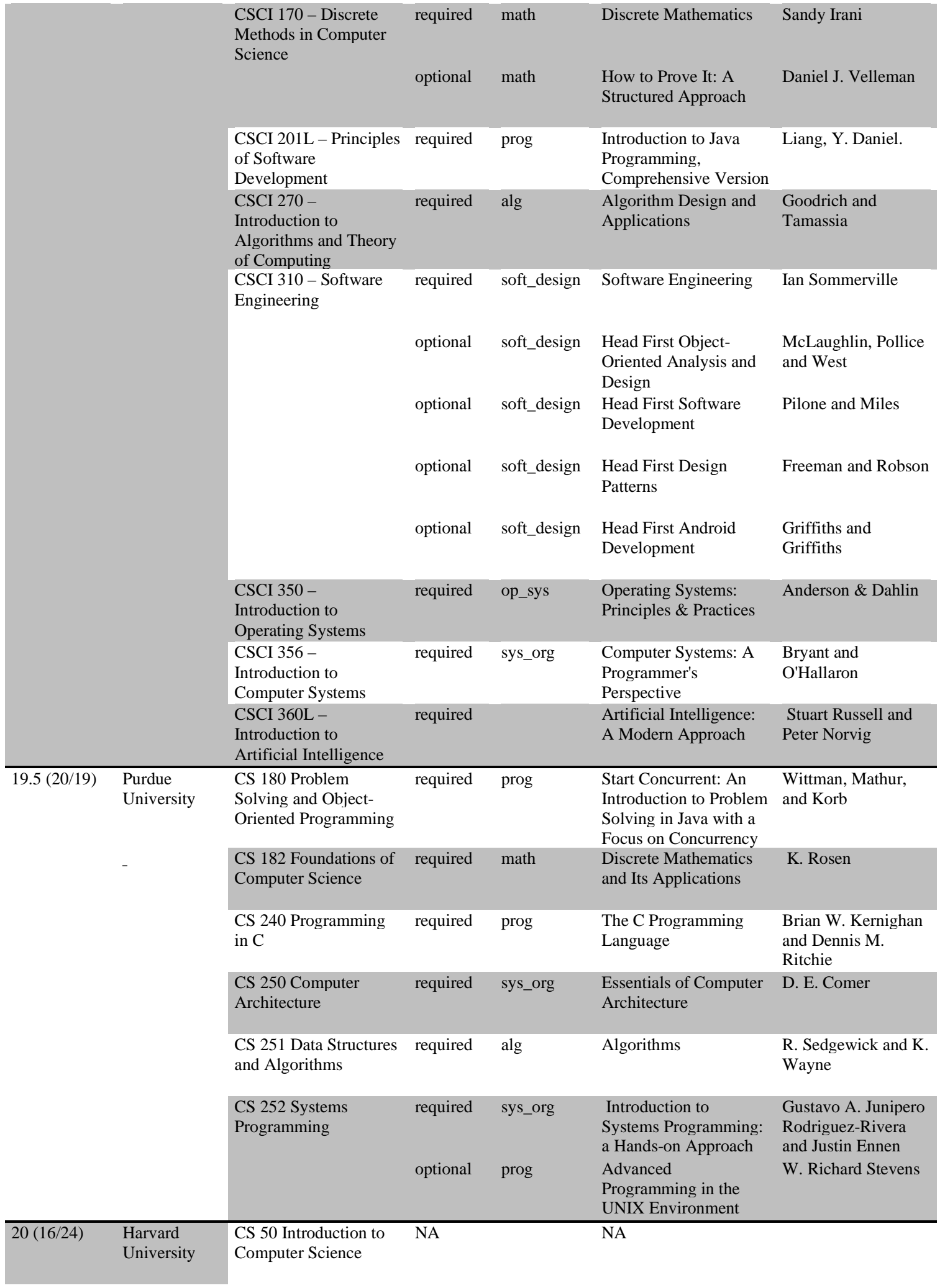




\begin{tabular}{|c|c|c|c|c|c|c|}
\hline & - & $\begin{array}{l}\text { CS } 51 \text { Abstraction and } \\
\text { Design in Computation }\end{array}$ & required & prog & $\begin{array}{l}\text { OCaml from the Very } \\
\text { Beginning }\end{array}$ & John Whitington \\
\hline & & & required & prog & $\begin{array}{l}\text { More OCaml: } \\
\text { Algorithms, Methods \& } \\
\text { Diversions }\end{array}$ & John Whitington \\
\hline & & $\begin{array}{l}\text { CS } 61 \text { Systems } \\
\text { Programming and } \\
\text { Machine Organization }\end{array}$ & required & sys_org & $\begin{array}{l}\text { Computer Systems: A } \\
\text { Programmer's } \\
\text { Perspective }\end{array}$ & $\begin{array}{l}\text { Randal E. Bryant } \\
\text { and David R. } \\
\text { O’Hallaron }\end{array}$ \\
\hline & & $\begin{array}{l}\text { CS } 121 \text { Introduction to } \\
\text { Theoretical Computer } \\
\text { Science }\end{array}$ & required & theory & $\begin{array}{l}\text { Introduction to } \\
\text { Theoretical Computer } \\
\text { Science }\end{array}$ & Boaz Barak \\
\hline & & $\begin{array}{l}\text { CS } 124 \text { Data Structures } \\
\text { and Algorithms }\end{array}$ & optional & alg & $\begin{array}{l}\text { Introduction to } \\
\text { Algorithms }\end{array}$ & $\begin{array}{l}\text { Cormen, Leiserson, } \\
\text { Rivest, and Stein }\end{array}$ \\
\hline & & & optional & alg & Algorithm Design & $\begin{array}{l}\text { Kleinberg and } \\
\text { Tardos }\end{array}$ \\
\hline $\begin{array}{l}110.5 \\
(119 / 102)\end{array}$ & $\begin{array}{l}\text { Portland State } \\
\text { University }\end{array}$ & $\begin{array}{l}\text { CS } 162 \text { Introduction to } \\
\text { Computer Science }\end{array}$ & required & prog & $\begin{array}{l}\text { C++ Programming: } \\
\text { From Problem Analysis } \\
\text { to Program Design }\end{array}$ & D. S. Malik \\
\hline & & & required & prog & $\begin{array}{l}\text { An Introduction to } \\
\text { Computing \& } \\
\text { Algorithms }\end{array}$ & Russell Shackelford \\
\hline & & CS 163 Data Structures & required & data_struc & $\begin{array}{l}\text { Data Abstraction and } \\
\text { Problem Solving with } \\
\text { C++ }\end{array}$ & Frank Carrano \\
\hline & & $\begin{array}{l}\text { CS } 201 \text { Computer } \\
\text { Systems Programming }\end{array}$ & required & sys_org & $\begin{array}{l}\text { Computer Systems: A } \\
\text { Programmer's } \\
\text { Perspective }\end{array}$ & $\begin{array}{l}\text { Bryant and } \\
\text { O'Hallaron }\end{array}$ \\
\hline & & & required & prog & $\begin{array}{l}\text { The C Programming } \\
\text { Language }\end{array}$ & $\begin{array}{l}\text { Kernighan and } \\
\text { Ritchie }\end{array}$ \\
\hline & & $\begin{array}{l}\text { CS } 202 \text { Programming } \\
\text { Systems }\end{array}$ & required & prog & $\mathrm{C}++$ Primer Plus & Stephen Prata \\
\hline & & & required & prog & Thinking in Java & Bruce Eckel \\
\hline & & $\begin{array}{l}\text { CS } 250 \text { Discrete } \\
\text { Structures I }\end{array}$ & required & math & $\begin{array}{l}\text { Notes on Discrete } \\
\text { Mathematics }\end{array}$ & Miguel Lerma \\
\hline & & $\begin{array}{l}\text { CS } 251 \text { Discrete } \\
\text { Structures II }\end{array}$ & required & math & $\begin{array}{l}\text { Discrete Structures, } \\
\text { Logic, and } \\
\text { Computability }\end{array}$ & James Hein \\
\hline & & $\begin{array}{l}\text { CS } 300 \text { Elements of } \\
\text { Software Engineering }\end{array}$ & required & soft_design & $\begin{array}{l}\text { Engineering Software } \\
\text { Products }\end{array}$ & Ian Sommerville \\
\hline & & $\begin{array}{l}\text { CS } 305 \text { Social, Ethical, } \\
\text { and Legal Issues }\end{array}$ & required & human & $\begin{array}{l}\text { Ethics for the } \\
\text { Information Age }\end{array}$ & Michael Quinn \\
\hline & & $\begin{array}{l}\text { CS } 320 \text { Principles of } \\
\text { Programming } \\
\text { Languages }\end{array}$ & NA & prog & NA & \\
\hline & & $\begin{array}{l}\text { CS } 333 \text { Intro to } \\
\text { Operating Systems }\end{array}$ & required & op_sys & $\begin{array}{l}\text { Operating Systems: } \\
\text { Three Easy Pieces }\end{array}$ & $\begin{array}{l}\text { Remzi H. Arpaci- } \\
\text { Dusseau and Andrea } \\
\text { C. Arpaci-Dusseau }\end{array}$ \\
\hline & & $\begin{array}{l}\text { CS } 350 \text { Algorithms and } \\
\text { Complexity }\end{array}$ & required & alg & $\begin{array}{l}\text { Introduction to the } \\
\text { Design and Analysis of } \\
\text { Algorithms }\end{array}$ & Anany Levitin \\
\hline
\end{tabular}




\section{A COMPUTER SCIENCE ACADEMIC VOCABULARY LIST}

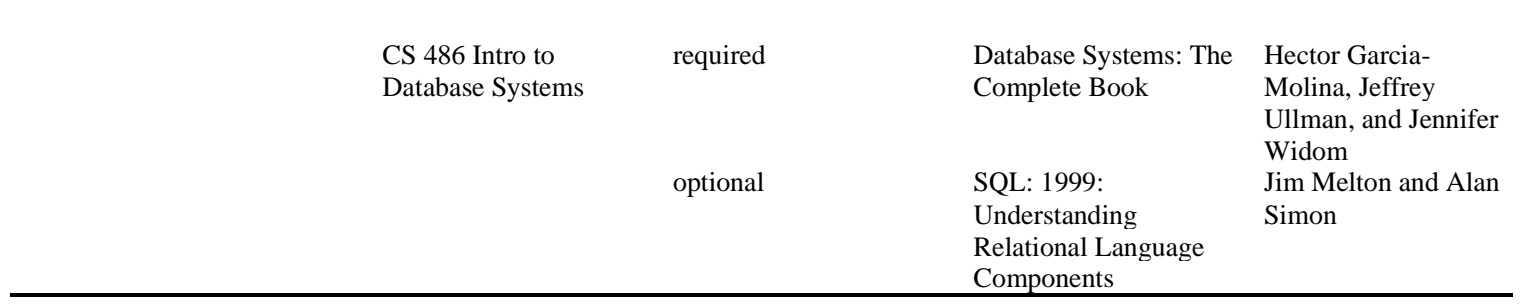




\section{Appendix D: $\quad$ Textbook Usage Counts}

Table D.2 core CS textbook usage counts

\begin{tabular}{|c|c|c|c|}
\hline $\begin{array}{l}\text { Text } \\
\text { category } \\
\end{array}$ & Title & Authors & $\begin{array}{l}\text { Usage } \\
\text { Frequency }\end{array}$ \\
\hline alg & Introduction to Algorithms & $\begin{array}{l}\text { Cormen, Leiserson, } \\
\text { Rivest, and Stein }\end{array}$ & 9 \\
\hline alg & Algorithm Design & Kleinberg \& Tardos & 6 \\
\hline alg & Algorithms & $\begin{array}{l}\text { Dasgupta, Papadimitriou, } \\
\text { and Vazirani }\end{array}$ & 4 \\
\hline alg & Algorithms & Sedgewick \& Wayne & 3 \\
\hline data_struc & $\begin{array}{l}\text { Data Structures and Algorithm } \\
\text { Analysis in Java }\end{array}$ & Weiss & 2 \\
\hline data_struc & $\begin{array}{l}\text { Data Abstraction \& Problem } \\
\text { Solving with C++ }\end{array}$ & Carrano \& Henry & 2 \\
\hline human & Ethics for the Information Age & Quinn & 2 \\
\hline human & $\begin{array}{l}\text { Ethics in Information } \\
\text { Technology }\end{array}$ & Reynolds & 1 \\
\hline human & Coders & Thompson & 1 \\
\hline math & $\begin{array}{l}\text { Discrete Mathematics and its } \\
\text { Applications }\end{array}$ & Rosen & 6 \\
\hline math & $\begin{array}{l}\text { Mathematics for Computer } \\
\text { Science }\end{array}$ & $\begin{array}{l}\text { Lehman, Leighton, and } \\
\text { Meyer }\end{array}$ & 3 \\
\hline op_sys & $\begin{array}{l}\text { Operating Systems in Three } \\
\text { Easy Pieces }\end{array}$ & $\begin{array}{l}\text { Arpaci-Dusseau \& } \\
\text { Arpaci-Dusseau }\end{array}$ & 4 \\
\hline op_sys & $\begin{array}{l}\text { Operating Systems: Principles } \\
\text { and Practice }\end{array}$ & Anderson \& Dahlin & 2 \\
\hline prob_stats & Introduction to Probability & Bertsekas \& Tsitsiklis & 2 \\
\hline prob_stats & $\begin{array}{l}\text { Probability and Statistics for } \\
\text { Computer Science }\end{array}$ & Forsyth & 1 \\
\hline prog & The C Programming Language & Kernighan \& Ritchie & 10 \\
\hline prog & $\begin{array}{l}\text { Introduction to Java } \\
\text { Programming and Data } \\
\text { Structures, Comprehensive } \\
\text { Version }\end{array}$ & Liang & 5 \\
\hline prog & $\begin{array}{l}\text { Building Java Programs: A } \\
\text { Back to Basics Approach }\end{array}$ & Reges \& Stepp & 4 \\
\hline prog & $\begin{array}{l}\text { Introduction to Programming in } \\
\text { Java: An Interdisciplinary } \\
\text { Approach }\end{array}$ & Sedgewick \& Wayne & 3 \\
\hline soft_design & Software Engineering & Sommerville & 2 \\
\hline
\end{tabular}




\begin{tabular}{|c|c|c|c|}
\hline soft_design & $\begin{array}{l}\text { Head First Software } \\
\text { Development }\end{array}$ & Pilone \& Miles & 2 \\
\hline sys_org & $\begin{array}{l}\text { Computer Systems: A } \\
\text { Programmer's Perspective }\end{array}$ & Bryant \& O'Hallaron & 13 \\
\hline sys_org & $\begin{array}{l}\text { Computer Organization and } \\
\text { Design: The Hardware Software } \\
\text { Interface (RISC-V Edition). }\end{array}$ & Patterson \& Hennessy & 6 \\
\hline sys_org & $\begin{array}{l}\text { Introduction to Computing } \\
\text { Systems: from bits and gates to } \\
\text { C and beyond }\end{array}$ & Patt \& Patel & 3 \\
\hline sys_org & $\begin{array}{l}\text { Principles of Computer System } \\
\text { Design: An Introduction }\end{array}$ & Saltzer \& Kaashoek & 4 \\
\hline sys_org & $\begin{array}{l}\text { Digital Design and Computer } \\
\text { Architecture }\end{array}$ & Harris \& Harris & 3 \\
\hline theory & $\begin{array}{l}\text { Introduction to the Theory of } \\
\text { Computation }\end{array}$ & Sipser & 8 \\
\hline theory & The Nature of Computation & Moore \& Mertens & 3 \\
\hline theory & $\begin{array}{l}\text { Introduction to Theoretical } \\
\text { Computer Science }\end{array}$ & Barak & 2 \\
\hline theory & $\begin{array}{l}\text { Introduction to Automata } \\
\text { Theory, Languages and } \\
\text { Computation }\end{array}$ & $\begin{array}{l}\text { Hopcroft, Motwani and } \\
\text { Ullman }\end{array}$ & 2 \\
\hline
\end{tabular}




\section{Appendix E: $\quad$ CSAC1 \& CSAC2 Token counts}

Table E.3 CSAC1 Computer system organization ACM article sub-corpus

\begin{tabular}{llr}
\hline Article Category & CSAC1 (175k target) & Tokens \\
\hline $\begin{array}{l}\text { Computer system } \\
\text { organization }\end{array}$ & (Alsaedi et al., 2017) & 9,404 \\
& (Ardagna et al., 2015) & 13,265 \\
& (Bleiholder \& Naumann, 2008) & 16,968 \\
& (Bulling et al., 2014) & 11,909 \\
& (Davis \& Burns, 2011) & 14,201 \\
& (Deng, 2011) & 12,745 \\
& (Dietrich \& Dressler, 2009) & 14,223 \\
& (Fung et al., 2009) & 13,969 \\
& (Hoffman et al., 2009) & 13,297 \\
& (Mastelic et al., 2014) & 13,294 \\
& (Shuja et al., 2016) & 14,164 \\
& (Suomela, 2013) & 15,810 \\
& (Wilson et al., 2012) & 12,922 \\
\cline { 2 - 3 } & \multicolumn{2}{c}{ Total token count: } \\
Mean token count: & 176,171 \\
&
\end{tabular}

Table E.4 CSAC1 Computing methodologies ACM article sub-corpus

\begin{tabular}{llr}
\hline Article Category & CSAC1 (175k target) & Tokens \\
\hline Computing & (Aggarwal \& Ryoo, 2011) & 18,792 \\
methodologies & (Barnes et al., 2009) & 8,584 \\
& (Corbett et al., 2013) & 9,595 \\
& (Davis et al., 2014) & 7,304 \\
& (Djeu et al., 2011) & 20,024 \\
& (Gama et al., 2014) & 15,656 \\
& (Hasinoff et al., 2016) & 9,530 \\
& (Horvath \& Geiger, 2009) & 6,534 \\
& (Papalexakis et al., 2016) & 16,798 \\
& (Pouyanfar et al., 2018) & 14,657 \\
& (Qi \& Davison, 2009) & 10,890 \\
& (Reddy et al., 2010) & 9,600 \\
& (Rodríguez et al., 2014) & 12,389 \\
& (Silva et al., 2013) & 12,332 \\
& (Wang \& Popović, 2009) & 5,306 \\
\cline { 2 - 3 } & \multicolumn{2}{c}{ Total token count: } \\
& \multicolumn{2}{c}{ Mean token count: } \\
\end{tabular}


Table E.5 CSAC1 Hardware ACM article sub-corpus

\begin{tabular}{llr}
\hline Article Category & CSAC1 (175k target) & Tokens \\
\hline Hardware & (Aaraj et al., 2008) & 8,940 \\
& (Alaghi \& Hayes, 2013) & 6,374 \\
& (Apalkov et al., 2013) & 14,134 \\
& (Calabrese et al., 2014) & 8,011 \\
& (Henson \& Taylor, 2014) & 11,129 \\
& (Lanman \& Luebke, 2013) & 7,117 \\
& (Lien et al., 2016) & 12,651 \\
& (Maimone et al., 2017) & 11,983 \\
& (Medina \& García, 2014) & 16,141 \\
& (Ni et al., 2009) & 12,091 \\
& (Sen et al., 2013) & 16,166 \\
& (Shin et al., 2010) & 11,991 \\
& (Vasilomanolakis et al., 2015) & 15,019 \\
& (Woodcock et al., 2009) & 13,266 \\
& (Yang et al., 2013) & 11,944 \\
\cline { 2 - 2 } Total token count: & 176,957 \\
& \multicolumn{2}{c}{ Mean token count: } \\
\hline
\end{tabular}

Table E.6 CSAC1 Human centered computing ACM article sub-corpus

\begin{tabular}{llr}
\hline Article Category & CSAC1 (175k target) & Tokens \\
\hline Human centered & (Bau \& Poupyrev, 2012) & 8,015 \\
computing & (Cao et al., 2013) & 8,493 \\
& (Carminati et al., 2009) & 15,636 \\
& (Coppola \& Morisio, 2016) & 13,748 \\
& (Coros et al., 2013) & 9,380 \\
& (Feng et al., 2010) & 15,011 \\
& (Gerber \& Hui, 2013) & 14,341 \\
& (Hassenzahl et al., 2012) & 7,849 \\
& (Höök \& Löwgren, 2012) & 8,467 \\
& (Iriberri \& Leroy, 2009) & 10,801 \\
& (Lindgaard et al., 2011) & 11,788 \\
& (Medhi et al., 2011) & 11,459 \\
& (Reinecke \& Bernstein, 2011) & 11,632 \\
& (Rosenberg \& Perlin, 2009) & 6,389 \\
& (Wang \& Mark, 2015) & 9,842 \\
& (Weerasiri et al., 2017) & 13,326 \\
\cline { 2 - 3 } & \multicolumn{2}{c}{ Total token count: } \\
& Mean token count: & 176,177 \\
\end{tabular}


Table E.7 CSAC1 Information systems ACM article sub-corpus

\begin{tabular}{llr}
\hline Article Category & CSAC1 (175k target) & Tokens \\
\hline Information systems & (Batini et al., 2009) & 19,692 \\
& (Beatty et al., 2011) & 17,110 \\
& (Bonchi et al., 2011) & 16,580 \\
& (Cacheda et al., 2011) & 12,781 \\
& (Carpineto \& Romano, 2012) & 19,299 \\
& (Carpineto et al., 2009) & 16,412 \\
& (Mooney \& Roddick, 2013) & 14,332 \\
& (Parent et al., 2013) & 14,322 \\
& (Shaul \& Tauber, 2013) & 10,776 \\
& (Shi et al., 2014) & 17,313 \\
& (Zheng, 2015) & 16,613 \\
\cline { 2 - 2 } & \multicolumn{2}{c}{ Total token count: } \\
Mean token count: & 175,230 \\
&
\end{tabular}

Table E.8 CSAC1 Mathematics of computing ACM article sub-corpus

\begin{tabular}{|c|c|c|}
\hline Article Category & CSAC1 (175k target) & Tokens \\
\hline \multirow{19}{*}{$\begin{array}{l}\text { Mathematics of } \\
\text { computing }\end{array}$} & (Blei et al., 2010) & 10,740 \\
\hline & (Candès et al., 2011) & 13,715 \\
\hline & (Chentanez \& Müller, 2011) & 6,818 \\
\hline & (Chentanez et al., 2009) & 8,162 \\
\hline & (Esling \& Agon, 2012) & 13,109 \\
\hline & (Kim \& Pollard, 2011) & 15,109 \\
\hline & (Martínez et al., 2016) & 12,393 \\
\hline & (Moser \& Tardos, 2010) & 6,964 \\
\hline & (Nielsen et al., 2015) & 17,088 \\
\hline & (Patterson \& Rao, 2014) & 8,390 \\
\hline & (Rao et al., 2010) & 9,244 \\
\hline & (Schumacher et al., 2015) & 9,443 \\
\hline & $(\mathrm{Si}, 2015)$ & 14,495 \\
\hline & (Solenthaler \& Gross, 2011) & 4,942 \\
\hline & (Stava et al., 2012) & 9,169 \\
\hline & (Weber et al., 2009) & 8,506 \\
\hline & (Zhou et al., 2013) & 6,915 \\
\hline & Total token count: & 175,202 \\
\hline & Mean token count: & 10,306 \\
\hline
\end{tabular}


Table E.9 CSAC1 Networks ACM article sub-corpus

\begin{tabular}{llr}
\hline Article Category & CSAC1 (175k target) & Tokens \\
\hline Networks & (Baccour et al., 2012) & 13,269 \\
& (Di Francesco et al., 2011) & 13,332 \\
& (Eisenman et al., 2009) & 15,392 \\
& (Gaeta, 2018) & 8,191 \\
& (Huang et al., 2018) & 15,245 \\
& (Jordan, 2009) & 12,246 \\
(Kurian \& Sarac, 2010) & 13,404 \\
& (Law et al., 2009) & 12,828 \\
& (Li \& Liu, 2009) & 10,186 \\
& (Mitchell \& Chen, 2014) & 11,335 \\
& (Muir \& Oorschot, 2009) & 11,168 \\
& (Razzaque et al., 2013) & 18,665 \\
& (Walfish et al., 2010) & 19,810 \\
\cline { 2 - 2 } Total token count: & 175,071 \\
Mean token count: & 13,467 \\
\hline
\end{tabular}

Table E.10 CSAC1 Security and privacy ACM article sub-corpus

\begin{tabular}{llr}
\hline Article Category & CSAC1 (175k target) & \multicolumn{1}{c}{ Tokens } \\
\hline Security and privacy & (Abadi et al., 2009) & 14,549 \\
& (Acquisti et al., 2017) & 18,162 \\
& (Biddle et al., 2012) & 18,054 \\
& (Chang et al., 2013) & 12,938 \\
& (Diesburg \& Wang, 2010) & 14,382 \\
& (Enck et al., 2014) & 11,455 \\
& (Fung et al., 2010) & 12,026 \\
& (Heartfield \& Loukas, 2015) & 15,582 \\
& (Liu et al., 2011) & 14,384 \\
& (Rodríguez-Gómez et al., 2013) & 13,688 \\
& (Roemer et al., 2012) & 13,645 \\
& (Ye et al., 2017) & 16,388 \\
\cline { 2 - 2 } & \multicolumn{2}{c}{ Total token count: } \\
Mean token count: & 175,253 \\
& \multicolumn{2}{c}{14,604} \\
\hline
\end{tabular}


Table E.11 CSAC1 Software and its engineering ACM article sub-corpus

\begin{tabular}{llr}
\hline Article Category & CSAC1 (175k target) & \multicolumn{1}{c}{ Tokens } \\
\hline Software and its & (Cockburn et al., 2008) & 11,347 \\
engineering & (Harman et al., 2012) & 13,200 \\
& (Ko et al., 2011) & 17,007 \\
& (Largent, 2016) & 9,298 \\
& (Lopes et al., 2017) & 8,770 \\
& (Mendling et al., 2018) & 6,002 \\
& (Mili et al., 2010) & 20,652 \\
& (Mottola \& Picco, 2011) & 18,056 \\
& (Ouyang et al., 2009) & 12,955 \\
& (Shahriar \& Zulkernine, 2012) & 20,291 \\
& (Stefik \& Siebert, 2013) & 18,035 \\
& (Toosi et al., 2014) & 20,110 \\
\cline { 2 - 3 } & \multicolumn{2}{c}{ Total token count: } \\
& \multicolumn{2}{c}{ Mean token count: } \\
\end{tabular}

Table E.12 CSAC1 Theory of computation ACM article sub-corpus

\begin{tabular}{|c|c|c|}
\hline Article Category & CSAC1 (175k target) & Tokens \\
\hline \multirow{20}{*}{$\begin{array}{l}\text { Theory of } \\
\text { computation }\end{array}$} & (Arora et al., 2009) & 15,767 \\
\hline & (Bermano et al., 2011) & 6,563 \\
\hline & (Bokeloh et al., 2010) & 8,321 \\
\hline & (Bommes et al., 2009) & 7,039 \\
\hline & (Calì et al., 2012) & 5,428 \\
\hline & (Cederman \& Tsigas, 2009) & 7,134 \\
\hline & (Farbman et al., 2009) & 6,135 \\
\hline & (Gal et al., 2009) & 6,657 \\
\hline & (Gao et al., 2010) & 24,224 \\
\hline & (Jung et al., 2017) & 18,000 \\
\hline & (Martin et al., 2010) & 6,856 \\
\hline & (Palubicki et al., 2009) & 5,937 \\
\hline & (Shankar, 2009) & 21,965 \\
\hline & (Shao et al., 2012) & 7,695 \\
\hline & (Tagliasacchi et al., 2009) & 6,177 \\
\hline & (Wang et al., 2013) & 6,890 \\
\hline & (Wei \& Chai, 2010) & 6,841 \\
\hline & (Zhu et al., 2012) & 7,530 \\
\hline & Total token count: & 175,159 \\
\hline & Mean token count: & 9,731 \\
\hline
\end{tabular}


Table E.13 CSAC1 Algorithms textbook sub-corpus

\begin{tabular}{llr}
\hline Textbook Category & CSAC1 (175k target) & \multicolumn{1}{c}{ Tokens } \\
\hline Algorithms & (Cormen et al., 2009) Ch. 01 & 3,885 \\
& (Cormen et al., 2009) Ch. 02 & 9,753 \\
& (Cormen et al., 2009) Ch. 03 & 6,163 \\
& (Cormen et al., 2009) Ch. 04 & 14,484 \\
& (Cormen et al., 2009) Ch. 05 & 9,937 \\
& (Cormen et al., 2009) Ch. 06 & 6,647 \\
& (Cormen et al., 2009) Ch. 07 & 6,271 \\
& (Cormen et al., 2009) Ch. 09 & 4,439 \\
& (Cormen et al., 2009) Ch. 10 & 7,319 \\
& (Cormen et al., 2009) Ch. 11 & 11,006 \\
& (Cormen et al., 2009) Ch. 12 & 6,639 \\
& (Cormen et al., 2009) Ch. 13 & 10,088 \\
& (Cormen et al., 2009) Ch. 14 & 6,309 \\
& (Cormen et al., 2009) Ch. 15 & 21,039 \\
\hline & (Cormen et al., 2009) Ch. 16 & 13,879 \\
& (Cormen et al., 2009) Ch. 17 & 10,100 \\
& (Cormen et al., 2009) Ch. 18 & 8,090 \\
& (Cormen et al., 2009) Ch. 19 & 8,209 \\
& (Cormen et al., 2009) Ch. 20 & 10,552 \\
\hline & Total token count: & 174,809 \\
Mean token count: & 9,200 \\
\hline
\end{tabular}

Table E.14 CSAC1 Data structures textbook sub-corpus

\begin{tabular}{llr}
\hline Textbook Category & CSAC1 (175k target) & \multicolumn{2}{r}{ Tokens } \\
\hline Data structures & (Weiss, 2011) Ch. 01 & 8,542 \\
& (Weiss, 2011) Ch. 02 & 9,791 \\
& (Weiss, 2011) Ch. 03 & 14,258 \\
& (Weiss, 2011) Ch. 04 & 19,372 \\
& (Weiss, 2011) Ch. 05 & 16,544 \\
& (Weiss, 2011) Ch. 06 & 12,249 \\
& (Weiss, 2011) Ch. 07 & 19,456 \\
& (Weiss, 2011) Ch. 08 & 8,293 \\
& (Weiss, 2011) Ch. 09 & 20,904 \\
& (Weiss, 2011) Ch. 10 & 25,860 \\
& (Weiss, 2011) Ch. 11 & 8,836 \\
& (Weiss, 2011) Ch. 12 & 15,689 \\
\hline \multicolumn{2}{c}{ Total token count: } \\
Mean token count: & 179,794 \\
& & 14,983 \\
\hline
\end{tabular}


Table E.15 CSAC1 Human centered computing textbook sub-corpus

\begin{tabular}{|c|c|c|}
\hline Textbook Category & CSAC1 (175k target) & Tokens \\
\hline \multirow{11}{*}{$\begin{array}{l}\text { Human centered } \\
\text { computing }\end{array}$} & (Quinn, 2017) Ch. 01 & 17,057 \\
\hline & (Quinn, 2017) Ch. 02 & 26,185 \\
\hline & (Quinn, 2017) Ch. 03 & 20,869 \\
\hline & (Quinn, 2017) Ch. 04 & 25,470 \\
\hline & (Quinn, 2017) Ch. 05 & 17,204 \\
\hline & (Quinn, 2017) Ch. 06 & 18,667 \\
\hline & (Quinn, 2017) Ch. 07 & 15,614 \\
\hline & (Quinn, 2017) Ch. 08 & 19,560 \\
\hline & (Quinn, 2017) Ch. 09 & 16,690 \\
\hline & Total token count: & 177,316 \\
\hline & Mean token count: & 19,702 \\
\hline
\end{tabular}

Table E.16 CSAC1 Mathematics of computing textbook sub-corpus

\begin{tabular}{|c|c|c|}
\hline Textbook Category & CSAC1 (175k target) & Tokens \\
\hline \multirow{7}{*}{$\begin{array}{l}\text { Mathematics of } \\
\text { computing }\end{array}$} & (Rosen, 2012) Ch. 01 & 66,389 \\
\hline & (Rosen, 2012) Ch. 02 & 34,901 \\
\hline & (Rosen, 2012) Ch. 03 & 28,166 \\
\hline & (Rosen, 2012) Ch. 04 & 36,737 \\
\hline & (Rosen, 2012) Ch. 05.1 & 11,652 \\
\hline & Total token count: & 177,845 \\
\hline & Mean token count: & 35,569 \\
\hline
\end{tabular}


Table E.17 CSAC1 Operating systems textbook sub-corpus

\begin{tabular}{|c|c|c|}
\hline $\begin{array}{l}\text { Textbook } \\
\text { Category }\end{array}$ & CSAC1 (175k target) & Tokens \\
\hline \multirow[t]{12}{*}{ Operating systems } & (Arpaci-Dusseau \& Arpaci-Dusseau, 2018) Pt. 01 & 85,580 \\
\hline & (Arpaci-Dusseau \& Arpaci-Dusseau, 2018) Pt. 02 & 42,103 \\
\hline & $\begin{array}{l}\text { (Arpaci-Dusseau \& Arpaci-Dusseau, 2018) Pt. } \\
\text { 03_Ch.35 }\end{array}$ & 332 \\
\hline & $\begin{array}{l}\text { (Arpaci-Dusseau \& Arpaci-Dusseau, 2018) Pt. } \\
\text { 03_Ch.36 }\end{array}$ & 4,388 \\
\hline & $\begin{array}{l}\text { (Arpaci-Dusseau \& Arpaci-Dusseau, 2018) Pt. } \\
\text { 03_Ch.37 }\end{array}$ & 5,383 \\
\hline & $\begin{array}{l}\text { (Arpaci-Dusseau \& Arpaci-Dusseau, 2018) Pt. } \\
\text { 03_Ch.38 }\end{array}$ & 7,065 \\
\hline & $\begin{array}{l}\text { (Arpaci-Dusseau \& Arpaci-Dusseau, 2018) Pt. } \\
\text { 03_Ch.39 }\end{array}$ & 9,943 \\
\hline & $\begin{array}{l}\text { (Arpaci-Dusseau \& Arpaci-Dusseau, 2018) Pt. } \\
\text { 03_Ch.40 }\end{array}$ & 6,983 \\
\hline & $\begin{array}{l}\text { (Arpaci-Dusseau \& Arpaci-Dusseau, 2018) Pt. } \\
\text { 03_Ch.41 }\end{array}$ & 5,089 \\
\hline & $\begin{array}{l}\text { (Arpaci-Dusseau \& Arpaci-Dusseau, 2018) Pt. } \\
\text { 03_Ch.42 }\end{array}$ & 7,983 \\
\hline & & $\begin{array}{r}\text { (47, } 166 \text { Pt } 3 . \\
\text { Subtotal) }\end{array}$ \\
\hline & $\begin{array}{l}\text { Total token count } \\
\text { Mean token count }\end{array}$ & $\begin{array}{r}174,849 \\
58,283\end{array}$ \\
\hline
\end{tabular}

Table E.18 CSAC1 Probability and statistics textbook sub-corpus

\begin{tabular}{llr}
\hline Textbook Category & CSAC1 (175k target) & \multicolumn{2}{c}{ Tokens } \\
\hline Probability and & (Forsyth, 2018) Ch. 01 & 21,534 \\
statistics & (Forsyth, 2018) Ch. 02 & 32,787 \\
& (Forsyth, 2018) Ch. 03 & 33,583 \\
& (Forsyth, 2018) Ch. 04 & 58,854 \\
& (Bertsekas \& Tsisiklis, 2008) Ch. 01 & 12,949 \\
& (Bertsekas \& Tsisiklis, 2008) Ch. 02 & 9,273 \\
& \multicolumn{2}{c}{ (Bertsekas \& Tsisiklis, 2008) Ch. 03 } \\
\cline { 2 - 3 } & Total token count: & 178,290 \\
\hline & Mean token count: & 25,470 \\
\hline
\end{tabular}


Table E.19 CSAC1 Programming textbook sub-corpus

\begin{tabular}{llr}
\hline Textbook Category & CSAC1 (175k target) & \multicolumn{2}{c}{ Tokens } \\
\hline Programming & (Liang, 2017) Ch. 01 & 9,584 \\
& (Liang, 2017) Ch. 02 & 12,787 \\
& (Liang, 2017) Ch. 03 & 10,680 \\
& (Liang, 2017) Ch. 04 & 10,827 \\
& (Liang, 2017) Ch. 05 & 11,928 \\
& (Liang, 2017) Ch. 06 & 11,560 \\
& (Liang, 2017) Ch. 07 & 12,172 \\
& (Liang, 2017) Ch. 08 & 7,452 \\
& (Liang, 2017) Ch. 09 & 12,484 \\
& (Liang, 2017) Ch. 10 & 14,236 \\
& (Liang, 2017) Ch. 11 & 12,340 \\
& (Liang, 2017) Ch. 12 & 14,254 \\
& (Liang, 2017) Ch. 13 & 12,557 \\
& (Liang, 2017) Ch. 14 & 13,906 \\
& (Liang, 2017) Ch. 15 & 13,257 \\
\cline { 2 - 2 } & \multicolumn{2}{c}{ Total token count: } \\
Mean token count: & 180,024 \\
& & 12,002 \\
\hline
\end{tabular}

Table E.20 CSAC1 Computer system organization textbook sub-corpus

\begin{tabular}{llr}
\hline Textbook Category & CSAC1 (175k target) & Tokens \\
\hline Computer system & (Bryant \& O'Hallaron, 2016) Ch. 01 & 10,287 \\
organization & (Bryant \& O'Hallaron, 2016) Ch. 02 & 38,858 \\
& (Bryant \& O'Hallaron, 2016) Ch. 03 & 53,648 \\
& (Bryant \& O'Hallaron, 2016) Ch. 04 & 45,230 \\
& (Bryant \& O'Hallaron, 2016) Ch. 05 & 27,578 \\
\cline { 2 - 3 } & Total token count: & 175,601 \\
& Mean token count: & 35,120 \\
\hline
\end{tabular}


Table E.21 CSAC1 Software design textbook sub-corpus

\begin{tabular}{llr}
\hline $\begin{array}{l}\text { Textbook } \\
\text { Category }\end{array}$ & CSAC1 (175k target) & \multicolumn{1}{c}{ Tokens } \\
\hline Software design & (Sommerville, 2015) Ch. 01 & 9,870 \\
& (Sommerville, 2015) Ch. 02 & 9,698 \\
& (Sommerville, 2015) Ch. 03 & 11,239 \\
& (Sommerville, 2015) Ch. 04 & 13,634 \\
& (Sommerville, 2015) Ch. 05 & 8,862 \\
& (Sommerville, 2015) Ch. 06 & 9,251 \\
& (Sommerville, 2015) Ch. 07 & 10,520 \\
& (Sommerville, 2015) Ch. 08 & 10,857 \\
& (Sommerville, 2015) Ch. 09 & 10,580 \\
& (Sommerville, 2015) Ch. 10 & 7,850 \\
& (Sommerville, 2015) Ch. 11 & 12,397 \\
& (Sommerville, 2015) Ch. 12 & 12,685 \\
& (Sommerville, 2015) Ch. 13 & 13,050 \\
& (Sommerville, 2015) Ch. 14 & 10,609 \\
& (Sommerville, 2015) Ch. 15 & 9,355 \\
& (Sommerville, 2015) Ch. 16 & 9,541 \\
\hline & (Sommerville, 2015) Ch. 17 & 10,817 \\
\cline { 2 - 3 } Total token count: & 180,815 \\
& Mean token count: & 10,636 \\
\hline
\end{tabular}

Table E.22 CSAC1 Theory of computation textbook sub-corpus

\begin{tabular}{llr}
\hline Textbook Category & CSAC1 (175k target) & \multicolumn{1}{r}{ Tokens } \\
\hline Theory of & (Sipser, 2012) Ch. 00 & 8,730 \\
computation & (Sipser, 2012) Ch. 01 & 21,601 \\
& (Sipser, 2012) Ch. 02 & 23,054 \\
& (Sipser, 2012) Ch. 03 & 9,901 \\
& (Sipser, 2012) Ch. 04 & 7,522 \\
& (Sipser, 2012) Ch. 05 & 10,077 \\
& (Sipser, 2012) Ch. 06 & 9,914 \\
& (Sipser, 2012) Ch. 07 & 20,364 \\
& (Sipser, 2012) Ch. 08 & 12,257 \\
& (Sipser, 2012) Ch. 09 & 11,224 \\
& (Sipser, 2012) Ch. 10 & 18,997 \\
& (Barak, 2019) Ch. 01 & 11,680 \\
& (Barak, 2019) Ch. 02 & 8,858 \\
\cline { 2 - 3 } & \multicolumn{2}{c}{ Total token count: } \\
Mean token count: & 174,179 \\
& & 13,398 \\
\hline
\end{tabular}


Table E.23 CSAC2 Computer system organization ACM article sub-corpus

\begin{tabular}{|c|c|c|}
\hline Article Category & CSAC2 (35k target) & Tokens \\
\hline \multirow{5}{*}{$\begin{array}{l}\text { Computer system } \\
\text { organization }\end{array}$} & (Blem et al., 2015) & 10,165 \\
\hline & (Salfner et al., 2010) & 15,626 \\
\hline & (Talla et al., 2017) & 10,291 \\
\hline & Total token count: & 36,082 \\
\hline & Mean token count: & 12,027 \\
\hline
\end{tabular}

Table E.24 CSAC2 Computing methodologies ACM article sub-corpus

\begin{tabular}{llr}
\hline Article Category & CSAC2 (35k target) & Tokens \\
\hline Computing & (Chang \& Lin, 2011) & 4,942 \\
methodologies & (Chao et al., 2010) & 4,086 \\
& (D’Eon \& Irving, 2011) & 7,318 \\
& (Kalogerakis et al., 2010) & 6,971 \\
& (Rendle, 2012) & 6,722 \\
& (Wadhwa et al., 2013) & 5,943 \\
\cline { 2 - 3 } & \multicolumn{2}{c}{ Total token count: } \\
Mean token count: & 35,982 \\
&
\end{tabular}

Table E.25 CSAC2 Hardware ACM article sub-corpus

\begin{tabular}{|c|c|c|}
\hline Article Category & CSAC2 (35k target) & Tokens \\
\hline \multirow{6}{*}{ Hardware } & (Huang et al., 2014) & 7,654 \\
\hline & (Maimone et al., 2014) & 9,585 \\
\hline & (McLaughlin et al., 2009) & 4,698 \\
\hline & (Valiant, 2009) & 10,757 \\
\hline & (Zhao et al., 2009) & 4,108 \\
\hline & $\begin{array}{l}\text { Total token count: } \\
\text { Mean token count: }\end{array}$ & $\begin{array}{r}36,802 \\
7,360\end{array}$ \\
\hline
\end{tabular}


Table E.26 CSAC2 Human centered computing ACM article sub-corpus

\begin{tabular}{|c|c|c|}
\hline Article Category & CSAC2 (35k target) & Tokens \\
\hline \multirow{7}{*}{$\begin{array}{l}\text { Human centered } \\
\text { computing }\end{array}$} & (Harper \& Konstan, 2015) & 6,073 \\
\hline & (Hiltz et al., 2011) & 2,922 \\
\hline & (Lemos et al., 2015) & 14,587 \\
\hline & (Long et al., 2014) & 6,697 \\
\hline & (Merrell et al., 2011) & 5,136 \\
\hline & Total token count: & 35,415 \\
\hline & Mean token count: & 7,083 \\
\hline
\end{tabular}

Table E.27 CSAC2 Information systems ACM article sub-corpus

\begin{tabular}{llr}
\hline Article Category & CSAC2 (35k target) & Tokens \\
\hline Information & (Guidotti et al., 2018) & 19,847 \\
systems & (Lim et al., 2013) & 4,456 \\
& (Rodeh et al., 2013) & 11,395 \\
\cline { 2 - 3 } & \multicolumn{2}{c}{ Total token count: } \\
& Mean token count: & 11,899 \\
\hline
\end{tabular}

Table E.28 CSAC2 Mathematics of computing ACM article sub-corpus

\begin{tabular}{|c|c|c|}
\hline Article Category & CSAC2 (35k target) & Tokens \\
\hline \multirow{7}{*}{$\begin{array}{l}\text { Mathematics of } \\
\text { computing }\end{array}$} & (Bickel et al., 2010) & 5,999 \\
\hline & (Dow et al., 2010) & 5,892 \\
\hline & (Dunlavy et al., 2011) & 8,725 \\
\hline & (Hildebrandt et al., 2011) & 7,481 \\
\hline & (Schumaker \& Chen, 2009) & 7,121 \\
\hline & Total token count: & 35,218 \\
\hline & Mean token count: & 7,044 \\
\hline
\end{tabular}

Table E.29 CSAC2 Networks ACM article sub-corpus

\begin{tabular}{llr}
\hline Article Category & CSAC2 (35k target) & Tokens \\
\hline Networks & (Gomez-Uribe \& Hunt, 2015) & 9,330 \\
& (Krause \& Guestrin, 2011) & 8,214 \\
& (Manshaei et al., 2013) & 17,488 \\
\cline { 2 - 3 } & Total token count: & 35,032 \\
& Mean token count: & 11,677 \\
\hline
\end{tabular}


Table E.30 CSAC2 Security and privacy ACM article sub-corpus

\begin{tabular}{llr}
\hline Article Category & CSAC2 (35k target) & Tokens \\
\hline Security & (Alcantara et al., 2009) & 8,072 \\
and privacy & (Pék et al., 2013) & 14,926 \\
& (Shay et al., 2016) & 12,757 \\
\cline { 2 - 3 } & \multicolumn{2}{r}{ Total token count: } \\
Mean token count: & 11,755 \\
\hline
\end{tabular}

Table E.31 CSAC2 Software and its engineering ACM article sub-corpus

\begin{tabular}{llr}
\hline Article Category & CSAC2 (35k target) & Tokens \\
\hline Software and its & (Hayes, 2011) & 9,919 \\
engineering & (Peter et al., 2015) & 12,717 \\
& (Shaer \& Jacob, 2009) & 13,409 \\
\cline { 2 - 3 } & Total token count: & 36,045 \\
& Mean token count: & 12,015 \\
\hline
\end{tabular}

Table E.32 CSAC2 Theory of computation ACM article sub-corpus

\begin{tabular}{llr}
\hline Article Category & CSAC2 (35k target) & Tokens \\
\hline Theory of & (Guo et al., 2015) & 5,249 \\
computation & (He et al., 2012) & 8,171 \\
& (Kazhdan \& Hoppe, 2013) & 6,714 \\
& (Li et al., 2010) & 3,777 \\
& (Rivers et al., 2010) & 5,914 \\
& \multicolumn{2}{c}{ (Talton et al., 2011) } \\
\cline { 2 - 3 } & \multicolumn{2}{c}{ Total token count: } \\
\hline
\end{tabular}

Table E.33 CSAC2 Algorithms textbook sub-corpus

\begin{tabular}{llr}
\hline Textbook Category & CSAC2 (35k target) & Tokens \\
\hline Algorithms & (Kleinberg \& Tardos, 2005) Ch. 02 & 17,905 \\
& (Kleinberg \& Tardos, 2005) Ch. 03 & 17,266 \\
\cline { 2 - 3 } & Total token count: & 35,171 \\
& Mean token count: & 17,586 \\
\hline
\end{tabular}


Table E.34 CSAC2 Data structures textbook sub-corpus

\begin{tabular}{llr}
\hline Textbook Category & CSAC2 (35k target) & Tokens \\
\hline Data structures & (Carrano \& Henry, 2012) Ch. 01 & 13,411 \\
& (Carrano \& Henry, 2012) Ch. 01B & 5,929 \\
& (Carrano \& Henry, 2012) Ch. 02 & 16,375 \\
\cline { 2 - 3 } & Total token count: & 35,715 \\
& Mean token count: & 11,905 \\
\hline
\end{tabular}

Table E.35 CSAC2 Human centered computing textbook sub-corpus

\begin{tabular}{llr}
\hline Textbook Category & CSAC2 (35k target) & Tokens \\
\hline Human centered & (Reynolds, 2014) Ch. 02 & 16,064 \\
computing & (Reynolds, 2014) Ch. 04 & 19,345 \\
\cline { 2 - 3 } & \multicolumn{2}{c}{ Total token count: } \\
& Mean token count: & 35,409 \\
& & 17,705 \\
\hline
\end{tabular}

Table E.36 CSAC2 Mathematics of computing textbook sub-corpus

\begin{tabular}{|c|c|c|}
\hline Textbook Category & CSAC2 (35k target) & Tokens \\
\hline \multirow{6}{*}{$\begin{array}{l}\text { Mathematics of } \\
\text { computing }\end{array}$} & (Lehman et al., 2017) Ch. 01 & 7,170 \\
\hline & (Lehman et al., 2017) Ch. 03 & 12,249 \\
\hline & (Lehman et al., 2017) Ch. 04 & 8,439 \\
\hline & (Lehman et al., 2017) Ch. 05 & 8,448 \\
\hline & Total token count: & 36,306 \\
\hline & Mean token count: & 9,077 \\
\hline
\end{tabular}

Table E.37 CSAC2 Operating systems textbook sub-corpus

\begin{tabular}{llr}
\hline Textbook Category & CSAC2 (35k target) & Tokens \\
\hline Operating systems & (Anderson \& Dahlin, 2012) Ch. 02 & 19,645 \\
& (Anderson \& Dahlin, 2012) Ch. 04 & 14,685 \\
\cline { 2 - 3 } & Total token count: & 34,330 \\
& Mean token count: & 17,165 \\
\hline
\end{tabular}


Table E.38 CSAC2 Probability and statistics textbook sub-corpus

\begin{tabular}{llr}
\hline Textbook Category & CSAC2 (35k target) & Tokens \\
\hline Probability and & (Devore, 2015) Ch.01 & 21,250 \\
statistics & (Devore, 2015) Ch.02_1 to 2_4 & 16,074 \\
\cline { 2 - 3 } & \multicolumn{2}{c}{ Total token count: } \\
Mean token count: & 37,324 \\
& & 18,662 \\
\hline
\end{tabular}

Table E.39 CSAC2 Programming textbook sub-corpus

\begin{tabular}{|c|c|c|}
\hline Textbook Category & CSAC2 (35k target) & Tokens \\
\hline \multirow{7}{*}{ Programming } & (Kernighan \& Ritchie, 1988) Ch. 01 & 9,518 \\
\hline & (Kernighan \& Ritchie, 1988) Ch. 02 & 5,898 \\
\hline & (Kernighan \& Ritchie, 1988) Ch. 03 & 3,406 \\
\hline & (Kernighan \& Ritchie, 1988) Ch. 04 & 7,623 \\
\hline & (Kernighan \& Ritchie, 1988) Ch. 05 & 9,327 \\
\hline & Total token count: & 35,772 \\
\hline & Mean token count: & 7,154 \\
\hline
\end{tabular}

Table E.40 CSAC2 Software design textbook sub-corpus

\begin{tabular}{|c|c|c|}
\hline $\begin{array}{l}\text { Textbook } \\
\text { Category }\end{array}$ & CSAC2 (35k target) & Tokens \\
\hline \multirow[t]{8}{*}{ Software Design } & (Miles \& Pilone, 2008) Ch. 02 & 7,609 \\
\hline & (Miles \& Pilone, 2008) Ch. 03 & 6,894 \\
\hline & (Miles \& Pilone, 2008) Ch. 04 & 5,683 \\
\hline & (Miles \& Pilone, 2008) Ch. 05 & 3,893 \\
\hline & (Miles \& Pilone, 2008) Ch. 06 & 7,899 \\
\hline & (Miles \& Pilone, 2008) Ch. 06B & 2,906 \\
\hline & Total token count: & 34,884 \\
\hline & Mean token count: & 5,814 \\
\hline
\end{tabular}

Table E.41 CSAC2 Computer system organization textbook sub-corpus

\begin{tabular}{llr}
\hline Textbook Category & CSAC2 (35k target) & Tokens \\
\hline Computer system & (Patterson \& Hennessy, 2004) Ch. 01 & 14,255 \\
organization & (Patterson \& Hennessy, 2004) Ch. 02_1 to 2_12 & 20,789 \\
\cline { 2 - 3 } & Total token count: & 35,044 \\
& Mean token count: & 17,522 \\
\hline
\end{tabular}




\section{A COMPUTER SCIENCE ACADEMIC VOCABULARY LIST}

Table E.42 CSAC2 Theory of computation textbook sub-corpus

\begin{tabular}{llr}
\hline Textbook Category & CSAC2 (35k target) & Tokens \\
\hline Theory of & (Mertens \& Moore, 2011) Ch. 03 & 22,850 \\
computation & (Mertens \& Moore, 2011) Ch. 04 & 13,874 \\
\cline { 2 - 3 } & Total token count: & 36,724 \\
& Mean token count: & 18,362 \\
\hline
\end{tabular}


Appendix F: $\quad$ Modified End-of-line Hyphenation Removal Perl Script

[based on the original Perl script by Micher (2012)] 


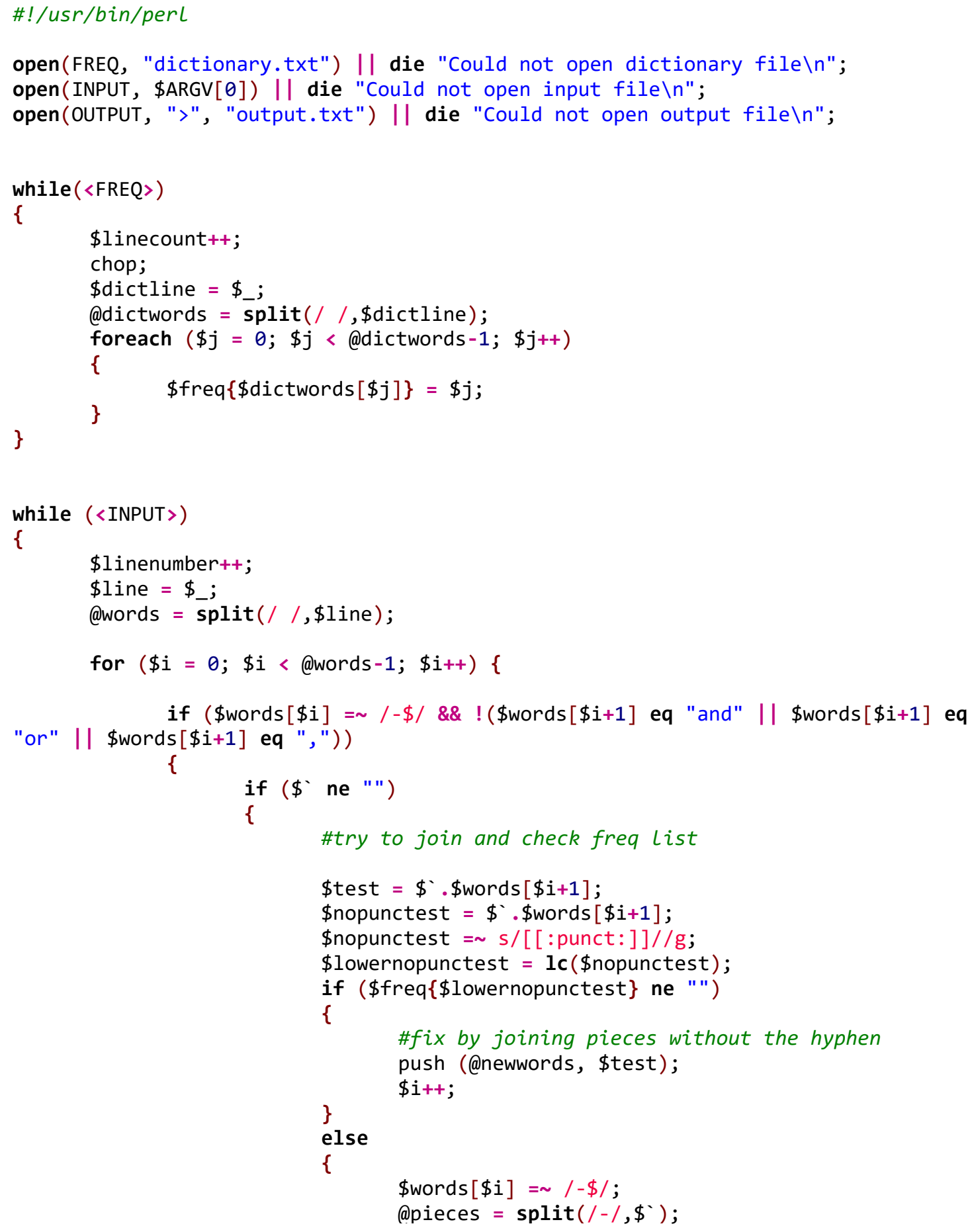




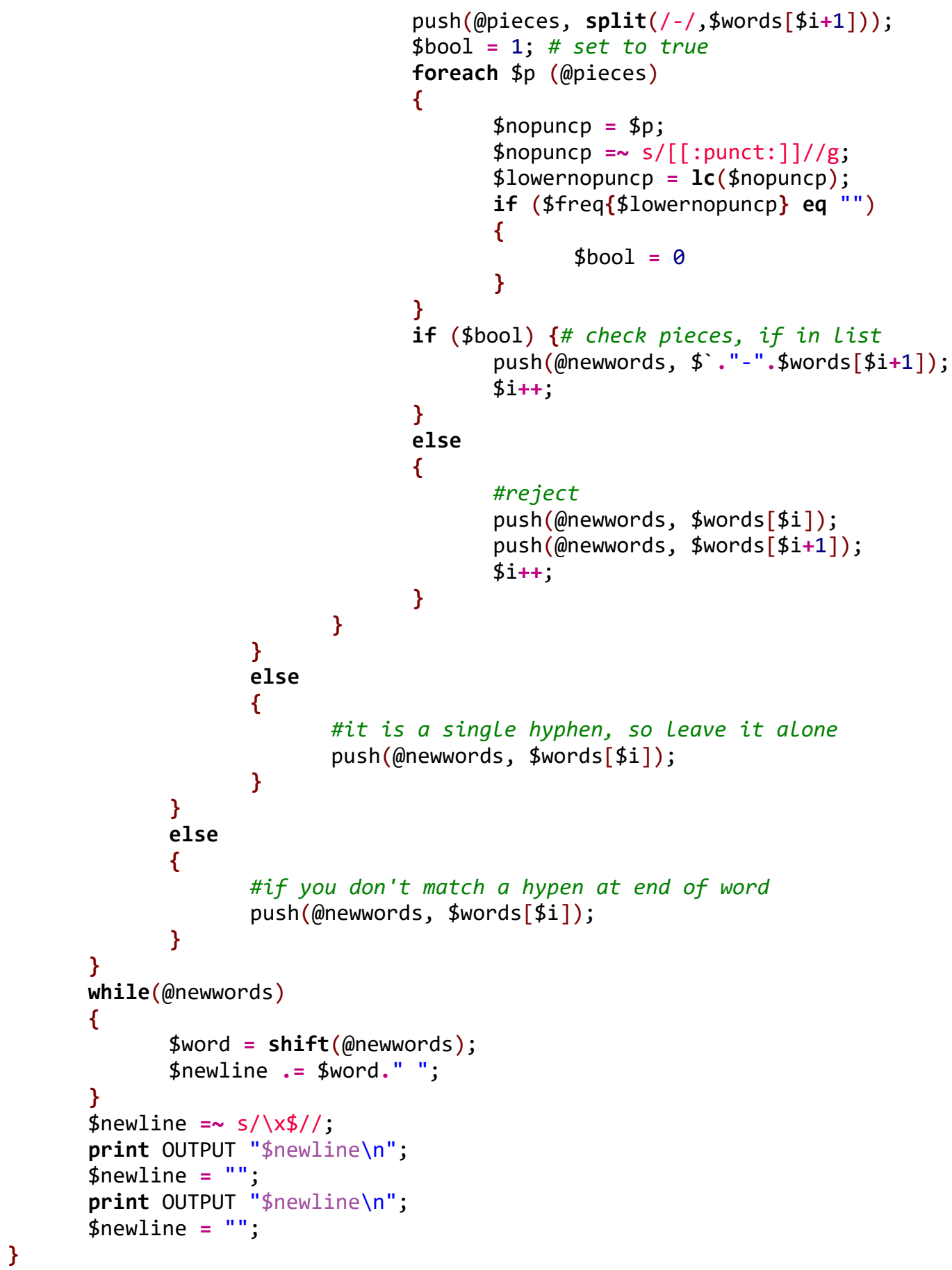


A COMPUTER SCIENCE ACADEMIC VOCABULARY LIST

Appendix G: $\quad$ ACM Citation Removal Program C++ Code 


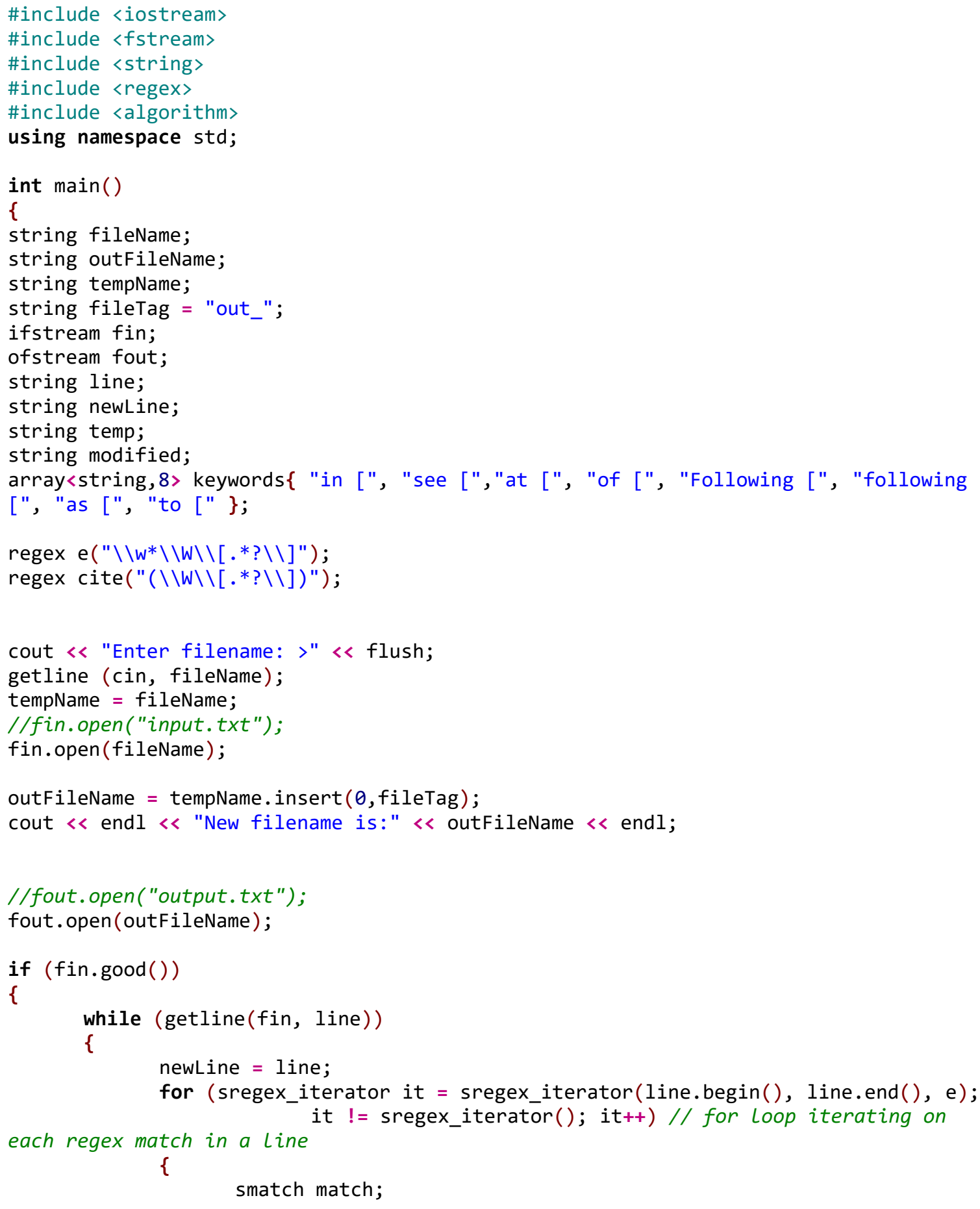




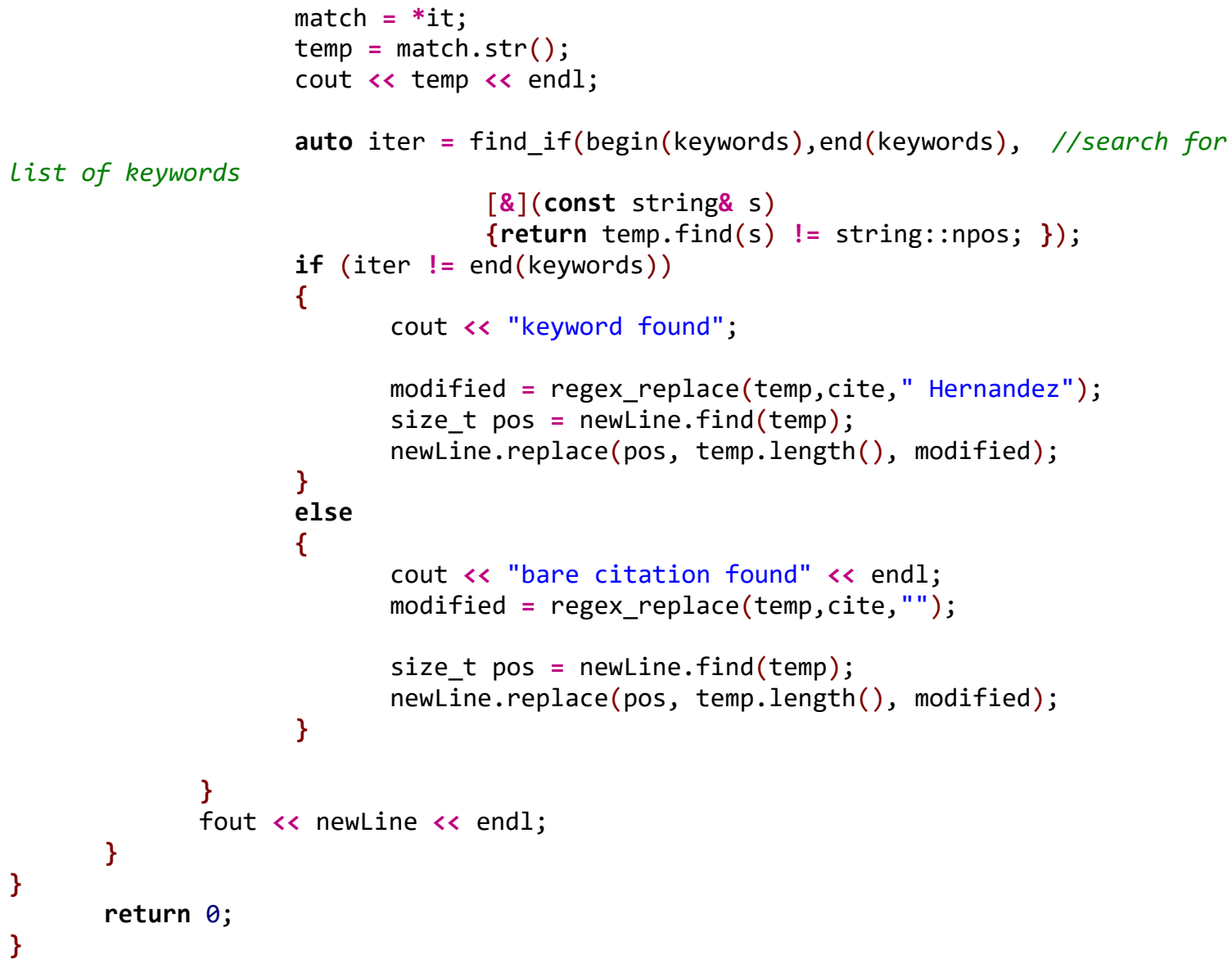




\section{Appendix H: $\quad$ CSAC1 Bibliography}

\section{CSAC1 Computer systems organization (ACM journal articles)}

Alsaedi, N., Burnap, P., \& Rana, O. (2017). Can we predict a riot? Disruptive event detection using Twitter. ACM Transactions on Internet Technology, 17(2), 1-26. https://doi.org/10.1145/2996183

Ardagna, C. A., Asal, R., Damiani, E., \& Vu, Q. H. (2015). From security to assurance in the cloud: A survey. ACM Computing Surveys, 48(1), 1-50. https://doi.org/10.1145/2767005

Bleiholder, J., \& Naumann, F. (2008). Data fusion. ACM Computing Surveys, 41(1), 141. https://doi.org/10.1145/1456650.1456651

Bulling, A., Blanke, U., \& Schiele, B. (2014). A tutorial on human activity recognition using body-worn inertial sensors. ACM Computing Surveys, 46(3), 1-33. https://doi.org/10.1145/2499621

Davis, R. I., \& Burns, A. (2011). A survey of hard real-time scheduling for multiprocessor systems. ACM Computing Surveys, 43(4), 1-44. https://doi.org/10.1145/1978802.1978814

Deng, Y. (2011). What is the future of disk drives, death or rebirth? ACM Computing Surveys, 43(3), 1-27. https://doi.org/10.1145/1922649.1922660 
Dietrich, I., \& Dressler, F. (2009). On the lifetime of wireless sensor networks. ACM Transactions on Sensor Networks, 5(1), 1-39. https://doi.org/10.1145/1464420.1464425

Fung, W. W. L., Sham, I., Yuan, G., \& Aamodt, T. M. (2009). Dynamic warp formation: Efficient MIMD control flow on SIMD graphics hardware. ACM Transactions on Architecture and Code Optimization, 6(2), 1-37. https://doi.org/10.1145/1543753.1543756

Hoffman, K., Zage, D., \& Nita-Rotaru, C. (2009). A survey of attack and defense techniques for reputation systems. ACM Computing Surveys, 42(1), 1-31. https://doi.org/10.1145/1592451.1592452

Mastelic, T., Oleksiak, A., Claussen, H., Brandic, I., Pierson, J.-M., \& Vasilakos, A. V. (2014). Cloud computing: Survey on energy efficiency. ACM Computing Surveys, 47(2), 1-36. https://doi.org/10.1145/2656204

Shuja, J., Gani, A., Bilal, K., Khan, A. U. R., Madani, S. A., Khan, S. U., \& Zomaya, A. Y. (2016). A survey of mobile device virtualization: Taxonomy and state of the art. ACM Computing Surveys, 49(1), 1-36. https://doi.org/10.1145/2897164

Suomela, J. (2013). Survey of local algorithms. ACM Computing Surveys, 45(2), 1-40. https://doi.org/10.1145/2431211.2431223

Wilson, C., Sala, A., Puttaswamy, K. P. N., \& Zhao, B. Y. (2012). Beyond Social Graphs: User Interactions in Online Social Networks and their Implications. ACM Transactions on the Web, 6(4), 1-31. https://doi.org/10.1145/2382616.2382620 


\section{CSAC1 Computing methodologies (ACM journal articles)}

Aggarwal, J. K., \& Ryoo, M. S. (2011). Human activity analysis: A review. ACM Computing Surveys, 43(3), 1-43. https://doi.org/10.1145/1922649.1922653

Barnes, C., Shechtman, E., Finkelstein, A., \& Goldman, D. B. (2009). PatchMatch: A randomized correspondence algorithm for structural image editing. ACM Transactions on Graphics, 28(3), 1. https://doi.org/10.1145/1531326.1531330

Corbett, J. C., Dean, J., Epstein, M., Fikes, A., Frost, C., Furman, J. J., ... Woodford, D. (2013). Spanner: Google’s globally distributed database. ACM Transactions on Computer Systems, 31(3), 22.

Davis, A., Rubinstein, M., Wadhwa, N., Mysore, G. J., Durand, F., \& Freeman, W. T. (2014). The visual microphone: Passive recovery of sound from video. ACM Transactions on Graphics, 33(4), 1-10. https://doi.org/10.1145/2601097.2601119

Djeu, P., Hunt, W., Wang, R., Elhassan, I., Stoll, G., \& Mark, W. R. (2011). Razor: An architecture for dynamic multiresolution ray tracing. ACM Transactions on Graphics, 30(5), 1-26. https://doi.org/10.1145/2019627.2019634

Gama, J., Žliobaitè, I., Bifet, A., Pechenizkiy, M., \& Bouchachia, A. (2014). A survey on concept drift adaptation. ACM Computing Surveys, 46(4), 1-37. https://doi.org/10.1145/2523813

Hasinoff, S. W., Sharlet, D., Geiss, R., Adams, A., Barron, J. T., Kainz, F., ... Levoy, M. (2016). Burst photography for high dynamic range and low-light imaging on mobile 
cameras. ACM Transactions on Graphics, 35(6), 1-12.

https://doi.org/10.1145/2980179.2980254

Horvath, C., \& Geiger, W. (2009). Directable, high-resolution simulation of fire on the GPU. ACM Transactions on Graphics, 28(3), 1.

https://doi.org/10.1145/1531326.1531347

Papalexakis, E. E., Faloutsos, C., \& Sidiropoulos, N. D. (2016). Tensors for data mining and data fusion: Models, applications, and scalable algorithms. ACM Transactions on Intelligent Systems and Technology, 8(2), 1-44. https://doi.org/10.1145/2915921

Pouyanfar, S., Sadiq, S., Yan, Y., Tian, H., Tao, Y., Reyes, M. P., ... Iyengar, S. S. (2018). A survey on deep learning: Algorithms, techniques, and applications. ACM Computing Surveys, 51(5), 1-36. https://doi.org/10.1145/3234150

Qi, X., \& Davison, B. D. (2009). Web page classification: Features and algorithms. ACM Computing Surveys, 41(2), 1-31. https://doi.org/10.1145/1459352.1459357

Reddy, S., Mun, M., Burke, J., Estrin, D., Hansen, M., \& Srivastava, M. (2010). Using mobile phones to determine transportation modes. ACM Transactions on Sensor Networks, 6(2), 1-27. https://doi.org/1689239.1689243

Rodríguez, N. D., Cuéllar, M. P., Lilius, J., \& Calvo-Flores, M. D. (2014). A survey on ontologies for human behavior recognition. ACM Computing Surveys, 46(4), 1-33. https://doi.org/10.1145/2523819

Silva, J. A., Faria, E. R., Barros, R. C., Hruschka, E. R., Carvalho, A. C. P. L. F. de, \& Gama, J. (2013). Data stream clustering: A survey. ACM Computing Surveys, 46(1), 1-31. https://doi.org/10.1145/2522968.2522981 
Wang, R. Y., \& Popović, J. (2009). Real-time hand-tracking with a color glove. ACM Transactions on Graphics, 28(3), 1. https://doi.org/10.1145/1531326.1531369

\section{CSAC1 Hardware (ACM journal articles)}

Aaraj, N., Raghunathan, A., \& Jha, N. K. (2008). Analysis and design of a hardware/software trusted platform module for embedded systems. ACM Transactions on Embedded Computing Systems, 8(1), 1-31. https://doi.org/10.1145/1457246.1457254

Alaghi, A., \& Hayes, J. P. (2013). Survey of stochastic computing. ACM Transactions on Embedded Computing Systems, 12(2s), 1-19. https://doi.org/10.1145/2465787.2465794

Apalkov, D., Ong, A., Driskill-Smith, A., Krounbi, M., Khvalkovskiy, A., Watts, S., ... Chen, E. (2013). Spin-transfer torque magnetic random access memory (STTMRAM). ACM Journal on Emerging Technologies in Computing Systems, 9(2), 135. https://doi.org/10.1145/2463585.2463589

Calabrese, F., Ferrari, L., \& Blondel, V. D. (2014). Urban Sensing Using Mobile Phone Network Data: A Survey of Research. ACM Computing Surveys, 47(2), 1-20. https://doi.org/10.1145/2655691

Henson, M., \& Taylor, S. (2014). Memory encryption: A survey of existing techniques. ACM Computing Surveys, 46(4), 1-26. https://doi.org/10.1145/2566673 
Lanman, D., \& Luebke, D. (2013). Near-eye light field displays. ACM Transactions on Graphics, 32(6), 1-10. https://doi.org/10.1145/2508363.2508366

Lien, J., Gillian, N., Karagozler, M. E., Amihood, P., Schwesig, C., Olson, E., ... Poupyrev, I. (2016). Soli: Ubiquitous gesture sensing with millimeter wave radar. ACM Transactions on Graphics, 35(4), 1-19. https://doi.org/10.1145/2897824.2925953

Maimone, A., Georgiou, A., \& Kollin, J. S. (2017). Holographic near-eye displays for virtual and augmented reality. ACM Transactions on Graphics, 36(4), 1-16. https://doi.org/10.1145/3072959.3073624

Medina, V., \& García, J. M. (2014). A survey of migration mechanisms of virtual machines. ACM Computing Surveys, 46(3), 1-33. https://doi.org/10.1145/2492705

Ni, K., Srivastava, M., Ramanathan, N., Chehade, M. N. H., Balzano, L., Nair, S., ... Hansen, M. (2009). Sensor network data fault types. ACM Transactions on Sensor Networks, 5(3), 1-29. https://doi.org/10.1145/1525856.1525863

Sen, S., Joe-Wong, C., Ha, S., \& Chiang, M. (2013). A survey of smart data pricing: Past proposals, current plans, and future trends. ACM Computing Surveys, 46(2), 1-37. https://doi.org/10.1145/2543581.2543582

Shin, Y., Seomun, J., Choi, K.-M., \& Sakurai, T. (2010). Power gating: Circuits, design methodologies, and best practice for standard-cell VLSI designs. ACM Transactions on Design Automation of Electronic Systems, 15(4), 1-37. https://doi.org/10.1145/1835420.1835421 
Vasilomanolakis, E., Karuppayah, S., Mühlhäuser, M., \& Fischer, M. (2015). Taxonomy and survey of collaborative intrusion detection. ACM Computing Surveys, 47(4), 133. https://doi.org/10.1145/2716260

Woodcock, J., Larsen, P. G., Bicarregui, J., \& Fitzgerald, J. (2009). Formal methods: Practice and experience. ACM Computing Surveys, 41(4), 1-36. https://doi.org/10.1145/1592434.1592436

Yang, Z., Zhou, Z., \& Liu, Y. (2013). From RSSI to CSI: Indoor localization via channel response. ACM Computing Surveys, 46(2), 1-32.

https://doi.org/10.1145/2543581.2543592

\section{CSAC1 Human centered computing (ACM journal articles)}

Bau, O., \& Poupyrev, I. (2012). REVEL: Tactile feedback technology for augmented reality. ACM Transactions on Graphics, 31(4), 1-11. https://doi.org/10.1145/2185520.2185585

Cao, C., Weng, Y., Lin, S., \& Zhou, K. (2013). 3D shape regression for real-time facial animation. ACM Transactions on Graphics, 32(4), 1. https://doi.org/10.1145/2461912.2462012

Carminati, B., Ferrari, E., \& Perego, A. (2009). Enforcing access control in Web-based social networks. ACM Transactions on Information and System Security, 13(1), 138. https://doi.org/10.1145/1609956.1609962 
Coppola, R., \& Morisio, M. (2016). Connected car: Technologies, issues, future trends. ACM Computing Surveys, 49(3), 1-36. https://doi.org/10.1145/2971482

Coros, S., Thomaszewski, B., Noris, G., Sueda, S., Forberg, M., Sumner, R. W., ... Bickel, B. (2013). Computational design of mechanical characters. ACM Transactions on Graphics, 32(4), 1. https://doi.org/10.1145/2461912.2461953

Feng, J., Lazar, J., Kumin, L., \& Ozok, A. (2010). Computer usage by children with down syndrome: Challenges and future research. ACM Transactions on Accessible Computing, 2(3), 1-44. https://doi.org/10.1145/1714458.1714460

Gerber, E. M., \& Hui, J. (2013). Crowdfunding: Motivations and deterrents for participation. ACM Transactions on Computer-Human Interaction, 20(6), 1-32. https://doi.org/10.1145/2530540

Hassenzahl, M., Heidecker, S., Eckoldt, K., Diefenbach, S., \& Hillmann, U. (2012). All You Need is Love: Current Strategies of Mediating Intimate Relationships through Technology. ACM Transactions on Computer-Human Interaction, 19(4), 1-19. https://doi.org/10.1145/2395131.2395137

Höök, K., \& Löwgren, J. (2012). Strong concepts: Intermediate-level knowledge in interaction design research. ACM Transactions on Computer-Human Interaction, 19(3), 1-18. https://doi.org/10.1145/2362364.2362371

Iriberri, A., \& Leroy, G. (2009). A life-cycle perspective on online community success. ACM Computing Surveys, 41(2), 1-29. https://doi.org/10.1145/1459352.1459356 
Lindgaard, G., Dudek, C., Sen, D., Sumegi, L., \& Noonan, P. (2011). An exploration of relations between visual appeal, trustworthiness and perceived usability of homepages. ACM Transactions on Computer-Human Interaction, 18(1), 1-30. https://doi.org/10.1145/1959022.1959023

Medhi, I., Patnaik, S., Brunskill, E., Gautama, S. N. N., Thies, W., \& Toyama, K. (2011). Designing mobile interfaces for novice and low-literacy users. ACM Transactions on Computer-Human Interaction, 18(1), 1-28.

https://doi.org/10.1145/1959022.1959024

Reinecke, K., \& Bernstein, A. (2011). Improving performance, perceived usability, and aesthetics with culturally adaptive user interfaces. ACM Transactions on ComputerHuman Interaction, 18(2), 1-29. https://doi.org/10.1145/1970378.1970382

Rosenberg, I., \& Perlin, K. (2009). The UnMousePad: An interpolating multi-touch force-sensing input pad. ACM Transactions on Graphics, 28(3), 1. https://doi.org/10.1145/1531326.1531371

Wang, D., \& Mark, G. (2015). Internet censorship in china: Examining user awareness and attitudes. ACM Transactions on Computer-Human Interaction, 22(6), 1-22. https://doi.org/10.1145/2818997

Weerasiri, D., Barukh, M. C., Benatallah, B., Sheng, Q. Z., \& Ranjan, R. (2017). A taxonomy and survey of cloud resource orchestration techniques. ACM Computing Surveys, 50(2), 1-41. https://doi.org/10.1145/3054177 


\section{CSAC1 Information systems (ACM journal articles)}

Batini, C., Cappiello, C., Francalanci, C., \& Maurino, A. (2009). Methodologies for data quality assessment and improvement. ACM Computing Surveys, 41(3), 1-52. https://doi.org/10.1145/1541880.1541883

Beatty, P., Reay, I., Dick, S., \& Miller, J. (2011). Consumer trust in e-commerce web sites: A meta-study. ACM Computing Surveys, 43(3), 1-46. https://doi.org/10.1145/1922649.1922651

Bonchi, F., Castillo, C., Gionis, A., \& Jaimes, A. (2011). Social network analysis and mining for business applications. ACM Transactions on Intelligent Systems and Technology, 2(3), 1-37. https://doi.org/10.1145/1961189.1961194

Cacheda, F., Carneiro, V., Fernández, D., \& Formoso, V. (2011). Comparison of collaborative filtering algorithms: Limitations of current techniques and proposals for scalable, high-performance recommender systems. ACM Transactions on the Web, 5(1), 1-33. https://doi.org/10.1145/1921591.1921593

Carpineto, C., \& Romano, G. (2012). A survey of automatic query expansion in information retrieval. ACM Computing Surveys, 44(1), 1-50. https://doi.org/10.1145/2071389.2071390

Carpineto, C., Osiński, S., Romano, G., \& Weiss, D. (2009). A survey of web clustering engines. ACM Computing Surveys, 41(3), 1-38.

https://doi.org/10.1145/1541880.1541884 
Mooney, C. H., \& Roddick, J. F. (2013). Sequential pattern mining-Approaches and algorithms. ACM Computing Surveys, 45(2), 1-39.

https://doi.org/10.1145/2431211.2431218

Parent, C., Pelekis, N., Theodoridis, Y., Yan, Z., Spaccapietra, S., Renso, C., ... Macedo, J. (2013). Semantic trajectories modeling and analysis. ACM Computing Surveys, 45(4), 1-32. https://doi.org/10.1145/2501654.2501656

Shaul, L., \& Tauber, D. (2013). Critical success factors in enterprise resource planning systems: Review of the last decade. ACM Computing Surveys, 45(4), 1-39. https://doi.org/10.1145/2501654.2501669

Shi, Y., Larson, M., \& Hanjalic, A. (2014). Collaborative filtering beyond the user-item matrix: A survey of the state of the art and future challenges. ACM Computing Surveys, 47(1), 1-45. https://doi.org/10.1145/2556270

Zheng, Y. (2015). Trajectory data mining: An overview. ACM Transactions on Intelligent Systems and Technology, 6(3), 1-41. https://doi.org/10.1145/2743025

\section{CSAC1 Mathematics of computing (ACM journal articles)}

Blei, D. M., Griffiths, T. L., \& Jordan, M. I. (2010). The nested chinese restaurant process and bayesian nonparametric inference of topic hierarchies. Journal of the ACM, 57(2), 1-30. https://doi.org/10.1145/1667053.1667056

Candès, E. J., Li, X., Ma, Y., \& Wright, J. (2011). Robust principal component analysis? Journal of the ACM, 58(3), 1-37. https://doi.org/10.1145/1970392.1970395 
Chentanez, N., \& Müller, M. (2011). Real-time Eulerian water simulation using a restricted tall cell grid. ACM SIGGRAPH 2011 Papers on - SIGGRAPH '11, 1. https://doi.org/10.1145/1964921.1964977

Chentanez, N., Alterovitz, R., Ritchie, D., Cho, L., Hauser, K. K., Goldberg, K., ... O’Brien, J. F. (2009). Interactive simulation of surgical needle insertion and steering. ACM Transactions on Graphics, 28(3), 1. https://doi.org/10.1145/1531326.1531394

Esling, P., \& Agon, C. (2012). Time-series data mining. ACM Computing Surveys, 45(1), 1-34. https://doi.org/10.1145/2379776.2379788

Kim, J., \& Pollard, N. S. (2011). Fast simulation of skeleton-driven deformable body characters. ACM Transactions on Graphics, 30(5), 1-19. https://doi.org/10.1145/2019627.2019640

Martínez, V., Berzal, F., \& Cubero, J.-C. (2016). A survey of link prediction in complex networks. ACM Computing Surveys, 49(4), 1-33. https://doi.org/10.1145/3012704

Moser, R. A., \& Tardos, G. (2010). A constructive proof of the general lovász local lemma. Journal of the ACM, 57(2), 1-15. https://doi.org/10.1145/1667053.1667060

Nielsen, C. B., Larsen, P. G., Fitzgerald, J., Woodcock, J., \& Peleska, J. (2015). Systems of systems engineering: Basic concepts, model-based techniques, and research directions. ACM Computing Surveys, 48(2), 1-41. https://doi.org/10.1145/2794381

Patterson, M. A., \& Rao, A. V. (2014). GPOPS-II: A MATLAB software for solving multiple-phase optimal control problems using hp-adaptive gaussian quadrature 
collocation methods and sparse nonlinear programming. ACM Transactions on Mathematical Software, 41(1), 1-37. https://doi.org/10.1145/2558904

Rao, A. V., Benson, D. A., Darby, C., Patterson, M. A., Francolin, C., Sanders, I., \& Huntington, G. T. (2010). Algorithm 902: GPOPS, A MATLAB software for solving multiple-phase optimal control problems using the gauss pseudospectral method.

ACM Transactions on Mathematical Software, 37(2), 1-39.

https://doi.org/10.1145/1731022.1731032

Schumacher, C., Bickel, B., Rys, J., Marschner, S., Daraio, C., \& Gross, M. (2015). Microstructures to control elasticity in 3D printing. ACM Transactions on Graphics, 34(4), 136:1-136:13. https://doi.org/10.1145/2766926

Si, H. (2015). Tetgen, a Delaunay-based quality tetrahedral mesh generator. ACM Transactions on Mathematical Software, 41(2), 1-36. https://doi.org/10.1145/2629697

Solenthaler, B., \& Gross, M. (2011). Two-scale particle simulation. ACM SIGGRAPH 2011 Papers on - SIGGRAPH '11, 1. https://doi.org/10.1145/1964921.1964976

Stava, O., Vanek, J., Benes, B., Carr, N., \& Měch, R. (2012). Stress relief: Improving structural strength of 3D printable objects. ACM Transactions on Graphics, 31(4), 1-11. https://doi.org/10.1145/2185520.2185544

Weber, K., Otto, B., \& Österle, H. (2009). One Size Does Not Fit All—A Contingency Approach to Data Governance. Journal of Data and Information Quality, 1(1), 1-27. https://doi.org/10.1145/1515693.1515696 
Zhou, Q., Panetta, J., \& Zorin, D. (2013). Worst-case structural analysis. ACM Transactions on Graphics, 32(4), 1. https://doi.org/10.1145/2461912.2461967

\section{CSAC1 Networks (ACM journal articles)}

Baccour, N., Koubâa, A., Mottola, L., Zúñiga, M. A., Youssef, H., Boano, C. A., \& Alves, M. (2012). Radio link quality estimation in wireless sensor networks: A survey. ACM Transactions on Sensor Networks, 8(4), 1-33. https://doi.org/10.1145/2240116.2240123

Di Francesco, M., Das, S. K., \& Anastasi, G. (2011). Data collection in wireless sensor networks with mobile elements: A survey. ACM Transactions on Sensor Networks, 8(1), 1-31. https://doi.org/10.1145/1993042.1993049

Eisenman, S. B., Miluzzo, E., Lane, N. D., Peterson, R. A., Ahn, G.-S., \& Campbell, A. T. (2009). BikeNet: A mobile sensing system for cyclist experience mapping. ACM Transactions on Sensor Networks, 6(1), 1-39.

https://doi.org/10.1145/1653760.1653766

Gaeta, R. (2018). A Model of Information Diffusion in Interconnected Online Social Networks. ACM Transactions on the Web, 12(2), 1-21. https://doi.org/10.1145/3160000

Huang, K., Siegel, M., \& Madnick, S. (2018). Systematically understanding the cyber attack business: A survey. ACM Computing Surveys, 51(4), 1-36. https://doi.org/10.1145/3199674 
Jordan, S. (2009). Implications of Internet architecture on net neutrality. ACM Transactions on Internet Technology, 9(2), 1-28. https://doi.org/10.1145/1516539.1516540

Kurian, J., \& Sarac, K. (2010). A survey on the design, applications, and enhancements of application-layer overlay networks. ACM Computing Surveys, 43(1), 1-34. https://doi.org/10.1145/1824795.1824800

Law, Y. W., Palaniswami, M., Hoesel, L. V., Doumen, J., Hartel, P., \& Havinga, P. (2009). Energy-efficient link-layer jamming attacks against wireless sensor network MAC protocols. ACM Transactions on Sensor Networks, 5(1), 1-38. https://doi.org/10.1145/1464420.1464426

Li, M., \& Liu, Y. (2009). Underground coal mine monitoring with wireless sensor networks. ACM Transactions on Sensor Networks, 5(2), 1-29. https://doi.org/10.1145/1498915.1498916

Mitchell, R., \& Chen, I.-R. (2014). A survey of intrusion detection techniques for cyberphysical systems. ACM Computing Surveys, 46(4), 1-29. https://doi.org/10.1145/2542049

Muir, J. A., \& Oorschot, P. C. V. (2009). Internet geolocation: Evasion and counterevasion. ACM Computing Surveys, 42(1), 1-23. https://doi.org/10.1145/1592451.1592455

Razzaque, M. A., Bleakley, C., \& Dobson, S. (2013). Compression in wireless sensor networks: A survey and comparative evaluation. ACM Transactions on Sensor Networks, 10(1), 1-44. https://doi.org/10.1145/2528948 
Walfish, M., Vutukuru, M., Balakrishnan, H., Karger, D., \& Shenker, S. (2010). DDoS defense by offense. ACM Transactions on Computer Systems, 28(1), 1-54. https://doi.org/10.1145/1731060.1731063

\section{CSAC1 Security and privacy (ACM journal articles)}

Abadi, M., Budiu, M., Erlingsson, Ú., \& Ligatti, J. (2009). Control-flow integrity principles, implementations, and applications. ACM Transactions on Information and System Security, 13(1), 1-40. https://doi.org/10.1145/1609956.1609960

Acquisti, A., Sleeper, M., Wang, Y., Wilson, S., Adjerid, I., Balebako, R., ... Schaub, F. (2017). Nudges for privacy and security: Understanding and assisting users’ choices online. ACM Computing Surveys, 50(3), 1-41. https://doi.org/10.1145/3054926

Biddle, R., Chiasson, S., \& Van Oorschot, P. C. (2012). Graphical passwords: Learning from the first twelve years. ACM Computing Surveys, 44(4), 1-41.

https://doi.org/10.1145/2333112.2333114

Chang, J., Venkatasubramanian, K. K., West, A. G., \& Lee, I. (2013). Analyzing and defending against web-based malware. ACM Computing Surveys, 45(4), 1-35. https://doi.org/10.1145/2501654.2501663

Diesburg, S. M., \& Wang, A.-I. A. (2010). A survey of confidential data storage and deletion methods. ACM Computing Surveys, 43(1), 1-37.

https://doi.org/10.1145/1824795.1824797 
Enck, W., Gilbert, P., Han, S., Tendulkar, V., Chun, B.-G., Cox, L. P., ... Sheth, A. N. (2014). Taintdroid: An information-flow tracking system for realtime privacy monitoring on smartphones. ACM Transactions on Computer Systems, 32(2), 1-29. https://doi.org/10.1145/2619091

Fung, B. C. M., Wang, K., Chen, R., \& Yu, P. S. (2010). Privacy-preserving data publishing: A survey of recent developments. ACM Computing Surveys, 42(4), 1-53. https://doi.org/10.1145/1749603.1749605

Heartfield, R., \& Loukas, G. (2015). A taxonomy of attacks and a survey of defence mechanisms for semantic social engineering attacks. ACM Computing Surveys, 48(3), 1-39. https://doi.org/10.1145/2835375

Liu, Y., Ning, P., \& Reiter, M. K. (2011). False data injection attacks against state estimation in electric power grids. ACM Transactions on Information and System Security, 14(1), 1-33. https://doi.org/10.1145/1952982.1952995

Rodríguez-Gómez, R. A., Maciá-Fernández, G., \& García-Teodoro, P. (2013). Survey and taxonomy of botnet research through life-cycle. ACM Computing Surveys, 45(4), 1-33. https://doi.org/10.1145/2501654.2501659

Roemer, R., Buchanan, E., Shacham, H., \& Savage, S. (2012). Return-oriented programming: Systems, languages, and applications. ACM Transactions on Information and System Security, 15(1), 1-34.

https://doi.org/10.1145/2133375.2133377 
Ye, Y., Li, T., Adjeroh, D., \& Iyengar, S. S. (2017). A survey on malware detection using data mining techniques. ACM Computing Surveys, 50(3), 1-40.

https://doi.org/10.1145/3073559

\section{CSAC1 Software and it engineering (ACM journal articles)}

Cockburn, A., Karlson, A., \& Bederson, B. B. (2008). A review of overview+detail, zooming, and focus+context interfaces. ACM Computing Surveys, 41(1), 1-31. https://doi.org/10.1145/1456650.1456652

Harman, M., Mansouri, S. A., \& Zhang, Y. (2012). Search-based software engineering: Trends, techniques and applications. ACM Computing Surveys, 45(1), 1-61. https://doi.org/10.1145/2379776.2379787

Ko, A. J., Myers, B., Rosson, M. B., Rothermel, G., Shaw, M., Wiedenbeck, S., ... Lieberman, H. (2011). The state of the art in end-user software engineering. ACM Computing Surveys, 43(3), 1-44. https://doi.org/10.1145/1922649.1922658

Largent, D. L. (2016). Measuring and understanding team development by capturing selfassessed enthusiasm and skill levels. ACM Transactions on Computing Education, 16(2), 1-27. https://doi.org/10.1145/2791394

Lopes, C. V., Maj, P., Martins, P., Saini, V., Yang, D., Zitny, J., ... Vitek, J. (2017). DéjàVu: A map of code duplicates on GitHub. Proceedings of the ACM on Programming Languages, 1(OOPSLA), 1-28. https://doi.org/10.1145/3133908 
Mendling, J., Dustdar, S., Gal, A., García-Bañuelos, L., Governatori, G., Hull, R., ... Dumas, M. (2018). Blockchains for business process management-Challenges and opportunities. ACM Transactions on Management Information Systems, 9(1), 1-16. https://doi.org/10.1145/3183367

Mili, H., Tremblay, G., Jaoude, G. B., Lefebvre, É., Elabed, L., \& Boussaidi, G. E. (2010). Business process modeling languages: Sorting through the alphabet soup. ACM Computing Surveys, 43(1), 1-56. https://doi.org/10.1145/1824795.1824799

Mottola, L., \& Picco, G. P. (2011). Programming wireless sensor networks: Fundamental concepts and state of the art. ACM Computing Surveys, 43(3), 1-51. https://doi.org/10.1145/1922649.1922656

Ouyang, C., Dumas, M., Aalst, W. M. P. V. D., Hofstede, A. H. M. T., \& Mendling, J. (2009). From business process models to process-oriented software systems. ACM Transactions on Software Engineering and Methodology, 19(1), 1-37. https://doi.org/10.1145/1555392.1555395

Shahriar, H., \& Zulkernine, M. (2012). Mitigating program security vulnerabilities: Approaches and challenges. ACM Computing Surveys, 44(3), 1-46. https://doi.org/10.1145/2187671.2187673

Stefik, A., \& Siebert, S. (2013). An empirical investigation into programming language syntax. ACM Transactions on Computing Education, 13(4), 1-40. https://doi.org/10.1145/2534973 
Toosi, A. N., Calheiros, R. N., \& Buyya, R. (2014). Interconnected cloud computing environments: Challenges, taxonomy, and survey. ACM Computing Surveys, 47(1), 1-47. https://doi.org/10.1145/2593512

\section{CSAC1 Theory of computation (ACM journal articles)}

Arora, S., Rao, S., \& Vazirani, U. (2009). Expander flows, geometric embeddings and graph partitioning. Journal of the ACM, 56(2), 1-37. https://doi.org/10.1145/1502793.1502794

Bermano, A., Vaxman, A., \& Gotsman, C. (2011). Online reconstruction of 3D objects from arbitrary cross-sections. ACM Transactions on Graphics, 30(5), 1-11. https://doi.org/10.1145/2019627.2019632

Bokeloh, M., Wand, M., \& Seidel, H.-P. (2010). A connection between partial symmetry and inverse procedural modeling. ACM Transactions on Graphics, 29(4), 1. https://doi.org/10.1145/1778765.1778841

Bommes, D., Zimmer, H., \& Kobbelt, L. (2009). Mixed-integer quadrangulation. ACM Transactions on Graphics, 28(3), 1. https://doi.org/10.1145/1531326.1531383

Calì, J., Calian, D. A., Amati, C., Kleinberger, R., Steed, A., Kautz, J., \& Weyrich, T. (2012). 3D-printing of non-assembly, articulated models. ACM Transactions on Graphics, 31(6), 1. https://doi.org/10.1145/2366145.2366149 
Cederman, D., \& Tsigas, P. (2009). GPU-Quicksort: A practical Quicksort algorithm for graphics processors. Journal of Experimental Algorithmics, 14, 1.4.

https://doi.org/10.1145/1498698.1564500

Farbman, Z., Hoffer, G., Lipman, Y., Cohen-Or, D., \& Lischinski, D. (2009).

Coordinates for instant image cloning. ACM Transactions on Graphics, 28(3), 1. https://doi.org/10.1145/1531326.1531373

Gal, R., Sorkine, O., Mitra, N. J., \& Cohen-Or, D. (2009). iWIRES: An analyze-and-edit approach to shape manipulation. ACM Transactions on Graphics, 28(3), 1. https://doi.org/10.1145/1531326.1531339

Gao, W., Tian, Y., Huang, T., \& Yang, Q. (2010). Vlogging: A survey of videoblogging technology on the web. ACM Computing Surveys, 42(4), 1-57.

https://doi.org/10.1145/1749603.1749606

Jung, R., Jourdan, J.-H., Krebbers, R., \& Dreyer, D. (2017). RustBelt: Securing the foundations of the rust programming language. Proceedings of the ACM on Programming Languages, 2(POPL), 1-34. https://doi.org/10.1145/3158154

Martin, S., Kaufmann, P., Botsch, M., Grinspun, E., \& Gross, M. (2010). Unified simulation of elastic rods, shells, and solids. ACM Transactions on Graphics, 29(4), 1. https://doi.org/10.1145/1778765.1778776

Palubicki, W., Horel, K., Longay, S., Runions, A., Lane, B., Měch, R., \& Prusinkiewicz, P. (2009). Self-organizing tree models for image synthesis. ACM Transactions on Graphics, 28(3), 1. https://doi.org/10.1145/1531326.1531364 
Shankar, N. (2009). Automated deduction for verification. ACM Computing Surveys, 41(4), 1-56. https://doi.org/10.1145/1592434.1592437

Shao, T., Xu, W., Zhou, K., Wang, J., Li, D., \& Guo, B. (2012). An interactive approach to semantic modeling of indoor scenes with an RGBD camera. ACM Transactions on Graphics, 31(6), 1. https://doi.org/10.1145/2366145.2366155

Tagliasacchi, A., Zhang, H., \& Cohen-Or, D. (2009). Curve skeleton extraction from incomplete point cloud. ACM Transactions on Graphics, 28(3), 1. https://doi.org/10.1145/1531326.1531377

Wang, W., Wang, T. Y., Yang, Z., Liu, L., Tong, X., Tong, W., ... Liu, X. (2013). Costeffective printing of 3D objects with skin-frame structures. ACM Transactions on Graphics, 32(6), 1-10. https://doi.org/10.1145/2508363.2508382

Wei, X., \& Chai, J. (2010). VideoMocap: Modeling physically realistic human motion from monocular video sequences. ACM Transactions on Graphics, 29(4), 1. https://doi.org/10.1145/1778765.1778779

Zhu, L., Xu, W., Snyder, J., Liu, Y., Wang, G., \& Guo, B. (2012). Motion-guided mechanical toy modeling. ACM Transactions on Graphics, 31(6), 1. https://doi.org/10.1145/2366145.2366146

\section{CSAC1 Algorithms (Textbooks)}

Cormen, T. H., Leiserson, C. E., Rivest, R. L., \& Stein, C. (2009). Introduction to algorithms. MIT Press. 


\section{CSAC1 Data structures (Textbooks)}

Weiss, M. A. (2011). Data structures and algorithm analysis in Java. Pearson Higher Ed.

\section{CSAC1 Human centered computing (Textbooks)}

Quinn, M. J. (2016). Ethics for the information age. Pearson Education.

\section{CSAC1 Operating systems (Textbooks)}

Arpaci-Dusseau, R. H., \& Arpaci-Dusseau, A. C. (2018). Operating systems: Three easy pieces. CreateSpace Independent Publishing Platform.

\section{CSAC1 Mathematics of computing (Textbooks)}

Rosen, K. (2011). Discrete mathematics and its applications. McGraw-Hill Higher Education. 


\section{CSAC1 Computer systems organization (Textbooks)}

Bryant, R. E., \& O’Hallaron, D. R. (2016). Computer systems: A programmer’s perspective. Pearson.

\section{CSAC1 Probability and statistics (Textbooks)}

Bertsekas, D. P., \& Tsitsiklis, J. N. (2008). Introduction to probability. Athena Scientific.

Forsyth, D. (2017). Probability and statistics for computer science. Springer.

\section{CSAC1 Software design (Textbooks)}

Sommerville, I. (2015). Software engineering. Pearson.

\section{CSAC1 Programming (Textbooks)}

Liang, Y. D. (2018). Introduction to Java programming and data structures. Pearson Education. 


\section{CSAC1 Theory of computation (Textbooks)}

Sipser, M. (2012). Introduction to the theory of computation. Cengage Learning. 


\section{Appendix I: $\quad$ CSAC2 Bibliography}

\section{CSAC2 Computer systems organization (ACM journal articles)}

Blem, E., Menon, J., Vijayaraghavan, T., \& Sankaralingam, K. (2015). ISA wars:

Understanding the relevance of isa being RISC or CISC to performance, power, and energy on modern architectures. ACM Transactions on Computer Systems, 33(1), 134. https://doi.org/10.1145/2699682

Salfner, F., Lenk, M., \& Malek, M. (2010). A survey of online failure prediction methods. ACM Computing Surveys, 42(3), 1-42.

https://doi.org/10.1145/1670679.1670680

Talla, V., Kellogg, B., Gollakota, S., \& Smith, J. R. (2017). Battery-free cellphone. Proceedings of the ACM on Interactive, Mobile, Wearable and Ubiquitous Technologies, 1(2), 1-20. https://doi.org/10.1145/3090090

\section{CSAC2 Computing methodologies (ACM journal articles)}

Chang, C.-C., \& Lin, C.-J. (2011). LIBSVM: A library for support vector machines. ACM Transactions on Intelligent Systems and Technology, 2(3), 1-27. https://doi.org/10.1145/1961189.1961199

Chao, I., Pinkall, U., Sanan, P., \& Schröder, P. (2010). A simple geometric model for elastic deformations. ACM Transactions on Graphics, 29(4), 1. https://doi.org/10.1145/1778765.1778775 
D’Eon, E., \& Irving, G. (2011). A quantized-diffusion model for rendering translucent materials. ACM SIGGRAPH 2011 Papers on - SIGGRAPH '11, 1. https://doi.org/10.1145/1964921.1964951

Kalogerakis, E., Hertzmann, A., \& Singh, K. (2010). Learning 3D mesh segmentation and labeling. ACM Transactions on Graphics, 29(4), 1. https://doi.org/10.1145/1778765.1778839

Rendle, S. (2012). Factorization machines with libFM. ACM Transactions on Intelligent Systems and Technology, 3(3), 1-22. https://doi.org/10.1145/2168752.2168771

Wadhwa, N., Rubinstein, M., Durand, F., \& Freeman, W. T. (2013). Phase-based video motion processing. ACM Transactions on Graphics, 32(4), 1.

https://doi.org/10.1145/2461912.2461966

\section{CSAC2 Hardware (ACM journal articles)}

Huang, F.-C., Wetzstein, G., Barsky, B. A., \& Raskar, R. (2014). Eyeglasses-free display: Towards correcting visual aberrations with computational light field displays. ACM Transactions on Graphics, 33(4), 1-12. https://doi.org/10.1145/2601097.2601122

Maimone, A., Lanman, D., Rathinavel, K., Keller, K., Luebke, D., \& Fuchs, H. (2014). Pinlight displays: Wide field of view augmented reality eyeglasses using defocused point light sources. ACM Transactions on Graphics, 33(4), 1-11. https://doi.org/10.1145/2601097.2601141 
McLaughlin, A. C., Rogers, W. A., \& Fisk, A. D. (2009). Using direct and indirect input devices: Attention demands and age-related differences. ACM Transactions on Computer-Human Interaction, 16(1), 1-15. https://doi.org/10.1145/1502800.1502802

Valiant, L. G. (2009). Evolvability. Journal of the ACM, 56(1), 1-21. https://doi.org/10.1145/1462153.1462156

Zhao, W., Belhaire, E., Chappert, C., \& Mazoyer, P. (2009). Spin transfer torque (STT)MRAM--based runtime reconfiguration FPGA circuit. ACM Transactions on Embedded Computing Systems, 9(2), 1-16.

https://doi.org/10.1145/1596543.1596548

\section{CSAC2 Human centered computing (ACM journal articles)}

Harper, F. M., \& Konstan, J. A. (2015). The movielens datasets: History and context. ACM Transactions on Interactive Intelligent Systems, 5(4), 1-19. https://doi.org/10.1145/2827872

Hiltz, S. R., Diaz, P., \& Mark, G. (2011). Introduction: Social media and collaborative systems for crisis management. ACM Transactions on Computer-Human Interaction, 18(4), 1-6. https://doi.org/10.1145/2063231.2063232

Lemos, A. L., Daniel, F., \& Benatallah, B. (2015). Web service composition: A survey of techniques and tools. ACM Computing Surveys, 48(3), 1-41. https://doi.org/10.1145/2831270 
Long, B., Seah, S. A., Carter, T., \& Subramanian, S. (2014). Rendering volumetric haptic shapes in mid-air using ultrasound. ACM Transactions on Graphics, 33(6), 1-10. https://doi.org/10.1145/2661229.2661257

Merrell, P., Schkufza, E., Li, Z., Agrawala, M., \& Koltun, V. (2011). Interactive furniture layout using interior design guidelines. ACM SIGGRAPH 2011 Papers on -

SIGGRAPH '11, 1. https://doi.org/10.1145/1964921.1964982

\section{CSAC2 Information systems (ACM journal articles)}

Guidotti, R., Monreale, A., Ruggieri, S., Turini, F., Giannotti, F., \& Pedreschi, D. (2018). A survey of methods for explaining black box models. ACM Computing Surveys, 51(5), 1-42. https://doi.org/10.1145/3236009

Lim, E.-P., Chen, H., \& Chen, G. (2013). Business intelligence and analytics: Research directions. ACM Transactions on Management Information Systems, 3(4), 1-10. https://doi.org/10.1145/2407740.2407741

Rodeh, O., Bacik, J., \& Mason, C. (2013). BTRFS: The Linux B-tree filesystem. ACM Transactions on Storage, 9(3), 1-32. https://doi.org/10.1145/2501620.2501623 


\section{CSAC2 Mathematics of computing (ACM journal articles)}

Bickel, B., Bächer, M., Otaduy, M. A., Lee, H. R., Pfister, H., Gross, M., \& Matusik, W. (2010). Design and fabrication of materials with desired deformation behavior. ACM Transactions on Graphics, 29(4), 1. https://doi.org/10.1145/1778765.1778800

Dow, S. P., Glassco, A., Kass, J., Schwarz, M., Schwartz, D. L., \& Klemmer, S. R. (2010). Parallel prototyping leads to better design results, more divergence, and increased self-efficacy. ACM Transactions on Computer-Human Interaction, 17(4), 1-24. https://doi.org/10.1145/1879831.1879836

Dunlavy, D. M., Kolda, T. G., \& Acar, E. (2011). Temporal link prediction using matrix and tensor factorizations. ACM Transactions on Knowledge Discovery from Data, 5(2), 1-27. https://doi.org/10.1145/1921632.1921636

Hildebrandt, K., Schulz, C., Tycowicz, C. V., \& Polthier, K. (2011). Interactive surface modeling using modal analysis. ACM Transactions on Graphics, 30(5), 1-11. https://doi.org/10.1145/2019627.2019638

Schumaker, R. P., \& Chen, H. (2009). Textual analysis of stock market prediction using breaking financial news: The AZFin text system. ACM Transactions on Information Systems, 27(2), 1-19. https://doi.org/10.1145/1462198.1462204 


\section{CSAC2 Networks (ACM journal articles)}

Gomez-Uribe, C. A., \& Hunt, N. (2015). The netflix recommender system: Algorithms, business value, and innovation. ACM Transactions on Management Information Systems, 6(4), 1-19. https://doi.org/10.1145/2843948

Krause, A., \& Guestrin, C. (2011). Submodularity and its applications in optimized information gathering. ACM Transactions on Intelligent Systems and Technology, 2(4), 1-20. https://doi.org/10.1145/1989734.1989736

Manshaei, M. H., Zhu, Q., Alpcan, T., Bacşar, T., \& Hubaux, J.-P. (2013). Game theory meets network security and privacy. ACM Computing Surveys, 45(3), 1-39. https://doi.org/10.1145/2480741.2480742

\section{CSAC2 Security and privacy (ACM journal articles)}

Alcantara, D. A., Sharf, A., Abbasinejad, F., Sengupta, S., Mitzenmacher, M., Owens, J. D., \& Amenta, N. (2009). Real-time parallel hashing on the GPU. ACM Transactions on Graphics, 28(5), 1. https://doi.org/10.1145/1618452.1618500 Pék, G., Butty’an, L., \& Bencsáth, B. (2013). A survey of security issues in hardware virtualization. ACM Computing Surveys, 45(3), 1-34. https://doi.org/10.1145/2480741.2480757

Shay, R., Cranor, L. F., Komanduri, S., Durity, A. L., Huh, P. (Seyoung), Mazurek, M. L., ... Christin, N. (2016). Designing password policies for strength and usability. 
ACM Transactions on Information and System Security, 18(4), 1-34.

https://doi.org/10.1145/2891411

\section{CSAC2 Software and it engineering (ACM journal articles)}

Hayes, G. R. (2011). The relationship of action research to human-computer interaction. ACM Transactions on Computer-Human Interaction, 18(3), 1-20. https://doi.org/10.1145/1993060.1993065

Peter, S., Li, J., Zhang, I., Ports, D. R. K., Woos, D., Krishnamurthy, A., ... Roscoe, T. (2015). Arrakis: The operating system is the control plane. ACM Transactions on Computer Systems, 33(4), 1-30. https://doi.org/10.1145/2812806

Shaer, O., \& Jacob, R. J. K. (2009). A specification paradigm for the design and implementation of tangible user interfaces. ACM Transactions on Computer-Human Interaction, 16(4), 1-39. https://doi.org/10.1145/1614390.1614395

\section{CSAC2 Theory of computation (ACM journal articles)}

Guo, K., Zou, D., \& Chen, X. (2015). 3d mesh labeling via deep convolutional neural networks. ACM Transactions on Graphics, 35(1), 1-12. https://doi.org/10.1145/2835487 
He, J., Zhang, Y., Huang, G., \& Cao, J. (2012). A smart web service based on the context of things. ACM Transactions on Internet Technology, 11(3), 1-23. https://doi.org/10.1145/2078316.2078321

Kazhdan, M., \& Hoppe, H. (2013). Screened poisson surface reconstruction. ACM Transactions on Graphics, 32(3), 1-13. https://doi.org/10.1145/2487228.2487237

Li, H., Weise, T., \& Pauly, M. (2010). Example-based facial rigging. ACM Transactions on Graphics, 29(4), 1. https://doi.org/10.1145/1778765.1778769

Rivers, A., Durand, F., \& Igarashi, T. (2010). 3D modeling with silhouettes. ACM Transactions on Graphics, 29(4), 1. https://doi.org/10.1145/1778765.1778846

Talton, J. O., Lou, Y., Lesser, S., Duke, J., Měch, R., \& Koltun, V. (2011). Metropolis procedural modeling. ACM Transactions on Graphics, 30(2), 1-14. https://doi.org/10.1145/1944846.1944851

\section{CSAC2 Algorithms (Textbooks)}

Kleinberg, J., \& Tardos, É. (2013). Algorithm design. Pearson.

\section{CSAC2 Theory of computation (Textbooks)}

Moore, C., \& Mertens, S. (2011). The nature of computation. OUP Oxford. 


\section{CSAC2 Data structures (Textbooks)}

Carrano, F. M., \& Henry, T. M. (2016). Data abstraction \& problem solving with $C^{++}$: Walls and mirrors. Pearson Education.

\section{CSAC2 Human centered computing (Textbooks)}

Reynolds, G. (2011). Ethics in information technology. Cengage Learning.

\section{CSAC2 Operating systems (Textbooks)}

Anderson, T., \& Dahlin, M. (2014). Operating systems: Principles and practice. Recursive Books.

\section{CSAC2 Mathematics of computing (Textbooks)}

Lehman, E., Leighton, F. T., \& Meyer, A. R. (2017). Mathematics for computer science. Samurai Media Limited. 


\section{CSAC2 Computer systems organization (Textbooks)}

Patterson, D. A., \& Hennessy, J. L. (2017). Computer organization and design RISC-V edition: The hardware software interface. Morgan Kaufmann.

\section{CSAC2 Probability and statistics (Textbooks)}

Devore, J. L. (2011). Probability and statistics for engineering and the sciences. Cengage Learning.

\section{CSAC2 Software design (Textbooks)}

Pilone, D., \& Miles, R. (2008). Head First software development. O’Reilly Media, Inc.

\section{CSAC2 Programming (Textbooks)}

Kernighan, B. W., \& Ritchie, D. M. (1988). The C programming language: 2nd edition. Prentice Hall. 